\title{
Experimental investigation of a sheared thermally unstable boundary layer
}

\author{
Dissertation \\ for the award of the degree \\ "Doctor rerum naturalium" (Dr.rer.nat.) \\ of the Georg-August-Universität Göttingen
}

within the doctoral program

Physics of biological and complex systems

of the Göttingen Graduate School of Neurosciences, Biophysics and Molecular

Biosciences (GGNB)

of the Georg-August University School of Science (GAUSS)

submitted by

Giuseppe Gabriele Nunnari

from Messina, Italy

Göttingen, 2021 


\section{THESIS COMMITTEE:}

Dr. Stephan Weiss (Referee)

Department of Fluid Physics, Pattern Formation and Biocomplexity,

Max Planck Institute for Dynamics and Self-Organization

Prof. Dr. Andreas Tilgner (Co-Referee)

Institute of Geophysics,

Georg-August-University Göttingen

Dr. Olga Shishkina

Department of Fluid Physics, Pattern Formation and Biocomplexity,

Max Planck Institute for Dynamics and Self-Organization

\section{MEMBER OF THE EXAMINATION BOARD:}

Dr. Stephan Weiss (Referee)

Department of Fluid Physics, Pattern Formation and Biocomplexity,

Max Planck Institute for Dynamics and Self-Organization

Prof. Dr. Andreas Tilgner (Co-Referee)

Institute of Geophysics,

Georg-August-University Göttingen

\section{OTHER MEMBERS OF THE EXAMINATION BOARD:}

Dr. Olga Shishkina

Department of Fluid Physics, Pattern Formation and Biocomplexity,

Max Planck Institute for Dynamics and Self-Organization

Prof. Dr. Jens Niemeyer

Institute for Astrophysics,

Georg-August-Universität Göttingen

Dr. Michael Wilczek

Theory of Turbulent Flows,

Max Planck Institute for Dynamics and Self-Organization

Prof. Dr. rer. nat. Dr. habil. Andreas Dillmann

Institute of Aerodynamics and Flow Technology,

German Aerospace Center (DLR) in Göttingen

Date of oral examination: 14/06/2021 


\section{Affidativ}

Hereby, I declare that the present thesis has been written independently and with no other sources and aids than quoted.

Göttingen June 28, 2021 
In memory of Dr. Antonio Anastasi (1989-2020). 


\section{Contents}

1 Introduction 1

2 Background $\quad 7$

2.1 Fundamental equations of fluid motion . . . . . . . . . . . . 7

2.2 Basic concepts in turbulence . . . . . . . . . . . . . . . . . . . 9

2.3 Velocity boundary layer without surface heating . . . . . . . . . . . . 11

2.3.1 Laminar boundary layer . . . . . . . . . . . . . . . . . . . 11

2.3.2 Turbulent boundary layer . . . . . . . . . . . . . . . . 14

2.3.3 The transition from a laminar to a turbulent boundary layer . . . . 20

2.4 Thermal boundary layer under shear forces . . . . . . . . . . . . . . . . 24

2.4.1 Laminar boundary layer . . . . . . . . . . . . . . . 25

2.4 .2 Turbulent boundary layer . . . . . . . . . . . . . . 27

2.4.3 The influence of heat on the velocity boundary layer . . . . . . . . 29

2.5 A note on dimensionless parameters and length scales . . . . . . . . . . . 31

3 Experimental setup and methods $\quad 35$

3.1 The Prandtl wind-tunnel . . . . . . . . . . . . . . . . . . 36

3.2 The heated plate . . . . . . . . . . . . . . . . . 40

3.2.1 Thermistors . . . . . . . . . . . . . . . . . . 42

3.2.2 Heated plate temperature feedback loop . . . . . . . . . . . . . . 47

3.2.3 Heated plate constant heat transfer . . . . . . . . . . . . . . 48

3.3 Constant Temperature Anemometry (CTA) . . . . . . . . . . . . . . . 49

3.3.1 Hot-wire probes . . . . . . . . . . . . . . . . . . . . 49

3.3.2 Height control system and probe holder . . . . . . . . . . . . . 52

3.4 Trip wire . . . . . . . . . . . . . . . . . . . 54 
3.5 Vertical thermistor bar . . . . . . . . . . . . . . . 55

3.6 High speed camera setup . . . . . . . . . . . . . . . 57

3.7 Experimental procedure . . . . . . . . . . . . . . . . 57

3.7.1 Heat transfer measurement . . . . . . . . . . . . . . . . . . . . 59

3.7.2 Temp controlled hot-wire velocity measurement . . . . . . . . . 59

3.7.3 Vertical thermistors measurements . . . . . . . . . . . 60

3.7.4 Camera Image collection . . . . . . . . . . . . . . . . . . . 61

4 Data analysis and results $\quad 63$

4.1 Velocity boundary layer without heating . . . . . . . . . . . 63

4.1 Turbulent velocity boundary layer . . . . . . . . . . . . . 63

4.1.2 Velocity boundary layer in transitional regime . . . . . . . . . . 73

4.1.3 Probing the transition from laminarity to turbulence using the MTERA method . . . . . . . . . . . . . . 80

4.2 Velocity boundary layer with heating . . . . . . . . . . . . . 85

4.2.1 Velocity boundary layer in turbulent regime with heated surface . . 85

4.2.2 Velocity boundary layer in the transitional regime . . . . . . . . . 101

4.2.3 Shear velocity correction and modeling . . . . . . . . . . . . 112

4.3 Heat transport measurements . . . . . . . . . . . . . . . . . . . . 118

4.3.1 Local heat transfer under constant shear stress . . . . . . . . . . . . 118

4.3.2 New length-scales and power-laws . . . . . . . . . . . . . . 125

4.4 Thermal boundary layer profile . . . . . . . . . . . . . . . . . 130 


\section{Symbols and Abbreviations}

$\alpha \quad$ Thermal diffusivity

$\beta \quad$ Thermal expansion coefficient

$\beta_{3} \quad$ Skewness

$\beta_{4} \quad$ Kurtosis

$\delta \quad$ Boundary layer height

$\epsilon \quad$ Boundary layer shift

$\eta \quad$ Boundary layer similarity variable

$\kappa \quad$ Thermal conductivity

$\mu \quad$ Dynamic viscosity

$\nu=\frac{\mu}{\rho}$ Kinematic viscosity

Re Reynolds number

$\operatorname{Re}_{x}=\frac{U_{0} x}{\nu}$ Boundary layer Reynolds number

$\operatorname{Re}_{\text {crit }}$ Reynolds number at which boundary layer becomes fully turbulent

$\operatorname{Re}_{\text {ind }}$ Critical Reynolds number at which the laminar Blasius profile becomes unstable

$\rho \quad$ Density

$\tau \quad$ Shear stress

$\tau_{w}=\tau(z=0)$ Wall shear stress 
$c_{f} \quad$ Skin friction coefficient

$c_{p} \quad$ Heat capacity

$G r_{x} \quad$ Boundary layer Grashof number

$k \quad$ Wave-number

L Typical length

$N u_{x} \quad$ Boundary layer Nusselt number

$p \quad$ Pressure

$\operatorname{Pr}=\frac{\nu}{\alpha}$ Prandtl number

$q_{w} \quad$ Wall heat flux

Ri Richardson number

T Temperature

$t \quad$ Time

$T_{0} \quad$ Free flow Temperature

$u \quad$ Velocity component along flow direction

$u^{\prime} \quad$ Velocity fluctuations

$u^{+}=\frac{u}{u_{\tau}}$ Dimensionless velocity

$U_{0} \quad$ Free flow velocity

$u_{\text {std }} \quad$ Velocity standard deviation

$v \quad$ Vertical velocity component

$x \quad$ Distance from the leading edge of the experimental surface

$z \quad$ Vertical distance from the surface

$z^{+}=\frac{z u_{\tau}}{\nu}$ Dimensionless vertical position 


\section{Chapter 1}

\section{Introduction}

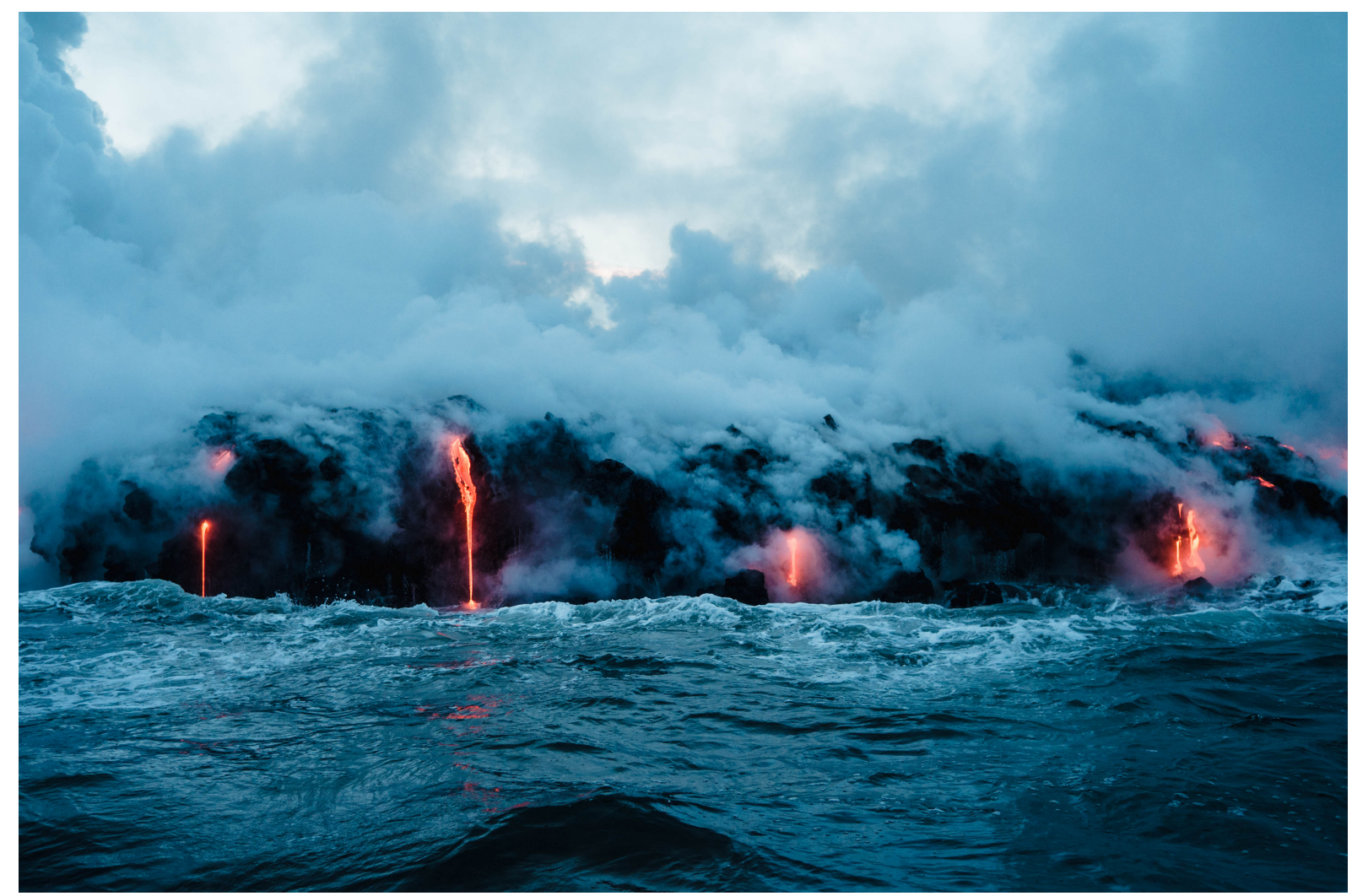

Figure 1.1: Volcanic lava flows into the ocean generating vapor clouds that are transported away by the wind - Creative Common license photo

In the first picture, we can admire lava flowing into the ocean exchanging extremely fast a huge amount of heat and solidify. In this beautiful picture, we are looking at multiple different heat transfer mechanism in action. The red glowing color of the lava is the consequence of heat loss by radiation. After the lava has solidified, heat is transported inside the volcanic rock purely by conduction along temperature gradients. The hot lave 
that reaches the water heats it above its boiling point so that heat is converted into enthalpy of vaporization. The resulting hot steam is clearly visible as the smoke that rises by free convection due to its reduced density compared to the colder surrounding air. The warm vapour is then carried away from the wind that blows inland and that transports the heat by forced convection. All these phenomena occur at the same time and interact with each other.

The understanding of the interaction between the different heat transfer mechanisms has been proven to be an extremely challenging goal. Our study attempts to improve our knowledge in this field, with a focus on free, forced and mixed convection. Thermal convection is one of the most important heat transfer mechanisms. It is driven by density gradients induced in a fluid for example by temperature gradients. The higher temperature in general results in lower fluid density. The density gradient generates a force described by Archimedes's principle $F_{b}=-\rho g V$, with the lighter fluid elements pushed against the gravity normal. This phenomenon is named free or natural convection.

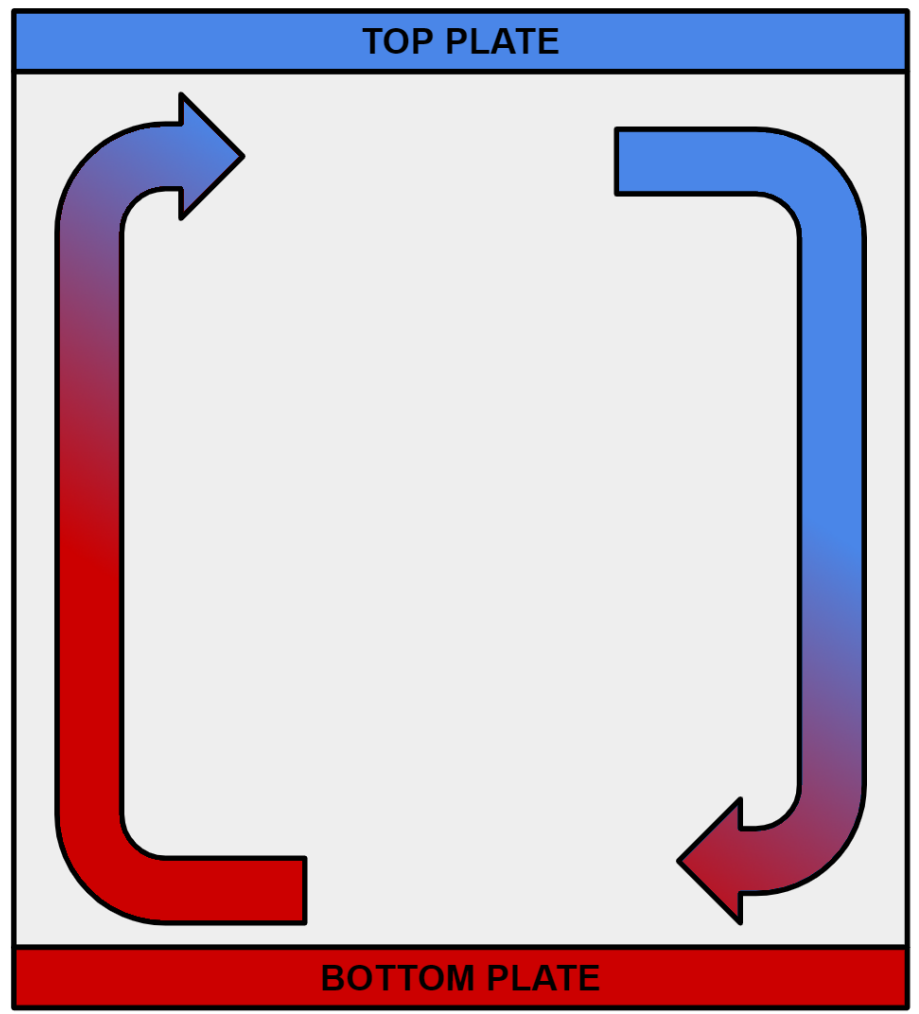

Figure 1.2: Natural convection cycle

Natural convection is mainly investigated by the Rayleigh-Bénard Convection community using the "Rayleigh-Bénard convection cells". There, a horizontal fluid layer is 
heated from below and cooled from above. The warm fluid of lower density rises towards the top, cools down and sinks due to its increased density back to the bottom. In laterally confined systems, as sketched in fig. 1.2, the resulting fluid motion has the form of a large convection role where the warm fluid rises at one side and the cold fluid sinks at the opposite side. Convection is one of the most important heat transfer mechanisms in nature. It is essential for life on our planet, playing a crucial role in the water cycle, as much as in other fields as i.e.: meteorology, oceanography, geology, industrial applications, and more. It is fundamental in those study to rely on robust models that allow us a better understanding of those mechanisms.

We cannot separate all the heat transfer mechanisms acting on a system in many of the applications. In general, heat can also be passively advected by the flow while buoyancy plays only a negligible role in creating the flow. Then, the heat transfer depends on the different mechanism that drives the flow. For example, when the surface of a hot solid is exposed to a cold flow, the heat transfer strongly depends on the velocity component normal to the surface. This velocity component is often created by shear stresses due to gradients of the velocity parallel to the surface, which render the flow close to the boundary turbulent. In this case, one can look at active advection (convection) and passive advection as two faces of the same coin, with the advection directly correlated with the sheer force and the convection with the buoyant force. It is extremely common to have both of these mechanisms in nature acting on the same system and it is crucial to improve our understanding of the interaction between these two mechanisms and how they affect the total heat transfer.

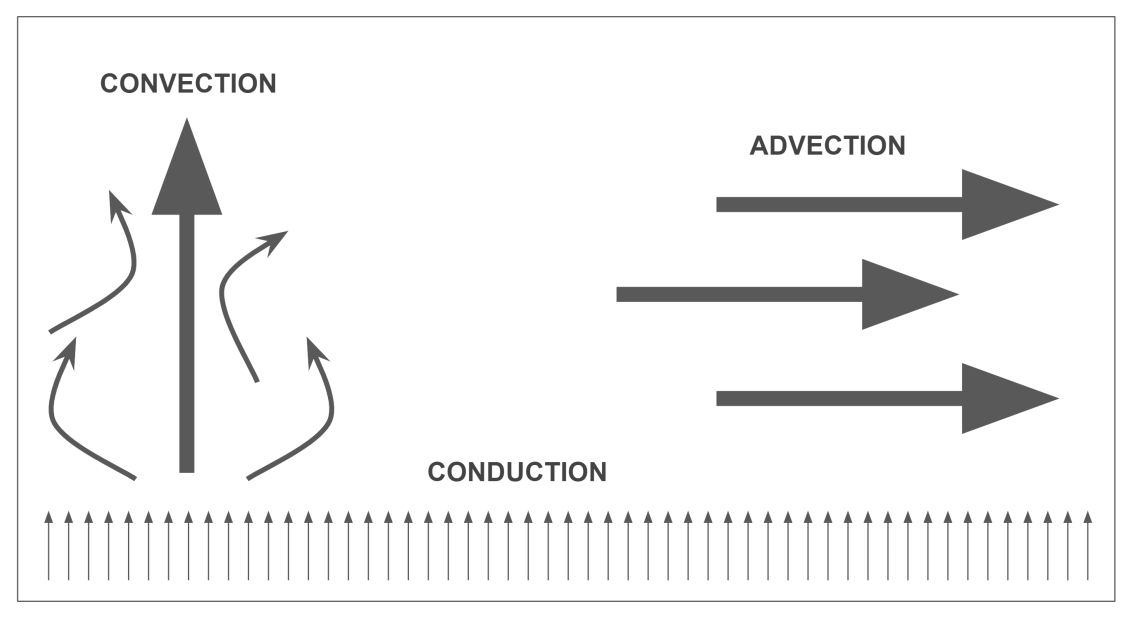

Figure 1.3: Main heat exchange processes 
The flow is considered to exhibit forced convection when the shear force is dominant over the buoyant force. We consider it to be mixed convection when the shear force is comparable with the buoyant force. We consider it in free convection or natural convection, when the shear force is dominated by the buoyant force.

As in our example above, very often one is interested in the heat transfer across a phase boundary between a solid/liquid, liquid/gas, or gas/liquid. A good example can be the ocean surface that absorbs the radiation from the sun, heats up and then exchange the heat with the atmosphere. When a fluid moves along a surface, viscosity creates a slower layer of fluid close to the surface that is called boundary Layer.

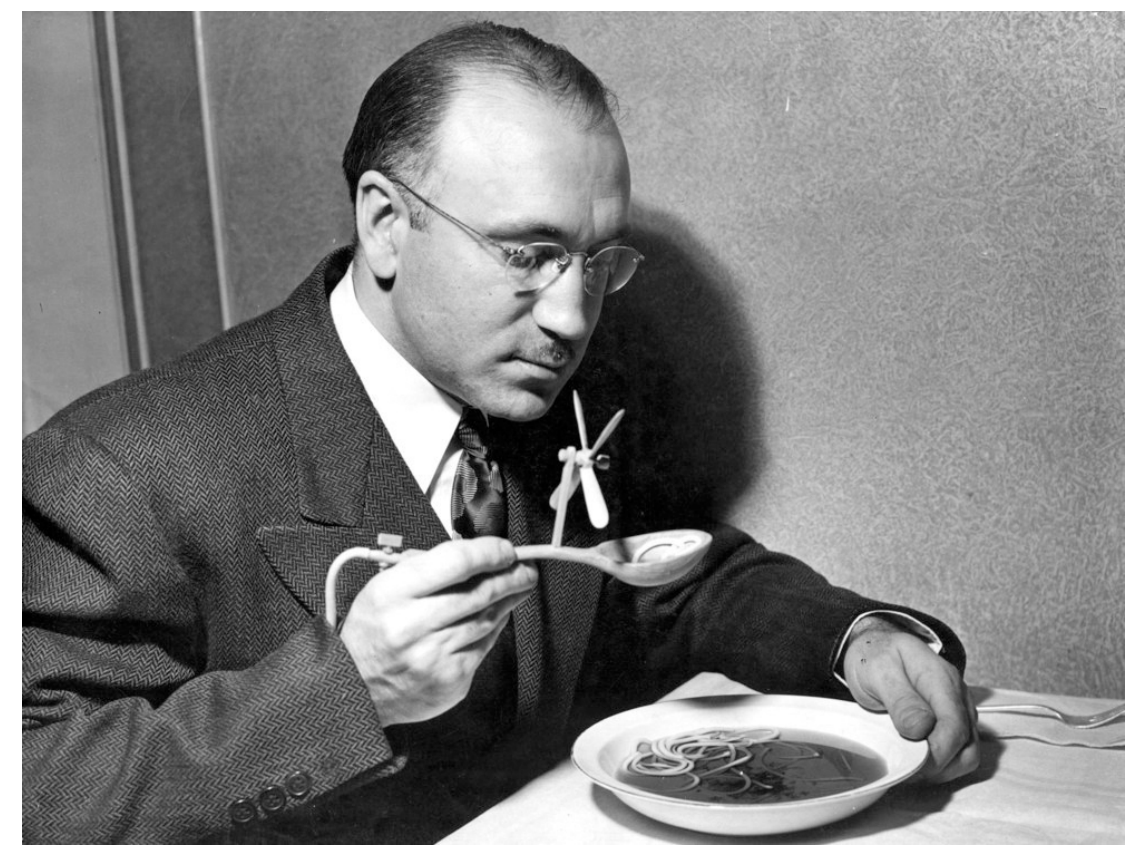

Figure 1.4: Forced convection is applied to increase the heat transfer at the spoon surface. Image credit: Hans Reinhart and Stringer, collection Hulton Archives 1948

At the wall proximity, where the boundary layer develops, the flow moves slowly and develop a velocity field that is a function of the distance from the surface and the viscosity of the fluid. In the pure shear flow, the boundary layer is considered to have 3 main regimes, the laminar regime, the turbulent regime, and the transitional regime ${ }^{1}$.

The evolution of the velocity field in a pure shear flow has been studied and modelled for more than 100 years. Despite the extensive study of the boundary layer evolution, there is a lack of models and experiments that can describe it when in a mixing convection regime. It is of crucial importance to collect data in those regimes to improve our

\footnotetext{
${ }^{1}$ More details in Chapter 2
} 
understanding of the dynamics ongoing in this multidisciplinary context. Some of the most fundamental questions that are still unanswered are:

- How does thermal convection influences the boundary layer development in different convectional regimes and different boundary layer regimes?

- How does the boundary layer regime affect the heat transfer under buoyant forces?

- What is the evolution of the fluid properties during the transition between natural and forced convection?

- Can we find a simple method that allows us to predict the heat transfer when shear and buoyant forces are known and vice-versa?

In the urge to provide new empirical data to answer those questions, we have designed and built a new experiment in which details and results will be presented in this work. Our experimental setup allows us to control the free flow velocity and surface temperature. This allows us to produce data in both transitional and turbulent boundary layer regime, but also spans between natural, mixed and forced convection. We hope with our work to offer our help in the understanding, and advance in this field of study.

We believe that our results could be considered beneficial for the fields of study such as Atmospheric boundary layer, Mixed convection, CFD flow simulations model for buoyant fluids, Industrial applications, Oceanography, Geology, and Thermal convection. 


\section{Chapter 2}

\section{Background}

\subsection{Fundamental equations of fluid motion}

Our study will focus on fluids that are: Newtonian, isotropic, and incompressible. Let us start by considering the conservation laws for momentum and mass. We consider a flow with pressure $p(\mathbf{x}, t)$, velocity field $\mathbf{u}(\mathbf{x}, t)$, and mass density $\rho(\mathbf{x}, t)$, with $\mathbf{x}$ being the position in space and $t$ denoting time. The conservation of mass can be written as:

$$
\frac{\partial \rho}{\partial t}+\nabla \cdot(\rho \mathbf{u})=0
$$

The mathematical description of fluid flows can be significantly simplified when the flow can be considered incompressible. This is the case when the density of a fluid parcels remains constant along its trajectory. This assumption is valid as long as the flow velocity is small compared to the speed of sound $\left(\sqrt{\frac{d p}{d \rho}}\right)$. For incompressible fluids, we can write eq. 2.1 as:

$$
\nabla \cdot \mathbf{u}=0
$$

The total change of the momentum of a fluid element, is given by the surface and volume forces acting on it. We can then write:

$$
\rho\left(\frac{\partial}{\partial t}+\mathbf{u}(\mathbf{x}, t) \cdot \nabla\right) \mathbf{u}(\mathbf{x}, t)=\rho \boldsymbol{f}(\mathbf{x}, t)+\nabla \cdot \boldsymbol{\sigma}(\mathbf{x}, t)
$$

with $\boldsymbol{f}(\mathrm{x}, t)$ being the volume force acting on the fluid element, and $\boldsymbol{\sigma}(\mathrm{x}, t)$ being the stress tensor that describes the forces acting on the surface of the fluid element. For a 
Newtonian fluid with a constant dynamic viscosity $\mu$, the divergence of the stress tensor is written as:

$$
\nabla \cdot \boldsymbol{\sigma}(\mathbf{x}, t)=-\nabla p(\mathbf{x}, t)+\mu \Delta \mathbf{u}(\mathbf{x}, t)
$$

We can now plug eq. 2.4 in the eq. 2.3, divide by $\rho$, and obtain the Navier-Stokes equations,

$$
\left(\frac{\partial}{\partial t}+\mathbf{u}(\mathbf{x}, t) \cdot \nabla\right) \mathbf{u}(\mathbf{x}, t)=-\frac{1}{\rho} \nabla p(\mathbf{x}, t)+\nu \Delta \mathbf{u}(\mathbf{x}, t)+f(\mathbf{x}, t)
$$

with $\nu=\mu / \rho$ denoting the kinematic viscosity.

It is often helpful to make this equation (2.5) dimensionless. For this, we re-scale $\mathbf{u}$, $t, \mathbf{x}, f, p$ and the differential operators in eq. 2.5 with appropriate scales for the length $L$, velocity $U$, time $L / U$, and pressure $\rho U^{2}$ and obtain :

$$
\left(\frac{\partial}{\partial t}+\mathbf{u}(\mathbf{x}, t) \cdot \nabla\right) \mathbf{u}(\mathbf{x}, t)=-\nabla p(\mathbf{x}, t)+\frac{1}{R e} \Delta \mathbf{u}(\mathbf{x}, t)
$$

with Reynolds number $R e=\frac{\mathrm{u} L}{\nu}$ that represents the ratio of the inertial forces over the viscous forces and that is used as a reference for the strength of the turbulence in a given flow.

In flows with a low value of $R e$, the viscous forces dominate over the inertial forces. The viscous forces keep the flow smooth and damp the fluctuations preventing the fluid layers to mix. In a laminar flow, there is no exchange of mass between the planes perpendicular to the flow direction and the velocity fluctuations are zero.

With the increase of $R e$, the influence of inertia becomes stronger and the flow becomes unsteady, chaotic and finally turbulent. For a flow above a flat surface, Burgers in 1924 [1], Van der Hegge Zijnen again in 1924 [2], Hansen in 1928 [3], and Dryden in 1939 [4] have experimentally found a critical Reynolds number $\operatorname{Re}_{\text {crit }} \approx 5 \times 10^{5}$ at which the laminar steady flow becomes unsteady. The exact value of $\operatorname{Re}_{\text {crit }}$ may change due to external parameters such as the outer flow turbulence level and surface smoothness, between $3 \times 10^{5}<\operatorname{Re}_{\text {crit }}<2 \times 10^{6}$. The turbulent flow is usually referred to as chaotic, this is because of the stochastic nature of its fluctuations. In a turbulent flow, there is a strong exchange of mass, momentum and other transported quantities, in the planes perpendicular to the flow direction, by eddies and strong velocity fluctuations. 
The Reynolds number allows us to compare flows in systems of different scales but similar geometry. Note that the Reynolds number compares inertia and viscous forces on the length and velocity scales included in it. On sufficiently small length scales, for example, the Reynolds number is always small and the flow laminar. The length scale at which Re becomes close to one is the Kolmogorov length scale.

\subsection{Basic concepts in turbulence}

Chaotic flows have been described already by Leonardo Da Vinci more than 500 years ago, and he named such fluid motion "turbolenze", a term that is still in use in the Italian language to describe turbulence. It took more than 400 years to begin to understand turbulence and to develop a rigorous mathematical treatment of it, with fundamental contributions from i.e. Reynolds [5], Taylor [6], and Kolmogorov [7]. Taylor in 1935-36 has published a series of papers [8-12] where he presents turbulence as a random phenomenon and introduces then a series of statistical tools that help in the analysis of homogeneous and isotropic turbulence. In 1941 a paper published in Russian from Kolmogorov [7] has provided one of the most important statistical theories for turbulence. In this paper Kolmogorov provides a theoretical framework based on scaling analysis to describe the isotropic and homogeneous turbulent flow. Here, isotropic means that there is no mean flow $^{1}$ and that two-point statistical quantities, like correlation or structure functions, only depend on the distance between the two points, but not on the direction of their difference vector. Effects like buoyancy and rotation cause a flow to be anisotropic. Homogeneous means that any statistical quantity (average or RMS values) is the same for any point in space.

It is important to keep in mind that isotropy, and homogeneity are approximations and we need to evaluate every time if we can apply these assumptions or not. A major prediction of Kolmogorov theory is the turbulent energy cascade. The analysis of Energy density spectra does allow us to study the turbulent kinetic energy distribution along with different length scales in a turbulent flow. When we refer to the Kolmogorov scaling law [7], we point at the predicted $k^{-5 / 3}$ energy scaling that characterizes the inertial subrange region (defined below).

\footnotetext{
${ }^{1}$ In case of a mean flow it can be subtracted from the instantaneous velocity field.
} 
According to the turbulent model of Richardson, [13], kinetic energy is introduced into the flow at the larges scales, which are in fact the largest eddies that gather their energy from the main flow. The size of those large eddies is determined by the flow velocity and the geometry of the system. This length scale is called Energy injection range or Production range. It is characterized by a peak in the energy spectrum $E(k)$, with $k$ being a typical wave number, i.e., the inverse of the typical length scale.

The eddies of a certain size break into smaller eddies and in this way transfer kinetic energy from large to small scales. There has to be a range at which those eddies become so small that the viscosity is not negligible and therefore the kinetic energy of these eddies is dissipated as heat. The length scale where this happens is referred to as Viscous dissipation range. This range is characterized by an extremely fast decay of the energy with wave number and of course, it limits the size of the smallest eddies size that can be found in a flow (fig. 2.1).

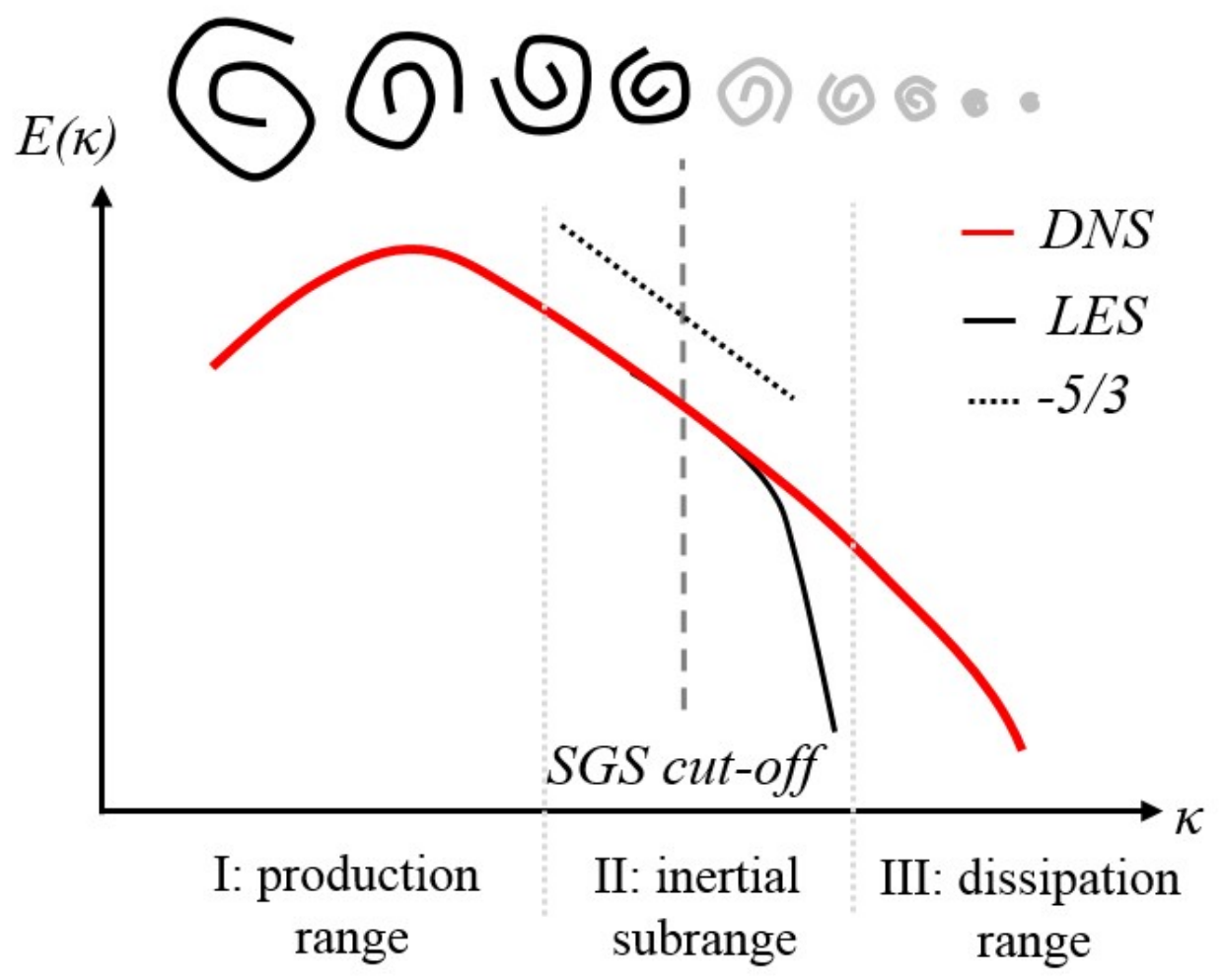

Figure 2.1: Typical energy spectrum in a turbulent flow, and a schematic of the energy cascade. Ref. [14]

What Kolmogorov has presented in 1941 [7] is a model for the transfer of the energy to subsequently smaller eddies in a range where the viscous dissipation into heat is negligible. 
This range is usually referred to as inertial subrange. In this range, one can derive from simple scaling arguments assuming isotropy and homogeneity, how the 2nd order structure function $\left\langle\left[\Delta u_{x}^{\prime}\right]^{2}\right\rangle$ depends on the distance between two points $r$ and the dissipation rate of the turbulent kinetic energy $\varepsilon$ :

$$
\left\langle\left[\Delta u_{x}^{\prime}\right]^{2}\right\rangle \sim \varepsilon^{\frac{2}{3}} r^{\frac{2}{3}}
$$

Onsager in 1945 [15] applied the same idea and obtained for the spectral distribution of turbulent kinetic energy in the inertial subrange

$$
E(k)=\alpha \varepsilon^{\frac{2}{3}} k^{-\frac{5}{3}},
$$

with $\alpha$ being the Kolmogorov constant. Both power laws are expected to be valid for length scales that are significantly larger than the Kolmogorov scale $\eta=\left(\nu^{3} / \varepsilon\right)^{1 / 4}$ and significantly smaller than the integral length scale that correspond to the size of the largest eddies.

\subsection{Velocity boundary layer without surface heating}

\subsubsection{Laminar boundary layer}

Let us consider a low viscosity $\nu$ fluid that moves along a fixed surface with a relative velocity $U_{0}$. At the interface between solid and fluid the adhesive forces are predominant and therefore the fluid cannot move with respect to the surface. The fluid velocity at the solid surface has to be $u_{(z=0)}=0$. This is called the no-slip condition. Under the hypothesis of no viscous interaction, and therefore no connection between the layers of fluid, we would not have a continuous transition of velocity in between the $u_{(z=0)}=0$ at the wall surface and $u_{(z=\infty)}=U_{0}$.

In order to characterise theoretically the influence of boundaries on the flow, L. Prandtl in 1904 [16] has suggested dividing the flow into two regions. One region is far from the surface, where the effect of the viscosity becomes negligible and therefore one can consider this region inviscid. This region is commonly referred as outer flow or bulk.

The other region is close to the surface, where viscosity effects cannot be ignored. In 
this region, a velocity gradient develops perpendicular to the solid boundary. This region is usually referred to as boundary layer. A sketch of such a boundary layer is shown in fig. 2.2 .

Let us assume a solid surface at $\mathrm{z}=0$ that extends in the $\mathrm{x}$-direction and a flow parallel to it with a bulk velocity $U_{0}$ far away from the surface. Figure 2.2 shows the development of a boundary layer and the velocity profile as they evolve beginning from the leading edge of the plate at $\mathrm{x}=0$. With increasing distance from the leading edge, the height of the boundary layer grows. In order to quantify its size, we introduce the concept of boundary layer limit $\delta_{99}(x)$, defined as the distance from the plate at which the time-average velocity has reached $0.99 \cdot U_{0}$.

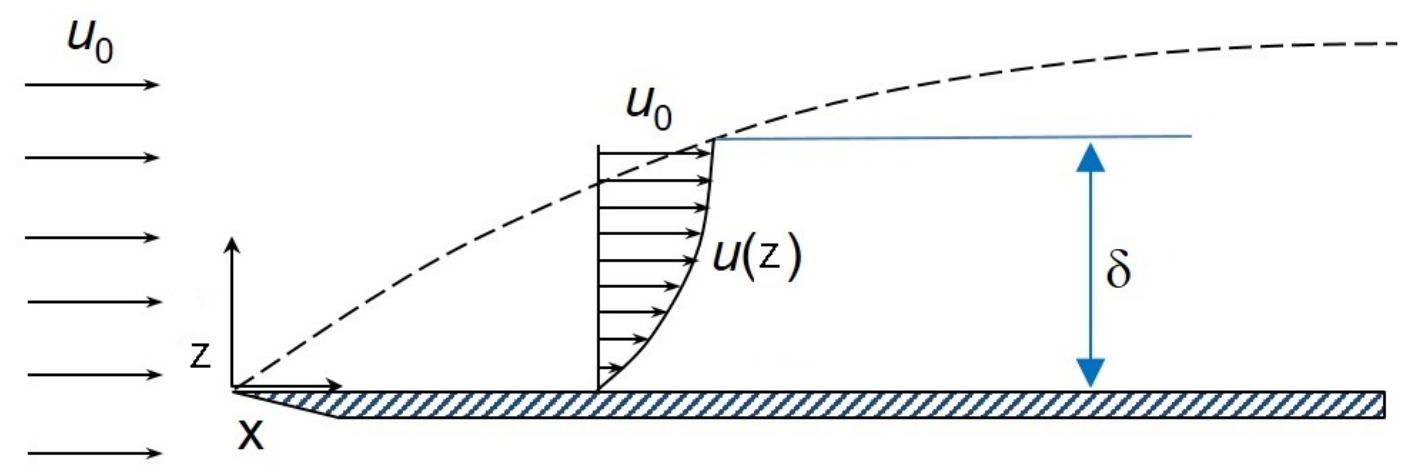

Figure 2.2: Development of a boundary layer over a flat surface.

Assuming that the flow close to the surface is laminar, then the momentum transport in due to viscous diffusion and we can estimate the boundary layer thickness $\delta(x)$ by balancing the inertial forces with the viscous forces:

$$
\mu \frac{U_{0}}{\delta^{2}} \sim \frac{\varrho U_{0}^{2}}{x}
$$

which we can solve for $\delta(x)$ to:

$$
\delta \sim \sqrt{\frac{\nu x}{U_{0}}}=\frac{x}{\sqrt{\operatorname{Re}_{x}}}
$$

with the appropriate Reynolds number $\operatorname{Re}_{x}=U_{0} x / \nu$, and $x$ being distance from the leading edge of the flat surface.

It was H. Blasius (1908) [17] then who determined the exact solution for a stationary flow over a flat plate with zero angles of incidence in dimensionless form. By defining a 
similarity variable for the wall-normal direction $\eta=z \sqrt{x \nu / U_{0}}$, Blasius could derive an analytical equation for a re-scaled stream function $f(\eta)=\psi / \sqrt{2 \nu x U_{0}}$ to be $^{2}$ :

$$
f^{\prime \prime \prime}+f f^{\prime \prime}=0
$$

By solving this equation also the relevant coefficient for eq. 2.10 can be determined to

$$
\delta_{99}(x)=5 \frac{x}{\sqrt{R e_{x}}}
$$

Another relevant parameter, which represents the momentum transport from the solid wall in wall-normal direction is the wall shear stress $\tau_{w}$, also known as skin friction drag. For a Newtonian fluid $\tau_{w}$ is proportional to the dynamic viscosity and the velocity gradient at the wall:

$$
\tau_{w}=\mu\left(\frac{\partial u}{\partial y}\right)_{y=0} .
$$

Since momentum is predominantly transported inside the boundary layer by viscous diffusion, the wall shear stress can be estimated for laminar boundary layers by using the proportion $\partial u / \partial y \sim U_{0} / \delta$ to:

$$
\tau_{w} \sim \mu \frac{U_{0}}{\delta}
$$

The wall shear stress is often normalised by the dynamic pressure $\rho U_{0}^{2} / 2$ resulting in the dimensionless skin friction coefficient:

$$
c_{f}=\frac{\tau_{w}}{\frac{1}{2} \rho U_{0}^{2}}
$$

In research one is now interested how $c_{f}$ depends in a given geometry on the control parameter, such as $\mathrm{Re}_{x}$. For a laminar boundary layer this can easily be calculated from the Blasius solution and results in:

$$
c_{f}=\frac{0.664}{\sqrt{R e_{x}}} .
$$

\footnotetext{
${ }^{2}$ Here, $\psi(x, y)$ is the stream function so that $u_{x}=\frac{\partial \psi}{\partial y}$ and $u_{y}=-\frac{\partial \psi}{\partial x}$.
} 


\subsubsection{Turbulent boundary layer}

A laminar boundary layer becomes unstable and turns turbulent when the Reynolds number exceeds a critical value $\operatorname{Re}_{x}>\mathrm{Re}_{\text {crit }}$. Then, inertia exceeds the damping viscous forces and small perturbations of the laminar (Blasius) profile grow as they are carried along with the mean flow in a streamwise direction. One usually considers the boundary layer fully turbulent when the flow is highly fluctuating without intermittent laminar patches. The most notable change when the boundary layer has turned turbulent is the increase in vertical momentum transport, i.e., the wall shear stress $\tau_{w}$, and subsequently an increase of the boundary layer thickness. This is because momentum is now transported predominantly by advection which is a much more efficient transport mechanism compared to the diffusive transport for the laminar case.

While most momentum in a turbulent boundary layer is transported by advection, the velocities close to the surface are rather small and have to vanish right at the surface. Hence, in the very proximity of the wall, i.e., inside the inner layer, inertia is small and viscous diffusion is the main mechanism for the momentum transport.

Von Karman in 1930 [18] has set the basis for the universal law of the wall for a flat surface. In his study, he proposed a model that describes the functional relation between the skin friction $c_{f}$ and the Reynolds number Re. His suggestions are in accordance with the ideas of Prandtl and Nikuradse, who suggested functional relations between the skin friction and the Reynolds number for pipe and channel flows [19]. These concepts have been carried on by Izakson [20], Millikan [21], and Mises [22], who considered a turbulent boundary layer on top of a flat surface with zero inclination. For this situation, the most inner part of the boundary layer is only coupled to the outer parts via the momentum transport $\tau_{w}$ and hence $\tau_{w}$ must determine the velocity profile $U(z)$ of its inner part. Therefore, one defines velocity and length scales that are used for normalisation based on $\tau_{w}$. These are the shear or skin friction velocity

$$
u_{\tau}=\sqrt{\frac{\tau_{w}}{\rho}}
$$

and the corresponding length scale $\nu / u_{\tau}$. From dimensional arguments we can see that 
the mean velocity profile close to the wall $z \ll \delta$ must satisfy the Prandtl law of the wall:

$$
U(z)=u_{\tau} f\left(\frac{u_{\tau} z}{\nu}\right)
$$

On the other hand, if Re is high enough the boundary layer size $\delta$ is much larger than the viscous sublayer and the von Karman velocity defect law has to be valid in the outer region of the boundary layer, i.e., for $z^{+}=z u_{\tau} / \nu \gg 1$ :

$$
U_{0}-U(z)=u_{\tau} f_{1}\left(\frac{z}{\delta}\right)
$$

If one furthermore assumes that

$$
U_{0}=u_{\tau} f_{2}\left(\frac{u_{\tau} \delta}{\nu}\right)
$$

one can solve for functions $f\left(u_{\tau} z / \nu\right), f_{1}(z / \delta)$ and $f_{2}\left(\delta u_{\tau} / \nu\right)$. In particular, we obtain (see Izakson [20]) for $z$ sufficiently far away from both the surface and the upper end of the boundary layer an expression for the normalised velocity $u^{+}:=u / u_{\tau}$ as a function of the normalised distance from the plate $z^{+}=z u_{\tau} / \nu$ :

$$
u^{+}\left(z^{+}\right)=\frac{1}{k} \ln z^{+}+B
$$

The coefficient $k$ is called the von Karman constant and it has been determined from measurements to be $k=0.41$. The offset $B$ has been measured with less precision to be $B \approx 5$. The value of $B$ depends on the flow characteristics and the geometry. Furthermore, with the consideration above, one can write an implicit relation for the skin friction coefficient in a turbulent boundary layer:

$$
\sqrt{2 / c_{f}}=\frac{1}{k} \ln \left(\operatorname{Re}_{x} c_{f}\right)+C
$$

Measurements for boundary layers over a flat plate resulted in $C \approx 2.4$ [23]. An explicit expression that approximate $c_{f}$ fairly well was proposed by White in 1969 [24] as

$$
c_{f}=\frac{0.451}{\left[\ln \left(0.056 \cdot \operatorname{Re}_{x}\right)\right]^{2}} .
$$


A typical evolution of $c_{f}$ as a function of $\operatorname{Re}_{x}$ is shown in fig. 2.3. For small $\operatorname{Re}_{x}$, the flow is laminar and $c_{f}\left(\operatorname{Re}_{x}\right)$ follows eq. 2.16 (red line). For very large $\mathrm{Re}_{x}$, the boundary layer is turbulent and $c_{f}\left(\operatorname{Re}_{x}\right)$ follows the implicit relation given in eq. 2.21. At a critical $\operatorname{Re}_{x}$ the BL bifurcates away from the laminar branch and converges asymptotically to the turbulent branch. Note that the exact point where $\operatorname{Re}_{x}$ becomes critical highly depends on the experimental condition, such as the smoothness of the plate surface, pressure gradients or an unsteady bulk flow.

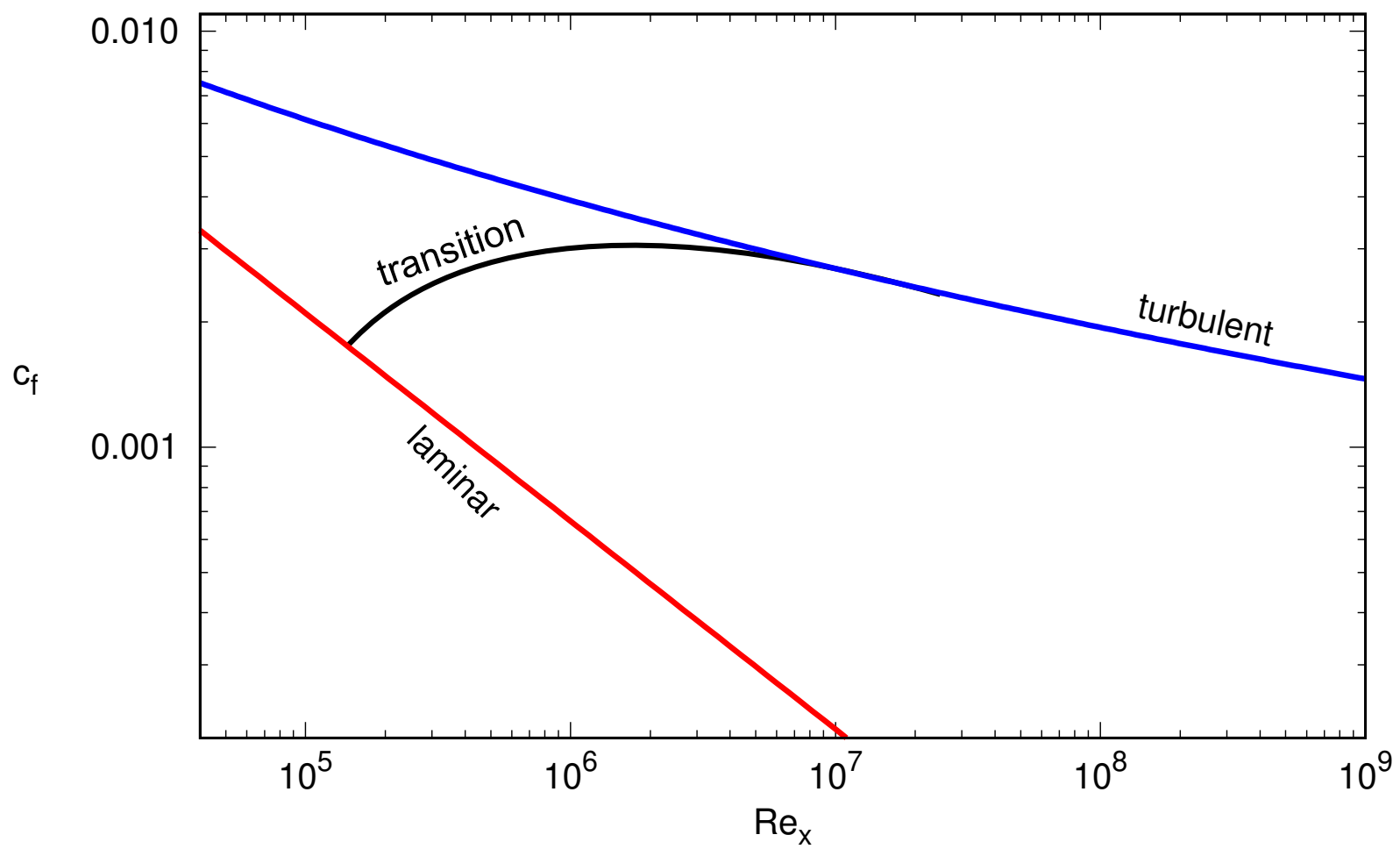

Figure 2.3: Skin friction coefficient $c_{f}$ as function of the shear force Re $e_{x}$ for a flow over a flat plate with zero incidence. The red line (laminar) and blue line (turbulent) represent eq. 2.16 and eq. 2.21. The black line is a drawn by hand and should represent $c_{f}$ for a transitional boundary layer assuming a critical Reynolds number $R e_{x} \approx 10^{5}$.

The average velocity profile of the inner region of any fully turbulent boundary layer is always identical when expressed in units of $u^{+}$and $z^{+}$as shown in fig. 2.4. In particular, one can distinguish three regions, namely

- for $0 \leq z^{+}<5$, the viscous sub-layer, where viscosity plays a dominating role and where $u^{+}=z^{+}$,

- for $5<z^{+}<70$, a transition or buffer layer, where momentum is transported both by viscous and advective diffusion 
- for $70<z^{+}$the logarithmic layer, where momentum is transported predominantly by advection and where $u^{+}=\frac{1}{k} \ln \left(z^{+}\right)+B$.

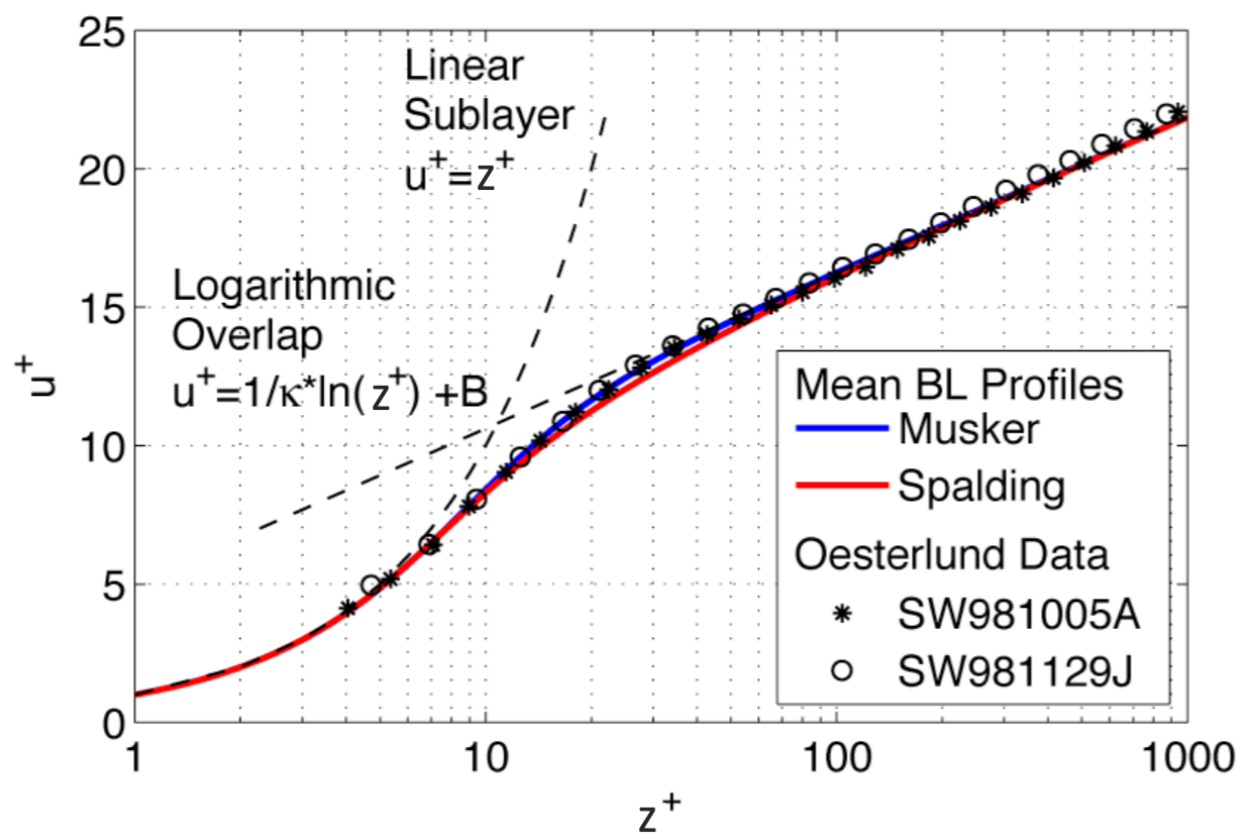

Figure 2.4: Comparison of Musker and Spalding profiles $(\kappa=0.41, B=5)$ with Österlund dataset from [25]. Figure from Kendall 2006 [26]

In order to scale the real measurement data in dimensionless wall units $u^{+}$and $z^{+}$it is necessary to determine the skin friction velocity $u_{\tau}$. There are different methods for this. The most straightforward way to do this is by measuring directly the velocity gradient at the wall, i.e., $\left.\frac{\partial u}{\partial z}\right|_{z=0}$. However, this is only possible for sufficiently larger boundary layers or small Re, because the viscous sub-layer is in many turbulent boundary layers too small to be sufficiently resolved with common measurement techniques. Instead, one often fits eq. 2.20 to the data, assuming constant parameters $k$ and $B$ and finding $u_{\tau}$ as a fit parameter [27]. This approach works particularly well for flows of very large Re with a log-region that extends over many orders of magnitudes.

An alternative method that works better for small Re, was suggested by A.J. Musker [28]. He suggested to approximate the average velocity in the viscous sub-layer, the buffer layer and the log-layer based on a model for the eddy viscosity as:

$$
\frac{d u^{+}}{d z^{+}}=\frac{\frac{\left(z^{+}\right)^{2}}{\kappa}+\frac{1}{s}}{\left(z^{+}\right)^{3}+\frac{\left(z^{+}\right)^{2}}{\kappa}+\frac{1}{s}}
$$


Here, $\kappa$ is again the von Karman constant and $s$ is a parameter that was estimated to $s=1.093 \cdot 10^{-3}[26]$ by fitting eq. 2.23 to data from the Österlund database [25].

We note that a similar attempt for modelling the lower part of the turbulent boundary layer was made by Spalding in 1961 [27] (red curve in fig. 2.4). In this thesis, we will use the approach by Musker. For a quantitative comparison of both models, we refer to the paper by Kendall and Koochesfahani 2006 [26]. Both methods are meant to fit the profile with the "Universal Law of the Wall", therefore it can be applied only for fully turbulent boundary layers.

While the inner part of a turbulent boundary layer scales with the skin friction velocity $u_{\tau}$, the velocity deficit in the outer part scales with the boundary layer height $\delta$. While for a laminar boundary layer, $\delta\left(\mathrm{Re}_{x}\right)$ can be calculated precisely, for turbulent boundary layers there is no simple expression. One reason is that one needs to take into account at which point $x_{c}$ the boundary layer actually becomes turbulent. In good approximation, the boundary layer height can expressed as (see [29]):

$$
\delta(x)=0.37 \frac{x}{R e_{x}^{1 / 5}}
$$

While a better approximation for $\delta(x)$ has been developed by Gersten and Herwig [30], eq. 2.24 is a sufficiently good approximation for the $\mathrm{Re}_{x}$ range we are investigating in our experiment and hence we will use it to estimate our $\delta$.

Energy spectra in a turbulent boundary layer. Above in sec. 2.2 we have briefly discussed the 2nd order structure function as well as the spectral distribution of kinetic energy for isotropic turbulence. We have mentioned Kolmogorov's famous 2/3-law and the 5/3-law. An important question is, whether these laws also hold in turbulent boundary layer which is, in fact, neither very homogeneous nor isotropic. The answer depends on the length scale $r$ and the distance $z$ from the wall. In particular, in the outer part of the boundary layer, the Kolmogorov $2 / 3$ law (eq. 2.7) still holds as long as $r$ is sufficiently smaller than $\delta$. This is because the large eddy has to break down to sufficiently small scales until the information about the orientation of the largest eddies is lost and isotropy is restored. And of course, the 2/3-law only holds for the inertia range and hence in the range $\eta \ll r \ll \delta$, with $\eta$ being the Kolmogorov micro-scale.

The $l$-range where isotropic turbulence can be assumed to decrease with decreasing 
distance to the wall. Close to the wall, the flow must be anisotropic over all length scales involved because the vertical fluctuations decrease much faster than the horizontal fluctuations. Perry, Henbest, and Chong in 1986 [31], have suggested a new regime that appears between the $r^{2 / 3}$-range and the largest length scales $(O(\delta))$. They argue that this new regime is characterized by a constant value of $\left\langle\left[\Delta u_{x}^{\prime}\right]^{2}\right\rangle$ which is of the order of $U_{0}^{2}$ and therefore $\left\langle\left[\Delta u_{x}^{\prime}\right]^{2}\right\rangle \propto U_{0}^{2}$. In other words, in this regime, all eddies have a similar kinetic energy which is of the order of $U_{0}^{2}$ [32]. The transition of the two ranges is estimated to occur at $r \sim z$. These arguments for the 2 nd order structure function $\left\langle\left[\Delta u_{x}^{\prime}\right]^{2}\right\rangle$ of course hold similarly for the kinetic energy spectrum $E(k)$ and equally for the longitudinal energy spectrum. More precisely, for wave numbers sufficiently larger than $2 \pi / \delta$ but still relatively small (lets say $k<2 \pi / z$ ), a scaling of

$$
E_{x}\left(k_{x}\right) \sim U^{2} k_{x}^{-1}
$$

is expected, whereas for wavenumbers much smaller then $s \pi / \eta$ but larger than lets say $k>2 \pi / z$

$$
E_{x}\left(k_{x}\right) \sim \varepsilon^{2 / 3} k_{x}^{-5 / 3}
$$

is expected.

Superstructures. When speaking about the turbulent boundary layer it is also important to mention the large-scale turbulence structures. These structures have been observed in a variety of wall bounded flows, such as in atmospheric boundary layers $[33,34]$, pipe flows [35], or channel flows [36, 37]. Much longer streamwise momentum structures named superstructure have been detected not only, but also in wall bounded flows [38-42].

An example of such structures is shown in fig. 2.5, where a snapshot of the streamwise velocity fluctuations are shown. While the flow is very irregular, it is visible that the spanwise dimension is about 10 times smaller than their extent in the streamwise direction. These structures are probably caused by larger vortex structures that transport slow fluid from the near-wall region upwards, i.e., in wall-normal direction (blue regions), while fast flowing fluid is transported by the same vortices from the bulk towards the plate (red region). While fig. 2.5 shows a snapshot of the two-dimensional velocity field, the turbulent superstructures are also visible in pointwise velocity measurements (e.g., 


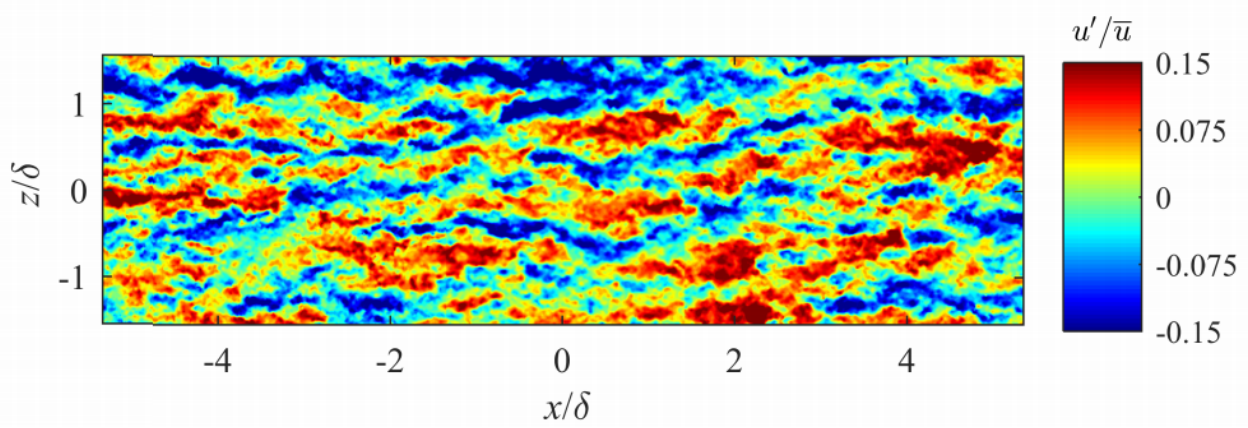

Figure 2.5: Experimental investigation of turbulent superstructures in flat plate with zero pressure gradient. Image from Eich 2017 [43]

via hot-wire measurements), as a 2nd maximum that grows in profiles of the velocity fluctuations $u^{\prime}(z)$. For example, in local measurements of the velocity in the atmospheric boundary layer the superstructures were detected as rather regular and low-frequency fluctuations with periodicity in a range between 1 and 10 minutes. These large and superstructures has been observed to have different characteristics from the usual classical turbulent fluctuations [34], [44], [45], [46].

\subsubsection{The transition from a laminar to a turbulent boundary layer}

O.Reynolds (1883) [5] has studied the transition from laminar to turbulent flows in pipes. He also realised that the ratio between inertia and viscosity damping plays a crucial role in the determination of the flow condition and he introduced the dimensionless number that now carries his name. In particular, Reynolds found that the flow was laminar when the Reynolds number was below a certain threshold $\mathrm{Re}_{\text {crit }}$. If $\mathrm{Re}$ is raised beyond $\mathrm{Re}_{\text {crit }}$, the flow in the pipe became first unsteady and later fully turbulent. Most of the studies at the beginning of the fluid mechanic's analysis were focused on laminar-turbulent transition in the outer region of the flow, far from the boundary.

The mechanisms that lead to turbulence are not the same for all wall-bounded flows. The laminar flow in pipes that were studied originally by Reynolds is in fact linearly stable for all Re and therefore a $\mathrm{Re}_{\text {crit }}$ cannot be calculated analytically. Instead, the transition to turbulence rather occurs because the survival time (lifetime) of flow perturbations increases with increasing Re, whereas small turbulent patches can split and hence create new turbulent patches [47]. From this mechanism, it is clear that the transition 
to turbulence is affected by many other parameters next to the Reynolds number, such as pressure distribution in the outer flow, induced wall disturbances (roughness), or the initial state of the flow upstream.
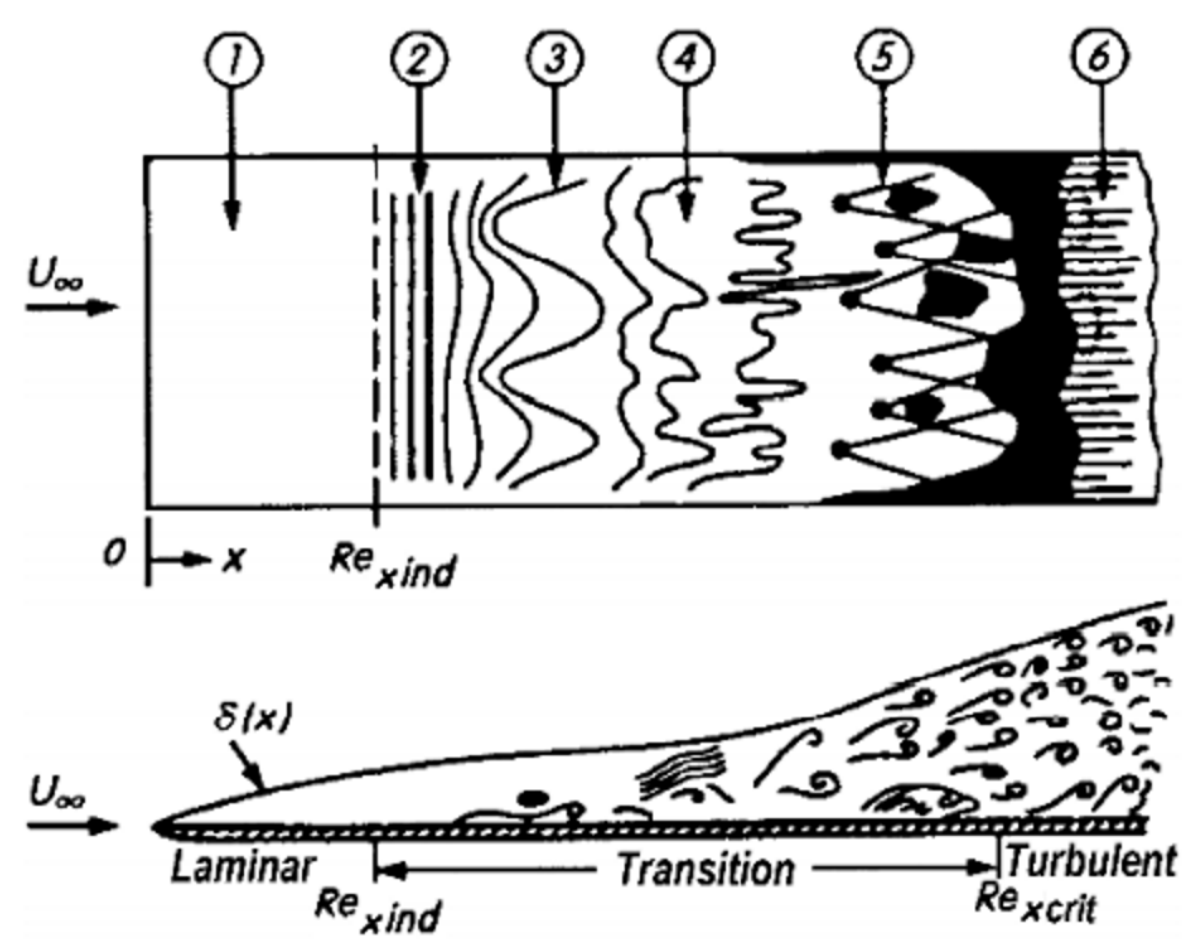

Figure 2.6: Sketch of the top and the side view of a transitional boundary layer that develops on top of a flat plate. The marked points refer to: (1) stable laminar flow, (2) unstable Tollmien-Schlichting waves, (3) three-dimensional waves and vortex formation, (4) vortex decay, (5) formation of turbulent spots, and (6) fully turbulent flow. Image adapted from [29].

Figure 2.6 shows a sketch of a boundary layer on top of a flat horizontal plate and how it turns from laminar to turbulent with increasing distance from the leading edge. For a horizontal flat plate, the boundary layer that starts to develop at $\mathrm{x}=0$ will be laminar initially and will therefore grow as $\delta(x) \propto \sqrt{x}$ as shown in sec. 2.3.1 and with a vertical velocity profile as described by the Blasius equation (eq. 2.11). At some location $x$, the Reynolds number has reached a critical value $\mathrm{Re}_{\text {ind }}$ so that the laminar boundary layer becomes unstable and turns turbulent.

For $R e_{x}<R e_{i n d}$, the flow inside the boundary layer is stable because small perturbations are dampened by viscous diffusion. For $R e_{\text {ind }}<R e_{x}<R e_{\text {crit }}$ we are in the transitional regime where the flow becomes unstable, and all the perturbations are amplified along with the flow, but the system is not yet fully turbulent. The most unstable modes are oscillations with wave vector parallel to the flow direction, the so-called 
Tollmien-Schlichting waves [48]. For $R e_{x}>R e_{\text {crit }}$ the boundary layer is fully turbulent.

The first experimental investigation of the transition to turbulence for such a boundary layer has been conducted already a century ago by Burgers [1], Van der Hegge Zijnen [2], Hassen [3], and Dryden [4], who measured the critical Reynolds number to be in the range $3 \times 10^{5}<R e_{\text {crit }}<10^{6}$. Schubauer and Klebanoff in 1955 [49] have measured the turbulent boundary layer transition in a wind-tunnel with an extremely low free flow turbulence intensity, measuring the turbulent transition somewhere in the range $3 \cdot 10^{6}<\operatorname{Re}_{\text {crit }}<4 \cdot 10^{6}$. Note that the transition does not only depend on $\operatorname{Re}_{x}$ but also on other factors such as the external flow turbulence, surface roughness or small inhomogeneities, which explains the rather large range of experimentally measured $\mathrm{Re}_{\text {crit }}$.

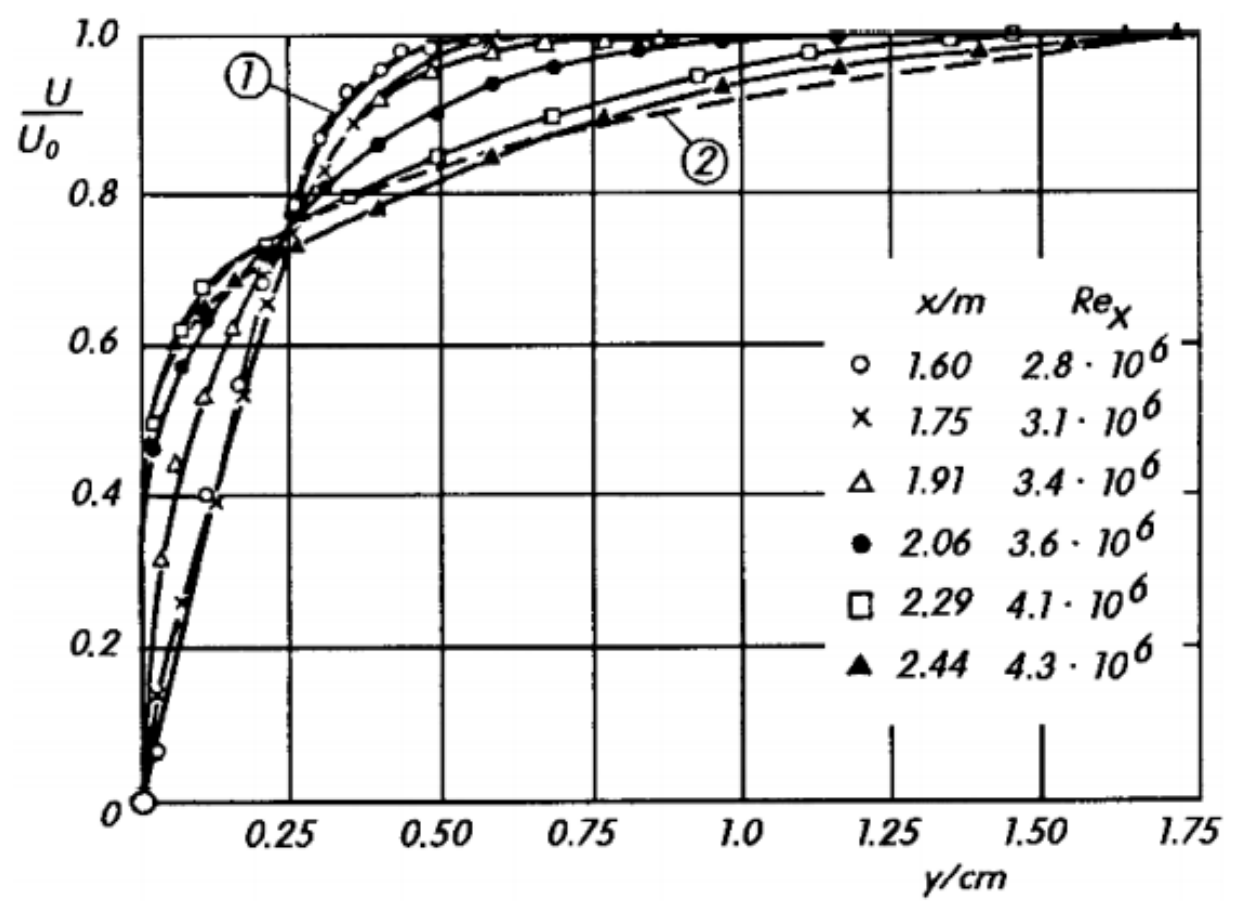

Figure 2.7: Experimental investigation averaged streamwise velocity profiles in a boundary layer for $R e_{x}$ ranging from the laminar (1) to turbulent regime (2). Image from [48] p.422, adapted from [49]

The change of the velocity profile inside a boundary layer when it transits from laminar to turbulent is shown in fig. 2.7. The figure is from [48] and shows measurements by Schubauer [49]. When the flow is laminar (1 in fig. 2.7), the average streamwise velocity resembles the already shown Blasius profile (eq. 2.11), which is a nearly linear velocity gradient that decreases to zero towards the top of the boundary layer. The profile of the average velocity in a turbulent boundary layer (2 in fig. 2.7) is qualitatively different. The 
initial velocity gradient is significantly large, i.e., the curve is steeper, than for the Blasius profile. However soon, at around $y \approx 0.2 \mathrm{~cm}$ the velocity profile flattens and follows the typical logarithmic profile. As a result of the flatter section, the boundary layer height is much larger than for the laminar case. Figure 2.7 clearly shows that the transition from a laminar to a turbulent profile is rather smooth and continuous without sudden qualitative changes.

To better understand this transition some experimental studies such as [49-51] investigated the growth of turbulent spots inside the boundary layer. Figure 2.8 is adapted from the [49], it shows the velocity fluctuations as a function of time, for different distances from the leading edge $x$, hence different Reynolds numbers $\operatorname{Re}_{x}$. In this dataset, $\operatorname{Re}_{x}=1.99 \times 10^{6}$ does still represent a laminar flow, and the velocity signal is steady in time. For increasing $\operatorname{Re}_{x}$ we observe periodic perturbations that grow with increasing $x$. For $\operatorname{Re}_{x}=2.98 \times 10^{6}$ the signal is already strongly fluctuating although the turbulence is only fully developed at $\operatorname{Re}_{x}=3.22 \times 10^{6}$.

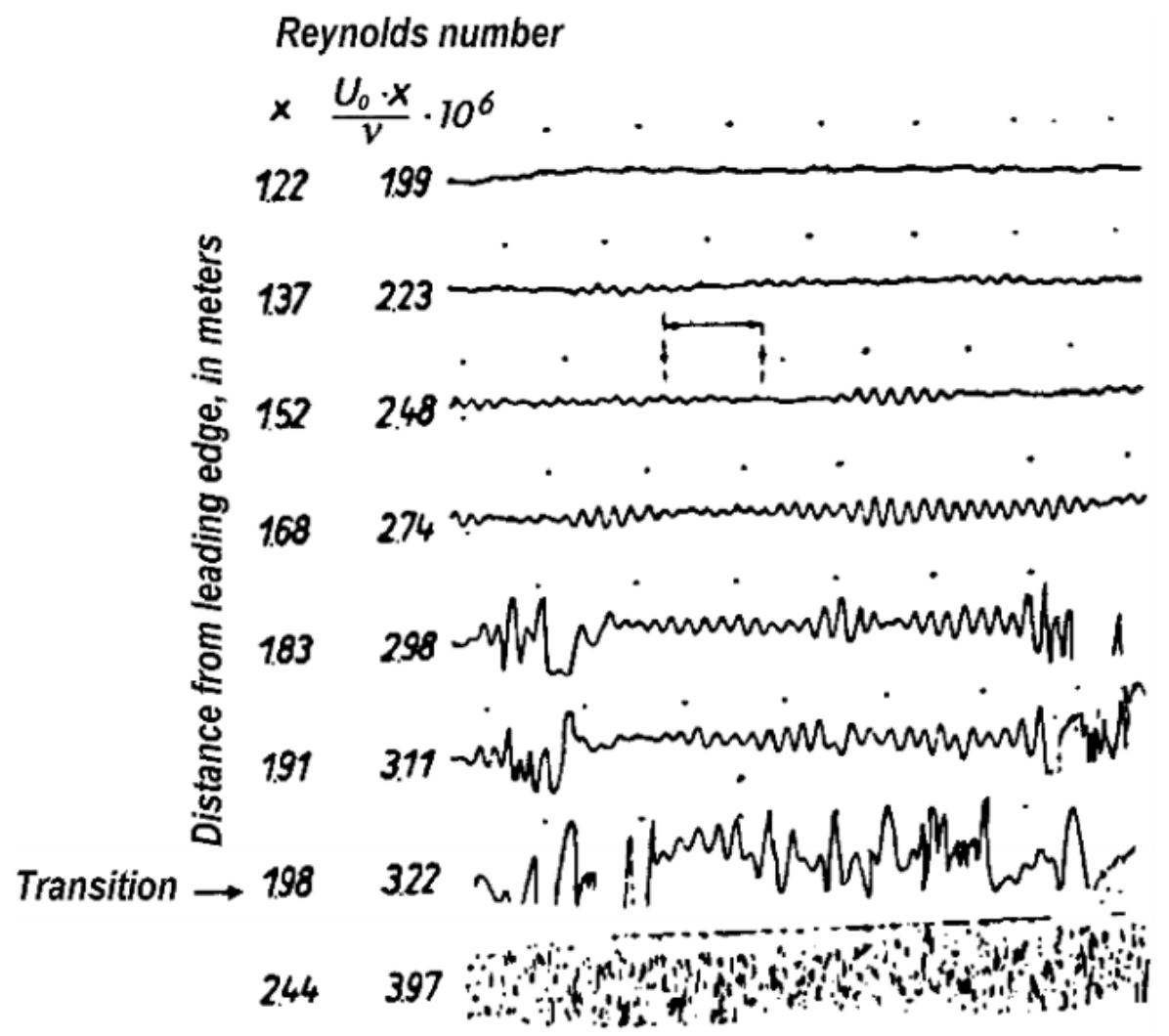

Figure 2.8: Experimental investigation of transitional flow. Shown are velocity fluctuations as a function of time for different Re. Image from [48] p.444, adapted from [51]

This transition to turbulence is characterized by the growth of non-regular velocity 
fluctuations. Those fluctuations cannot be easily predicted due to their chaotic nature and hence the sensitivity to parameters that are usually out of control, i.e. dust deposited on a surface, surface roughness and others. This led to the need for a statistical tool that allows to quantitatively represent the transition from laminar to turbulent. The parameter of choice for the authors of those studies has been called intermittency factor $\gamma$. The intermittency factor attempt to determine how long a time signal is laminar and how long it is disturbed by perturbations, the ratio between those two times can generally suggest how far is a given flow in the transition between laminar and turbulent. This will be better discussed in section 4.1.3.

We also want to mention the stability theory [48] that calculates at which Reynolds number $\mathrm{Re}_{\text {ind }}$ the laminar Blasius profile becomes unstable with respect to small amplitude perturbations. The Reynolds number at which perturbations are neutrally stable (zero growth rate), depending on their wavelength. Hence $\mathrm{Re}_{\text {ind }}$ refers to the minimum of this neutral stability curve. For $\operatorname{Re}<\mathrm{Re}_{\text {ind }}$ any given perturbation is dampened, while for

$\operatorname{Re}_{\text {ind }}<$ Re perturbations with a small range of wave numbers are amplified. Unstable perturbation is carried with the flow while they grow. Only further downstream have under such condition the flow becomes turbulent. The position, where the boundary layer is fully turbulent can be expressed by another Reynolds number $\operatorname{Re}_{\text {crit }}>\operatorname{Re}_{\text {ind }}$. Often, it is helpful to distinguish between $\mathrm{Re}_{\text {crit }}$ and $\mathrm{Re}_{\text {ind }}$. We can then consider $\mathrm{Re}_{\text {ind }}$ and $\mathrm{Re}_{\text {crit }}$ as the lower and the upper bound of the transitional regime.

\subsection{Thermal boundary layer under shear forces}

A flow over a hot surface is of crucial importance for many engineering applications and hence has been studied for over a century. In particular, many investigations have aimed to determine how much heat is transported from the hot surface into the outer flow. Despite the radiation, heat can be transported by diffusion and advection, while the latter is significantly more effective and hence of greater significance for applications. Advection itself can either be produced by buoyancy due to temperature differences or by the outer flow itself. Which of these effects is more important is expressed by the Richardson number Ri (see sec. 2.5). For very small Ri, buoyancy is small and can be neglected. Then the temperature field is advected like a passive scalar with the flow and 
does not influence the velocity field.

We will now discuss the temperature field and the heat transport through a laminar (sec. 2.4.1) and turbulent boundary layer (sec. 2.4.2) for $\mathrm{Ri} \ll 1$ when buoyancy is neglected. Later in sec. 2.4 .3 we also will discuss boundary layers with buoyancy for $\mathrm{Ri} \gg 1$.

\subsubsection{Laminar boundary layer}

The theoretical considerations for the temperature field in a laminar boundary layer presented below can be found in [48] and references therein. Let us assume the usual flat surface immersed in a flow with a relative velocity $U_{0}$, and we will assume that the flat surface is warmer than the flow above it. Now, we have a second physical quantity, namely the temperature $T(\mathbf{x}, t)$, which interacts with the velocity field. Similar to the velocity, also the thermal field can be split into two main regions, close and far from the surface. When both velocity and temperature field does exist they are usually mutually coupled.

If we assume a thermal conductivity $\lambda$ and a constant specific heat capacity $c_{p}$, for a steady flow in two dimensions, the energy equation is written as:

$$
\varrho c_{p}\left(u \frac{\partial T}{\partial x}+v \frac{\partial T}{\partial z}\right)=\lambda\left(\frac{\partial^{2} T}{\partial x^{2}}+\frac{\partial^{2} T}{\partial z^{2}}\right)
$$

Here, we have also neglected viscous dissipation. This can be done, when the dissipation rate is sufficiently small compared to the advected and conducted thermal energy.

We will first discuss flows where the temperature does not couple to the velocity field. This approximation is valid for flows with strong inertia forces and weak buoyancy. In this approximation, we can consider that all the assumptions done for the velocity field in the previous chapters hold.

We need at first to introduce dimensionless quantities for space, velocity and temperature:

$$
x^{*}=\frac{x}{L}, \quad z^{*}=\frac{z}{L}, \quad u^{*}=\frac{u}{U_{0}}, \quad v^{*}=\frac{v}{U_{0}}, \quad \vartheta=\frac{T-T_{0}}{\Delta T} .
$$

We furthermore define a Reynolds number as usual and use it to re-scale the vertical 
coordinate and the vertical velocity:

$$
R e_{x}=\frac{U_{0} x}{\nu}, \bar{v}=v^{*} \sqrt{R e_{x}}, \quad \bar{z}=z^{*} \sqrt{R e_{x}}
$$

with $U_{0}$ being the bulk velocity, $L$ a characteristic length, and $\Delta T=T_{w}-T_{0}$ the difference in temperature between the surface the outer flow. With this and under the assumption of large $\operatorname{Re}_{x}$ (the first term on the right in eq. 2.27) can be neglected) we can write eq. 2.27 dimensionless as

$$
u^{*} \frac{\partial \vartheta}{\partial x^{*}}+\bar{v}^{*} \frac{\partial \vartheta}{\partial \bar{z}}=\frac{1}{\operatorname{Pr}} \frac{\partial^{2} \vartheta}{\partial \bar{z}^{2}}
$$

with Prandtl number

$$
\operatorname{Pr}=\frac{\nu}{\alpha}
$$

The Prandtl number represents the ratio between momentum and thermal diffusion and is a physical property of the fluid. If the dissipation is neglected the eq. 2.30 can be written in dimensional form as:

$$
u \frac{\partial T}{\partial x}+v \frac{\partial T}{\partial z}=\alpha \frac{\partial^{2} T}{\partial z^{2}}
$$

with $\alpha$ being the thermal diffusivity

$$
\alpha=\frac{\lambda}{\varrho c_{p}}
$$

From the above calculations, we see that the temperature field $T(x, z)$ in the boundary layer can only be calculated when the velocity field is know. For a laminar boundary layer, we have an analytical solution for the velocity, i.e., the Blasius solution.

Let's have a look at fig. 2.9. There we consider a flat surface with a sharp leading edge at $\mathrm{x}=0$ and a bulk flow of $U_{0}$ above it with temperature $T_{0}$. The front the plate is set at $T_{0}$ for $0<x<x_{0}$ and increases to $T_{w}>T_{0}$ at $x=x_{0}$. For $x>x_{0}$ a thermal boundary layer similar to the viscous boundary layer starts to grow. While the viscous and the thermal boundary layer do not share the same spatial origin, their heights growth is given by their respective diffusivities, i.e, the Prandtl number.

The problem in fig. 2.9 can easily be solved numerically. However, we do not need to know the exact temperature field to make some important statements about the heat transport from the bottom plate $q_{w}(x)=-\left.\lambda \frac{\partial T(x)}{\partial z}\right|_{z=0}$. To make this statement more 


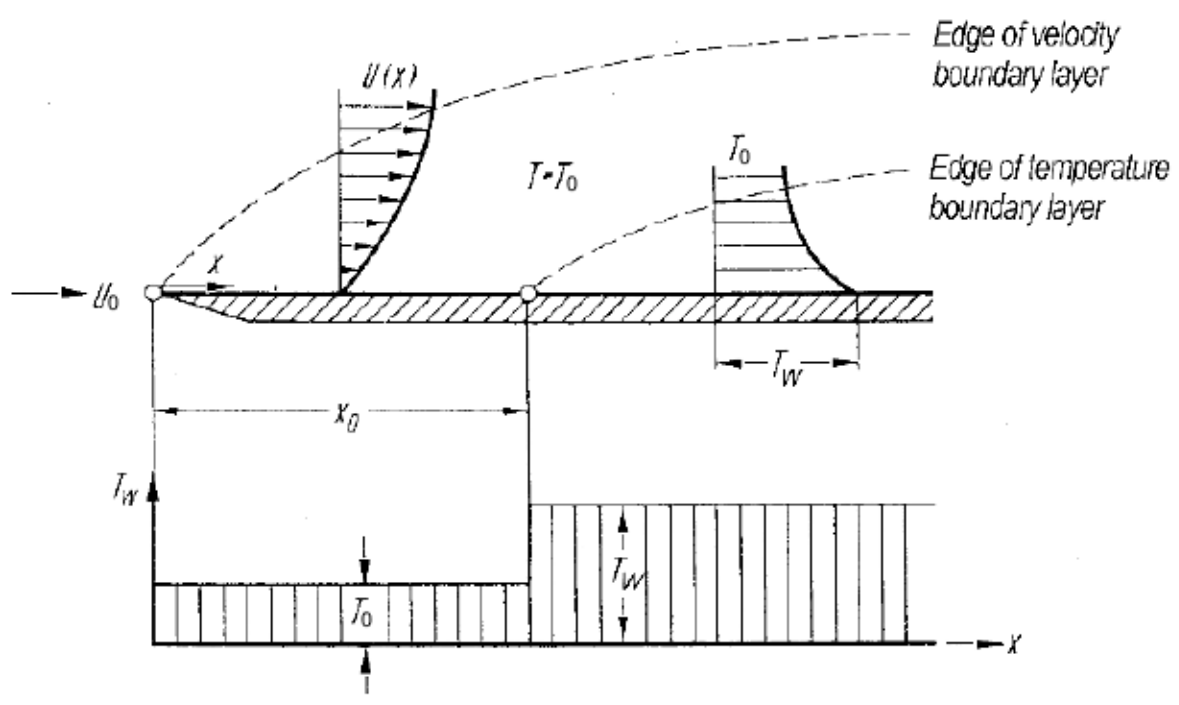

Figure 2.9: Development of the velocity and temperature boundary layer distribution with wall temperature position different from leading edge Image ref. [29]

general lets define a Nusselt number as

$$
\mathrm{Nu}_{x}(x)=\frac{q_{w}(x) x}{\lambda\left[T_{w}(x)-T_{0}\right]}=\sqrt{\mathrm{Re}_{x}} \cdot \frac{\partial \vartheta(x)}{\partial \bar{z}}
$$

For the term on the right, we have replace $q_{w}$ with the temperature gradient at $z=0$ but have re-scaled $z$ to $\bar{z}$ and $T$ to $\vartheta$ according to 2.29. From eq. 2.30, $\vartheta$ only depends on $\operatorname{Pr}$ but is independent of $\operatorname{Re}_{x}$. Therefore, we can state that the Nusselt number is proportional to the square root of the Reynolds number:

$$
\mathrm{Nu}_{x} \propto \sqrt{\operatorname{Re}_{x}}
$$

Note, that this relation appears as an asymptotic behaviour for Reynolds numbers that are sufficiently large so that viscous shear is larger than buoyancy, but still below $\operatorname{Re}_{\text {ind }}$ at which the laminar boundary layer becomes unstable.

\subsubsection{Turbulent boundary layer}

By keeping in mind the considerations done for the laminar boundary layer, we want to derive the thermal boundary layer equations for a turbulent boundary layer. The derivation is suggested by Kader and Yaglom (1972) [23].

We consider a heated flat surface and a turbulent heat transfer from it. When buoyancy 
is neglected, heat is transported by the same turbulent eddies that also carry momentum. Therefore, in analogy to the skin friction coefficient $c_{f}$, we define a heat transfer coefficient, also know as the Stanton number:

$$
c_{h}=\frac{q_{w}}{c_{p} \rho U_{0}\left(T_{w}-T_{0}\right)}
$$

Here, $q_{w}$ represents the heat flux from the surface, $T_{w}$ is the surface temperature, $T_{0}$ is the outer flow temperature, and $c_{p}$ the specific heat capacity. As one easily sees from eq. 2.34 and eq. 2.36, the Stanton number and the Nusselt number are related:

$$
N u=c_{h} \operatorname{Re} \operatorname{Pr}
$$

This relationship is called heat transfer law. In analogy to the skin friction velocity, Obukhov (1946) [52], H.B. Squire in 1951 [53] introduced the friction temperature:

$$
T_{\tau}=\frac{q_{w}}{c_{p} \rho u_{\tau}}
$$

In the wall layer close to the surface $y \ll \delta$ the dimensional arguments yield us to the following diffusion wall law, which is analogous to the law of the wall eq. 2.17:

$$
T_{w}-T(z)=T_{\tau} \varphi\left(\frac{u_{\tau} z}{\nu}, \operatorname{Pr}\right)
$$

It is also true that in the outer region the result should be independent of the molecular constants and hence similar to the von Karman velocity deficit law (2.18) and therefore we can write:

$$
T(z)-T_{0}=T_{\tau} \varphi_{1}\left(\frac{z}{\delta}\right)
$$

For a sufficiently turbulent boundary layer, a region should exist where both functions overlap. This results in the log-law for the temperature in this region:

$$
\frac{T_{w}-T\left(z^{+}\right)}{T_{\tau}}=\alpha \ln z^{+}+\beta(\operatorname{Pr})
$$

For the derivative of eq. 2.41, one also had to consider that the temperature drop across the entire boundary layer could only be a function of $\left(\delta u_{\tau} / \nu\right)$ and Pr. It is the same 
assumption that leads after some calculations (see [23] for details) to an expression for the Stanton number as function of the skin friction coefficient and the Reynolds number:

$$
c_{h}=\frac{\sqrt{\left(c_{f} / 2\right.}}{\alpha \ln \left(R e_{x} c_{f}\right)+\gamma},
$$

with $\alpha$ and $\gamma$ unknown constants that can be derived with experiments. For a flat plate, $\alpha \approx 3.04 / \operatorname{Pr}$ and $\gamma=2.68 \operatorname{Pr}$ were found. For not too large $\operatorname{Re}_{x}\left(<10^{7}\right)$, eq. 2.42 can be approximated well by:

$$
c_{h}=\frac{0.0296}{\operatorname{Re}_{x}^{0.2} \operatorname{Pr}^{0.4}} \quad \text { or } \quad \mathrm{Nu}_{x}=0.0296 \operatorname{Re}_{x}^{0.8} \operatorname{Pr}^{0.6}
$$

\subsubsection{The influence of heat on the velocity boundary layer}

\section{Temperature dependent fluid properties}

Up to now, we have only considered heated boundary layers, where the change of temperature does not affect the physical properties of the fluid. In general, however, the fluid properties, such as the viscosity $\mu$, the heat conductivity $\lambda$, or the heat capacity $c_{p}$ are functions of the temperature. Most importantly, the density decreases for most fluids with temperature (for gases $\rho \propto T^{-1}$ ) which leads to buoyancy, an additional driving force for the fluid. Before we discuss buoyancy in sec. 2.4.3, we first quickly consider a boundary layer where buoyancy is still rather small compared to inertial, viscous or pressure forces, but where the change of the fluid properties already alter the dynamic of the boundary layer significantly.

For a boundary layer that occurs in a flow of temperature $T_{0}$ over a heated plate of temperature $T_{w}$, important response parameters, such as the skin friction coefficient or the heat transport can be calculated via correction factor from the skin friction or the heat transport in the case of constant fluid properties. These correction factor are calculated from power laws of the fluid properties ratios evaluated at the flow temperature and the plate temperature. Concretely, for the skin friction coefficient, the Nusselt number (for uniform wall temperature) or the wall temperature (for uniform wall heating) one can write (according to [48]) a relation between them and their values assuming constant 
fluid properties (index "c"):

$$
\begin{aligned}
\frac{c_{f}}{c_{f_{c}}} & =\left(\frac{\rho_{w} \mu_{w}}{\rho_{0} \mu_{0}}\right)^{m_{\rho \mu}}\left(\frac{\rho_{w}}{\rho_{0}}\right)^{m_{\rho}} \\
\frac{\mathrm{Nu}}{\mathrm{Nu}_{c}} & =\left(\frac{\rho_{w} \mu_{w}}{\rho_{0} \mu_{0}}\right)^{n_{\rho \mu}}\left(\frac{\rho_{w}}{\rho_{0}}\right)^{n_{\rho}}\left(\frac{\operatorname{Pr}_{w}}{\operatorname{Pr}_{0}}\right)^{n_{P r}}\left(\frac{c p_{w}}{c p_{0}}\right)^{0.5} \\
\frac{T_{w}-T_{0}}{\left(T_{w}-T_{0}\right)_{c}} & =\left(\frac{\rho_{w} \mu_{w}}{\rho_{0} \mu_{0}}\right)^{k_{\rho \mu}}\left(\frac{\rho_{w}}{\rho_{0}}\right)^{k_{\rho}}\left(\frac{\operatorname{Pr}_{w}}{\operatorname{Pr}_{0}}\right)^{k_{P r}}\left(\frac{c p_{w}}{c p_{0}}\right)^{0.5} .
\end{aligned}
$$

Here, the index " $w$ " denotes fluid properties evaluated at the wall temperature $T_{w}$, and the index "0" denotes fluid properties evaluated at the bulk flow temperature $T_{0}$. The exponents depend on $\operatorname{Pr}$ and have been determined semi-empirically. For $\operatorname{Pr}=0.7$ and $q_{w}$ they are $m_{\rho \mu}=0.313, k_{\rho \mu}=-0.255, k_{P r}=0.389, m_{\rho}=k_{\rho}=0$ (see pg. 239 in [48]).

\section{The influence of buoyancy}

The influence of buoyancy on a turbulent shear boundary layer is of particular interest in atmospheric sciences. There, the atmospheric boundary layer that develops due to large scale winds is influenced by buoyancy, which depends on the time of the day. During nights the Earth surface is colder than the air above and hence buoyancy has a stabilising effect, while during the day, the Earth surface heats up due to solar radiation, becomes warmer than the air above, creating an unstable situation. Then, the fluid near the surface is warmer and hence less dense than the fluid above. In this way, potential energy is introduced into the system that is converted into kinetic energy and subsequently adds to the turbulent kinetic energy of the boundary layer.

By comparing the creation of turbulent kinetic energy due shear with the turbulent kinetic energy due to buoyancy, Monin and Obukhov 1954 [54] and again Obukhov in 1971 [55] have defined a flux Richardson number:

$$
\operatorname{Ri}_{f}=\frac{g}{T_{0}} \frac{\overline{T u_{z}^{\prime}}}{\left(\overline{u_{x}^{\prime} u_{z}^{\prime}} \partial \overline{u_{x}} / \partial z\right)}
$$

where the overlines $\ldots$ denotes averages in time, $g$ the gravitation acceleration, $T^{\prime}$ the temperature fluctuations, and $u_{x, z}^{\prime}$ the velocity perturbation. ${ }^{3} \operatorname{Ri}_{f}$ is a function of $z$.

\footnotetext{
${ }^{3}$ In atmospheric sciences often $\mathrm{Ri}_{f}$ is defined with a minus in order to distinguish an destabilising from a stabilising buoyancy flux. Since here we only consider an unstable boundary layer that is heated from below, we omit the sign.
} 
While one can assume that the vertical heat flux $q_{T}=\overline{\rho c_{p} T u_{z}^{\prime}}$ is rather independent of $z$, this assumption is certainly wrong for the production of kinetic energy due to shear $\left(\overline{u_{x}^{\prime} u_{z}^{\prime}} \partial \overline{u_{x}} / \partial z\right)$. However, as shown in sec. 4.2.1, in the log-region, the average velocity profile is well approximated by $\partial \overline{u_{x}} / \partial z=u_{\tau} /(k z)$ and hence eq. 2.47 can be written as

$$
\operatorname{Ri}_{f}=\frac{z}{L_{m}} \quad \text { with } \quad L_{m}=\frac{\left(T_{0} / g k\right) u_{\tau}^{3}}{\left(q_{T} / \rho c_{p}\right)}
$$

being the Monin-Obukhov length scale. We see now that for $z \ll L_{m}$, it is $\mathrm{Ri}_{f} \ll 1$ and the flow is dominated by shear turbulence. For $z \gg L_{m}$, or $\mathrm{Ri}_{f} \gg 1$, the flow is dominated by buoyancy.

We can only say that when $L$ is big enough it is possible to assume a similarity between the velocity boundary layer profile and the thermal boundary layer profile. To the best of our knowledge, there are no satisfying theoretical models that allow us to predict and describe the temperature profile for buoyancy dominated boundary layer or in the mixed regime.

\subsection{A note on dimensionless parameters and length scales}

It is important to discuss how the choice of the length scale may change the quantity we are studying in our system. In our experiment, we have an adiabatic plate in front and therefore the evolution of the thermal boundary layer does not coincide with the evolution of the velocity boundary layer. While without heating the distance from the leading edge $x$ was a natural choice for the typical length scale in the system, it is not expected that a dimensionless response parameter that includes the temperature or buoyancy scales with the same $x$ equally good. It is not trivial to find a good length scale so that one finds simple scaling relationships between dimensionless control and response parameters.

For example, heat transport is usually expressed by the dimensionless Nusselt number $\mathrm{Nu}$. In Rayleigh-Bénard convection, $\mathrm{Nu}=q L / \lambda \Delta T$ is defined as the ratio between the total heat transfer $q$ and the conductive heat transfer $\lambda \Delta T / L$ and the length scale $L$ is the distance between the warm bottom and the cold top plate. On a flat surface, it is a common approach to define the length scale as the distance from the leading edge $x$. 
When we use $x$ as the length scale for the Nusselt number $N u$ we no longer measure the ratio between the total and the conductive heat transfer, but we are rather comparing the heat transfer with a quantity that is proportional to $x$. Clearly, comparing dimensionless control (e.g., Gr, $\mathrm{Re}$ ) and response parameters $(\mathrm{Nu})$ is only meaningful when they are based on the same length scales. When $x$ is used as length scale for $G r$ or $R a$ we are implying that the buoyant forces a proportional to the cube of a horizontal length scale $\left(x^{3}\right)$, instead of a vertical distance as in Rayleigh-Bénard convection. To proper quantify the effect of the buoyancy the use of the thermal boundary layer height as a typical length could be a solution, but this quantity is in fact not a response parameter and hence needs to be measured first, or derived from models.

In this work, unless stated otherwise, we will use the distance from the leading edge $x$ as the typical length scale for all relevant dimensionless parameters. In this way, we assure that control parameter only depend on physical properties that can be set directly in the experiment.

We want to end this chapter with a short summary of the most important dimensionless quantities that are used in this work in their general form. The Reynolds number $(\mathrm{Re})$ [5] has been introduced right at the beginning of this chapter. It represents the ratio between the inertial and viscous forces and is the only control parameter that occurs in the dimensionless incompressible Navier-Stokes equation without additional body forces. We do show the definition for flat surface approximation:

$$
\operatorname{Re}=\frac{u L}{\nu}
$$

When the temperature is advected by the velocity field, but when the temperature does not couple to the velocity field via buoyancy, the 2nd control parameter that then occurs in the heat equation is the Prandtl number $(\mathrm{Pr})$, which represents the ratio between the momentum diffusivity and the thermal diffusivity:

$$
\operatorname{Pr}=\frac{\mu c_{p}}{\lambda}
$$

with $\lambda$ thermal conductivity, $c_{p}$ specific heat, $\mu$ dynamic viscosity. While we have not discussed it in detail above, we want to mention here that the introduction of buoyancy 
due to variations in density appears as a body force of the form $g \beta\left(T-T_{0}\right) \hat{z}$ in the NavierStokes equation. Dimensionless, the buoyancy is described by the Grashof number $(G r)$ :

$$
G r=\frac{g \beta \Delta T L^{3}}{\nu^{2}}
$$

or the Rayleigh number:

$$
\operatorname{Ra}=\mathrm{Gr} / \operatorname{Pr} .
$$

Here it is $g$ the gravitation acceleration, $\beta$ the coefficient of thermal expansion, $\Delta T$ a relevant temperature difference (in our case the temperature difference between the surface temperature and flow temperature), $L$ is a characteristic length, and $\nu$ the kinematic viscosity. The difference between free, forced or mixed convection is given be the strength of buoyancy compared to the inertial forces. The ratio of both is called the Richardson number $(R i)$ does represent the ratio between the buoyancy and the shear forces and is defined as:

$$
\mathrm{Ri}=\frac{g \beta \Delta T}{U_{0}^{2} / L}=\frac{G r}{R e^{2}}
$$




\section{Chapter 3}

\section{Experimental setup and methods}

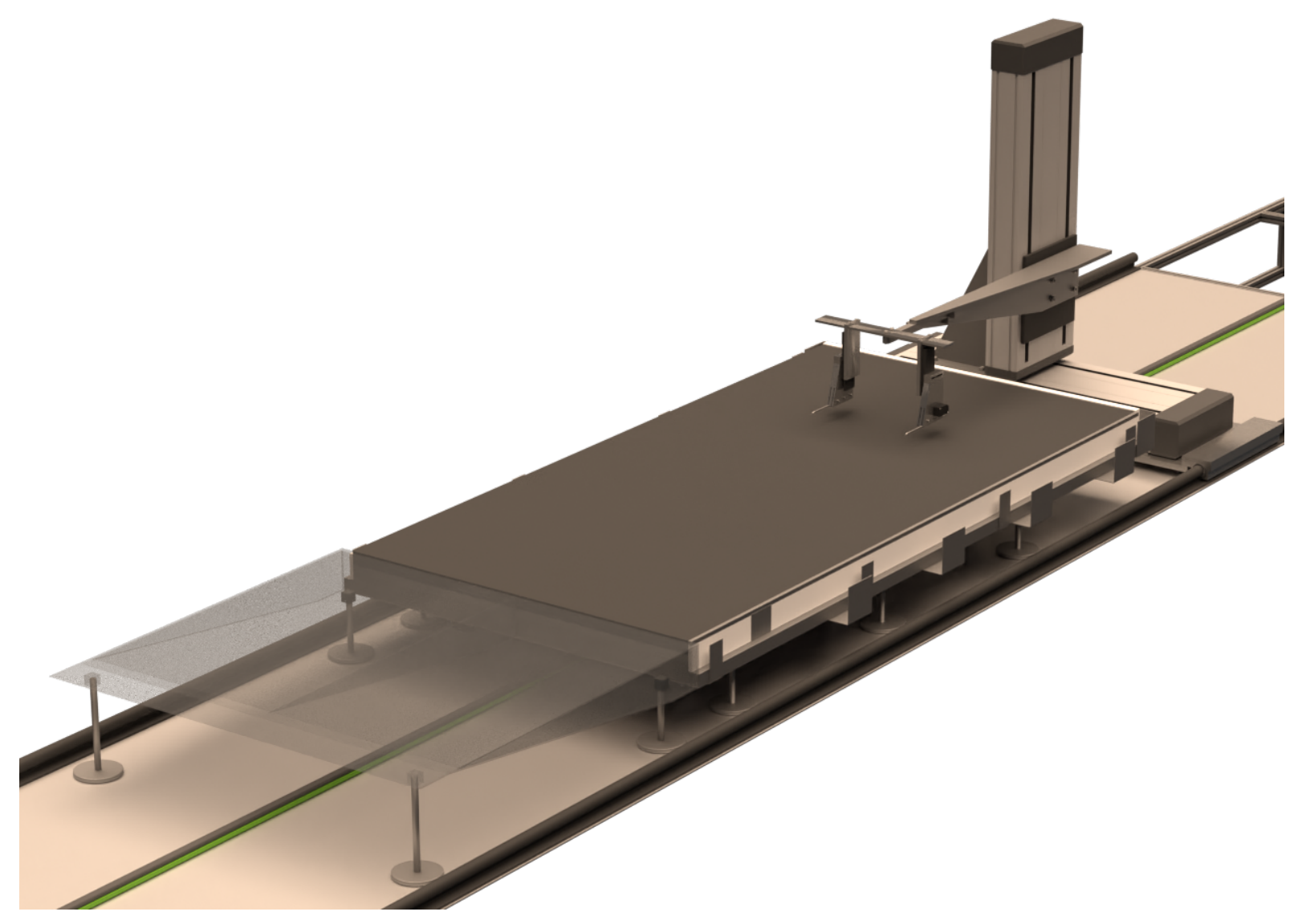

Figure 3.1: 3D rendering generic overview of the experimental setup

The experiments are carried out in the Prandtl wind-tunnel (PWT) at the Max Planck Institute for Dynamics and Self-Organization. To characterize the mixed convection, we designed and built an experiment that let us control two of the main parameters, i.e., flow velocity $U$ and temperature $\Delta T$. We can then control $G r$, and $R e$. In the PWT we 
do not have control of the gas we are using, we are limited to air. Therefore the Prandtl number is fixed to $\operatorname{Pr}=0.7$ and cannot be changed.

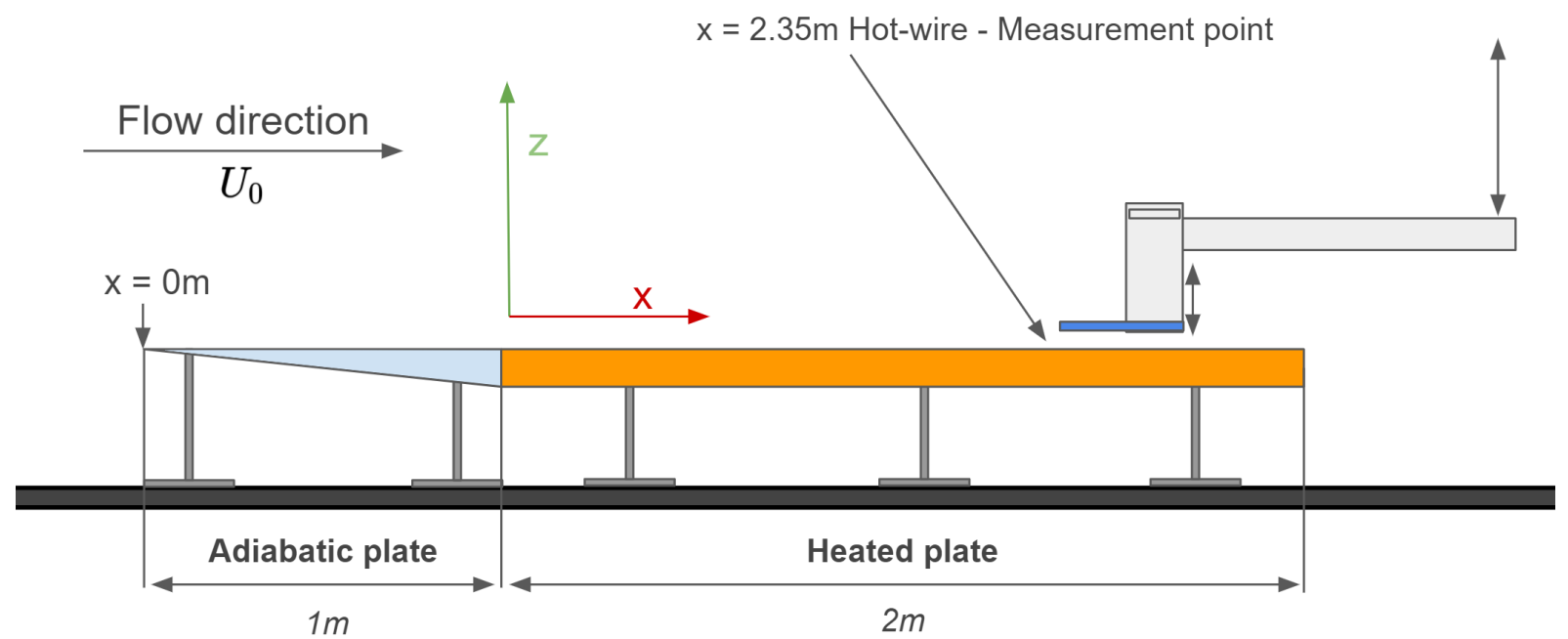

Figure 3.2: Schematic of the experiment

Figure 3.2 shows a schematic of the experimental setup. It consists of a uniformly heated plate inside a wind-tunnel. We can control the heat input in the system and control the amount of shear stress over the surface.

The incoming cold flow will encounter at first an adiabatic plate, that is 1 meter long and 1 meter wide and has a wedge-like profile. This adiabatic plate exists to minimize the flow separation at the leading edge and foster the growth of a boundary layer over it. A 2 meter long and 1 meter wide plate is then positioned in direct contact with the adiabatic plate, for a seamless surface transition. This second plate can be heated. The two surfaces are flat and smooth.

We will now discuss the experimental setup and its constituents in more detail.

\subsection{The Prandtl wind-tunnel}

The Prandtl wind-tunnel was originally called Rauhigkeitskanal, and has been built in 1935, has been revisited and reshaped in the 1970s by H. Eckelmann and J. Wallace [56] and today it is in possession of the Max Planck Institute for Dynamic and Self Organization. The PWT is an open wind-tunnel with an octagonal irregular test section. A schematic of the wind-tunnel with its different sections is shown in fig. 3.3: 
Outflow

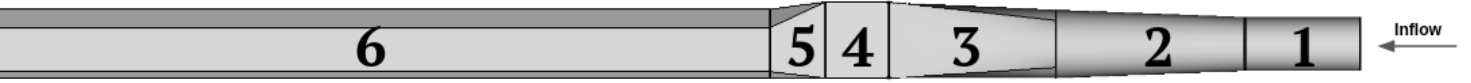

Figure 3.3: $P W T$ side view. (1) $1.45 \mathrm{~m}$ long circular section with fixed diameter of $1 \mathrm{~m}$. (2) $2.38 \mathrm{~m}$ long circular with increasing diameter from $1 \mathrm{~m}$ to $1.2 \mathrm{~m}$. (3) $2.10 \mathrm{~m}$ long square to circular shape shift. (4) $0.8 \mathrm{~m}$ long square section with the flow laminarizer grid. (5) $0.7 \mathrm{~m}$ long square to octagonal shape shift section. (6) $9.7 \mathrm{~m}$ long octagonal shape section.

The flow is driven by a direct current electrical motor, controlled by a "Siemens Touch Control panel". The motor can provide rotation rates between 1 and $1500 \mathrm{rpm}$ and is via a shaft to an 8 blades propeller. For sufficiently large rotation rates, the flow velocity increases proportionally to the rotation rates of the propeller as shown in fig. 3.5.

The propeller is located in the middle of section 1 . The extremely turbulent flow is then passed through a set of laminarizing grids. The 3 grids consist of metal meshes of decreasing mesh sizes of $2 \mathrm{~cm}, 0.35 \mathrm{~mm}$, and $0.15 \mathrm{~mm}$ that break the turbulent eddies, cause and additional vortex stretching and in this way re-laminarize the flow before it reaches section 5 , and 6 . Section 3 and 5 are shape adapter sections. These sections have been designed for a smooth transition between the different shapes of the corrective sections. Section 6 is the test section of the Prandtl wind-tunnel. It is nearly $10 \mathrm{~m}$ long, has an octagonal cross-section and is the hosting section for our experiment.

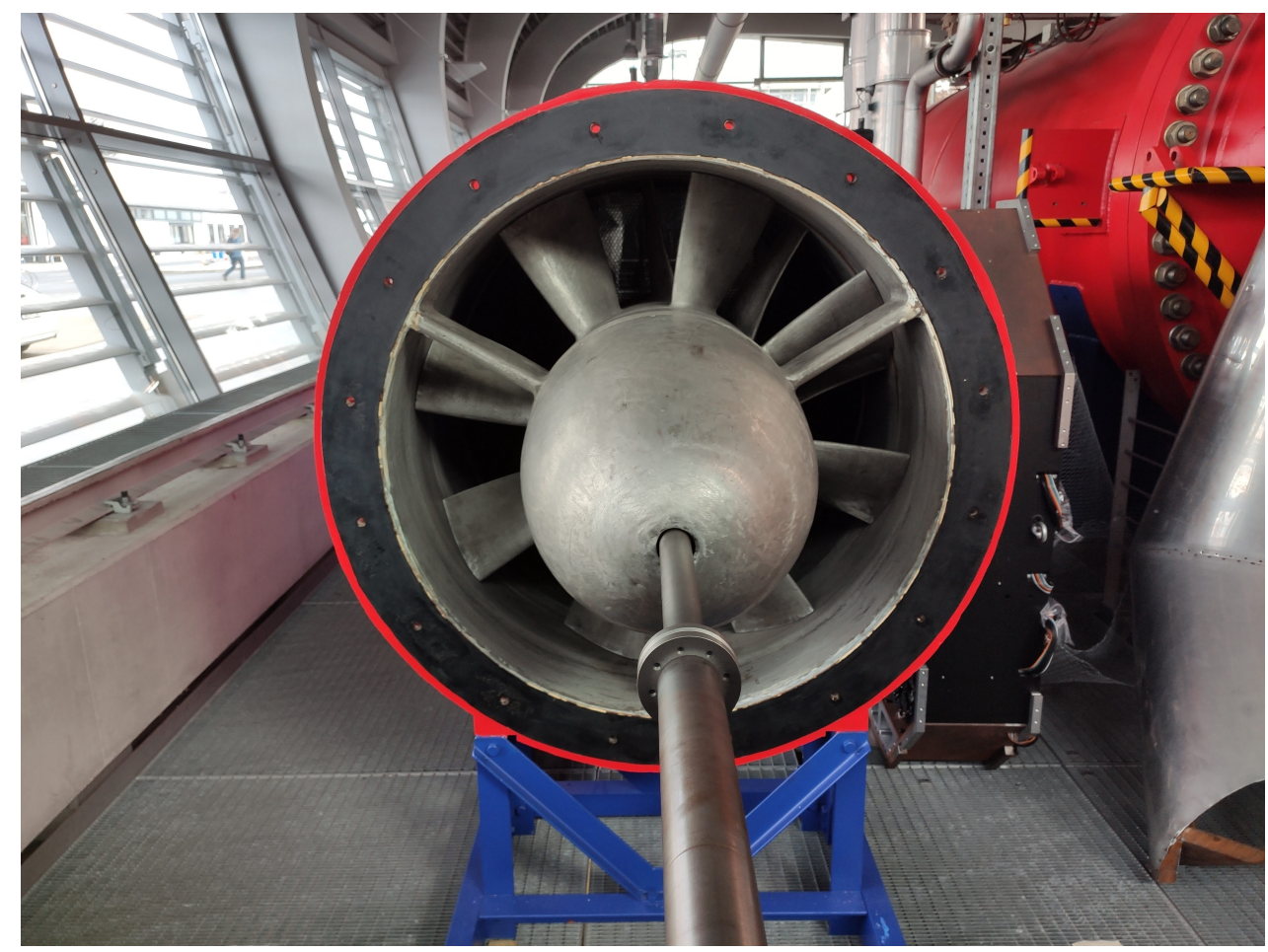

Figure 3.4: Propeller and shaft of the Prandtl wind-tunnel 
The octagonal cross-section is because of previous experiments that were performed inside the wind-tunnel. At the bottom, the test section is $1.15 \mathrm{~m}$ wide and the distance between the base and roof of the wind-tunnel is $1.25 \mathrm{~m}$. The width wall to wall is $1.5 \mathrm{~m}$. The potential effects of the unique shape of section 6 have been evaluated, and we concluded that the octagonal section should not result in strong differences from the more classical wind-tunnel section shapes. All the surfaces of this section are made out of $1 \mathrm{~mm}$ thick aluminium sheets. To maintain the $500 \mathrm{~kg}$ heavy installation of our experiments, we mounted $6 \mathrm{~mm}$ thick steel reinforcement plates on the floor of the test-section over its entire length. The $6 \mathrm{~mm}$ thick steel floor granted us a strong working surface and we achieved a negligible bending of the experimental surface.

The PWT velocity is controlled through the selection of the rotation rate of the electrical engine. The relation between the set rotation rate of the propeller (in rotations per minute - RPM) and the real velocity of the flow was measured using hot-wires probes (described in chap. 3.3). A calibration curve is shown in fig. 3.5. Note, that we have done the calibration for the same velocities that we also later used in the experiment.

While there is a nearly linear relation between the propeller rotation rate and the bulk velocity of the flow $U_{0}$, our measurements did not rely on a fitted function between these quantities. The colour code in fig. 3.5 represents the date at which the calibration has been performed. Although the engine can run at very low rotation rates, we do have to consider that the low number of blades may induce low-velocity pressure fluctuations and therefore a non-uniform fluid flow in time, which we measured as a sweet spot for the minimum velocity value around 50 RPM. Given this constrain, the PWT can produce bulk wind speeds between $0.1 \mathrm{~m} / \mathrm{s}$ and $12 \mathrm{~m} / \mathrm{s}$.

The PWT has a simple design that does not compensate for wall boundary layer growth. Therefore has been important to collect the PWT velocity profile at different locations and directions. In fig. 3.6 we show measurements of the bulk velocity collected at different locations to measure how the wall boundary layer growth affects the pressure and velocity gradient in the wind-tunnel along the streamwise and wall-normal direction. We do consider the $\mathrm{x}$-axis to be in the direction of the flow and the z-axis to be the vertical axis, normal to the bottom plate. In the plots in fig. 3.6, we have assumed the position $x=0$ to be at the intersection between section 5 and 6 , whereas $x$ increases in the flow direction. Furthermore, $z=0$ marks the floor of the wind-tunnel and $\bar{U}$ represents the 


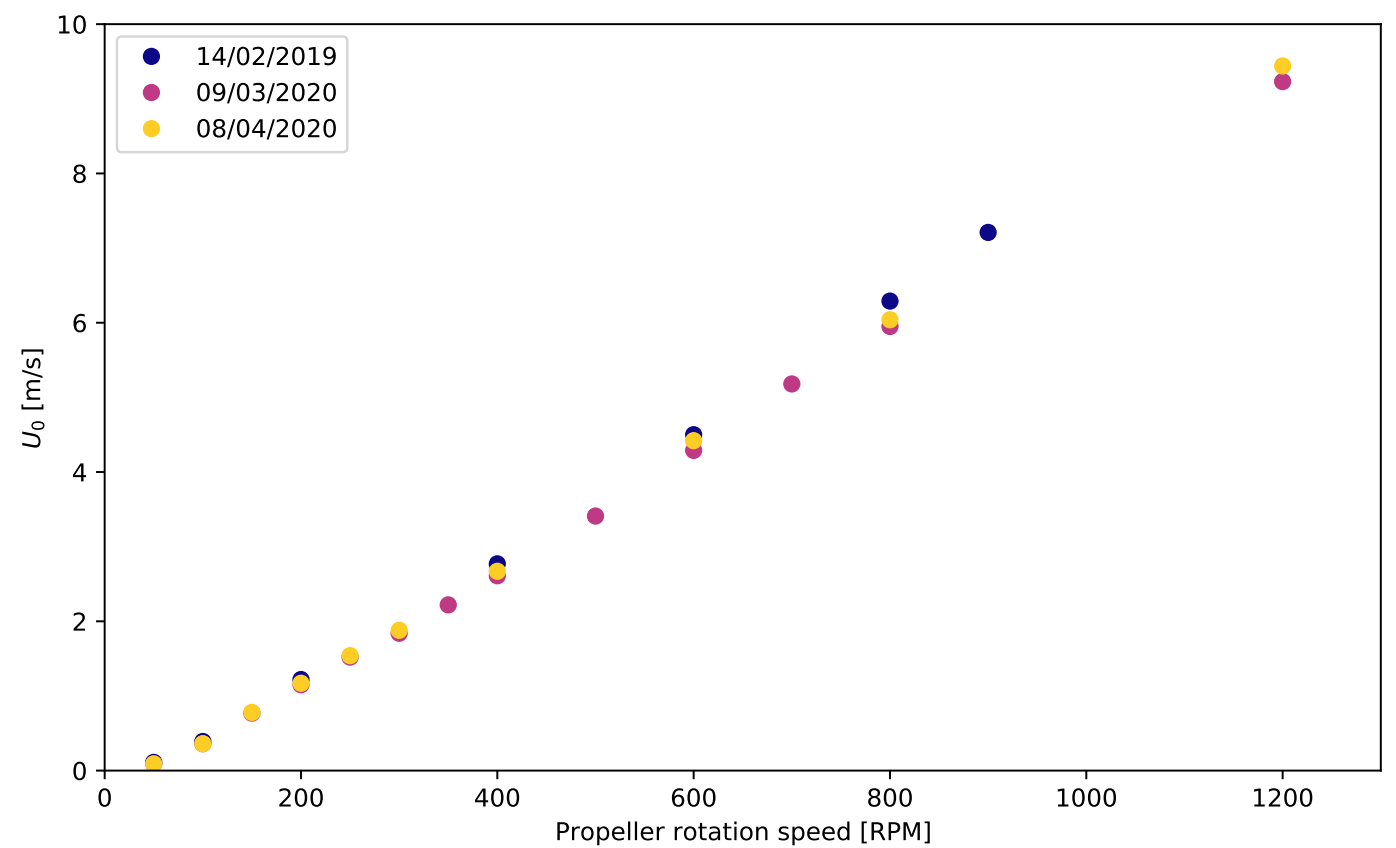

Figure 3.5: PWT velocity calibration. Measurement is done in the bulk at mid-height of the tunnel.
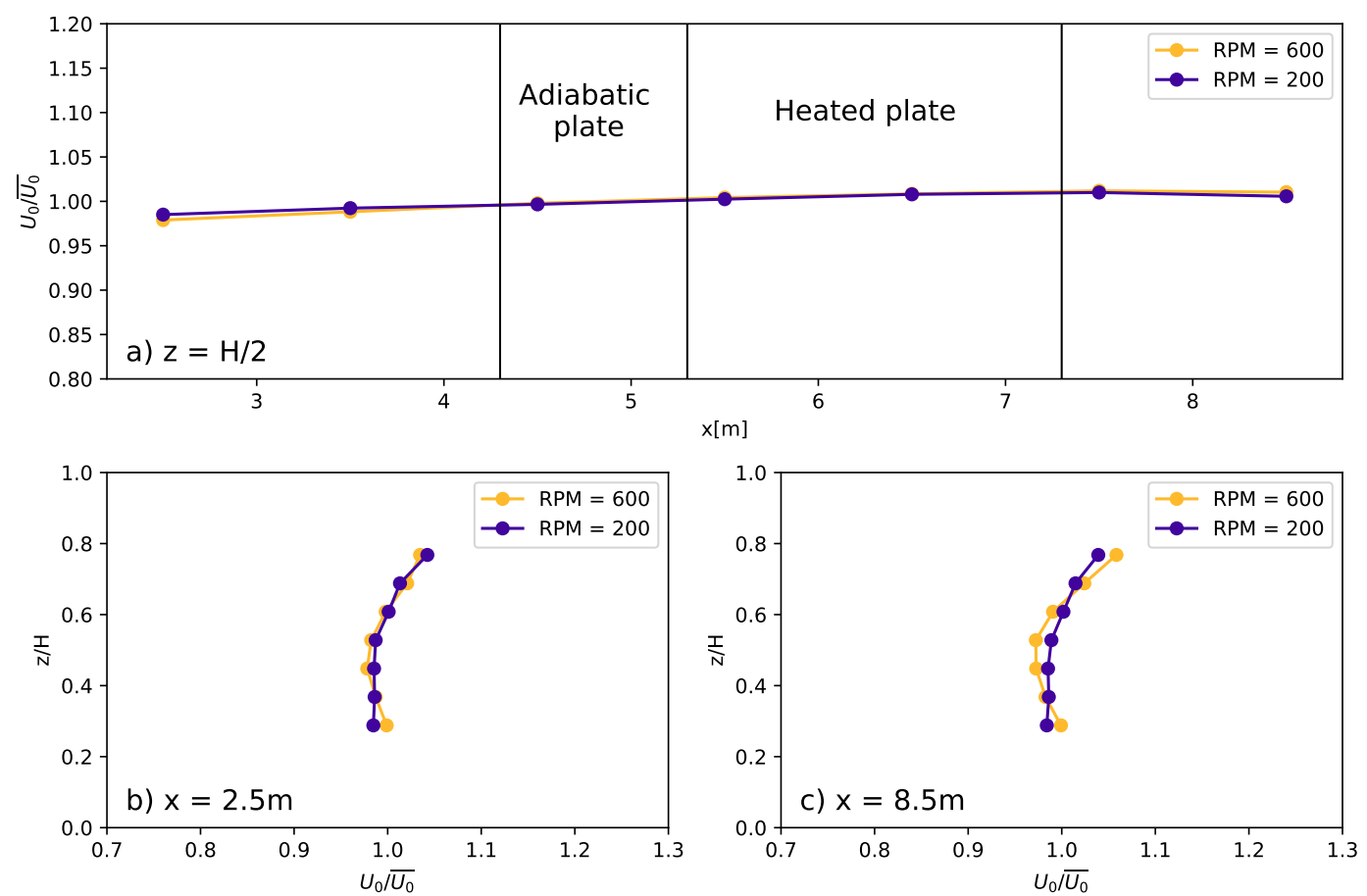

Figure 3.6: PWT velocity profile along the streamwise (a) and the wall-normal direction (b and c). 
time-average streamwise. A rotation rate of $600 \mathrm{RPM}$ corresponds to $\bar{U} \approx 5 \mathrm{~m} / \mathrm{s}$, and $200 \mathrm{RPM}$ does corresponds to $\bar{U} \approx 1.2 \mathrm{~m} / \mathrm{s}$.

We have measured velocity profiles in the Prandtl wind-tunnel for heights in a range between $z=0.4 m$ and $z=1 m$, outside the wind-tunnel boundary layer, our experiment is positioned in the bulk so that the free flow approximation at the leading edge hold valid. The maximum variation of the time-averaged streamwise velocity (along $\mathrm{z}$ ) is $8.6 \%$, and the maximum variation in the horizontal direction (along $\mathrm{x}$ ) is $3.2 \%$. The variation in pressure and therefore the velocity along the Prandtl wind-tunnel $\mathrm{x}$-axis is within the measuring error along a total measured length of $6 \mathrm{~m}$.

The free-stream turbulence $\theta=100 \cdot\left(U_{0}^{\prime} / U_{0}\right)$ at the hot-wire measurement point at different velocities is shown in the following table:

\begin{tabular}{||c|c||}
\hline$U_{0}[\mathrm{~m} / \mathrm{s}]$ & $\theta[\%]$ \\
\hline \hline 0.3 & 4.02 \\
\hline 1.2 & 3.67 \\
\hline 2.71 & 2.84 \\
\hline 4.48 & 2.61 \\
\hline 6.29 & 2.48 \\
\hline
\end{tabular}

\subsection{The heated plate}

A schematic of the heated section of the plate is shown in figure 3.7. The plate is $2 \mathrm{~m}$ long and $1 \mathrm{~m}$ wide. The plate consists of three layers glued together with a high thermal conductivity epoxy. These are:

1. Top layer: Anodized Aluminium - $25 \mathrm{~mm}$ thick

2. Middle layer: Polycarbonate $-5 \mathrm{~mm}$ thick

3. Bottom layer: Aluminium - $35 \mathrm{~mm}$ thick

The 3 layer design has already been used successfully in Rayleigh-Bénard convection cells [57] because it allows us to:

1. Generate a uniform surface temperature distribution in contact with the fluid. 


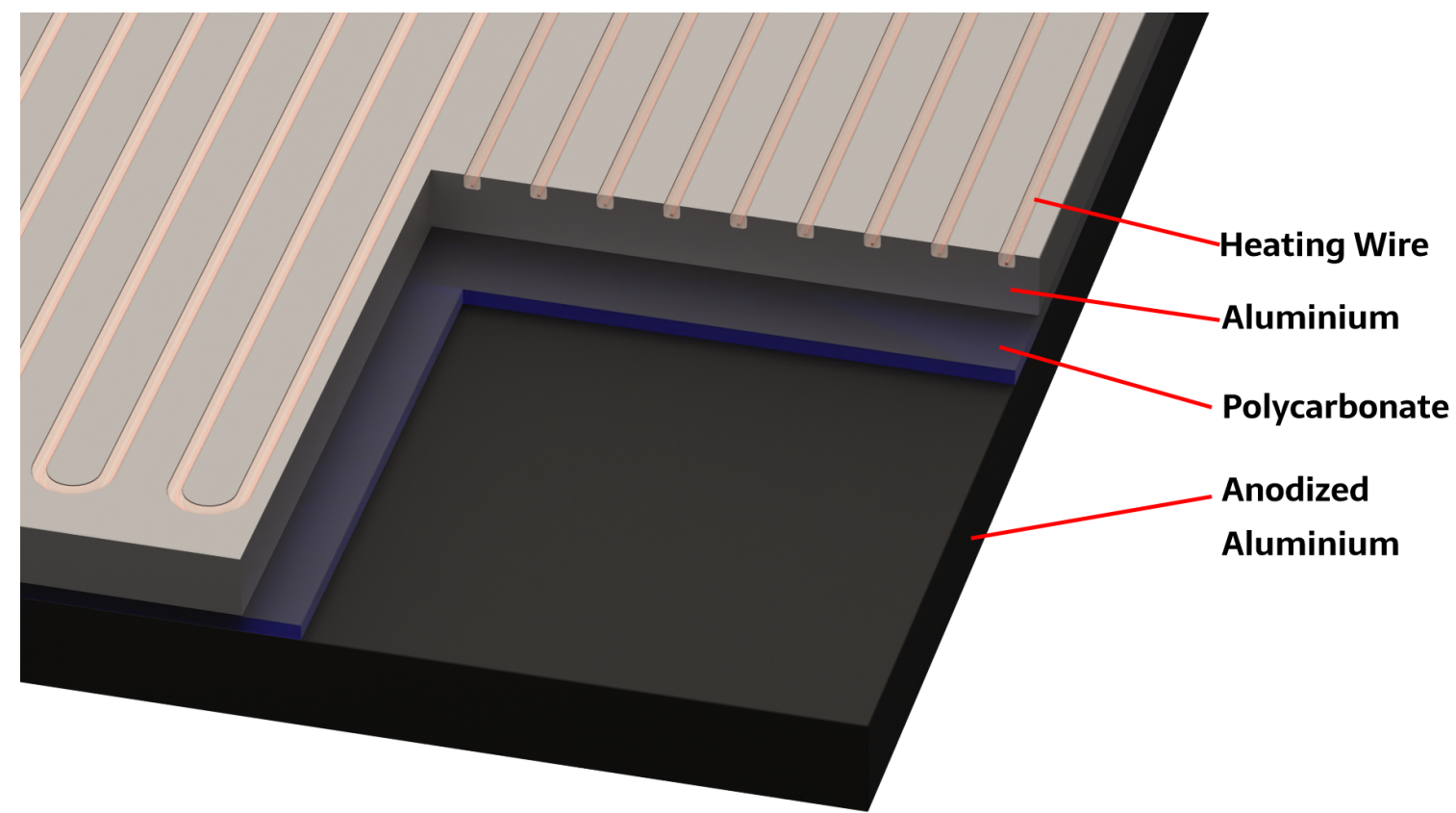

Figure 3.7: 3D Rendering bottom view of the heated plate. Slice shows layers composition.

2. A measurement of the local heat transfer at 21 different locations

3. Heat measurements without correction for heat loss through the bottom and the side possible.

This solution does allow us a spatially resolved heat transfer measurement. The low thermal conductivity of the poly-carbonate creates a larger gradient in the temperature between the bottom plate and the top plate, and therefore a higher precision measurement. Another benefit of this method is that it is a direct measurement of the heat flux between the plates, and therefore is not affected by heat loss to the bottom and the side. The aluminium plates are made of "Al Cast Plate G.AL" from GLEICH GmbH company. It is composed of "EN AW 7021" alloy. The declared surface roughness is $R_{a}<15 \mu \mathrm{m}$, thermal conductivity is $125<\lambda<155 \frac{W}{m K}$, full data-sheet can be found at gleich.de.

These 3 layers are glued together using a low viscosity and high thermal conductivity epoxy named "STYCAST 2850FT" that was degassed before it was applied. This special epoxy has been chosen to minimize the epoxy layer height and thermal resistance. In the bottom layer, is carved a $200 \mathrm{~m}$ long groove that host the copper heating wire fig. 3.7. The groove is $3 \mathrm{~mm}$ deep and $5 \mathrm{~mm}$ wide; the distance between the parallel lines of the groove is $15 \mathrm{~mm}$. This groove covers the entire bottom surface of the aluminium bottom 
plate and allows a uniform distribution of the heat. The copper wire is $200 \mathrm{~m}$ long and has a total resistance of $6.37 \Omega$ at ambient temperature. The copper wire is connected at its ends to a thick cable that has a very low resistance that is then used to connect the heating wire to the $2000 \mathrm{~W}$ DC power supply unit. As power supply we have chosen to use an Agilent, DC model '6675A', this unit can output 0-120V and 0-18A. The groove with the copper wire in position is filled with the same epoxy used to glue the plate's layers, it is used to hold the wire in position and for a better distribution of the heat from the wire to the bottom plate.

The bottom layer hosts an array of $40 \mathrm{~mm}$ long holes with a $7 \mathrm{~mm}$ diameter with a 35 degree angle of attack. Figure 3.8. The top layer also hosts an array of $40 \mathrm{~mm}$ long holes with a $7 \mathrm{~mm}$ diameter but with 45 degree angle of attack. The holes on the top and bottom layer are vertically coinciding with each other to measure the temperature drop across the polycarbonate plate. Thermistors are glued in position using the Stycast epoxy. To minimize heat loss, the plates are insulated at the sides by a $1 \mathrm{~cm}$ thick layer of insulation foam that has been applied to the left, the right and backside of the plate. The front side is insulated by being in contact with the "Adiabatic front plate" that will be discussed in chap 3.2.1.

\subsubsection{Thermistors}

We use in total 42 thermistors, (21 for each plate,) just to monitor the heat transfer through the heated plate, and one thermistor attached below the adiabatic plate to measure the incoming flow temperature. We use glass encapsulated semiconductor thermistors with a diameter of $2 \mathrm{~mm}$ and resistance around $100 \mathrm{k} \Omega$ (at ambient conditions) and a negative temperature coefficient to increase the measuring precision in our temperature range. The choice of high resistance is to minimize the relative contribution of the connecting wire to the measured resistance. A four-wire measurement was not necessary. The thermistors of choice are Tewa company - model "TT2-100KC3H-4". The thermistors have then been encapsulated in a hollow copper tube that has a $5 \mathrm{~mm}$ outer diameter and with $3 \mathrm{~mm}$ inner diameter. The copper tube encapsulation is used to facilitate the installation of the thermistors inside the plates. The copper tube does allow us to move the thermistors in position without breaking them, the copper also allows good thermal contact. 


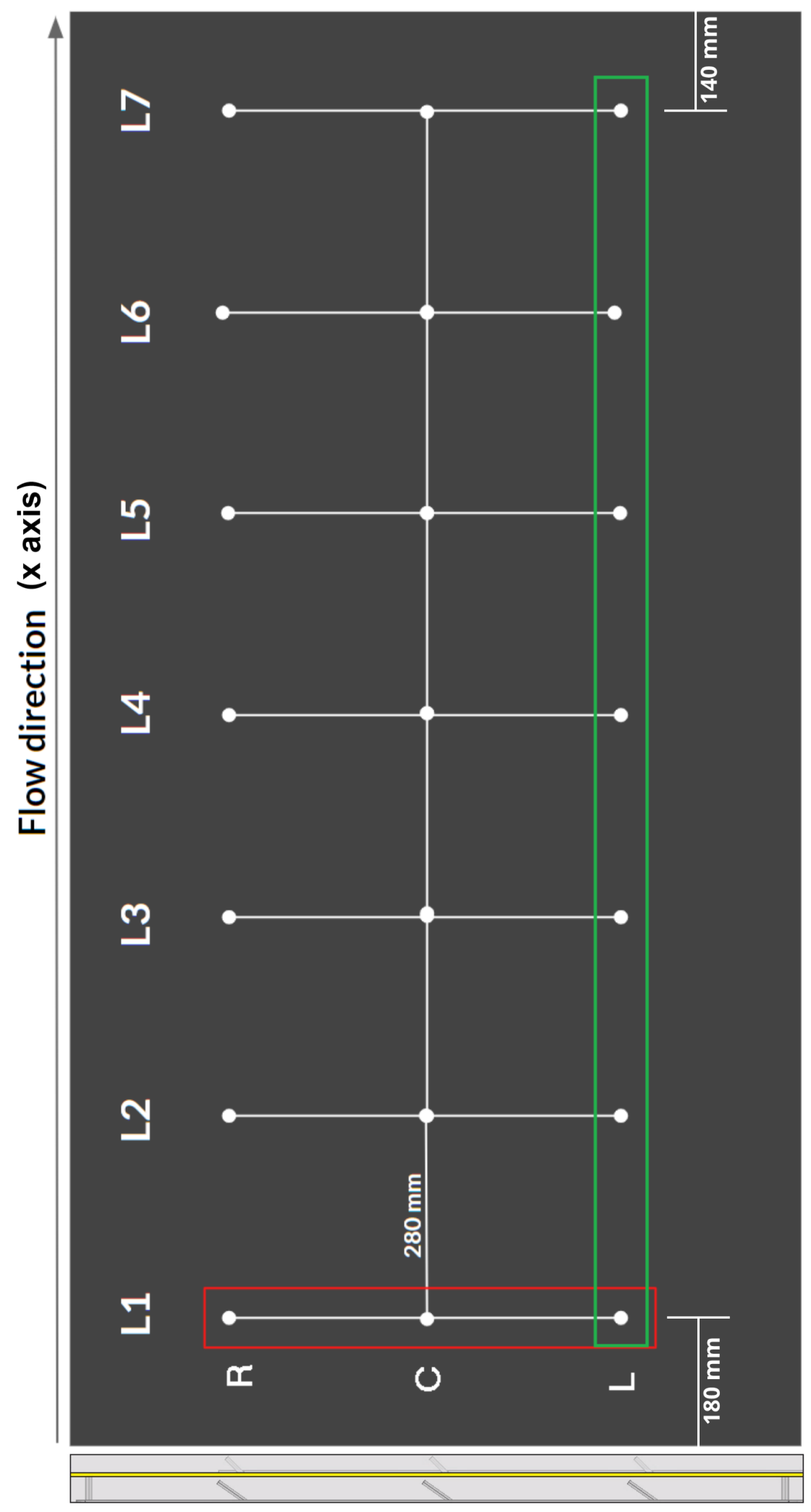

Figure 3.8: Top: Schematic top view, small circles represent the position where each thermistor is inside the plates. Bottom: Schematic front view, with inclined holes for the thermistors. 
All thermistors have been calibrated before being installed in position using a "Calibration box" that we have designed and that was built by the institute's machine shop. The thermistors were calibrated against a Lake Shore Cryotronics model "PT-103" as a reference thermistor. According to the Data Sheet offered by Lake Shore Cryotronics, the Pt-103 has a declared precision of $\pm 23 m K$ at $305 K$.

The resistance of the thermistors is measured with a Keysight data-logger model "34970A" with 3x Keysight boards model "34901A". Each board has 20 channels with a total of 60 available channels for one data-logger. A python program has been used to control the calibration process, regulating the temperature and acquiring the data from the datalogger. The thermal regulation has been done using a water bath circulator, (Thermo Scientific model "AC150",) this instrument can control the temperature of the water flowing with a stability of $\approx \pm 0.02 \mathrm{~K}$. The 45 thermistors are calibrated with a relative precision of less than $0.005 \mathrm{~K}$. After calibration, the thermistors have been placed inside the aluminium plates using conducting thermal paste to improve the heat transfer. The thermistors have been then fixed in position with low viscosity, high thermal conductivity epoxy. Furthermore, two thermistors have been positioned at the side of the hot-wire collecting temperature at the same height as the hot-wire.

After the assembly of the plate, the two thermistors labelled '2TR' and '4TC' were broken. Each thermistor is labelled with three characters that represent their position inside the plate. The first character is a number $\in\{1 \ldots 7\}$ which refers to the streamwise coordinate, where ' 1 ' is the first thermistor line and ' 7 ' the last thermistor line (green in fig. 3.8). The second character is either ' $\mathrm{T}$ ' or ' $\mathrm{B}$ ' and refers to the top and bottom plate the thermistor is placed in. The top plate is the plate of which the top surface is in contact with the flow, the bottom plate is the plate that is directly heated The third character represents the spanwise position of the thermistor and can by either Left $(\mathrm{L})$, Center (C) and Right (R) (highlighted in red in fig. 3.8).

\section{Support plate}

The heated plate is supported by a steel frame composed of $5 \times 2 \mathrm{~m} \times 0.03 \mathrm{~m} \times 0.005 \mathrm{~m}$ steel ${ }^{1}$ bars welded to $2 \mathrm{x} 1 \mathrm{~m} \times 0.03 \mathrm{~m} \times 0.005 \mathrm{~m}$ steel bars (schematic in fig. 3.9). All the steel bars are made out of 304 stainless steel with thermal conductivity $\lambda=16.2 \frac{\mathrm{W}}{\mathrm{mK}}$ with

\footnotetext{
${ }^{1}$ The use of steel has been necessary due to manufacturing and material strength constrains.
} 


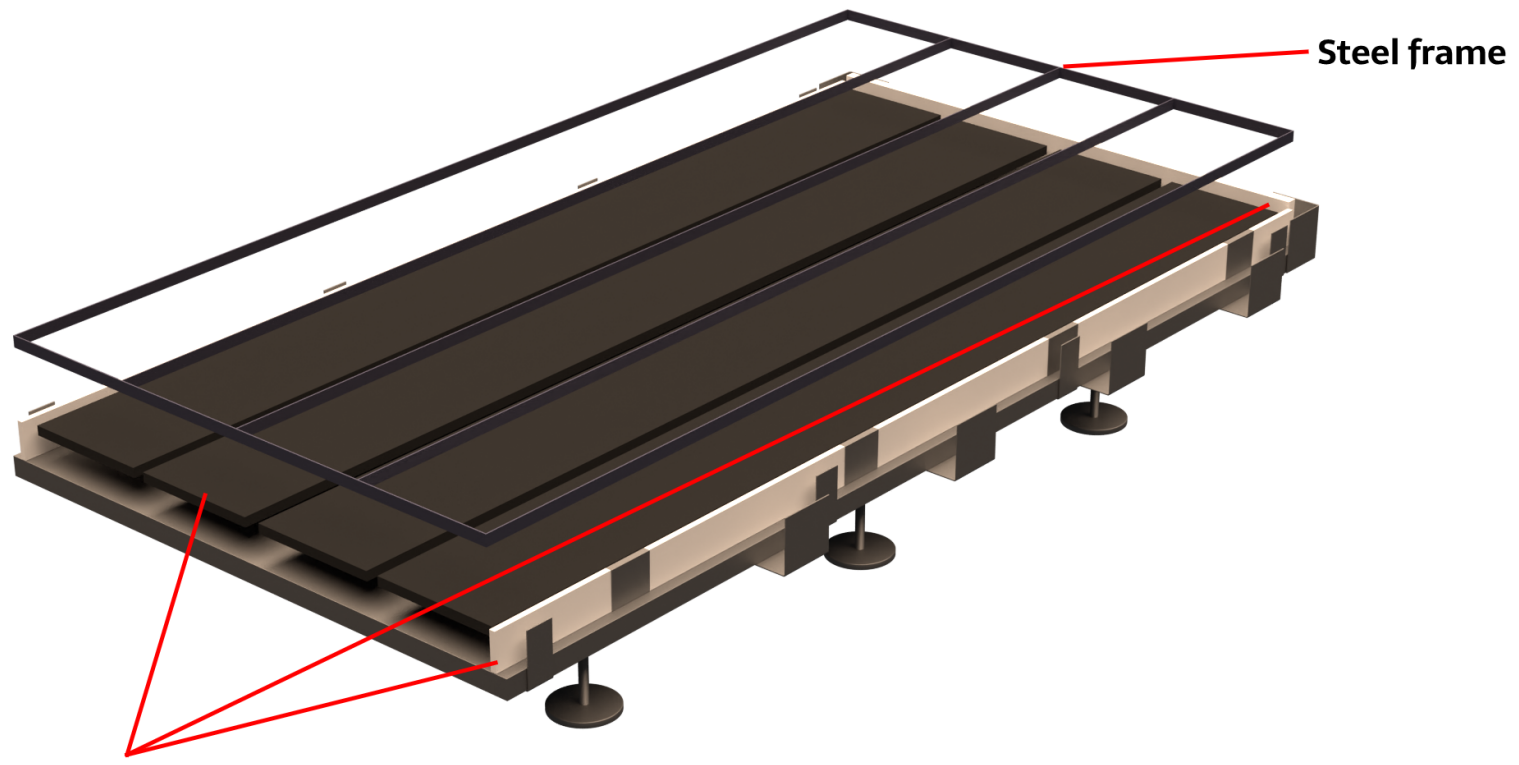

Thermal insulation

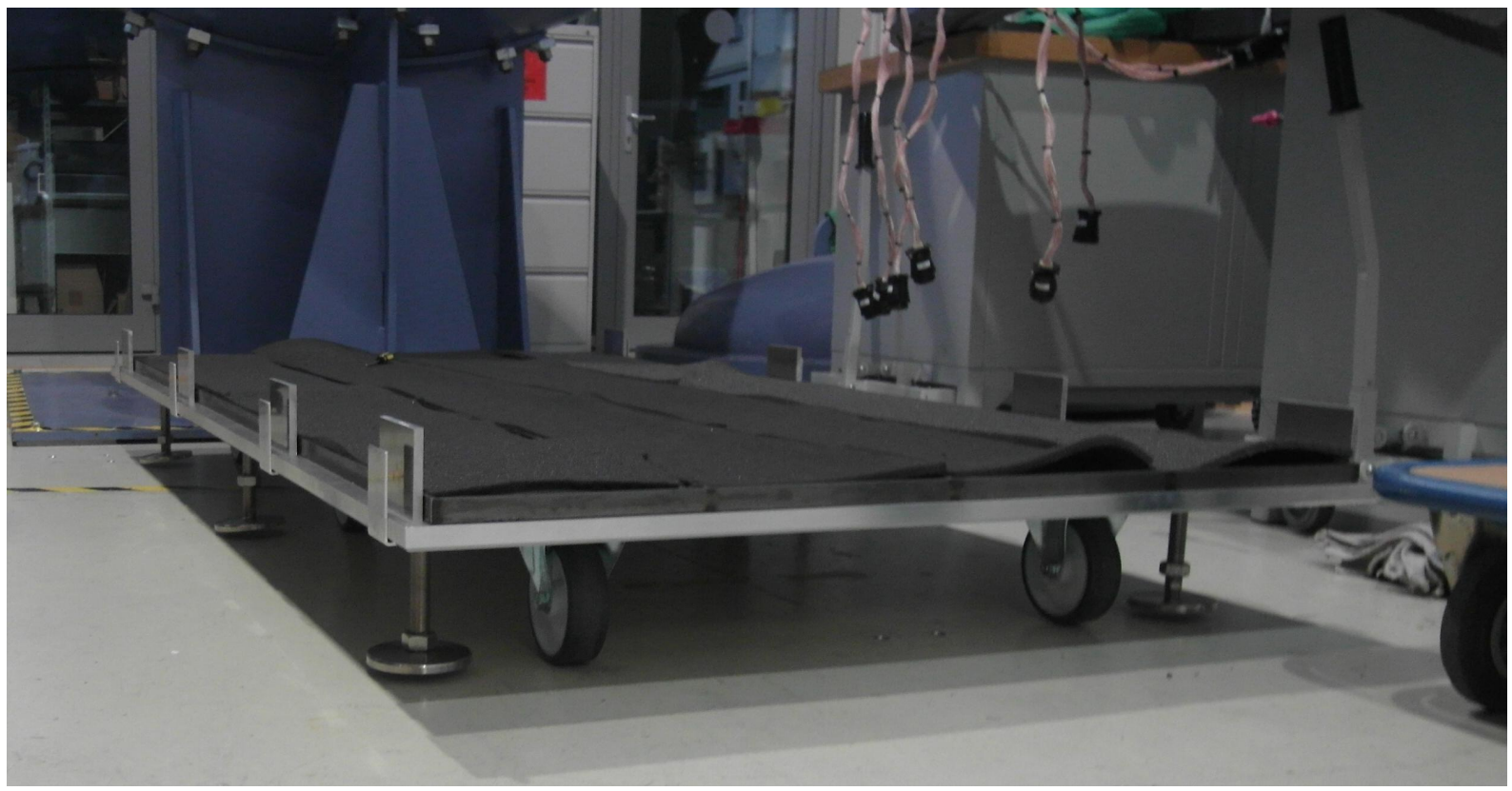

Figure 3.9: Top: 3D rendering, steel frame, support plate and insulation layers. Bottom: Base plate with insulation foam and steel frame. 
a total contact area between the support frame and the heated plate equal of $0.06 \mathrm{~m}^{2}$. It is important to minimize the contact to reduce the heat transfer through the support structure.

The steel frame is then connected and supported by a Steel base, made of 304 stainless steel $2,1 \mathrm{~m} \times 1,05 \mathrm{~m} \times 0,015 \mathrm{~m}$ (fig.3.9). To increase the thermal insulation the space inside the steel plate has been filled with thermally insulating foam (fig.3.9). The support structure with the heated plate on top sits on 6 legs which height can be varied.

We have also attached 6 wheels. With the legs retracted the plate is supported by the wheels and therefore can be easily moved inside the wind-tunnel. Once the measuring position is reached, the legs can be extended letting the feet support the plate. The height of the 6 feet can be adjusted independently. When in position the legs are used to level the surface perpendicular to the gravity.

\section{Front adiabatic plate}

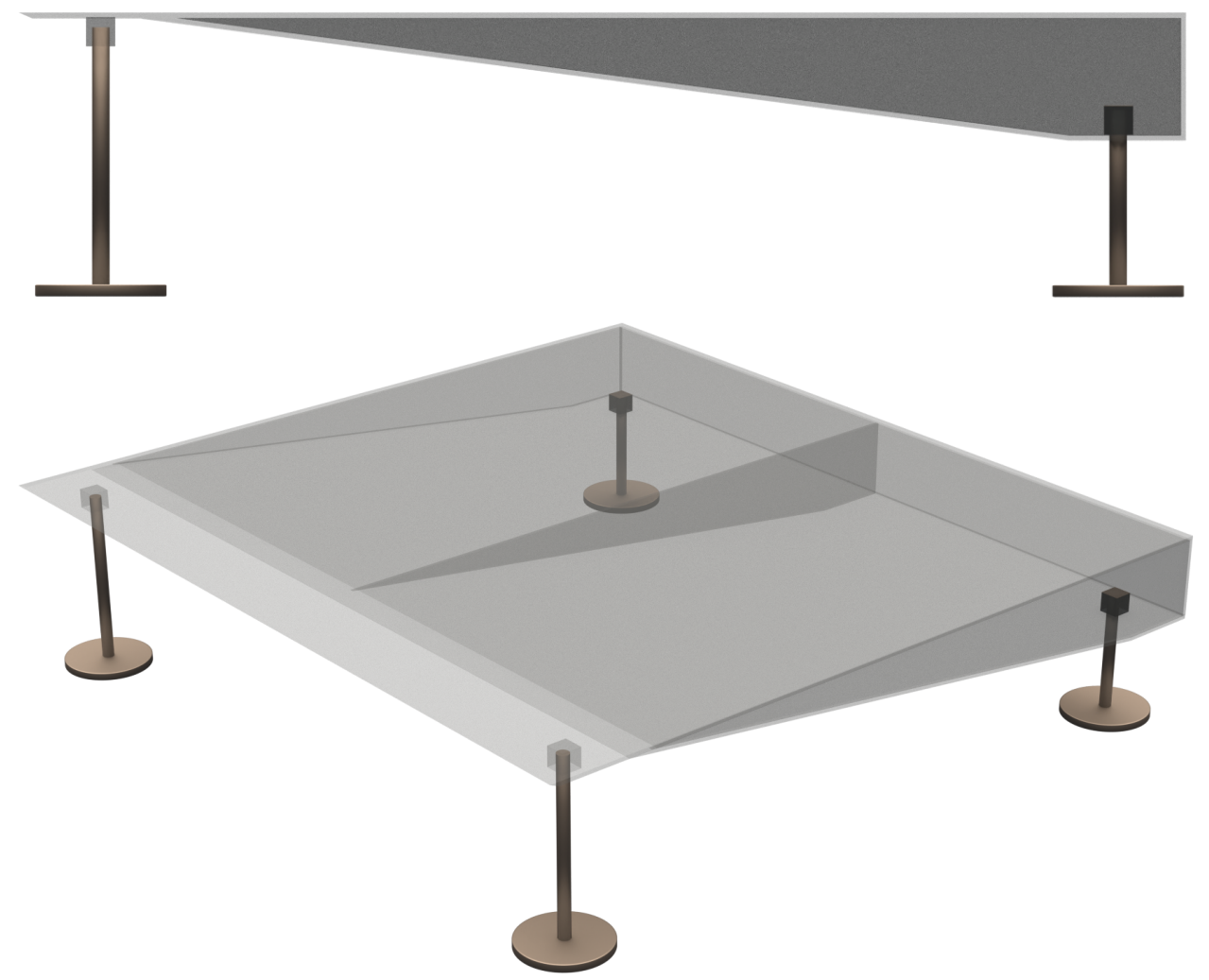

Figure 3.10: Schematic view, side and perspective view of adiabatic front plate

In front of the heated plate, another adiabatic plate was installed. This plate fulfils two purposes. It should reduce the flow separation at the interface between the free flow and 
the leading edge ${ }^{2}$ but it should also help to develop a boundary. The front plate ideally should not exchange any heat with the heated plate. It is also important to regulate the height of the front plate to have the most seamless transition possible between the front plate surface and the heated plate surface.

The front plate is a $1 \mathrm{~m} \times 1 \mathrm{~m}$ surface and is made out of $5 \mathrm{~mm}$ thick Plexiglas. The interface of the front plate with the incoming flow has a sharp edge with a $30^{\circ}$ angle and $10 \mathrm{~mm}$ length. This sharp edge should minimize the flow separation and hence keep the incoming flow laminar (fig. 3.10). The front plate is empty inside to minimize the heat exchange and reduce the cost. It is made of an assembly of cut Plexiglas sheets $5 \mathrm{~mm}$ thick, the sections are then glued together and it is equipped with $4 \mathrm{x}$ adjustable legs fig. 3.2.1.

\subsubsection{Heated plate temperature feedback loop}

In each plate, we have 21 thermistors and 1 thermistor is attached below the front plate to measure the incoming flow temperature $T_{0}$. One of the parameters that we control in our experiment is the top plate surface temperature. However, since we can set only the heat flux $q$ for the entire plate, but since we also want to account for small temperature variations of the incoming flow ${ }^{3}$, We decided to keep during an experimental run the temperature difference $\Delta T$ constant between the top plate temperature at the very first line and the incoming flow:

$$
\Delta T=\left(\frac{T_{1 T L}+T_{1 T C}+T_{1 T R}}{3}\right)-T_{0}
$$

To keep $\Delta T$ constant during an experimental run, we regulate the power input at the bottom plate $P\left(t_{i}\right)$ at each, equally spaced, measurement time $t_{i}$ using a proportionaldifferential feedback loop:

$$
P\left(t_{i}\right)=P\left(t_{i-1}\right)+\alpha\left(\Delta T_{\text {set }}-\Delta T\left(t_{i}\right)\right)+\beta\left(T_{B}\left(t_{i-1}\right)-T_{B}\left(t_{i}\right)\right)
$$

\footnotetext{
${ }^{2} \mathrm{~A}$ steep edge would have resulted in flow separation and therefore in the generation of eddies inside the boundary layer at the leading edge. Introducing those perturbations would result in reduced stability of the boundary layer.

${ }^{3}$ The lab temperature is only constant within $1.5 \mathrm{~K}$ or so.
} 
With $\alpha$ and $\beta$ being positive parameters that were optimized for lowest fluctuations of $\Delta T$ in time. $\Delta T_{\text {set }}$ is the desired $\Delta T$ that we want to keep constant for the experiment. $\Delta T\left(t_{i}\right)$ is the measured value at time $t_{i} . T_{b}\left(t_{i-1}\right)$ is the bottom plate average temperature in the previous time-step. $T_{b}$ current bottom plate average temperature.

\subsubsection{Heated plate constant heat transfer}

As pointed out already above, heat is introduced into the system via electric heating from a single long heating wire underneath the bottom plate. Hence, we assume that also the heat transfer from the surface of the top plate is rather uniform and spatially constant along with the plate. This can be seen in fig. 3.11, where we plot the local heat flux for the 7 different spatial positions along with the plate and 6 different $\Delta T$.

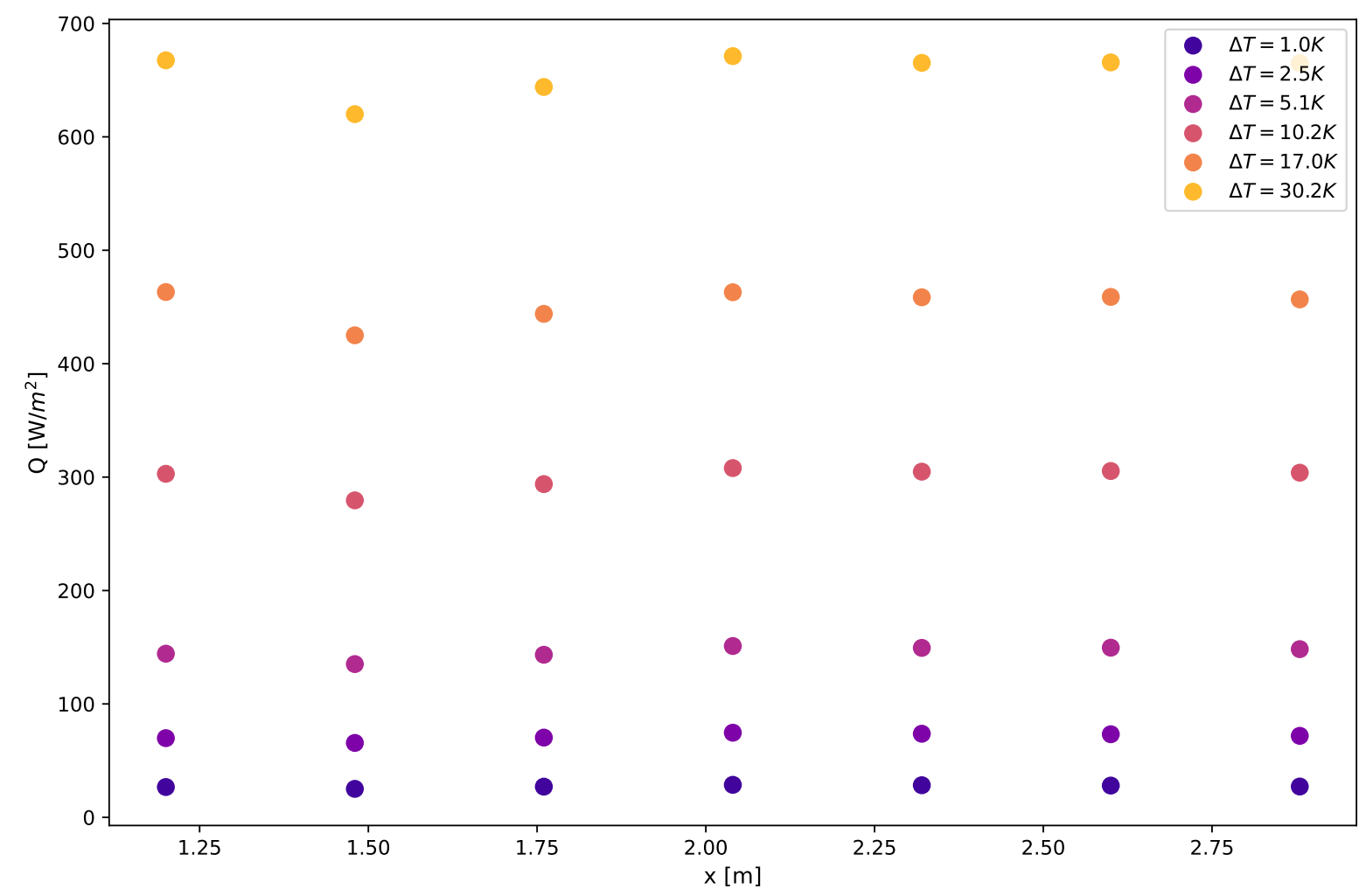

Figure 3.11: Local heat transfer along the different lines of thermistors.

The air that passes above the surface absorbs heat from the surface. The air becomes warmer proceeding along the $\mathrm{x}$-direction. Therefore, the constant heat transfer from the surface generates variations of the surface temperature of the top plate in the streamwise direction (the $\mathrm{x}$-axis) and is not constant anymore. It may also be useful to consider that 
the temperature gradient along the aluminium plate does drive heat transfer along with the metal. In our analysis, we neglect the effect of heat transfer through the plate. Correct for this effect in our analysis is not trivial. By knowing the amount of heat transported through the metal it is possible to reallocate it, this would result in a new thermal profile, but also an incorrect boundary layer evolution. The boundary layer evolution is a function of the temperature gradient it faces, and if we correct the temperature gradient, to obtain a proper correlation we need to correct also for the boundary layer. Unfortunately, the correlation between BL and temperature gradient is not trivial and at the most unknown, therefore we have chosen to neglect this effect.

\subsection{Constant Temperature Anemometry (CTA)}

To measure the flow velocity, we use Constant Temperature Anemometry. The constant temperature anemometry is coupled with hot-wires. A hot-wire is a fork structure that holds a wire that is heated through an electric current. A CTA feedback loop keeps the temperature of that wire constant at around $280^{\circ} \mathrm{C}$ by adjusting the power input fig. 3.13.

When a hot-wire is immersed in a moving fluid the electrical power necessary to keep its temperature stays constant changes proportionally to the heat transfer from the wire to the colder fluid. The heat transfer in turn is a function of the fluid properties (like humidity and density) and the velocity of the fluid. Therefore by measuring the power input, we can derive the velocity of the fluid, assuming that all the relevant fluid properties stay constant in time.

Hot-wires are the best compromise that allows us to measure in the range of velocity we are interested in, and with a high temporal resolution. Unfortunately with the hot-wires one can only conduct localised measurements at a single point in space.

\subsubsection{Hot-wire probes}

In our experiment, we have chosen to use a hot-wire probe that has been specifically designed for boundary layer measurements. (Dantec Hot-wires, model: "55P15", see fig. 3.13) mounted on a Dantec Probe Support that was $235 \mathrm{~mm}$ long. These hot-wire probes are designed for measurements that are close to a surface. Their body is designed to 
minimize its interaction with the airflow. The wire diameter is $5 \mu \mathrm{m}$. More specifications can be found at the Dantec official web-page [58].

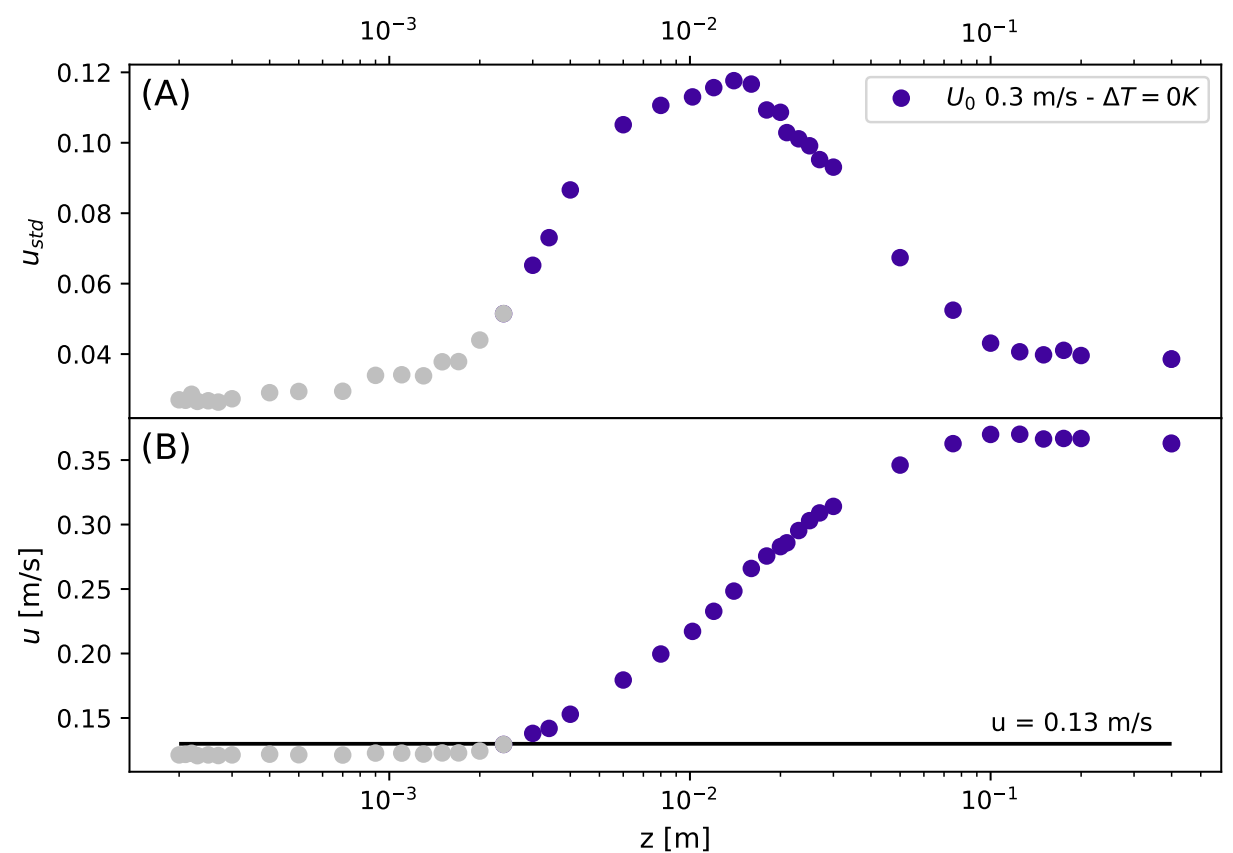

Figure 3.12: Velocity data collected at extremely low velocity. Velocity standard deviation (A) and time averaged-velocity $(B)$ as a function of the vertical distance from the plate surface. Average velocities $0.13 \mathrm{~m} / \mathrm{s}$ (grey triangles) were not used for further analysis.

The hot-wire data are collected with a Dantec " 4 Channel MultiCTA" (model "54N82"). The MultiCTA is responsible for the control of the hot-wire's temperature feedback loop. The output of the CTA is the voltage that is necessary to keep the hot-wire at a constant temperature. The MultiCTA can output an analogue signal with frequencies up to $20 \mathrm{kHz}$. That signal is then read with a digital analogue converter (Labjack model "T7 Pro") and converted into a 12bit digital signal using the stream mode (used in our experiment).

Due to the "hot" nature of the CTA, it has some limitations. The Dantec 55P15 that we use for this experiment can measure velocities as low as $0.05 \mathrm{~m} / \mathrm{s}$, but the influence of the natural convection is relevant up to $0.2 \mathrm{~m} / \mathrm{s}$. The thermal plumes that may detach from the hot-wire do translate into a spike in thermal transfer and therefore in the measured velocity. The time average does allow to minimize the contribution of these spikes in the total measurements. In our experiment, we have excluded all the measurements below $0.13 \mathrm{~m} / \mathrm{s}$ because they resulted unreliable in our tests as shown in fig. 3.12. It is 


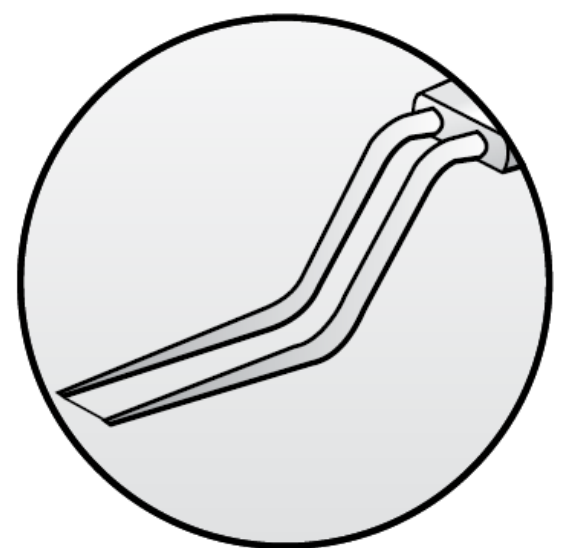

$55 \mathrm{P} 15$

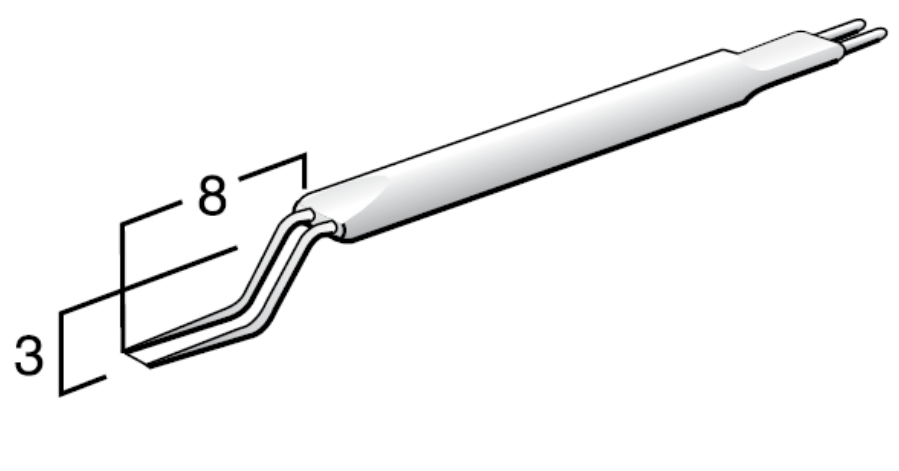

Boundary layer type

Figure 3.13: Schematic of the boundary layer hot-wire used in our experiment (Dantec 55P15) Image from [59].

expected at the surface proximity to observe the velocity drop to 0 , but observe a constant value of velocity, this is due to the hot-wire natural convection becoming dominant.

There are then some factors that we have to consider if we want to use hot-wires for our measures. In our setup, the fluid temperature changes around the hot-wire, when we get closer to the heated surface. According to the Dantec Dynamics web-site, [60] the correction for the temperature variation can be written as:

$$
E_{\text {corr }}=E\left(\frac{T_{w}-T_{*}}{T_{w}-T_{0}}\right)^{1 / 2},
$$

with $T_{W}$ being the hot-wire temperature, $T_{0}$ the fluid temperature, $T_{*}$ the ambient reference temperature, $E$ the Voltage, and $E_{\text {corr }}$ the corrected Voltage.

Hot-wires are very sensitive and might show s slow drift over time. For this reason, it is required to calibrate them often. Hot-wire probes can be calibrated either with a jet of a known velocity or in a flow against a pitot tube. To calibrate the fast Dantec CTA probe we used a time-averaged pre-calibrated hot-wire anemometer, a Testo model "405i". This hot-wire is glass encapsulated and has a slow response time. The instrument measures velocities with a resolution of $0.01 \mathrm{~m} / \mathrm{s}$, and accuracy of $0.1 \mathrm{~m} / \mathrm{s}$ [61].

For the hot-wire calibration the hot-wire probes were positions in the center of the wind-tunnel (40 cm above the plate) at about the same $\mathrm{x}$ position at which later mea- 
surements were taken. The Testo "405i" was mounted in between both CTA probes. The calibration of the CTA covers a range of velocities between $0.1 \mathrm{~m} / \mathrm{s}$ and $7 \mathrm{~m} / \mathrm{s}$, in case of higher speed measures a specific calibration is done that reach a maximum velocity of $12 \mathrm{~m} / \mathrm{s}$. The hot-wire data are collected at $10 \mathrm{kHz}$ for 2 minutes, the average voltage of these two-minute measures are then used to fit the King's Law curve [62] [63] [64] against the velocity measured with the Testo "405i".

\section{Important note:}

It is extremely important to address a question that may arise when we will present the data collected at different surface temperatures. The increase in the surface temperature does rise the flow temperature. As explained in this chapter the hot-wire measures the velocity by measuring the heat that is transferred from the hot-wire to the flow. By heating the flow we are changing the temperature difference between the hot-wire and the flow, therefore the measured velocity may be affected, resulting in a seemingly lower measured velocity.

Using formula 3.3 we can calculate the relative error in the measurement due to our temperature shift. The hot-wire temperature is during the calibration at a temperature delta of $+250 \mathrm{~K}$ from the flow. The maximum flow temperature increase we have measured at the wall proximity is $8 K$, resulting in a maximum error of $1 \%$. Therefore is possible to exclude any relevant effect in the measured velocities due to heat and the correction can be neglected.

\subsubsection{Height control system and probe holder}

Given the small diameter of the hot-wire of $5 \mu \mathrm{m}$ and length of $1.25 \mathrm{~mm}$, we can measure the velocity with very high spatial and temporal resolution and very close to the surface. For that reason, the 2 parallel hot-wires are mounted on a twin arm system that let us control the height with a precision of up to $100 \mu \mathrm{m}$. We note that in most of our experiments, the boundary layer was just a few centimeters high, with the largest velocity gradients in the viscous sub-layer that was about half a millimeter thick for the largest Reynolds number.

In figure 3.14 we see the rail system installed on wind-tunnel floor. The rails support and allow movement of a 2 axis movement system controlled by an ISEL Step motor- 


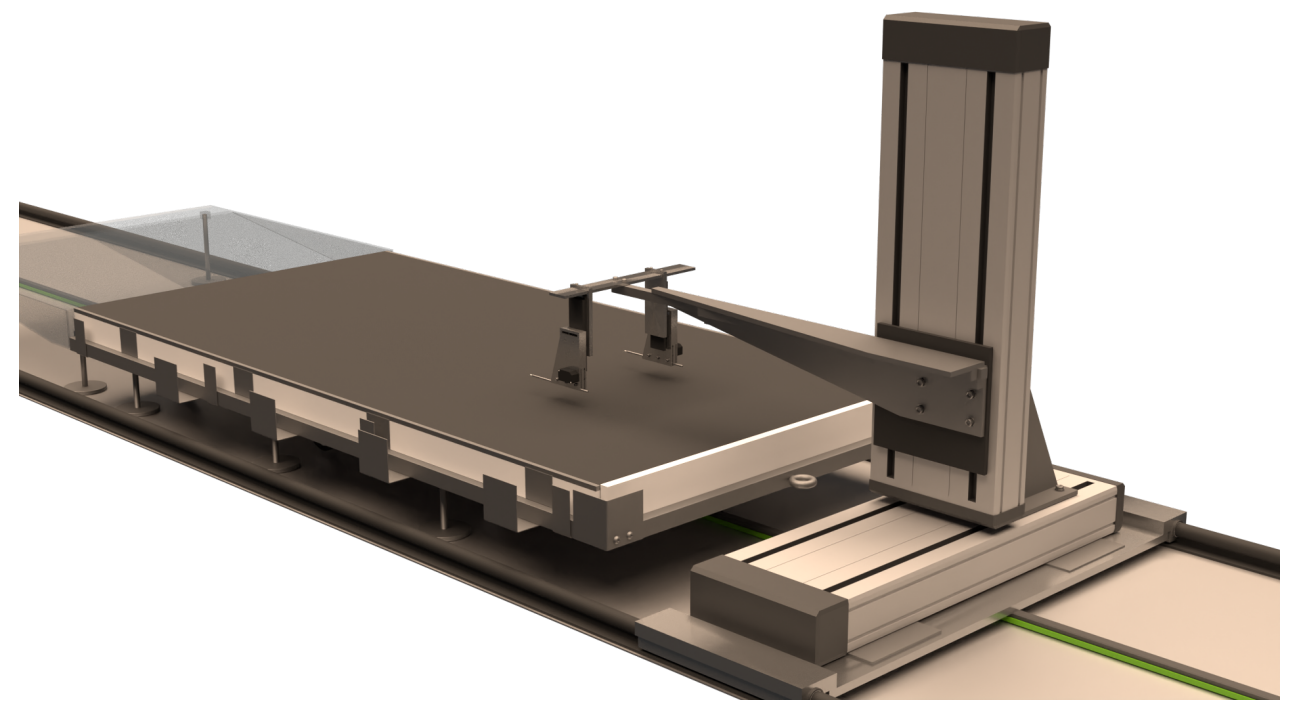

Figure 3.14: 3D rendering two-axis moving stage, extensible arm, and hot-wire control systems

Controller model "C142-4". The system allows the precise positioning of our sensors in the $\mathrm{x}, \mathrm{y}$, and $\mathrm{z}$ direction and lets us move at a height between $0.4 \mathrm{~m}$ and $1.4 \mathrm{~m}$ from the bottom of the wind-tunnel (z-axis), and between $-0.5 \mathrm{~m}$ and $0.5 \mathrm{~m}$ on the Y-axis with 0 being the center of the wind-tunnel. The 2 axis system has a $1 \mathrm{~mm}$ precision. Along the $\mathrm{x}$-direction we can move the system up to $6 \mathrm{~m}$ into the test section of the wind-tunnel. The hot-wire probe holders are mounted on an arm that is attached to the 2 axis movement system. The arm has been designed by us for this experiment. It can be extended up to 2 meters and allow us if needed to measure along the entire plate length.

Due to the thermal expansion of the plate, an increase in its temperature results in an increased plate thickness. This change is not negligible in our experiment. To achieve a high level of precision and repeatability of the measurement a micrometre precision step motor has been coupled with a high precision laser sensor for precise vertical positioning of the hot-wire probes.

We used a two Thorslab MTS model "MTS50/M" (from now MTS) as step/step motor and two Waycon Lasersensor (from now LS) model "LAR 10 5V" as distance measuring sensor. The coupling of the MTS and LS does allow us to increase the positional awareness precision of the setup up to $\pm 10 \mu m$. The LS is powered with a Keysight 200W DC Power Supply model "E3634A", and the output is read through the Labjack DAC T7 Pro that is already used to read the Hot-wire output.

The experimental setup has 3 different grades of precision depending on the distance 


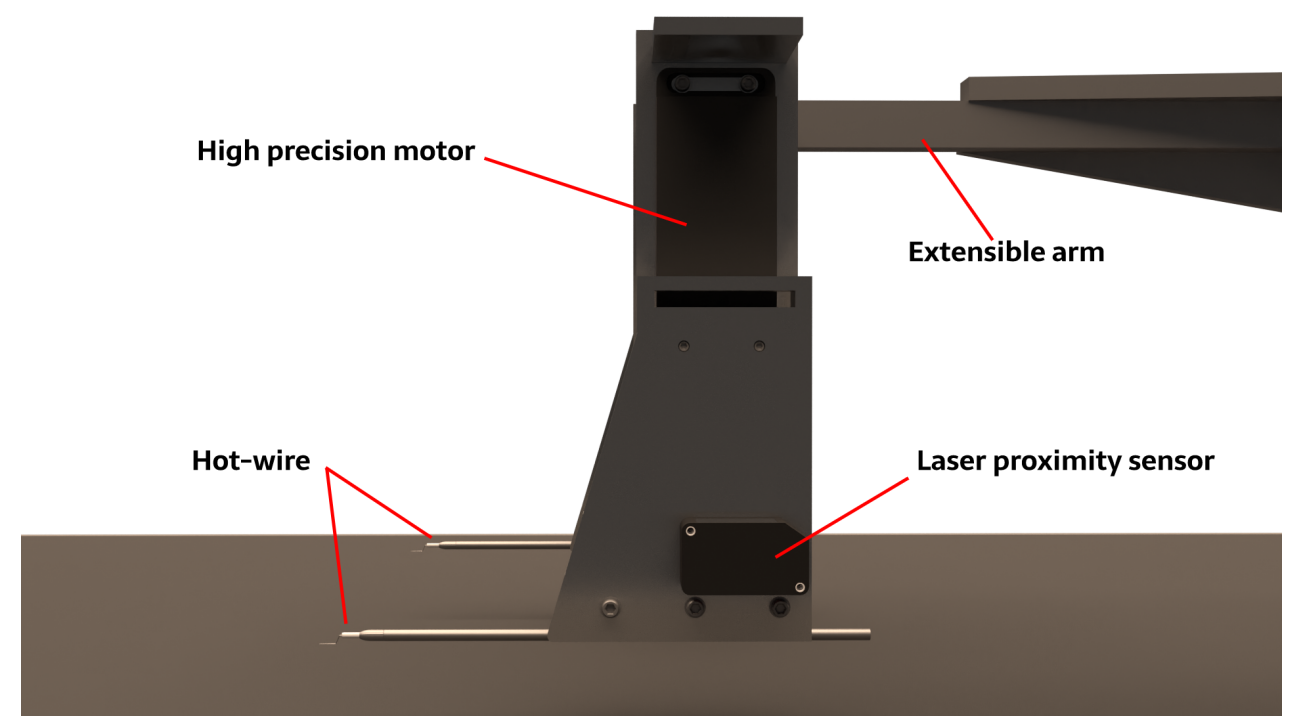

Figure 3.15: 3D rendering, side view of hot-wire support system

from the surface:

- 0 to $5 \mathrm{~mm}$ is the operative range of the LS, where the probe can be set with very high relative precision. Setting the probe to the correct position is computer programcontrolled.

- 0 to $30 \mathrm{~mm}$ is the operative range of the MTS. The MTS has an internal system that let it move with very good precision. Computer program-controlled.

- In the range from 30 to $400 \mathrm{~mm}$ we are out of the operative range of high precision systems. In this range, we use BMTS to move the arm along the $\mathrm{z}$ position. This system has a precision of around $1 \mathrm{~mm}$ and is controlled manually.

\subsection{Trip wire}

During our tests, we have installed a tripwire trying to trigger the fully turbulent boundary layer already at lower velocities. Measurements with and without a tripwire are compared in fig. 3.16. Note, that the measurement there have been normalized by the bulk velocity. Purple data are collected at $U \approx 0.35 \mathrm{~m} / \mathrm{s}$ in what we will introduce later as a transitional regime; Orange data have been collected at $U \approx 2.5 \mathrm{~m} / \mathrm{s}$, in what we will define later as turbulent boundary layer. We have tested two different configurations, one with a $1 \mathrm{~mm}$ thick tripwire that was placed $x=10 \mathrm{~cm}$ away from the leading edge of the adiabatic plate. 
The comparison between the measurement done with and without a tripwire showed no significant difference for the Reynolds number in our measurements.

The position of the tripwire was too close to the boundary layer stable region, therefore the perturbations generated is dissipated by the viscosity. The tripwire presence has shown to not affect the measures and we will not specify if a measurement has been done with or without tripwire. In fig. 3.16 we show that despite the presence of the hot-wire, we have no noticeable difference in the boundary layer velocity profile.

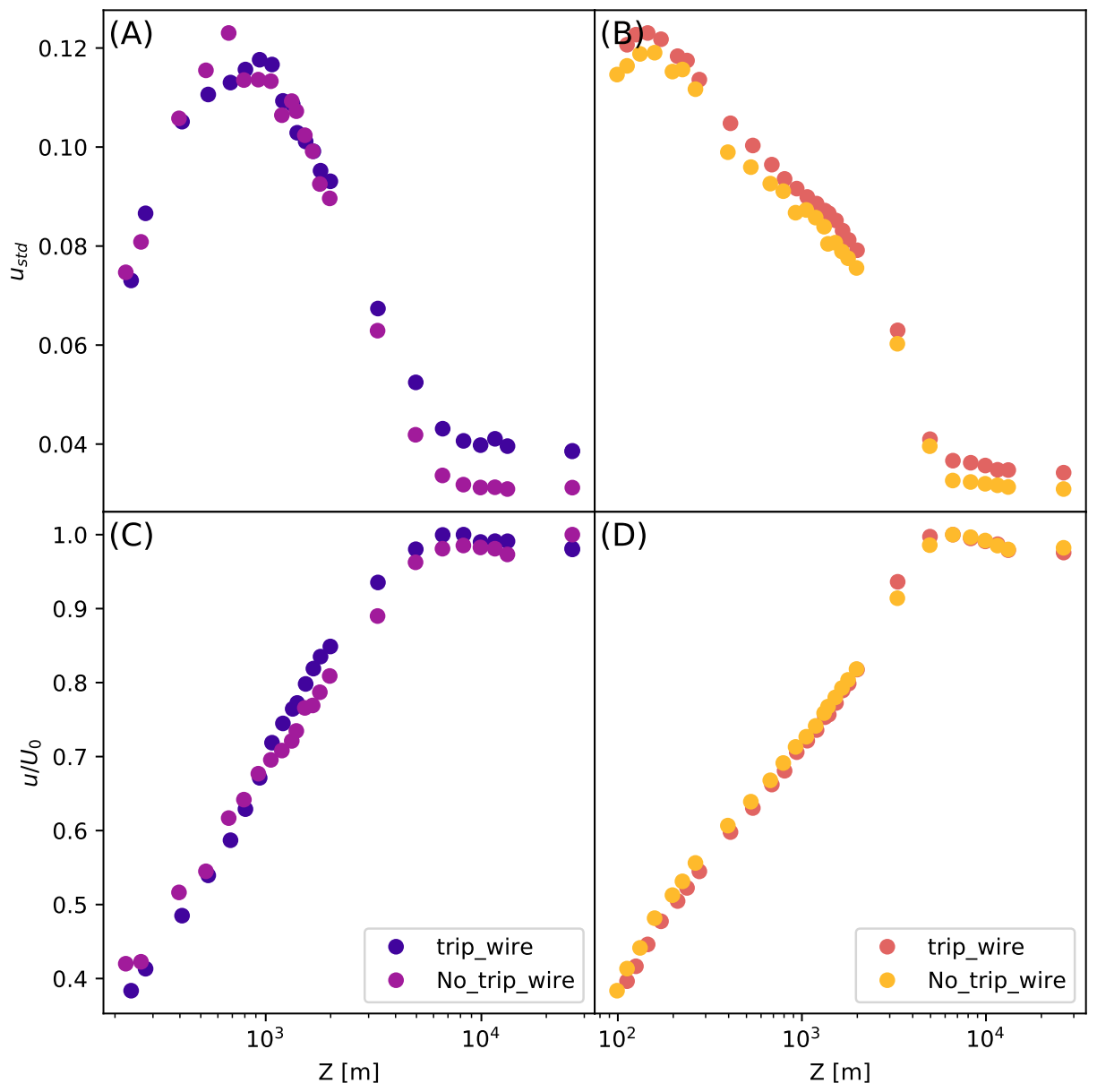

Figure 3.16: Velocity fluctuations $(A$ and $B)$ and time averaged streamwise velocity $(C$ and $D)$ as function of the vertical distance from the plate. (A) and $(C)$ data collected with $U_{0} \approx 0.3 \mathrm{~m} / \mathrm{s}$, (B) and (D) data collected with $U_{0} \approx 2.5 \mathrm{~m} / \mathrm{s}$.

\subsection{Vertical thermistor bar}

Initially, we attempted to measure the temperature profile in the boundary layer using two thermistors that were mounted close to the hot-wire probes. However, due to the slow 


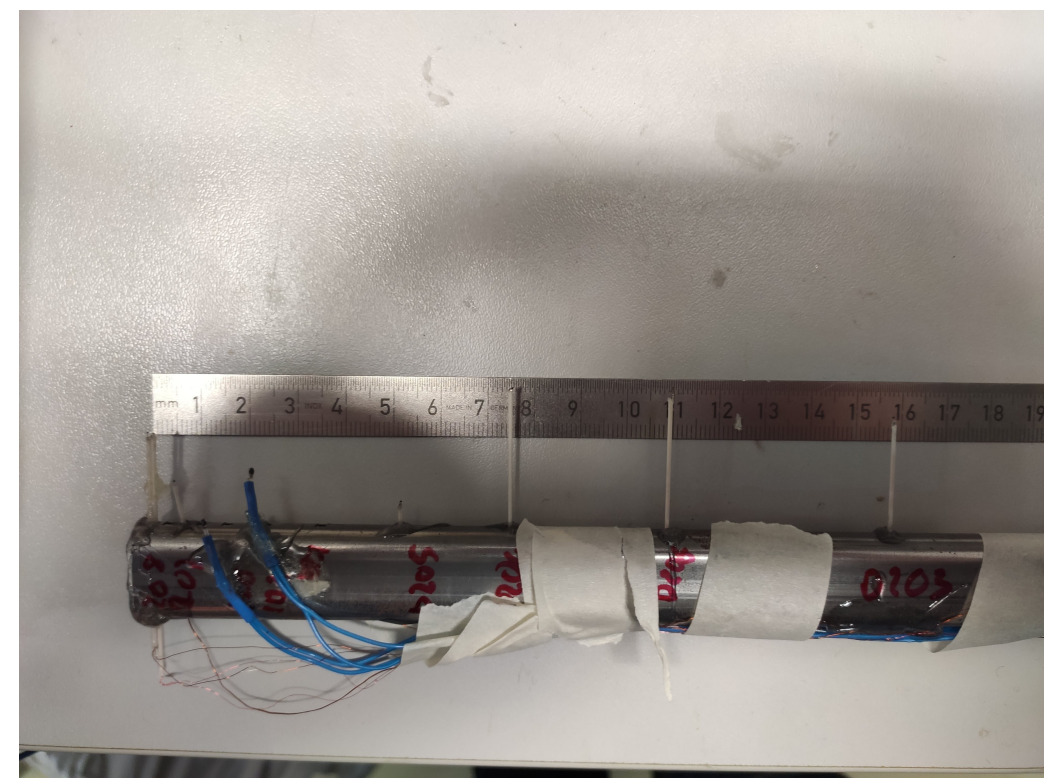

Figure 3.17: Photo of the thermistor rod used in vertical profile temperature measurement

response time of the thermistor, measurement times necessary to reach good statistics were very large and hence measurements became unfeasible long.

Instead, we have mounted 12 thermistors at a different height along a rod so that they could measure the temperature at their positions almost simultaneously. We used a set of standard thermistors with parameters similar to what has already presented in section 3.2.1. We used a mix of $2 \mathrm{~mm}$ glass encapsulated thermistors (to measure far from the surface,) and $0.5 \mathrm{~mm}$ non encapsulated ones (to measure close to the surface,) that were available at the institute.

Although our thermistors are small and their response time close to 1 second, they are too slow to measure fast velocity fluctuations inside the boundary layer. The calibration procedure has been done as explained in section 3.2.1, the smaller thermistors because of their fragility has not been placed inside the aluminium holes, but instead taped on top of it. The final calibration due to lack of time has been done with the calibration box but without the necessary optimizations for thermal contact. The calibrated thermistors have then been attached to the rod using hot glue at the positions on the $\mathrm{z}$-axis in mm: $2,3,7,12,22,75,155,317,455,652,935,1100$. The rod is then placed vertically on top of the heated surface and kept in position with the help of a connector attached to the PWT ceiling. 


\subsection{High speed camera setup}

Visualization of the flow structure is often helpful to better understand the state and dynamics of the flow. For that reason, we used a high-speed camera (Vision Research, Phantom Camera model "VEO 4K-L990"), with a resolution of up to 4096x1440 px and a maximal frame-rate of $1200 \mathrm{fps}$. The camera has been equipped with a "Nikon Lens Nikkor $28 \mathrm{~mm} \mathrm{f} / 2.8 \mathrm{AF}-\mathrm{D}$ ". The region of interest we were looking at was $88 \mathrm{~cm}$ long and reached from the plate to the bulk region of the flow.

For illumination, we used a 7 Watt green LED. The light passes through an optical fibre to a semi-cylindrical lens that converts the beam into a $2 \mathrm{~d}$ light sheet. The light intensity is not perfectly uniform along the measured volume, this is because we are not using a laser sheet. The sheet is then positioned to illuminate a portion of the incoming flow with a direct beam perpendicular to the camera along the line in the fig.3.18.

The camera was mounted on a rail system so it could be moved along the PWT on the side of the heated plate (fig. 3.18). The camera is connected to a Windows 7 laptop through a $1 \mathrm{~Gb}$ Ethernet connection and controlled via the program provided by Vision Research named PCC version 3.1.772.0.

To visualize the flow, we used an industrial fog machine from the JEM model "ZR44 High Mass" coupled with Pro Smoke Super liquid. The fog machine is seeding the flow with vaporized droplets before the propellers. The fog is then passing through the propeller, the laminarizing grid and then over the plate. The fog is composed of warm glycerol particles, therefore they tend to raise in the flow creating a gradient of distribution of particles with a higher density close to the ceiling of the wind-tunnel.

\subsection{Experimental procedure}

The experimental procedure depends on the data that were acquired. In this section, we will show and explain these procedures and how data were acquired and processed. 


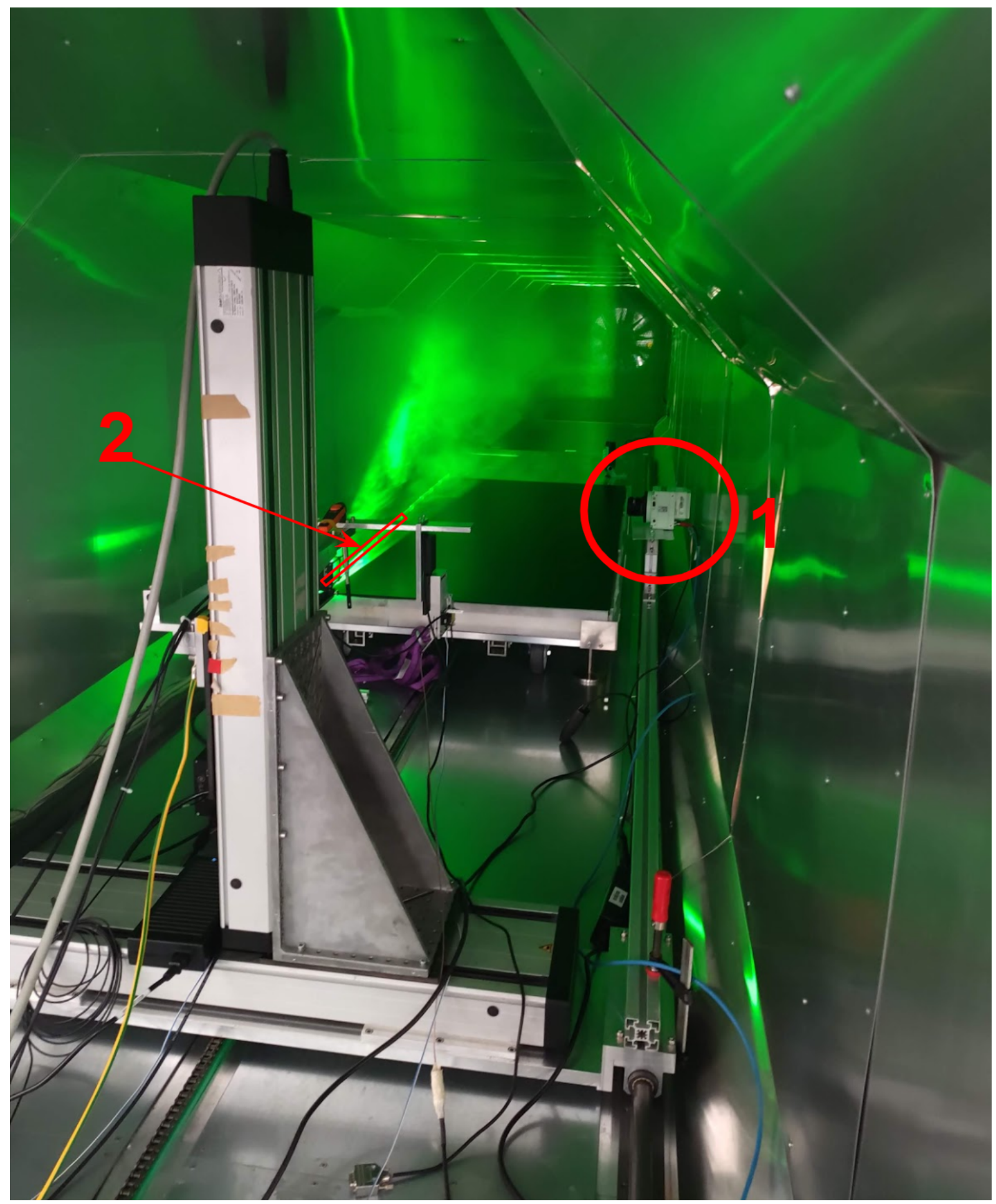

Figure 3.18: 1. Camera mounting position - 2. Visualized layer. 


\subsubsection{Heat transfer measurement}

The resistance of all thermistors as well as the voltage drop across the heating wire was measured with the Keysight multimeter and transferred to the attached computer (Mac OS X). The computer collects these data from the multimeter but also receives from the power supply the value of the current that is applied to the heating wire. The temperature control feedback loop is executed by the Mac computer that then corrects the power output of the Power supply unit (fig. 3.19).

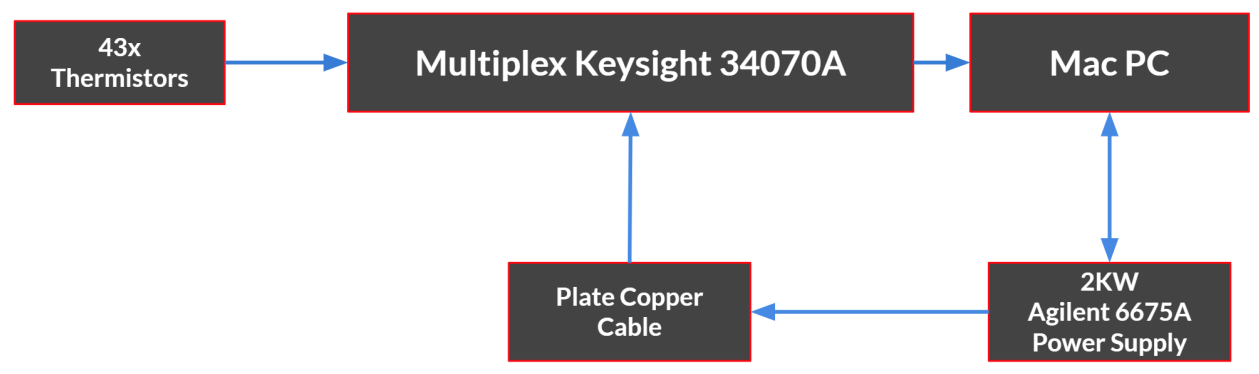

Figure 3.19: Schematic of connections layout for the heat transfer measurement setup

Once the PWT wind speed is set we activate the heated plate temperature regulation loop. It is necessary to wait a certain amount of time before the real measurement can start. The thermal inertia of the plate requires a few hours to equilibrate. This time may vary according to the measurement parameters we set. A typical measurement lasts for about one day to acquire enough data to get good statistics

\subsubsection{Temp controlled hot-wire velocity measurement}

The temperature control feedback loop is the same as in section 3.7.1. In this setup, we have also an exchange of data between the Mac computer and a Windows PC that is necessary to control the vertical position of the hot-wire probes.

A python script is responsible for the height regulation. The program moves the 2 Thorlabs MTS at the operative range for the laser sensors. The laser sensor output is read through the DAC. A controlled feedback loop moves the hot-wire probes according to the laser read position to the desired position. The position $z_{0}$ is the lowest position for our measurements. It is obtained by manually calibrating the hot-wires position against a sheet of paper, using light to cast a shadow and in this way to minimize its distance to the surface without touching it. 


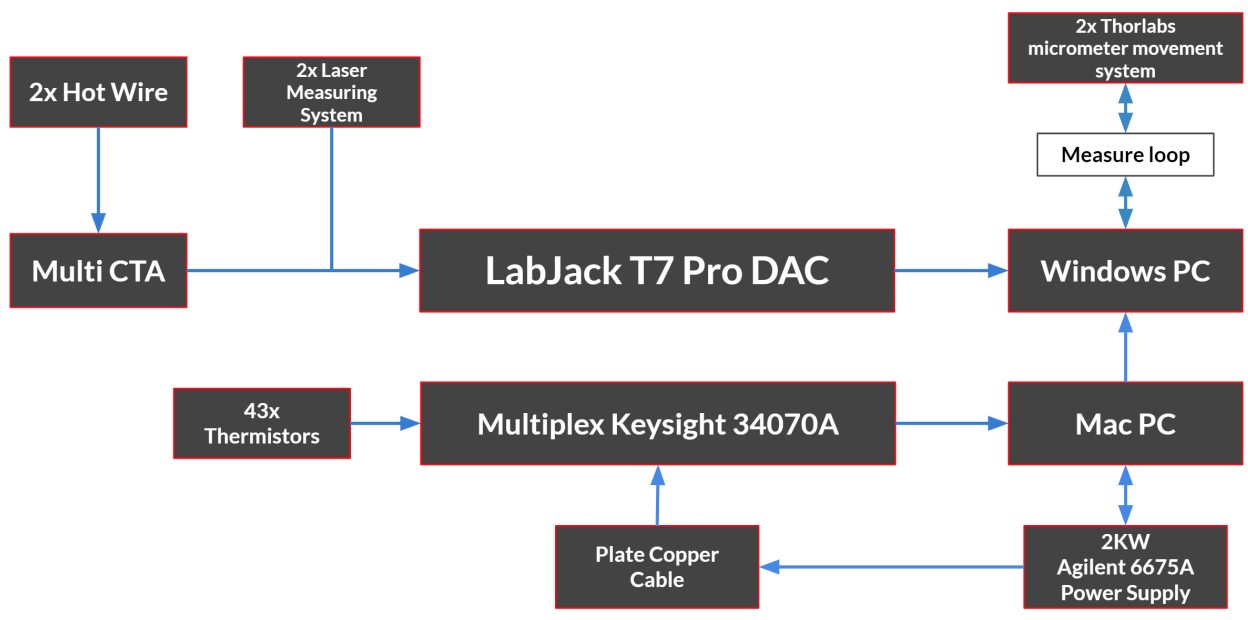

Figure 3.20: Schematic of connections layout for the temp controlled hot-wire velocity measurement

The hot-wires are connected to the Multi CTA that is responsible for the hot-wires feedback loop and is responsible for some signal processing. The CTA internal electronics does allow to apply a low-pass filter $(1,3,5,10 \mathrm{kHz}$ ), amplify the signal between (1 and $5 d B$ ) or shift the voltage between 0 and $+2 V$. The Multi CTA board has a base voltage that is applied and not subtracted by the system, this base voltage is also amplified with the gain control. In our range of velocity, we get a signal between $5 \mathrm{~V}$ and $8 \mathrm{~V}$. Schematic in fig. 3.20 .

Once the measurement position along the plate is set and the equilibrium temperature is reached we lower the hot-wire to the $z_{0}$ position. The data are then collected with a sampling rate of $10 \mathrm{kHz}$ for 120 seconds for each of the hot-wires. The hot-wires are then moved up to a new position and the measurement is repeated. We conduct measurements at 41 different positions that span from $0.2 \mathrm{~mm}$ to $400 \mathrm{~mm}$ above the plate surface. The minimum time necessary to measure the entire boundary layer velocity profile is 1 hour and 30 minutes, it can be more due to the region where the position is changed manually.

\subsubsection{Vertical thermistors measurements}

The temperature profile measures usually last around 24 hours, the data is collected with a sampling frequency of approximately $0.2 \mathrm{~Hz}$. The vertical thermistor bar is positioned approximately at the same $\mathrm{x}$ position where the velocity was taken at the center of the plate along the y-axis. We do not perform velocity and temperature measurements at the 
same time so that the different probes do not affect each other.

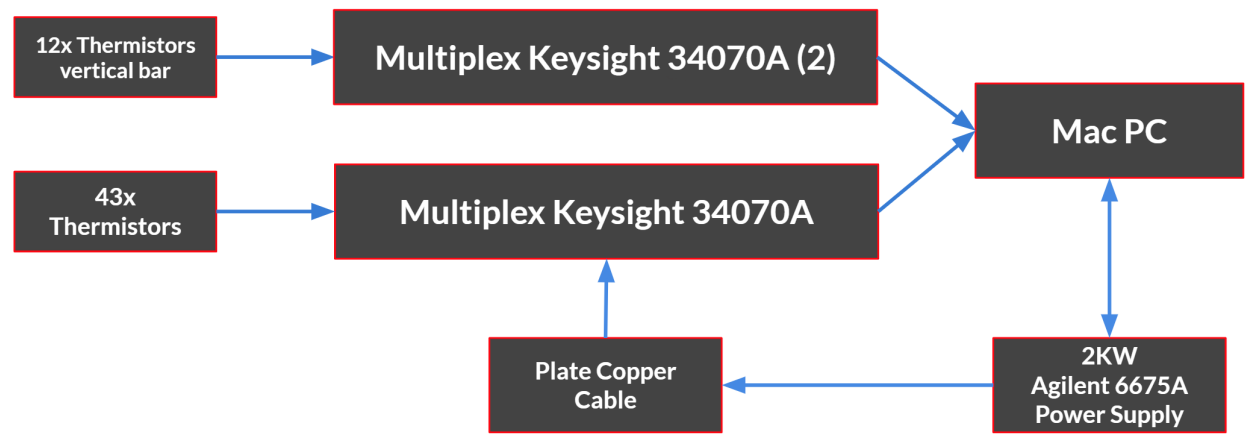

Figure 3.21: Schematic of connections layout for vertical temp profile measurement.

\subsubsection{Camera Image collection}

The temperature control and feedback loop are the same as described in section 3.7.1. In this setup, we used the windows computer to read the camera output using the proprietary software from Vision Research 3.22. The industrial smoke machine is configured to produce a pulse of smoke in time and the camera images are collected.

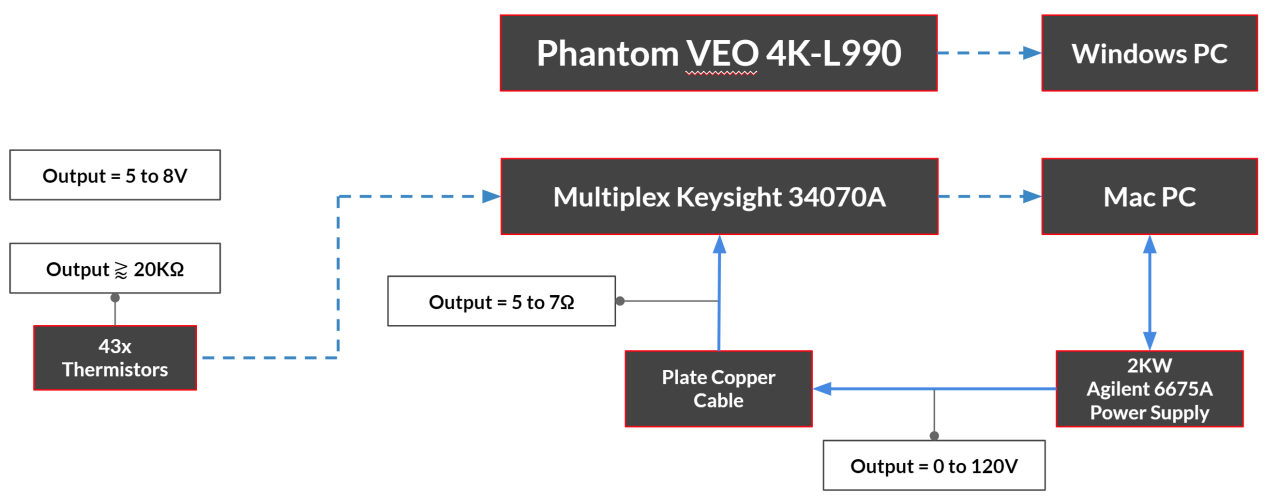

Figure 3.22: Schematic: connections layout for camera image collection

For every measurement, we record approximately 2 minutes of videos that are shoot at a frame rate of 100fps. We have tested export in RAW (12bit heavy file) and Apple ProRes (10bit medium size file). No relevant differences have been found in the post-processing, therefore the video has been exported in 10-bit ProRes. The videos are processed using professional video editing software (named DaVinci Resolve from Black-Magic). In the processing we do normalize the luminosity level, correct for the light falloff at various $\mathrm{x}$, 
increase the contrast, and apply small changes to enhance the flow structures. The same processing is applied to all the images. 


\section{Chapter 4}

\section{Data analysis and results}

Now, after we have provided the necessary background and explained in detail the experimental setup, we present in this chapter results from measurements of the velocity, the temperature field, and the heat flux from the surface of the plate. We will start in sec. 4.1 with velocity measurements in the boundary layer on top of an isothermal plate, i.e., without heating, followed by temperature and velocity measurements above a heated plate (sec. 4.2).

\subsection{Velocity boundary layer without heating}

In this section, we will present measurements of the streamwise velocity $u$ as a function of the vertical position $z$. From these measurements, we will calculate statistical quantities, such as averages, standard deviations, and higher-order moments, as well as power spectral densities and we investigate how these quantities change for different Reynolds numbers.

\subsubsection{Turbulent velocity boundary layer}

Using hot-wire we have measured the vertical profile of the streamwise velocity component above the plate at different bulk velocities $U_{0}$, i.e., different Reynolds numbers $\operatorname{Re}_{x}$. The velocity measurements are all done at distance $x=2.35 \mathrm{~m}$ from the leading edge of the adiabatic plate where the boundary layer starts to develop.

Using the shear stress velocity $u_{\tau}$ and the kinematic viscosity $\nu$ it is convenient to convert velocity and vertical position in a turbulent boundary layer in dimensionless wall 


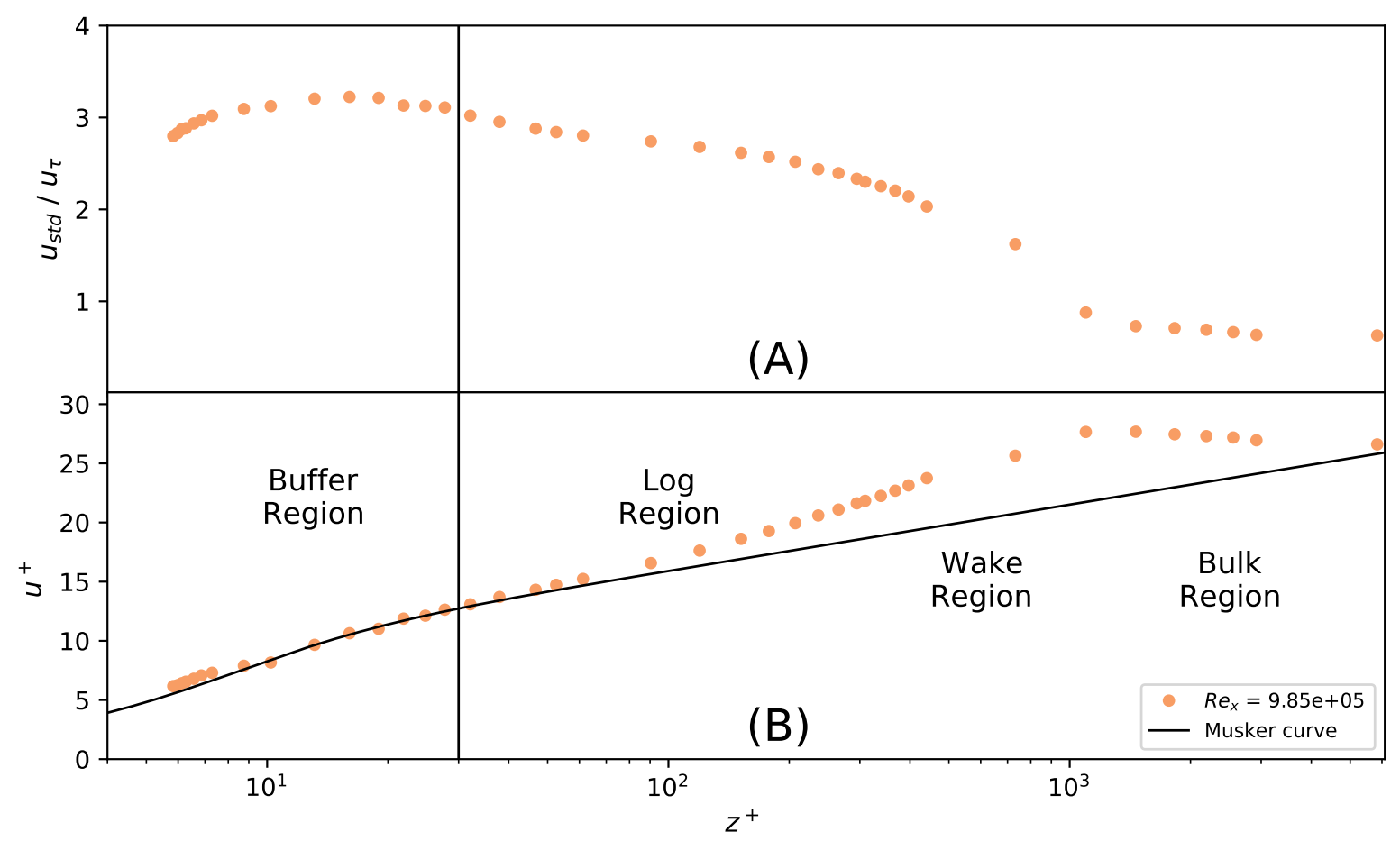

Figure 4.1: Turbulent velocity boundary layer profile. Shown are the time averaged velocities $(B)$ and the fluctuations expressed by the normalized standard deviation (A) as a function of the vertical distance from the plates top surface. The black line marks a Musker curve (eq. 2.23).

units

$$
u^{+}=\frac{u}{u_{\tau}}, \quad z^{+}=\frac{z \cdot u_{\tau}}{\nu},
$$

so that for sufficiently small $z^{+}$, all data $u^{+}\left(z^{+}\right)$collapse for very different $\operatorname{Re}_{x}$ onto a single master curve. This master curve can be well described by the Musker equation (eq. 2.23) introduced in sec. 2.3.2. We determine the skin friction velocity $u_{\tau}$ as a fit parameter by fitting our data to the Musker equation (eq. 2.23), whereas only data were used that belong to the buffer and the log-region as in fig. 4.1. During this fit, we also determined the precise distance between the hot-wire probe and the surface by adding or subtracting a constant $z_{0}$ to the assumed height $z$. However, we only allowed $z_{0}$ to be smaller than $0.5 \mathrm{~mm}$ since this was our estimated uncertainty for the measured distance between the hot-wire probe and the laser distance sensor.

Figure 4.1 shows the measurements of the streamwise velocity for the largest applied bulk velocity $U_{0}=6.29 \mathrm{~m} / \mathrm{s}\left(\operatorname{Re}_{x}=9.85 \times 10^{5}\right)$. Shown are the average velocities $u^{+}$ 
(fig. 4.1B), and the normalised standard deviations $u_{s t d} / u_{\tau}$ (fig. 4.1A), with

$$
u_{s t d}=\sqrt{\left\langle(u-\langle u\rangle)^{2}\right\rangle} .
$$

The velocity field follows the typical turbulent boundary layer velocity profile. The viscous sub-layer is expected to occur for $z^{+} \lesssim 5$ or so but it is not resolved here, as $z^{+}=5$ corresponds in this measurements to a physical distance of $z \approx 350 \mu \mathrm{m}$. While measurements at such small $z$ were in general possible, the averaged velocity, however, was below the velocity range at which the CTA gives reliable results. In the viscose sublayer the velocity gradient $\partial\langle u\rangle / \partial z^{+}$is steep (linear) and fluctuations $u_{\text {std }}$ are small and decrease towards the boundary (for $z^{+} \rightarrow 0$ ). Momentum is transported predominantly by diffusion with the viscosity as the diffusion constant.

Above the viscose sub-layer lies the buffer layer that occurs for distances from the plate $5 \lesssim z^{+} \lesssim 30$. Here, viscose diffusion is no longer the only mechanism for the momentum transport in a vertical direction away from the plate, but also advection becomes increasingly important. It is also in this region, where the velocity fluctuations in streamwise direction $\left(u_{s t d}\right)$ reaches a maximum. The buffer layer is introduced in literature as the zone in which the viscous sub-layer and log region merge and where the viscous and inertial forces are comparable [20].

The buffer layer is followed by the log-region for $30 \lesssim z^{+}$. There, the vertical momentum transport is mainly due to advection and viscosity does not play a role. The fluctuations $u_{\text {std }}$ decrease slightly inside the log-region but this decrease becomes weaker for even larger $z^{+}$and the curve flattens out. This is where the wake region [65] starts to occur, the most outer part of the boundary layer, before the flow field converges to the bulk flow. The wake region is a transitional region in which the slow and unstable log region velocity field mixes with the fast but stable bulk flow. The wake region position in wall units is a function of Reynolds number and is characterised by a steeper increase of the velocity with $z^{+}$in this semi-log presentation than in the log-region, but also by a sharp decrease of the fluctuations $u_{s t d}$. In our experiment we use an inflow velocity $U_{0}$ that is not big enough to allow for a strong separation between the viscose sub-layer and the wake region, resulting in a rather narrow log region.

The average velocity and the fluctuation as presented in fig. 4.1 are the most com- 
mon and easiest to calculate single-point statistical quantities that can be measured in the experiments. However, since our CTA probes take measurements at $10 \mathrm{kHz}$ we can calculate other statistical properties. For example, one can investigate the probability for the occurrence of different velocities, i.e., the velocity probability density function $P\left(u^{+}\right)$.

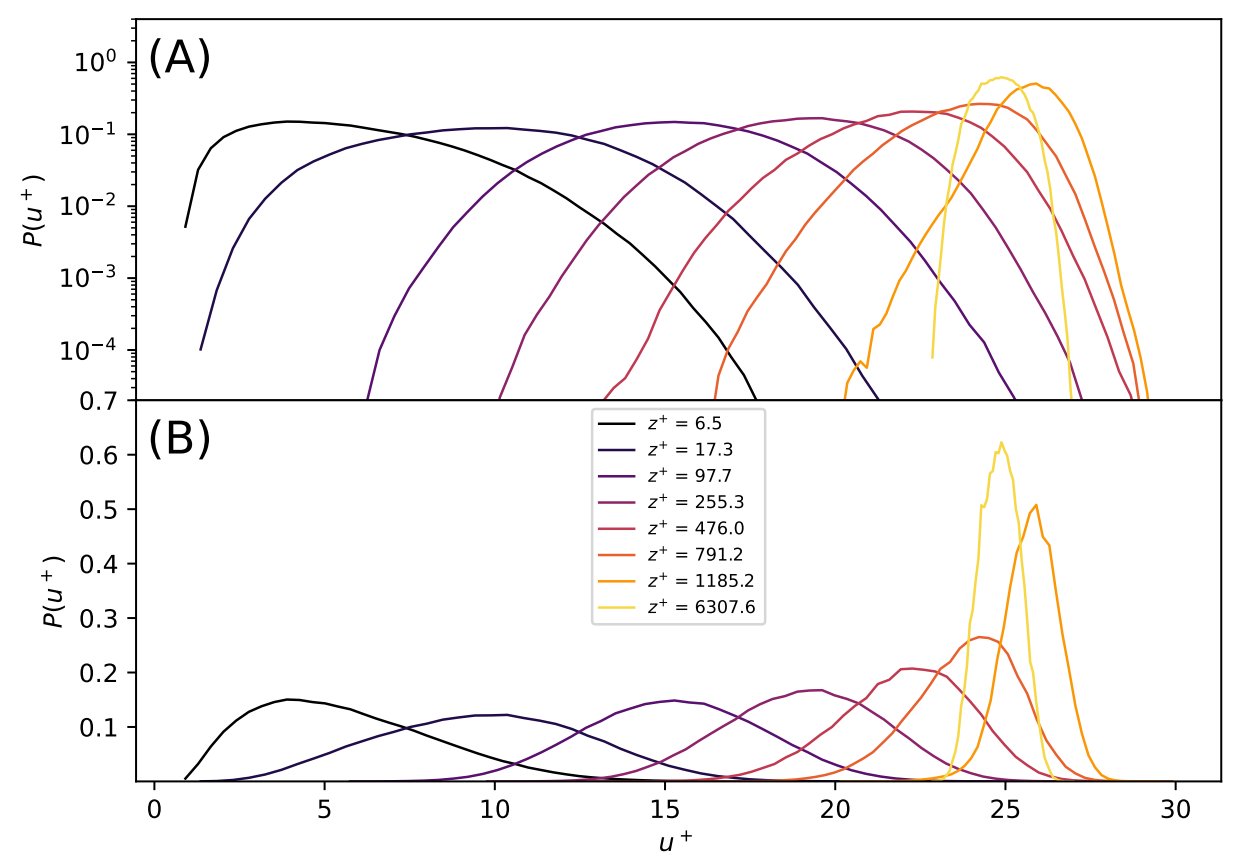

Figure 4.2: Probability density function of the streamwise velocity inside a turbulent boundary layer for $U_{0}=6.29 \mathrm{~m} / \mathrm{s}\left(R e_{x}=9.85 \times 10^{5}\right)$ and different heights (see legend). Top shows the data on a logarithmically scale $y$-axis, the bottom shows the same data on a linear scaled y-axis.

In fig. 4.2 we present the $\mathrm{P}(\mathrm{k})$ of the dimensionless velocity $u^{+}$for different vertical positions $z^{+}$(denoted by the line color, see legend).

One clearly sees that the $P\left(u^{+}\right)$changes qualitatively as a function of $z^{+}$and is characteristic for each of the regions of a fully developed turbulent boundary layer. Most notably - not at all surprising - the maxima of the $P\left(u^{+}\right)$moves towards larger $u^{+}$with increasing $z^{+}$. However, also the shape of the $P\left(u^{+}\right)$clearly changes with increasing $z^{+}$. When $z^{+}$is small (in the viscose sub-layer and the buffer layer) the $P\left(u^{+}\right)$is heavily skewed towards larger velocities and rather broad denoting a large $u_{\text {std }}$ as we have seen already above in fig. 4.1. The large skewness is somehow expected because velocities are restricted on the left by zero since negative velocities are not expected to occur. At least there is no known mechanism that forces a flow in opposite direction to the mean flow.

While still broad, the $P\left(u^{+}\right)$becomes symmetric and appears rather Gaussian inside 
the log-layer. For even larger $z^{+}$(inside the wake region), its width decreases but the $P\left(u^{+}\right)$now becomes skewed towards smaller velocities. This is intuitively explained by an upper bound for the velocity that is roughly $U_{0}$. The yellow $P\left(u^{+}\right)$in fig. 4.2 was taken at $z^{+}=6300$, which is outside the boundary layer and reflects the velocity in the bulk. Here, we see that the $P\left(u^{+}\right)$is rather narrow, indicating that the turbulence intensity is rather small in the bulk, but also symmetric, which is as well expected. What is unexpected, however, is that the mean velocity (i.e, the maximum) is significantly smaller than the maximum in the wake region. While we have seen this already above in fig. 4.1, in fig. 4.2 it becomes apparent that the difference between the two maxima is at least a standard deviation of the bulk $P\left(u^{+}\right)$. It is at this point unclear where the additional momentum comes from that accelerates the fluid beyond $U_{0}$.

The description in words above can be quantified by calculating higher moments, i.e., the skewness $\beta_{3}$, and kurtosis $\beta_{4}$ and see how they change along with the vertical position. Together with the $P\left(u^{+}\right)$, these quantities help to better characterize the turbulent boundary layer.

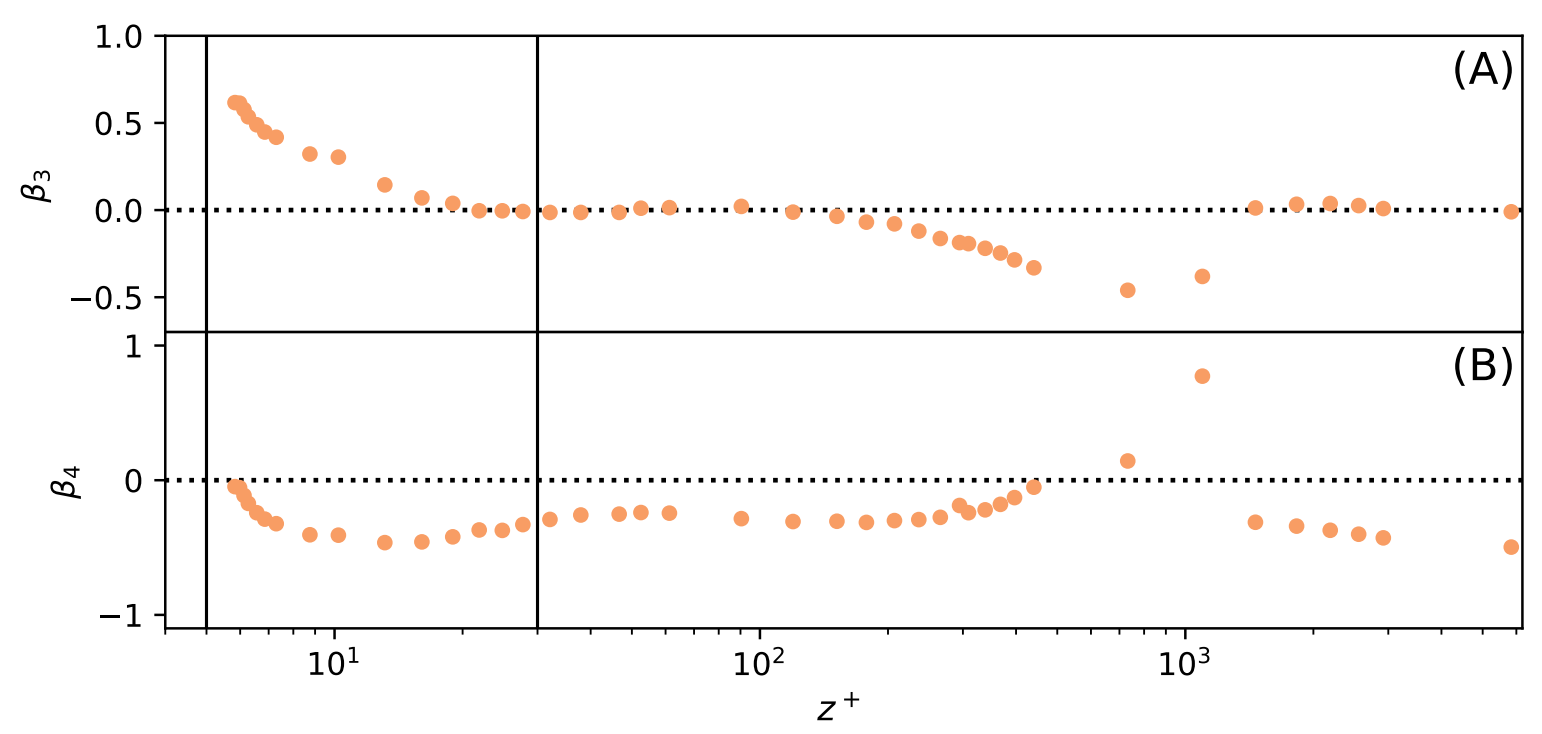

Figure 4.3: Skewness $\beta_{3}(A)$ and kurtosis $\beta_{4}(B)$ as function of vertical distance from the plate at $U_{0}=6.29 \mathrm{~m} / \mathrm{s}\left(R e_{x}=9.85 \times 10^{5}\right)$.

Figure 4.3 shows the skewness (top), and kurtosis (bottom) of the velocity fluctuations $u^{\prime}=u-\langle u\rangle$. The skewness $\beta_{3}[66]$ refers to the asymmetry of the $P\left(u^{+}\right)$and is defined 
as:

$$
\beta_{3}=\frac{\left\langle\left(u^{\prime}\right)^{3}\right\rangle}{u_{s t d}^{3}}
$$

A perfectly symmetric distribution has $\beta_{3}=0$ and its median and the average value coincide. When the median value is higher than the mean, the majority of the measurements are above the mean, and $\beta_{3}>0$. When the median value is lower than the mean, the majority of the measures are below the mean, and $\beta_{3}<0$. The kurtosis $\beta_{4}$ [66] provides information about the tails of the probability, i.e., the probability of very extreme events. It is defined as:

$$
\beta_{4}=\frac{\left\langle\left(u^{\prime}\right)^{4}\right\rangle}{u_{s t d}^{2}}-3
$$

so that for a normal distribution it is $\beta_{4}=0$.

We observe $\beta_{3}>0$ in the viscous sub-layer and part of the buffer region. This is due to the lower range of accessible velocities induced by the non slip condition at the wall, resulting in an asymmetric distribution. In the log region this limitation is not present and therefore the skewness approaches zero, in accordance with the symmetric $P\left(u^{+}\right)$shown in fig. 4.2. In the wake region, we observe $\beta_{3}<0$, because the bulk flow is (almost) laminar and therefore the accessible velocities are limited from above. In the bulk, $\beta_{3} \approx 0$, since there, the flow is only very weakly turbulent of low intensity and the velocity fluctuations are symmetric.

A negative kurtosis is observed in almost the entire boundary layer. Solely in the wake region, the kurtosis becomes positive, where slow fluid from the boundary layer mixes with fast fluid from the bulk. Since in this region, the fast and slow fluids are not well mixed, large patches of fast and slow fluid exists, causing sudden changes in the detected velocity and hence a high probability of detecting velocities that are very different from the mean velocity at this $z^{+}$. The negative kurtosis does represent a flow with less extreme events where the fluctuations are more constrained, with a positive kurtosis we do expect an increase of extreme events that reflect a longer-tail in the distribution. In turbulent isotropic flows, it is expected to find slightly negative values of kurtosis [67]. Our findings for fig. 4.3 agree with large eddy simulations by Stevens et al., [68] and experimental measurements by Durst 1987 [69] for a turbulent boundary layer. 


\section{Power spectral density}

As we discussed in chapter 2.2, for isotropic and homogeneous turbulent flows, Kolmogorov predicted a $E(k) \propto k^{-5 / 3}$ scaling for the spectral distribution of turbulent kinetic energy. We do expect that when the $k^{-5 / 3}$ scaling range can be clearly seen, the turbulent flow can be considered homogeneous and isotropic.

To calculate the kinetic energy spectrum, we use the Taylor frozen turbulence hypothesis [6]. It assumes the turbulent eddies to be frozen in and transported by the flow as they passed the sensor. Therefore, from measurements of a certain quantity at a fixed position as a function of time, one can calculate the streamwise spatial variation of this quantity at a fixed moment in time.

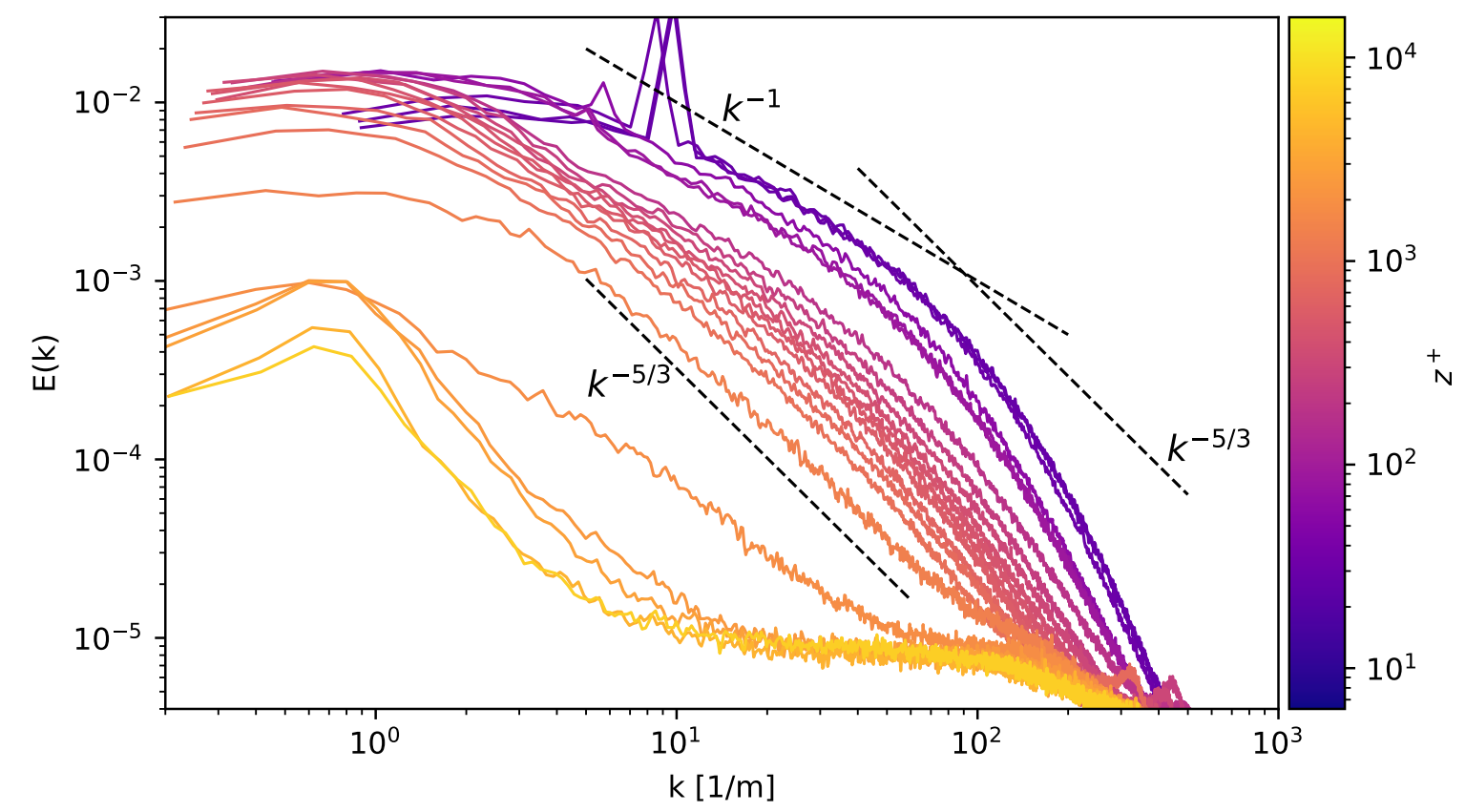

Figure 4.4: Energy spectrum at different vertical positions $\left(z^{+}\right.$according to color bar) inside the boundary layer and for $R e_{x}=9.85 \times 10^{5}$.

Figure 4.4 shows the power spectral density at different $z^{+}$positions inside the boundary layer, with the last two (orange) in the bulk. With the probe at our disposal, we measured the velocity component along the flow direction, the $x$ axis. All the presented power spectral density in this work, are therefore representing the one-dimensional power spectral distribution along with the flow.

In the presented data, the measurements closest to the wall were taken inside the buffer region. There, the flow is not isotropic and we do not see a clear $k^{-5 / 3}$ scaling 
for any sufficiently large $k$-range. Instead, we clearly see a $k^{-1}$ scaling for wavenumbers somewhere in the range $5<k<50 \mathrm{~m}^{-1}$ or so. This observation will be discussed later in this chapter. A sharp decrease of the spectral energy occurs at around $200 \mathrm{~m}^{-1}$, where the energy dissipation sets in.

Looking now at the boundary layer log region (purple) we do see the development of the $k^{-5 / 3}$ scaling that does coexist with the $k^{-1}$ already spotted in lower positions inside the boundary layer. The existence of the $k^{-5 / 3}$ scaling does suggest that the flow becomes more homogeneous and isotropic with the growth of this region, which is in accordance with the vanished skewness $\beta_{3}$ and the slightly negative kurtosis $\beta_{4}$ shown above.

Approaching the wake and bulk region we see a drop of the turbulent kinetic energy. This is of course expected. The bulk is nearly laminar and therefore the turbulent kinetic energy contained in it is rather small (note the logarithmically scaled y-axis in fig. 4.4).

In our study, we can see a clear $-5 / 3$ scaling from $z^{+}>250$, this scaling disappears once we exit the boundary layer. According to what we discussed in chapter 2.2 about the Kolmogorov energy scaling, the inertial subrange is expected to exist just under specific conditions. We can then say that these conditions, i.e., isotropy and homogeneity, exists on sufficiently small length scales (i.e., large wavenumbers) and sufficiently large distances from the wall $\left(z^{+}>250\right)$. Due to the energy cascade from large to small structures, isotropy is recovered on small scales. Note that in our experiment the bulk flow is nearly laminar and therefore the inertial subrange is not expected to be found at the end of the wake region.

In the previous paragraph, we have observed, where $E(k) \propto k^{-1}$ for certain $k$-ranges. Tchen in 1953 [70], [71], has been the first to predict the potential existence of a $k^{-1}$ scaling via the spectral budget equation. The same $k^{-1}$ scaling has been obtained later with other approaches like asymptotic matching between the velocity spectrum inside the boundary layer region velocity spectra, and dimensional analysis in [31], [72], [73].

This $k^{-1}$ power-law has not been observed uniformly in all the experimental works during the years driving to an open discussion about its existence. This regime is supposed to be found in the power spectral density of the streamwise velocity component $u_{x}$. The increasing anisotropy and inhomogeneity close to the bounding surface result in a different decay of the vertical and horizontal component of the velocity fluctuation. When the difference between those decay is not negligible, the flow develops a different energy 


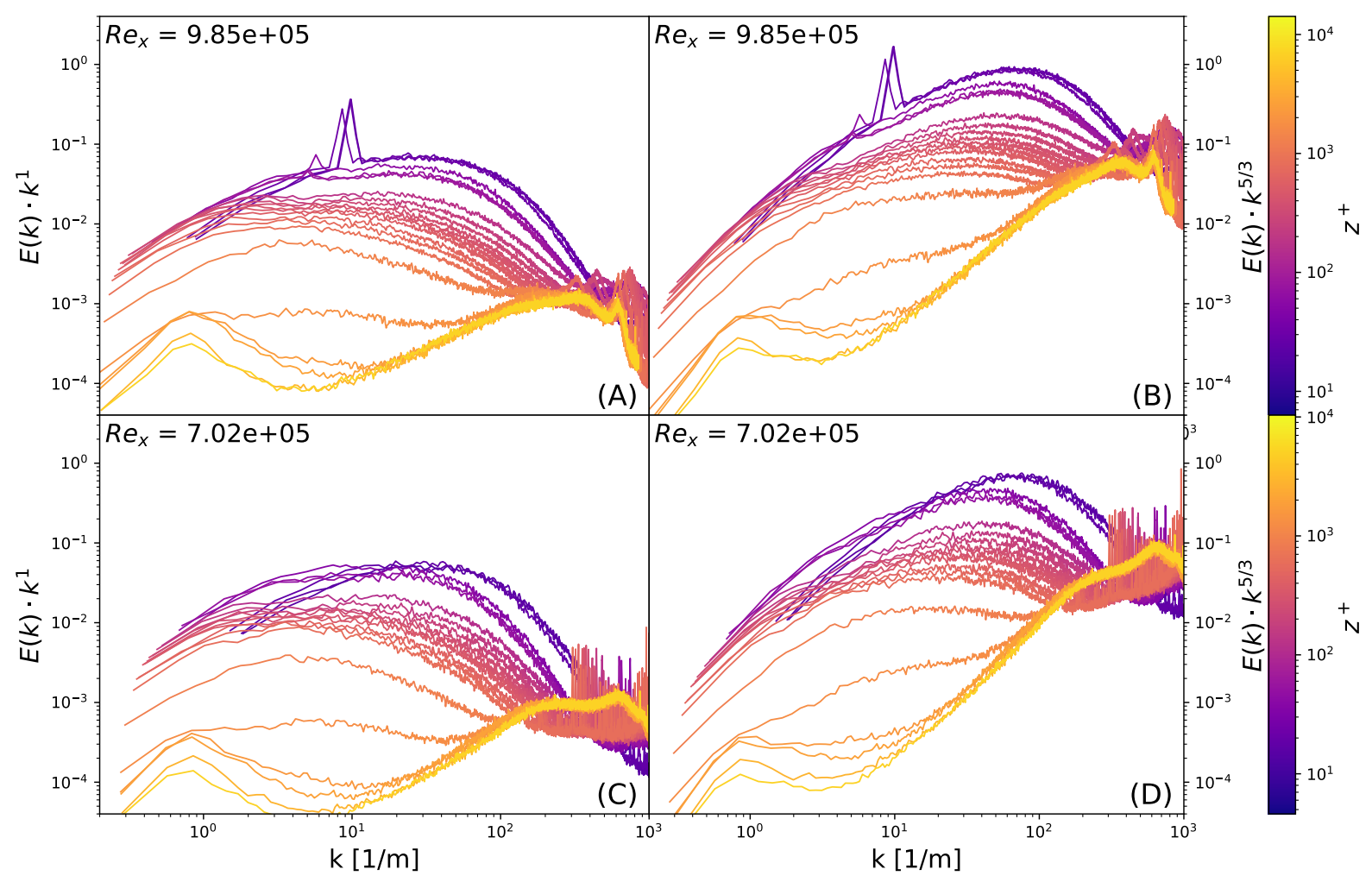

Figure 4.5: Pre-multiplied power spectral density. (A) and (C) represent the power spectral density for $R e_{x}=9.85 \times 10^{5}$, (B) and $(D)$ represent the power spectral density for $R e_{x}=7 \times 10^{5}$.

cascade scaling proportional to $k^{-1}$ somewhere between the energy injection range and the inertial subrange. Nickels in 2005 [74] presented an experimental investigation of the $k^{-1}$ power-law, and suggests that it can only be observed for $z^{+}>100$ and $\operatorname{Re}>1 \times 10^{6}$.

We present a pre-multiplied power spectral density for two different flow velocities: $U_{0}=6.29 \mathrm{~m} / \mathrm{s}$ with $R e_{x}=9.9 \times 10^{5}$ in fig. 4.5 (A and B), $U_{0}=4.48 \mathrm{~m} / \mathrm{s}$ with $R e_{x}=7 \times 10^{5}$ in fig. 4.5 (B and D). These power spectra $E(k)$ are pre-multiplied by $k$ (A and $\mathrm{C}$ ) and $k^{5 / 3}$ (B and D), the respective $k^{-1}$ and $k^{-5 / 3}$ scaling will result in a flat region where they occur.

For $R e_{x}=9.9 \times 10^{5}$ we can recognize the $k^{-1}$ - scaling for heights from $z^{+}=25.3$ extending up to $z^{+}=476$. The $k^{-5 / 3}$ - scaling is observed for heights in the range from $z^{+}=223.8$ to $z^{+}=791.2$. When $R e_{x}=7 \times 10^{5}$ we can recognize the $k^{-1}$ - scaling for heights from $z^{+}=19.5$ and extending up to $z^{+}=243$. The $k^{-5 / 3}$ - scaling is observed for heights in the range from $z^{+}=71$ and extend up to $z^{+}=577$. Clearly, at various heights $z^{+}$both scaling are observed. There, at small wavenumbers, where the flow is still anisotropic $k^{-1}$ is found, while for sufficiently large wavenumbers the flow has regained 
isotropy resulting in $k^{-5 / 3}$.

This allows us to confirm the existence of the $k^{-1}$ region in our measurement. It has been observed between the energy injection range and the inertial subrange, as observed by Perry in 1986 [31], Nickels in 2005 [74] and others. In our study, we observed a $k^{-1}$ region measured at a minimum vertical position $z^{+}=71$ and a minimum value of $R e_{x}$ that do not match with the minimum expected according to the Nickels 2005 [74] study that suggested higher minimum values for both of those quantities to allow the $k^{-1}$ region to be visible. This result to the best of our knowledge does represent an unprecedented experimental finding of the $k^{-1}$ scaling for such a low value of $R e_{x}$ and $z^{+}$.

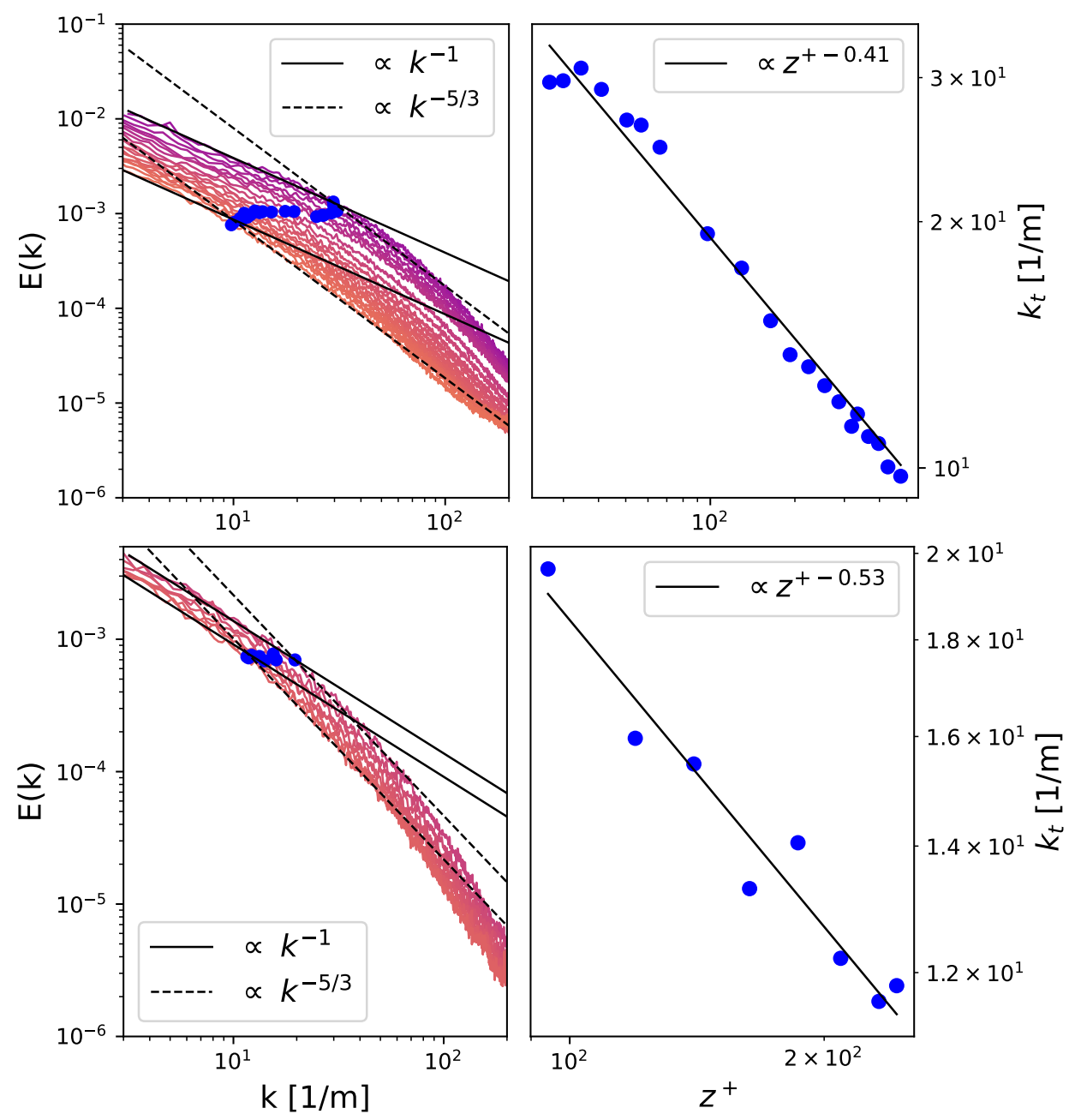

Figure 4.6: Dots represent the transitional wave number where the $k^{-1}$ ends and the $k^{-5 / 3}$ starts. Top plots represents data collected with $R e_{x}=9.9 \times 10^{5}$, bottom plots represents data collected with $\operatorname{Re}_{x}=7 \times 10^{5}$.

To further study the transition between these two energy scaling regimes, we fit a 
function of the form

$$
E(k)=\left\{\begin{array}{cc}
A k^{-1} & \text { for } k<k_{t} \\
B k^{-5 / 3} & \text { for } k \geq k_{t}
\end{array}\right.
$$

to the data with fit parameters $A, B$, and the transition point $k_{t}$. In fig.4.6 we present our method applied to both $R e_{x}=9.9 \times 10^{5}$ (Top) and $R e_{x}=7 \times 10^{5}$ (Bottom). The measurements in which we can clearly see that $k^{-1}$ range in $R e_{x}=7 \times 10^{5}$ is too narrow and therefore we can derive only a small amount of points. Nevertheless we wanted to present the analysis for completeness. We have found that the transitional wave number between the two regimes $k^{-1}$ and $k^{-5 / 3}$ depends on the distance according to a a powerlaw $z^{+} \propto z^{\alpha}$, with $\alpha \approx 0.41$ for $\operatorname{Re}_{x}=9.9 \times 10^{5}$ and $\alpha=0.52$ for $\operatorname{Re}_{x}=7 \times 10^{5}$. These exponents are somehow surprising as from arguments in sec. 2.3.2, we would have expected an exponent closer to unity.

\subsubsection{Velocity boundary layer in transitional regime}

After we have presented a detailed analysis of the fully turbulent boundary layer for the largest $R e_{x}$, we want to investigate now the boundary layer at smaller $\operatorname{Re}_{x}$. Hence, in this section, we present our observations for lower bulk flow velocities $U_{0}$, while the measurements were still taken at position $x=2.35 \mathrm{~m}$ away from the leading edge. We will see that for the smallest $U_{0}$ the boundary layer cannot be considered fully turbulent anymore, but is also not laminar. We will then study the main characteristics of the boundary layer in a quasi-turbulent regime and highlight the differences that can be found in a comparison with the fully turbulent ones. The transition of the boundary layer from laminar to turbulent is not sharp and well defined, therefore we may refer to the non-turbulent, but fluctuating, boundary layer as "quasi-turbulent" or "transitional".

Our experimental setup allows us to perform measurements at very small distances from the surface, which result in theoretically extremely low $z^{+}$. In reality, despite we can collect these data extremely close to the wall surface, we are forced to discard some of them due to the extremely low flow velocity at these distances that are out of the operative range of the CTA probe. We discussed the hot-wire technical limitations in sec. 3.3.1. The analysis of the influence of the surface heat on the $\mathrm{k}-1$ regime is presented in sec. 4.2.1. 


\section{Average velocity profiles and fluctuation}

In fig. 4.7 we present the average velocity (B) and the velocity fluctuations (A) as a function of $z^{+}$for five different $\operatorname{Re}_{z}$ in the range $4.7 \times 10^{4} \leq \operatorname{Re}_{x} \leq 9.9 \times 10^{5}$.

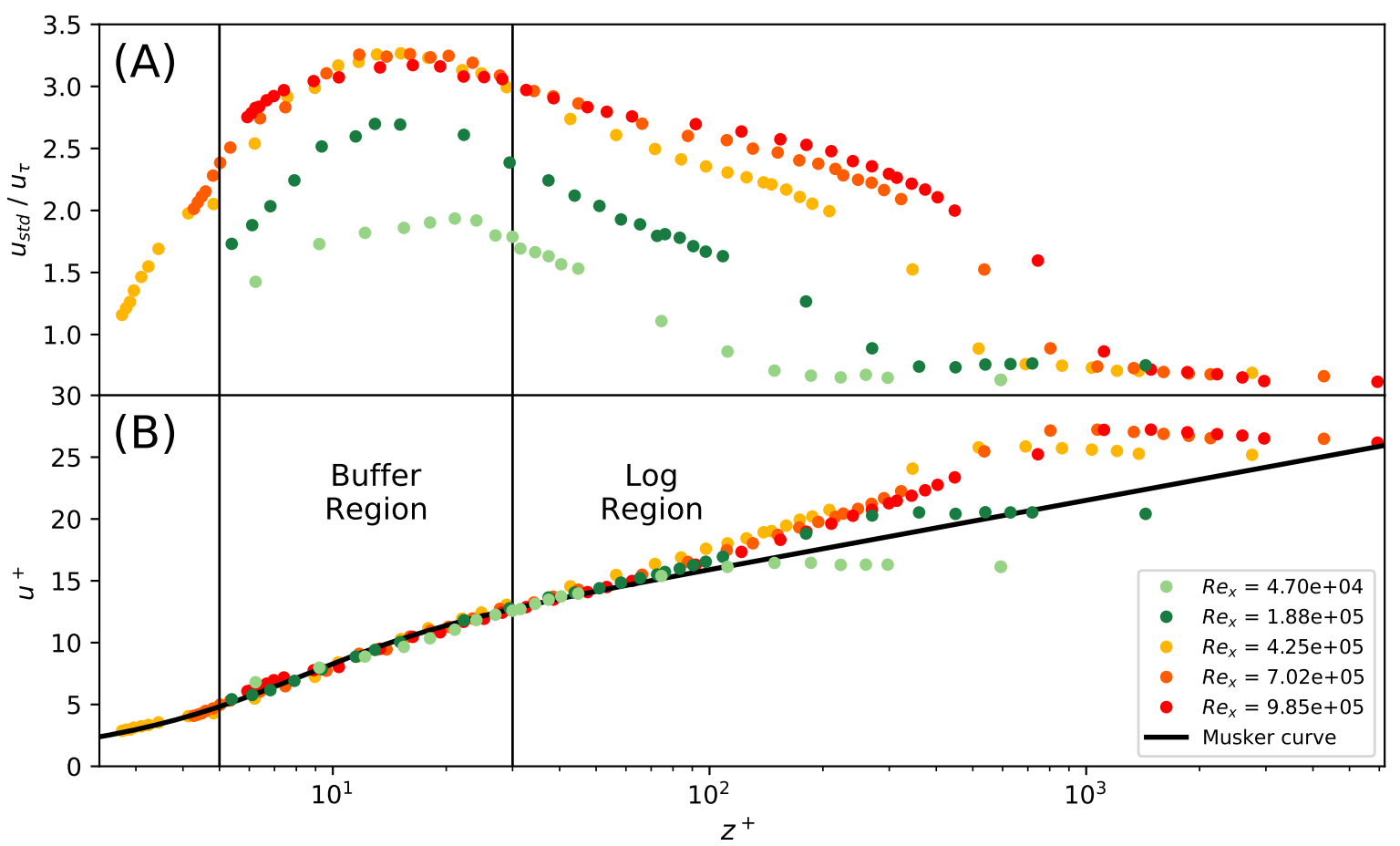

Figure 4.7: Comparison of turbulent boundary layer and transitional boundary layer. Shown are the velocity fluctuations (A) and the average velocity (B) as function of the distance to the wall $z^{+}$.

Note, while $u_{\tau}$ was calculated from a fit to the Musker equation as described above for large $\mathrm{Re}_{x}$, for the smallest $\mathrm{Re}_{x}$, the inner layer was large enough so that we could measure the initial velocity close to the plate and hence we could determine the shear velocity directly as $u_{\tau}=\partial u /\left.\partial z\right|_{z=0}$. The skin friction velocities for the five $\operatorname{Re}_{x}$ shown in fig. 4.7 are listed in table 4.1 .

\begin{tabular}{||c||c|c|c|c|c||}
\hline$R e_{x}$ & $4.70 \times 10^{4}$ & $1.88 \times 10^{5}$ & $4.25 \times 10^{5}$ & $7.02 \times 10^{5}$ & $9.85 \times 10^{5}$ \\
\hline$u_{\tau}[\mathrm{m} / \mathrm{s}]$ & 0.0225 & 0.054 & 0.104 & 0.161 & 0.221 \\
\hline
\end{tabular}

Table 4.1: Experimentally determined wall shear velocities $u_{\tau}$ for different $R e_{x}$.

For the three largest $\mathrm{Re}_{x}$ the boundary layer is rather turbulent, while for the smallest $\operatorname{Re}_{x}$ a clear $\log$ region or a wake region are not visible. In fig. 4.7 we clearly see that a reduction in $\operatorname{Re}_{x}$ affects first the wake region. This is of course expected because we 
plot flow quantities in wall units derived from the velocity gradient at the wall and hence the inner layer is self-similar. The boundary layer height and the wake region moves to smaller $z^{+}$with increasing $\operatorname{Re}_{x}$ lowering the $z^{+}$-range where we observe the log-law. Also, for smaller $\mathrm{Re}_{x}$, the average velocity diverges from the Musker profiles at smaller $z^{+}$, showing a steeper increase in the wake region, and reaches finally the lower bulk velocity $u^{+} \approx U_{0} / u_{\tau}$ at smaller $z^{+} \approx \delta u_{\tau} / \nu$.

We also see the absence of a clear wake region for the lowest bulk velocity at $\operatorname{Re}_{x}=$ $4.7 \times 10^{4}$. There is no overshoot visible, where the velocity increases steeper than the logarithmic increase. Instead, the data points diverge from the Musker curve and reach asymptotically the bulk velocity. The absence of this outer layer is a clear indication that the scale separation is not large enough to consider the boundary layer fully turbulent. Interestingly, the lower part of the velocity profile still agrees well with the Musker function at $z^{+}$where the buffer and the log-region occur for turbulent conditions. In this regard, we also point out again that the log-region can be considered a transition zone between the inner layer where viscosity dominates and the outer layer where von Karman's velocity deficit law holds.

Figure 4.7(A) shows the velocity fluctuations that show stronger differences between quasi turbulent and fully turbulent regimes. When $R e_{x}>1.88 \times 10^{5}$ we see a well defined peak in the buffer region for $5<z^{+}<30$ centered around $z^{+} \approx 15$ with a height that is rather similar for the three largest $\operatorname{Re}_{x}$. For $z^{+}>30$ the decrease of $R e_{x}$ results in a decrease of the boundary layer height measured in wall units as clearly seen by the drop in $u_{s t d}$ at $z^{+}=\delta u_{\tau} / \nu$. Consequently, the wake region becomes smaller and the fluctuations are reduced. In particular, the small bump disappears with decreasing $\mathrm{Re}_{x}$. While it is still visible at $\operatorname{Re}_{x}=4.25 \times 10^{5}$, it is absent at $\operatorname{Re}_{x}=1.88 \times 10^{5}$. In addition, at $R e_{x}=1.88 \times 10^{5}$ also the maxima at $z^{+} \approx 15$ is considerably smaller as for the larger $\operatorname{Re}_{x}$. For even lower shear stress at $R e_{x}=4.7 \times 10^{4}$ the localised main peak at $z^{+} \approx 15$ decreases in height but also expands in width, resulting in a "plateau-like" region between $z^{+}=10$ and $z^{+}=25$.

In figure 4.8 we show velocity profiles for the two smallest $\operatorname{Re}_{x}$ and compare these profiles with the Musker curve [28], representative for a fully turbulent boundary layer, and with different Blasius profiles, i.e., the solution of eq. 2.11 [17] that represent the expected velocity profile for a laminar boundary layer. For the Blasius curves, we assumed either 


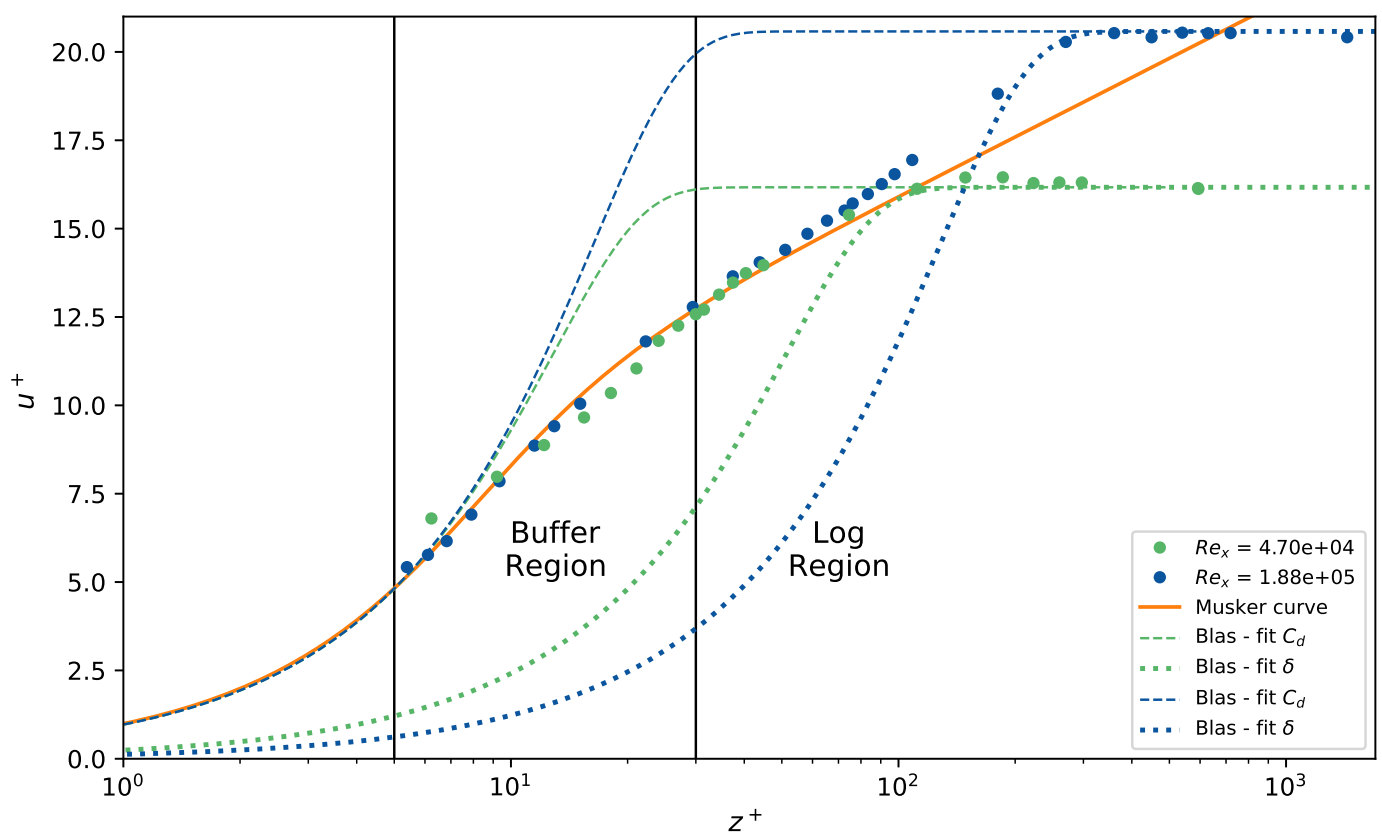

Figure 4.8: Average velocity profile for the two smallest Re $e_{x}$ (bullets) compared with the Blasius solutions with the same skin friction (dashed lines) as well as with the Blasius solutions that have the same boundary layer height $\delta$ (dotted line). The solid orange curve marks the Musker.

the same skin friction $\tau_{w}$ as for our measurements (dashed lines) or the same boundary layer height (dotted lines). In this plot, one sees that both our measured profiles are more similar to the turbulent profile than to the Blasius solution. Even though both curves are in the transitional regime, they show qualitative differences. For the lower $R e_{x}$ and $z^{+}<30$ we do see a different curvature in the scaling (the slope in the semi-log plot) of the velocity. This "quasi-linear" growth is uniquely seen in this specific data- set. The same cannot be said for $R e_{x}=1.88 \times 10^{5}$ that follows better the Musker curve. Also, in the wake region, the quasi turbulent regime $R e_{x}=1.88 \times 10^{5}$ still shows the characteristic overshoot as it is characteristic for a fully turbulent boundary layer, while this overshoot is absent for $R e_{x}=4.7 \times 10^{4}$.

\section{The distribution of velocity fluctuations}

In fig. 4.9 we present the probability density function for $\operatorname{Re}_{x}=4.7 \times 10^{4}$, which should be compared with the one for a fully turbulent boundary layer shown above in fig. 4.2. On the first look, we do not see one qualitative difference between these two figures, which is that the location of the maxima is a monotonic function of $z^{+}$here, while in fig. 4.2 the largest 


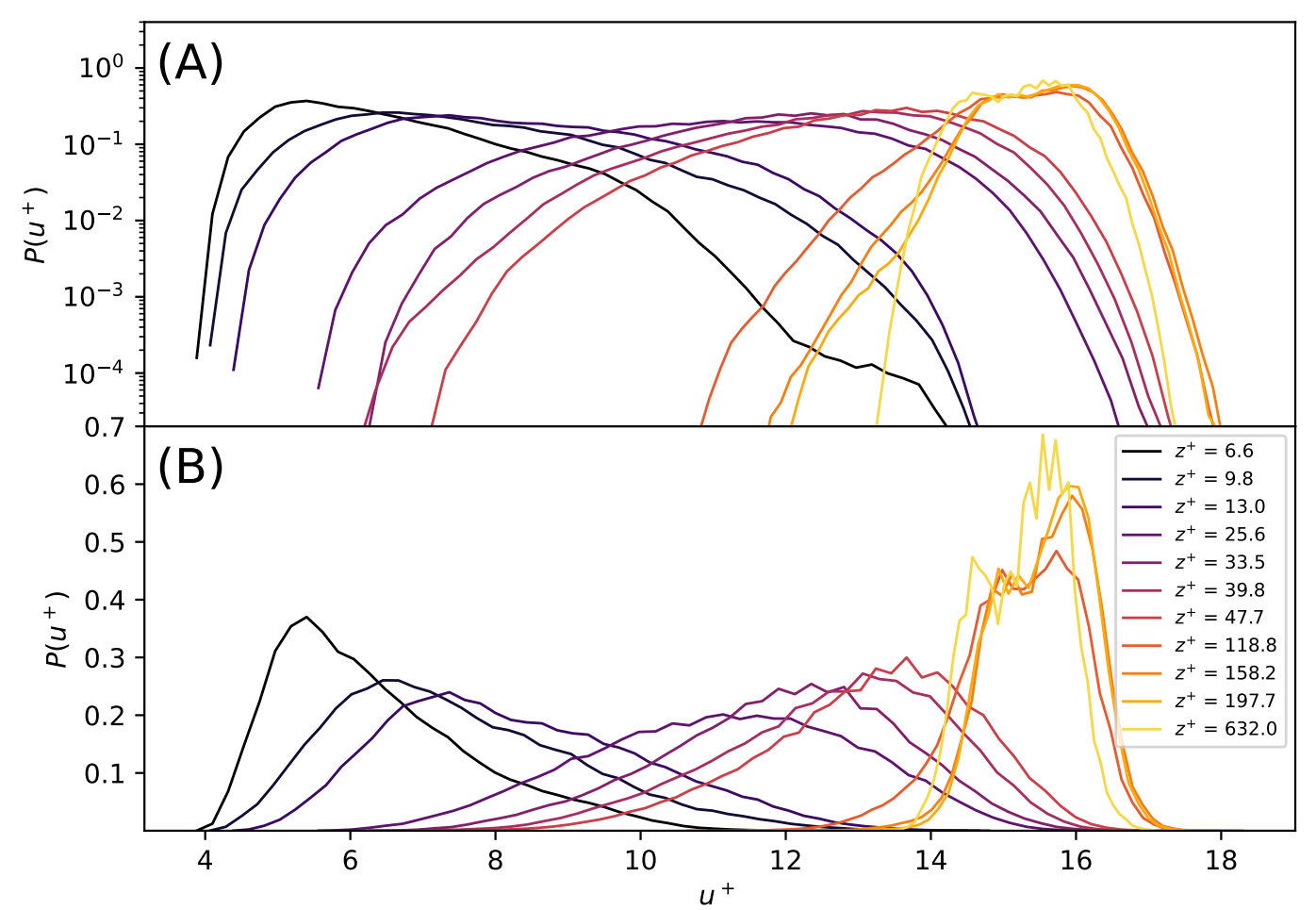

Figure 4.9: Probability density function of transitional boundary layer for $U_{0}=0.3 \mathrm{~m} / \mathrm{s}\left(R e_{x}=\right.$ $\left.4.7 \times 10^{4}\right)$.

velocities were measured inside the most external regions of the boundary layer, which were larger than in the bulk. Here, the bulk flow shows the largest velocities. Furthermore, the peak of the PDF in the bulk is less sharp and wider than for the larger flow speed. It can be speculated that the slow rotation rate of the propeller causes detectable velocity variations in time.

For a quantitative analysis, we compare in fig. 4.10 the skewness $\beta_{3}(\mathrm{~A})$ and kurtosis $\beta_{4}$ (B) at different positions for the fully turbulent and quasi turbulent boundary layer. Lets first have a look at the skewness $\beta_{3}$ in fig. 4.10 (A). The fully turbulent boundary layers $\left(R e_{x}>1.88 \times 10^{5}\right)$ shows a very consistent trend for $z^{+}<200$, with very similar values of $\beta_{3}$ at a given $z^{+}$. Differences between different $\operatorname{Re}_{x}$ are visible only for $z^{+}>200$, which is as we discussed above, because only the inner layer is self-similar in wall units, whereas the outer layer (the wake region) shifts towards smaller $z^{+}$with decreasing $\operatorname{Re}_{x}$. This is clearly visible in fig. 4.10 since the wake region starts where $\beta_{3}$ becomes negative. It is important to point out that this negative region is relatively small and well defined. Different considerations can be done for quasi-turbulent boundary layers with a $\beta_{3}$ for $R e_{x}=1.88 \times 10^{5}$ extremely close to the data for the fully turbulent boundary layer for 


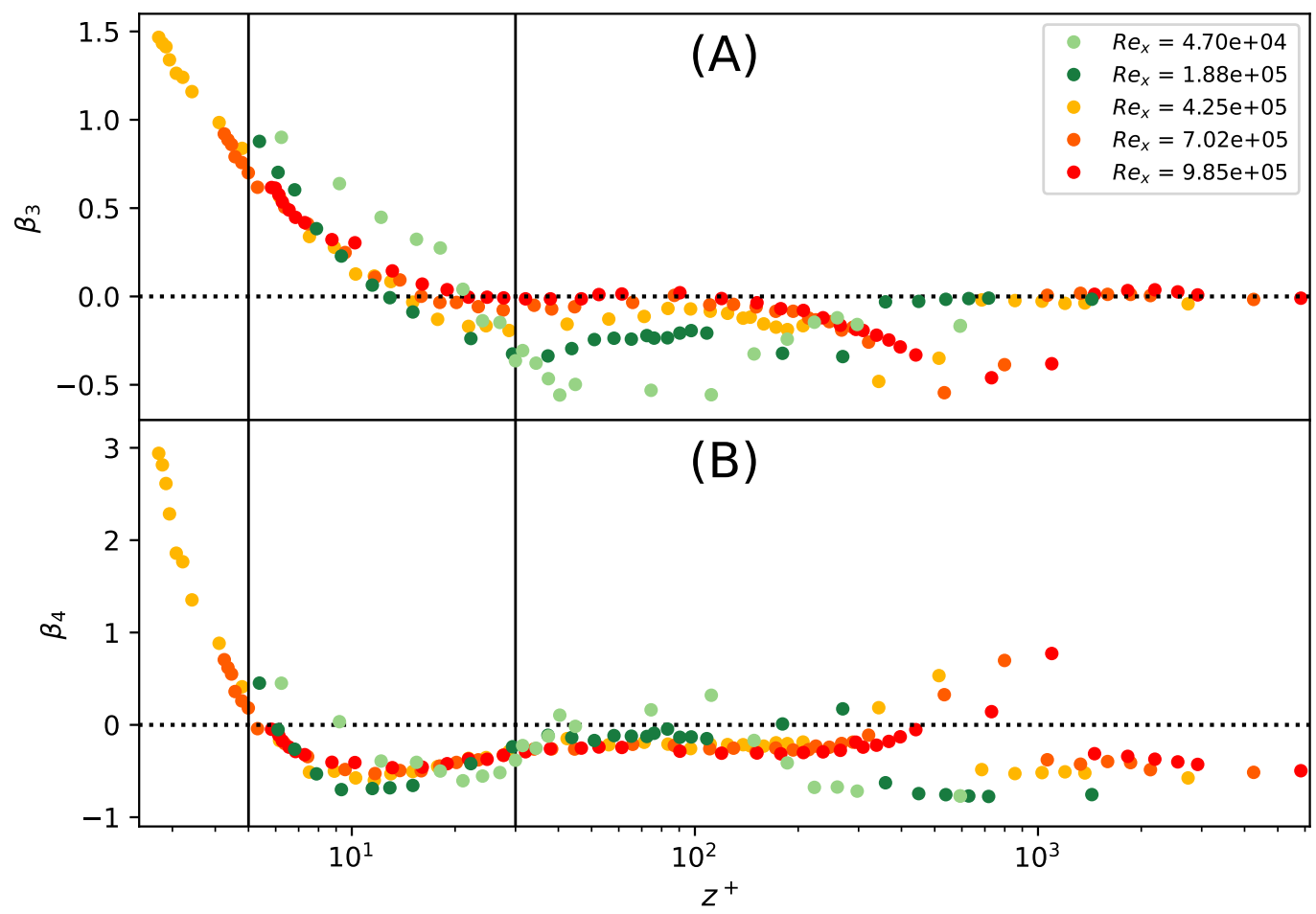

Figure 4.10: Skewness and kurtosis as function of distance from the plate for different $R e_{x}$.

$z^{+}<30$ and lower than the turbulent boundary layer ones for $30<z^{+}<300$. It can also be noticed that for this data-set it is extremely hard to define the characteristic wake region from the skewness resulting in what seems to be a more distributed transition beginning already at $z^{+} \approx 30$ and sustained along the rest of the boundary layer. When we look at $\beta_{3}$ for $R e_{x}=4.7 \times 10^{4}$, we can see a different scaling of $\beta_{3}$ for $z^{+}<30$ resulting in a quasi-logarithmic decline of the data with $z^{+}$. Furthermore, there is no region where the $\beta_{3}$ is constant close to 0 . We have shown that for $R e_{x}=4.7 \times 10^{4}$ the transition between the inner boundary layer region and the outer region begins already at $z^{+} \approx 30$, and this can also be seen in the skewness, which shows a less sharp wake region transition. Instead of a sharp minimum, one it exhibits a wide (on the log-scale) distributed minimum that approaches the value of 0 only in the bulk.

Moving to the kurtosis $\beta_{4}$ fig. 4.10 (B), the fully turbulent boundary layer $\left(R e_{x}>\right.$ $\left.1.88 \times 10^{5}\right)$ is presented to us again with values that overlap extremely well in the buffer region $z^{+}<30$, as well as in the $\log$ region for different $\mathrm{Re}_{x}$. The only difference in the kurtosis for this data-set is again represented by the different position of the wake region on the $z^{+}$-axis resulting in a peak of the kurtosis that is shifted to the left for decreasing 


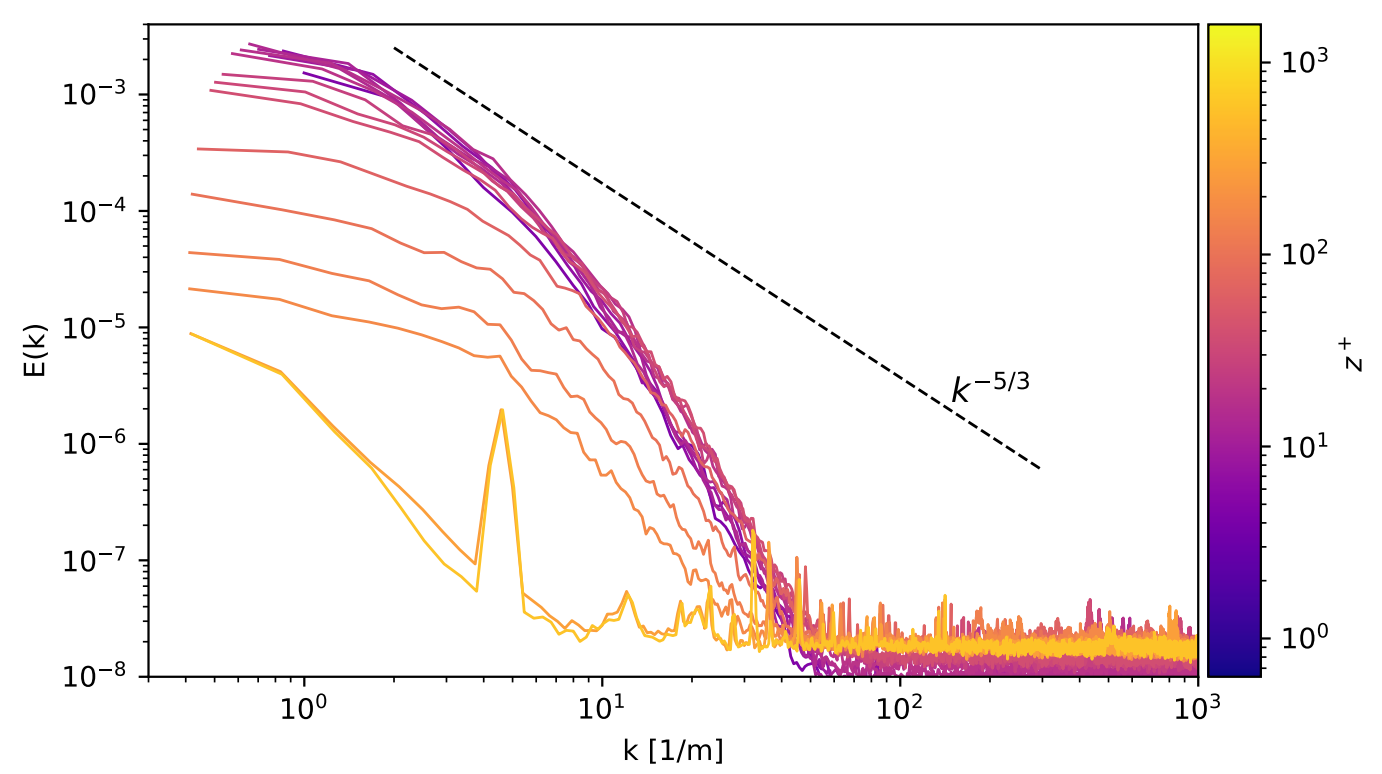

Figure 4.11: Power spectral density distribution for $U_{0}=0.3 \mathrm{~m} / \mathrm{s}\left(R e_{x}=4.7 \times 10^{4}\right)$ and different heights.

$\operatorname{Re}_{x}$. We note that again when we look at the fully turbulent boundary layer the wake region is presented to us with a well-defined peak and a sudden decrease of the $\beta_{4}$ already at the first data-point in the bulk.

The quasi-turbulent boundary layers at the two smallest $\operatorname{Re}_{x}\left(\leq 1.88 \times 10^{5}\right)$ show similar values of $\beta_{4}$, with difference only in the outer region of the boundary layer. There, $\beta_{4}$ does not become positive as for the turbulent boundary layers. This agrees with the observations above that with decreasing $\operatorname{Re}_{x}$ the wake becomes less pronounced. This is because the entrainment of fast fluid from the bulk into the slow boundary layer by large scale eddies does not happen in the transitional state. There, the boundary layer is only intermittently turbulent as we will discuss below.

In fig. 4.11 we present the power spectral density at the smallest bulk flow velocity $U_{0}=0.3 \mathrm{~m} / \mathrm{s}\left(\operatorname{Re}_{x}=4.7 \times 10^{4}\right)$ and different $z^{+}$. This figure should be compared with the energy spectra for the largest $\operatorname{Re}_{x}$ presented in fig. 4.4. In contrast to what was seen for the fully turbulent boundary layer in this regime there is no $z^{+}$range at which a $k^{-5 / 3}$ scaling can be found. Instead, the energy spectrum is mainly shaped by viscous dissipation. 


\subsubsection{Probing the transition from laminarity to turbulence using the M-TERA method}

We hope that we have offered enough and satisfying arguments that the two data-sets with the smallest $\operatorname{Re}_{x}$ fall in the quasi-turbulent or transitional regime. The presented study gives a good idea of what characterizes the boundary layer when we move from a transitional to a fully turbulent regime but unfortunately does not provide clear information about how far we are in the transition. How close are we to the turbulent or the laminar regime?

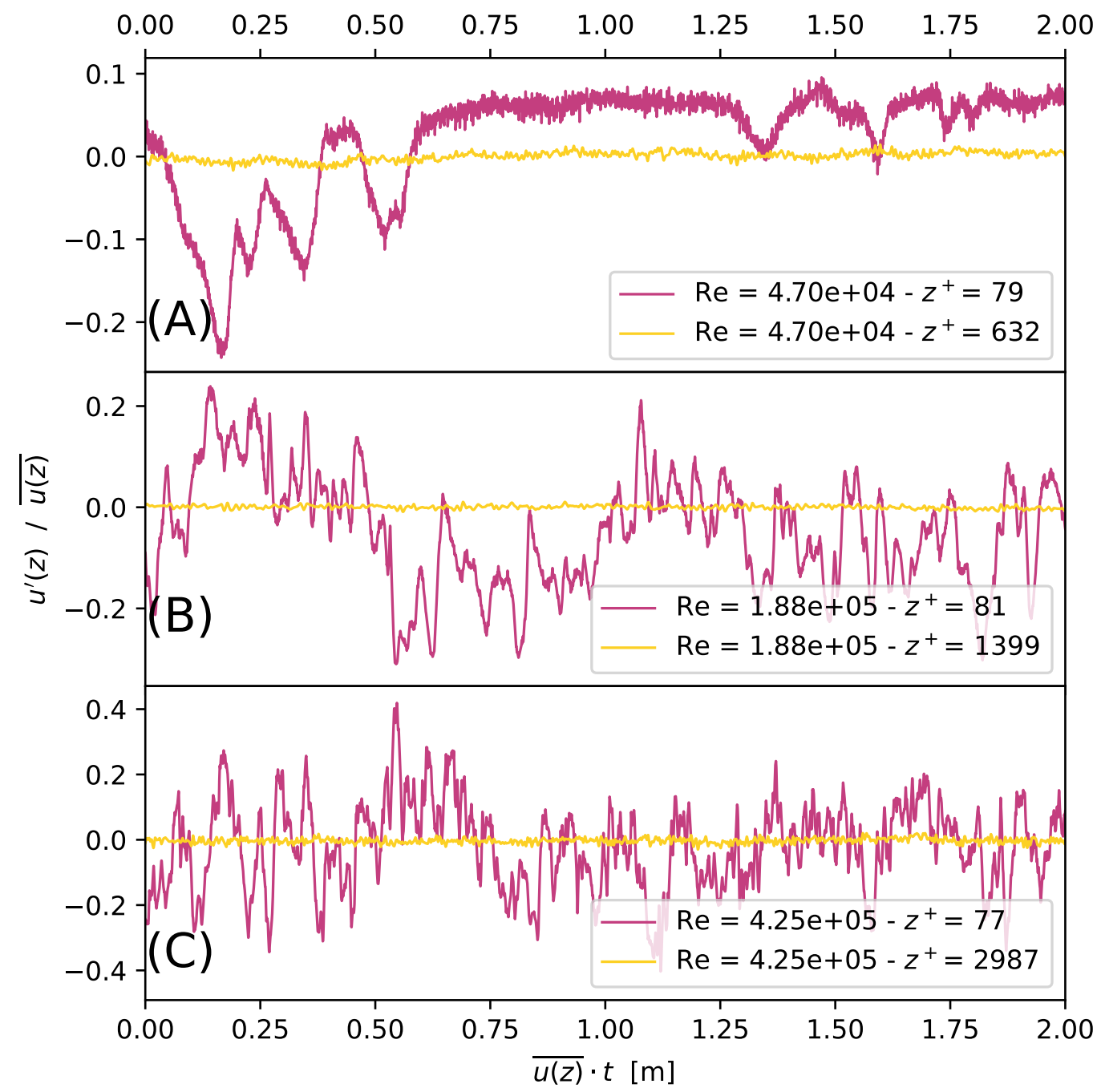

Figure 4.12: Velocity fluctuations normalized as function of the space for: transitional, quasiturbulent and fully turbulent boundary layer. The purple line are data acquired at $z^{+} \approx 80$. The yellow line are data that are collected in the quasi-laminar bulk

While the transition from laminar to turbulent is continuous, it is often desirable to 
have an order parameter that changes with increasing Re from zero in the laminar regime to unity for the turbulent regime and hence characterises the transition quantitatively. With the statistical quantities presented up to now, it is hard to quantify how far the boundary layer is in the transition at any given point.

In fig. 4.12 we show a the normalised velocity fluctuations for a short period, as function of the streamwise distance, with measurements done inside the boundary layer and in the bulk. Data collected inside the boundary layer were taken at $z^{+} \approx 70$, the bulk measurement has been conduced at a surface distance $z=0.4 \mathrm{~m}$ in real units. The free flow velocities of those data are: (A) $U_{0}=0.3 \mathrm{~m} / \mathrm{s},(\mathrm{B}) U_{0}=1.2 \mathrm{~m} / \mathrm{s}$, and (C) $U_{0}=2.71 \mathrm{~m} / \mathrm{s}$.

The transitional boundary layer regime is shown in fig.4.12 (A), the quasi turbulent regime is shown in fig.4.12 (B), the fully turbulent regime is shown in fig.4.12(C). In this we can observe how the transition from laminar to turbulent is characterized as already suggested in sec. 2.3 .3 by an increase of the turbulent intermittency. It is possible to describe the transitional regime as a laminar flow disturbed by sudden turbulent puffs [75]. These puffs are not regular and therefore cannot be statistically characterized by a length or a time interval between them. Nevertheless, it is possible to correlate how often and long are those turbulent puff events in a given time, as an indicator of the turbulent transition. When these turbulent puffs are so frequent that is not possible to distinguish between them anymore forming a long unique turbulent mode, we can say that we are in a fully turbulent regime.

In fig. 4.12 it is clearly possible to distinguish those puffs by naked eye in the transitional flow for the smallest $\operatorname{Re}_{x}$ (fig.4.12 A), where the laminar portions of the velocity are characterized by negligible fluctuations that are comparable with the one in the laminar bulk flow. The sudden spikes in the time represent the turbulent puffs that disturb the flow. In fig. 4.12 (B) where the flow is already quasi-turbulent we do observe an increase of these puffs that are to the naked eye a continuous perturbation, and finally in fig. 4.12 (C) the flow is fully turbulent and hence fluctuation velocity $u^{\prime}$ is never zero for a significant amount of time. This observation can be quantified by introducing a turbulence intermittency factor $\gamma$ that represents the probability to find a turbulent puff at a given point in time. There are different methods to calculate such an intermittency factor. All of them usually consider statistical quantities that are averaged over small time interval, 
which are much smaller than the total duration of the measurement but large enough to gain good statistic for a given interval. For example, one could compare the velocity standard deviation calculated over a given time interval $u_{s t d}$ with the streamwise velocity averaged over the entire measurements $\bar{u}$. One could then assume to detect a turbulent puff, whenever $u_{s t d}>C \bar{u}$. This method is often referred as the $\bar{u}$ method.

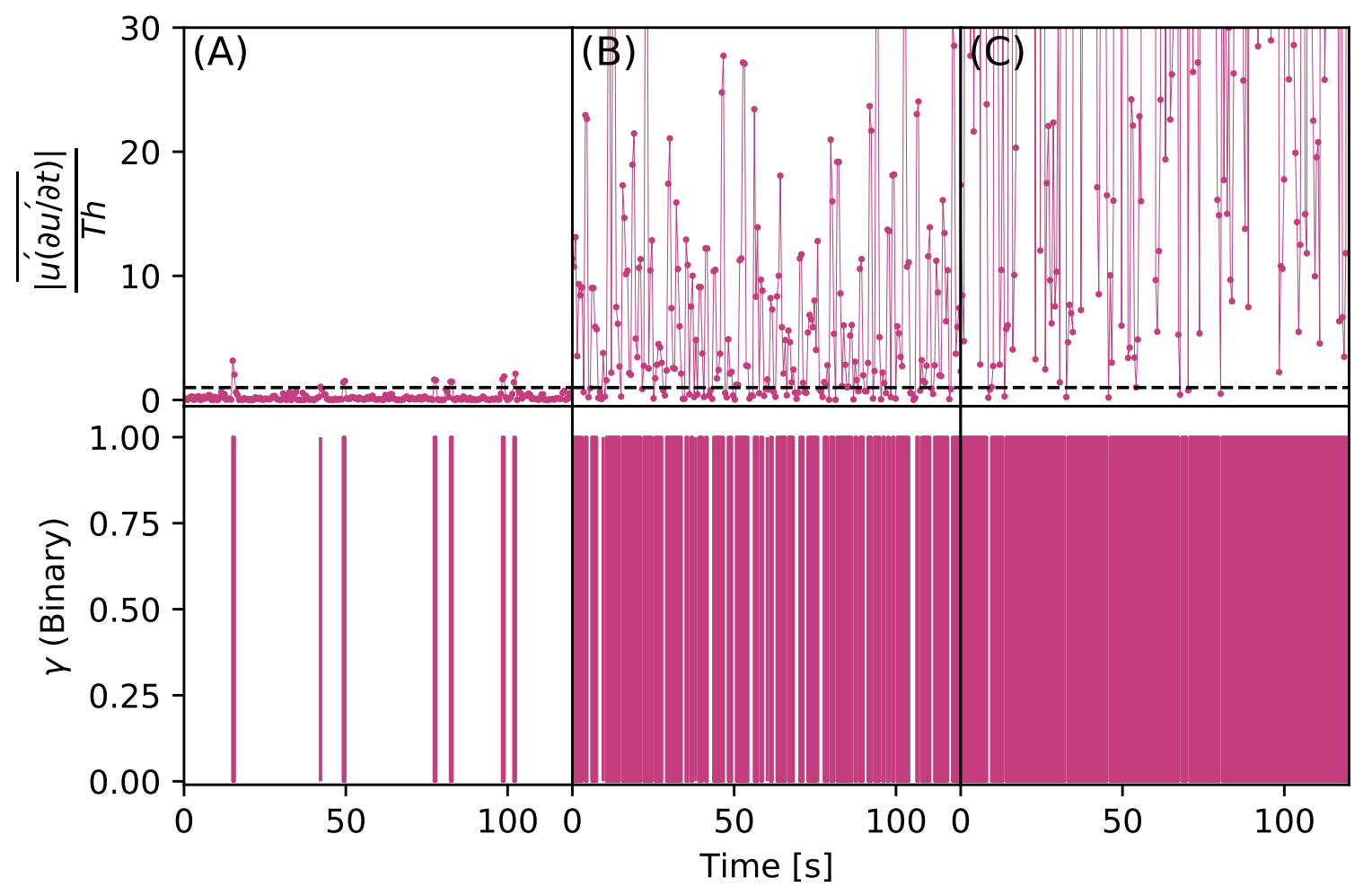

Figure 4.13: Example of thresholds method applied to data-set for different bulk velocities. All the time-series shown in this image are collected at $z^{+} \approx 80$ with (A) $R e_{x}=4.7 \times 10^{4}$ and $U_{0}=0.3 \mathrm{~m} / \mathrm{s}$, (B) $R e_{x}=1.88 \times 10^{5}$ and $U_{0}=1.2 \mathrm{~m} / \mathrm{s}$, and (C) $R e_{x}=4.25 \times 10^{5}$ and $U_{0}=2.71 \mathrm{~m} / \mathrm{s}$.

In this work want to use a slightly more sophisticated method, which was proposed by Zhang in 1995 [76] and which is called Modified Turbulent Energy Recognition Algorithm (M-TERA). The M-TERA has been shown to give more reasonable results then the $\bar{u}$ method or the original TERA method when applied to transitional boundary layers atop a flat plate[77]. ${ }^{1}$ Most notably it correctly returns small values in the viscose sub-layer and in the outer most region of the boundary layer and the bulk. Furthermore, this method is less susceptible to the choice of the threshold value.

In the M-TERA method we first divide a given time-series in multiple chunks of

\footnotetext{
${ }^{1}$ The TERA uses the same detector function $\overline{\left|u^{\prime} \partial u^{\prime} / \partial t\right|}$ function as M-TERA but a different threshold.
} 
segments of constant duration $\Delta t$. When then compute for each chunk a detector function. Compared to the $\bar{u}$-method the detector function is not just $u_{s t d}$ but rather the absolute value of the time derivative of the turbulent kinetic energy, averaged over the time interval $\Delta t,\left|\overline{u^{\prime} \frac{\partial u^{\prime}}{\partial t}}\right|$ with $u^{\prime}=u-\bar{u}$ for each chunk. We then compare it with a threshold function that returns a value of 1 when the chunk in the analysis does contain enough fluctuations to be considered turbulent or 0 when it is considered laminar. The threshold proposed by the M-TERA method is:

$$
T h=C\left[\bar{u} \frac{\left(\partial u^{\prime} / \partial t\right)_{r m s}}{\left(u^{\prime} \partial u^{\prime} / \partial t\right)_{r m s}}\right]
$$

With $\left(\partial u^{\prime} / \partial t\right)_{r m s}$ and $\left(u^{\prime} \partial u^{\prime} / \partial t\right)_{r m s}$ long time standard deviations and C arbitrary constant used to tune the amplitude of the threshold. The value of $\mathrm{C}$ and the time step for the data chunk have to be tuned to the needing and be found according to the specific experimental setup needing. Note that $\mathrm{C}$ is not a dimensionless quantity but has the same units as the threshold, namely $\mathrm{m}^{2} / \mathrm{s}^{3}$. For our experiment we have found the ideal $\Delta t=0.005 s$ and the ideal value of $C=2 \times 10^{-2} \mathrm{~m}^{2} / \mathrm{s}^{3}$. In order to compare our datasets at different velocities it is important to keep $C$ and $\Delta t$ equal. In the optimization of those parameters we attempted to have a non zero $\gamma$ inside the boundary layer for $\operatorname{Re}_{x}=4.7 \times 10^{4}$ and a lower value of gamma for the bulk flow for the higher values of $\operatorname{Re}_{x}$. These values work well in our case to distinguish turbulent from laminar flow patches.

Figure 4.13 shows the entire $120 \mathrm{~s}$ long velocity measurements for the same conditions as previously presented in fig. 4.12. The plot is shown to help the reader to understand the method and has been normalized so that the threshold (dotted line) is at the same height for all three $\mathrm{Re}_{x}$. It has been obtained using eq. 4.6. The top row in fig. 4.13 shows the absolute changes of the kinetic energy averaged over small time segment $(\Delta t)$, i.e., $\left|u^{\prime}\left(\partial u^{\prime} / \partial t\right)\right|$. Every point above the threshold line represents the turbulent flow sections, and the ones that falls below represent the laminar sections. At the bottom in fig. 4.13, we present the binary conversion of the time series presented in fig. 4.13 top. The zeros represent the laminar chunks and the 1 represent the turbulent ones. The final $\langle\gamma\rangle_{t}$ represents the time average of $\gamma(t)$ shown in fig. 4.13 bottom.

Figure 4.14 shows results of the application of the M-TERA method to the boundary layer measurements. As already mentioned, the choice of the threshold value is a bit 


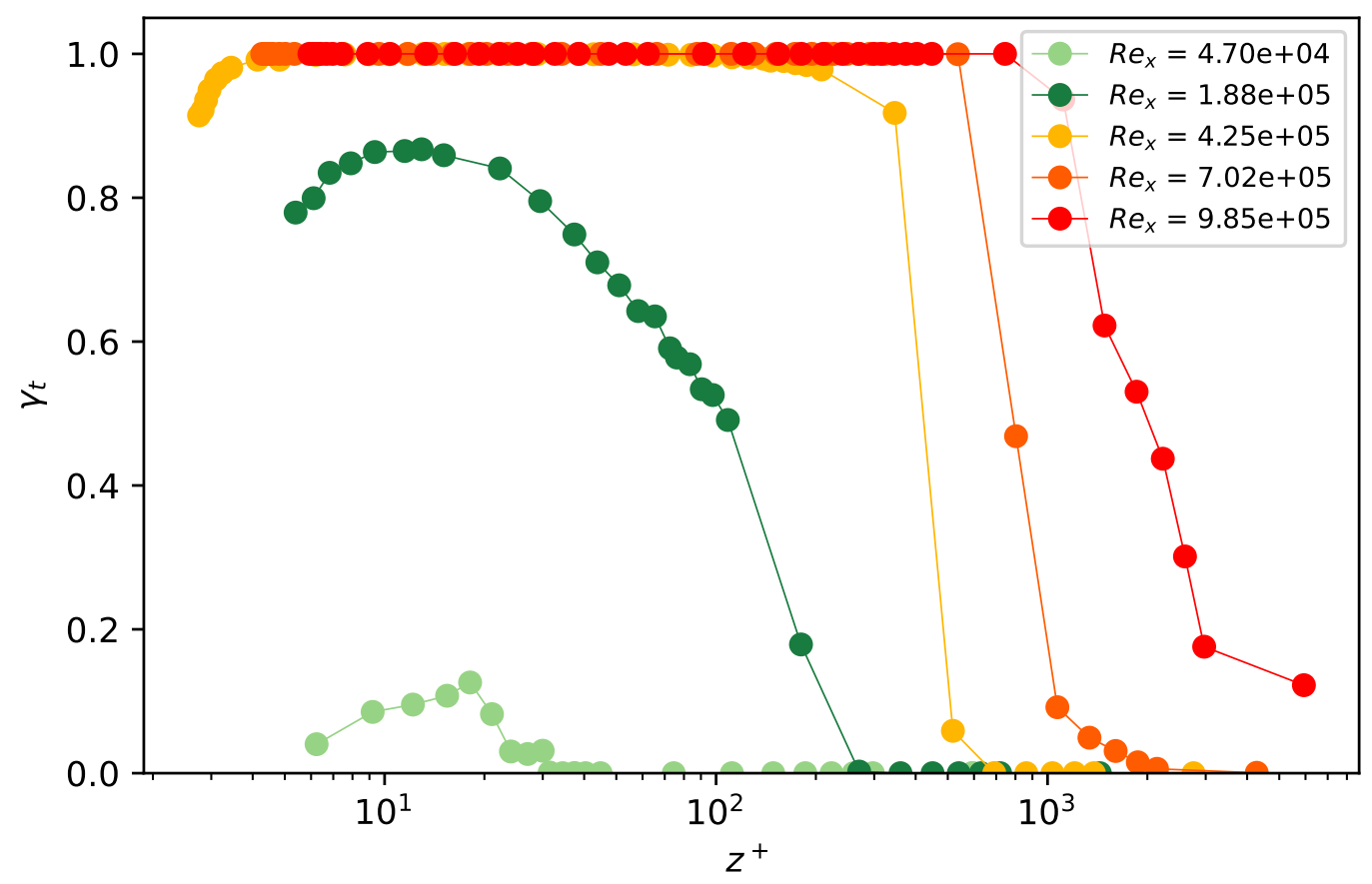

Figure 4.14: Turbulence intermittency as function of velocity and height for $C=7 \times 10^{-9} \mathrm{~m}^{2} / \mathrm{s}^{3}$.

arbitrary and therefore we will discuss our finding and observation with the threshold of our choice.

Our observations for the turbulent intermittency $\langle\gamma\rangle_{t}$ confirm what has been suggested at the beginning of the section. The fully turbulent boundary layers with $R e_{x}>1.88 \times 10^{5}$ are presented with $\langle\gamma\rangle_{t}=1$ along the boundary layer and a decrease in the wake towards the bulk region. An increase in the bulk flow speed introduces some turbulence in the bulk flow that leads to finite $\langle\gamma\rangle_{t}$ there. At $R e_{x}=1.88 \times 10^{5}$ the flow is quasi-turbulent as was expected before, with $\langle\gamma\rangle_{t}$ getting close to one inside the boundary layer but never reaching it. For $R e_{x}=4.7 \times 10^{4},\langle\gamma\rangle_{t}$ never surpasses the value of 0.2 .

Our result shows that both data-sets collected with lowest shear stress are not in a fully turbulent regime, with one being clearly close to the full turbulent transition and the other rather close to laminarity. To better quantify the transition we have choose to present an average of all the $\langle\gamma\rangle_{s}$ inside the boundary layer, with boundary layer size $z<\delta_{99}$ and present the results in table 4.2 : 


\begin{tabular}{||c|c||}
\hline$R e_{x}$ & $\langle\gamma\rangle_{s}$ \\
\hline $4.70 \times 10^{4}$ & 0.037 \\
\hline $1.88 \times 10^{5}$ & 0.662 \\
\hline $4.25 \times 10^{5}$ & 0.957 \\
\hline $7.02 \times 10^{5}$ & 0.984 \\
\hline $9.85 \times 10^{5}$ & 0.998 \\
\hline
\end{tabular}

Table 4.2: Experimentally determined turbulence intermittency at various free flow velocities. $\langle\gamma\rangle_{s}$ represents the average of the gamma for $z<\delta_{99}$

\subsection{Velocity boundary layer with heating}

In the previous sections, our analysis has focused on the classical wall-bounded flow above an adiabatic plate, with only the free flow velocity $U_{0}$ as control parameter. In this section, we will heat the surface of the wall to a temperature $T_{w}$ that is larger than the air temperature of the incoming flow $T_{0}$. The combination of these two control parameters allow us to study the influence of buoyancy on the flow inside the boundary layer and we can make measurements in the regime of forced convection, where buoyancy is negligible and the mixed convection regime, where buoyancy becomes significant. Note that we are not really able to make measurements in the regime where buoyancy dominates over the shear forces (mixed convection) since the flow needs to be fast enough to carry the thermal plumes away before they reach the top of the wind-tunnel. Furthermore, the velocity probes can only measure flow speeds reliably for sufficiently fast flows so that buoyancy created by the hot-wire probe themselves is negligible for the cooling of the probe.

\subsubsection{Velocity boundary layer in turbulent regime with heated surface}

Now, we will investigate the interaction between the temperature and velocity field focusing on the velocity field by comparing how the statistical flow quantities analysed above in sec. 4.1 change with increasing wall temperature. The Grashof number (Gr), already introduced in section 2.4.2, allows us to express the surface temperature as a dimensionless control parameter.

The buoyant forces are directly related to the surface temperature and therefore $G r$ 
is defined as:

$$
G r=\frac{g \beta \Delta T L^{3}}{\nu^{2}}
$$

with $\Delta T=T_{w}-T_{0}$ being the temperature difference between the local surface temperature at $x=2.35 \mathrm{~m}$ and the temperature of the incoming bulk flow. Since we cannot measure the skin friction velocity $u_{\tau}$ directly, and since we do not know how the velocity profile changes with heating, we normalise our data using $u_{\tau}$ determined without surface heating applied and listed in table 4.1. This method will allow us to directly compare changes of the velocity profile introduced by the surface temperature along the boundary layer.

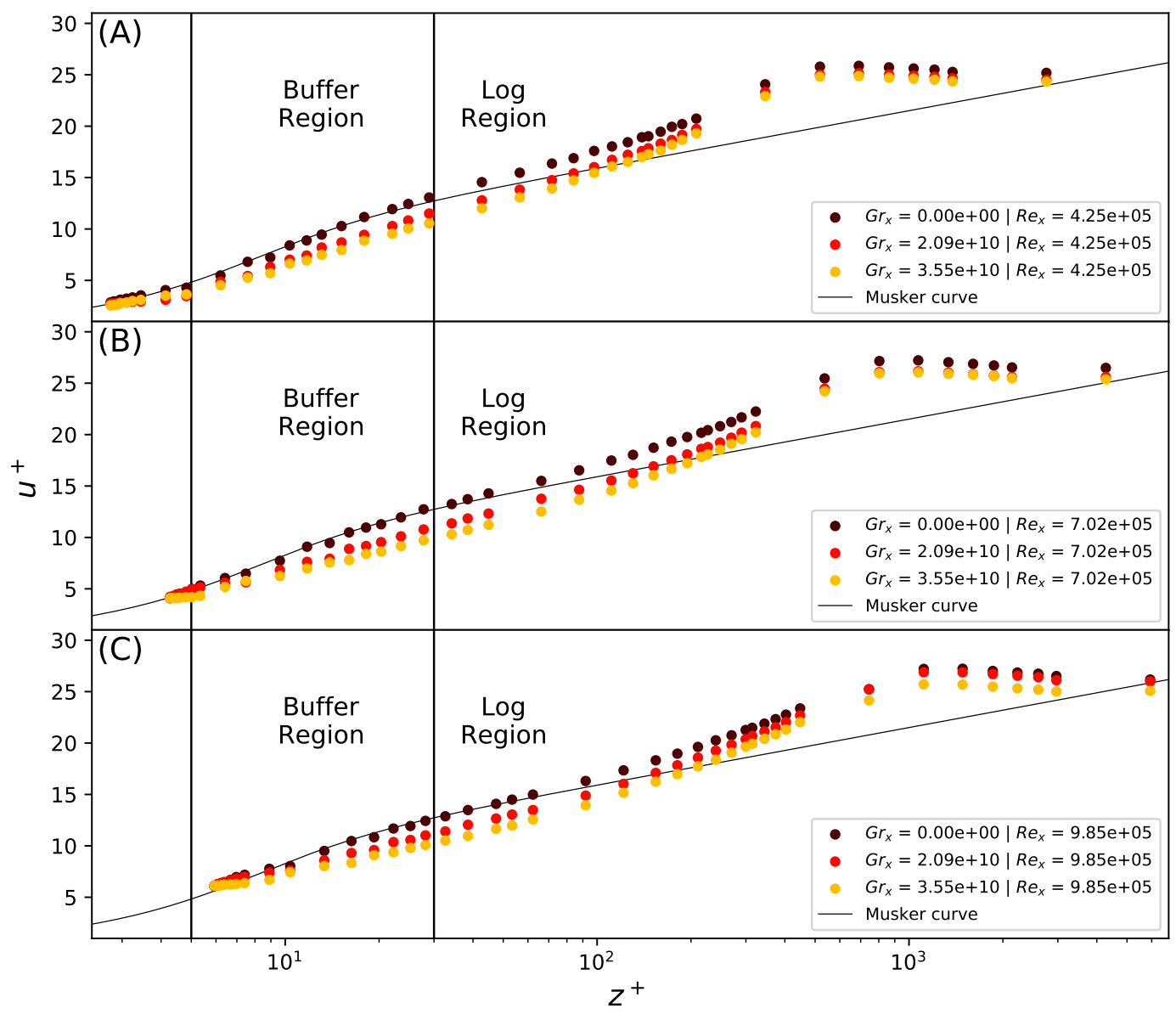

Figure 4.15: Average velocity as function of distance to the plate for the three largest $R e_{x}$ where the boundary layer is fully turbulent and for different surface temperatures.

In fig. 4.15 the velocity profile is presented as function of various surface temperature. When extremely close to the surface, the velocity profiles show to be coincident for all the surface temperature. Moving towards higher altitudes we do observe a decrease of 
the average velocity in the buffer region $z^{+} \approx 15$, for higher surface temperature. This velocity difference propagate along the boundary layer up to the wake region, where the velocity average seems to converge again with values closer to the non-heated case. One could expected that heating the surface would mostly affect the near wall regions as there the temperature gradient and hence buoyancy is strongest.

As introduced in sec. 2.3.2 the buffer region represents the transition region between viscous sub-layer and log region at which both viscosity and advection contributes to momentum transport. This dual nature results in a continuous transition between those two regimes and can be recognized by the characteristic bump that can be observed in our velocity profiles when $G r_{x}=0$. When the heat is introduced, the equilibrium between this two momentum transport mechanism shifts. With increasing surface temperature the buffer region moves towards a more log-like profile. We can interpret this as a weaker viscous momentum diffusion against a stronger advective momentum one.

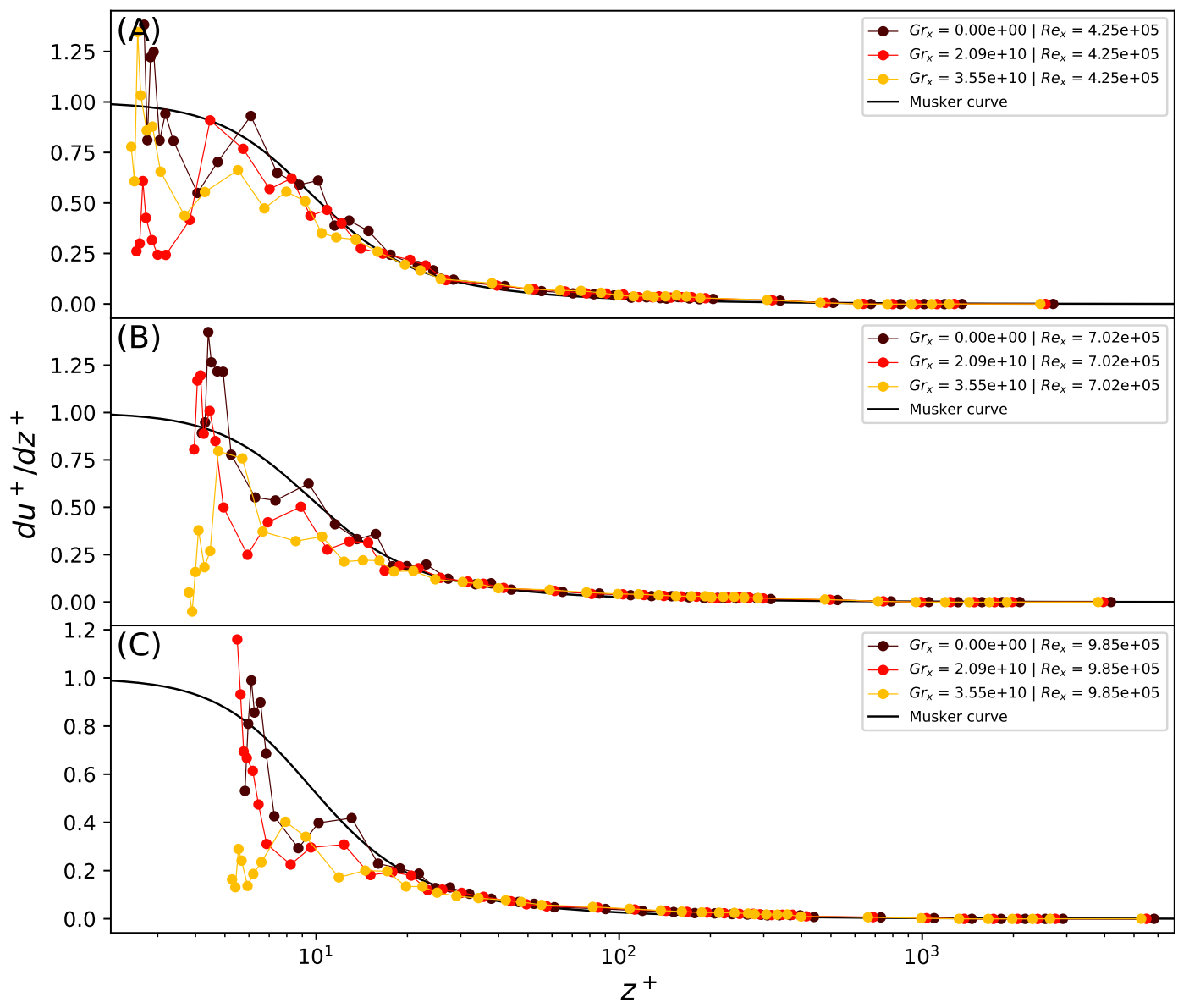

Figure 4.16: Averaged velocity gradient above a heated plate for different surface temperatures and at different $R e_{x}$. 
It is common knowledge that the increase of air temperature (our fluid) does results in an increase in kinematic and dynamic viscosity. One could expect that an increase of viscosity would result in an increase of the viscous sub-layer, and also remember the correlation between viscosity and Reynolds number with $R e=\frac{\mathbf{u} L}{\nu}$, where a higher value of viscosity results in a smaller $R e_{x}$, therefore a later transition from laminar to turbulent regime. According to our data this is not the case, from the pure analysis of the viscosity we would expect an opposite effect that what we do observe inside the boundary layer. It is important to present all our data and analysis before drawing any conclusion on the reasons for this observation.

In fig. 4.16 we present an analysis of the wall normal velocity gradient as function of the vertical position.

It is possible to connect the velocity gradient with the local momentum transport. In general, momentum is transported from the wall to the bulk flow over the boundary layer. The momentum is transported on one hand by viscose shear and on the other hand via advection with the flow. Because the transport is in wall normal and streamwise direction, the wall normal transport is not constant in $\mathrm{z}$-direction at a given point $x$.

However, since the average velocities $\left\langle u^{+}\right\rangle$change only little with increasing Gr, we can link changes in the local velocity gradient $\partial u^{+} / \partial x^{+}$to changes in the momentum transport, which is done mostly by viscosity in the sub-layer and by eddy viscosity above. Therefore, a smaller gradient would correspond to a larger momentum flux in wall normal direction.

Having this said, we observe a decrease in the momentum flux very close to the surface when heat is applied. A decrease of the momentum transport should also lead to an increases of the boundary layer height. In our experiment this does not seem to be the case, although we do not have many measurement points in the upper part of the boundary layer and hence only limited spatial resolution there.

Figure 4.17 shows the normalised velocity standard deviation for the same $G r_{x}$ and $R e y_{x}$ presented in fig. 4.15. Here, we can highlight some similarities in the regions mainly affected by the introduction of an heated surface. When the physical distance from the wall is minimal not only the velocity, but also the $u_{s t d} / u_{\tau}$ coincides. It is hence worth noting that also the velocity fluctuations converge for different temperatures at the same distance from the plate. This distance from the plate is at all the various $\operatorname{Re}_{x}$ equal to 


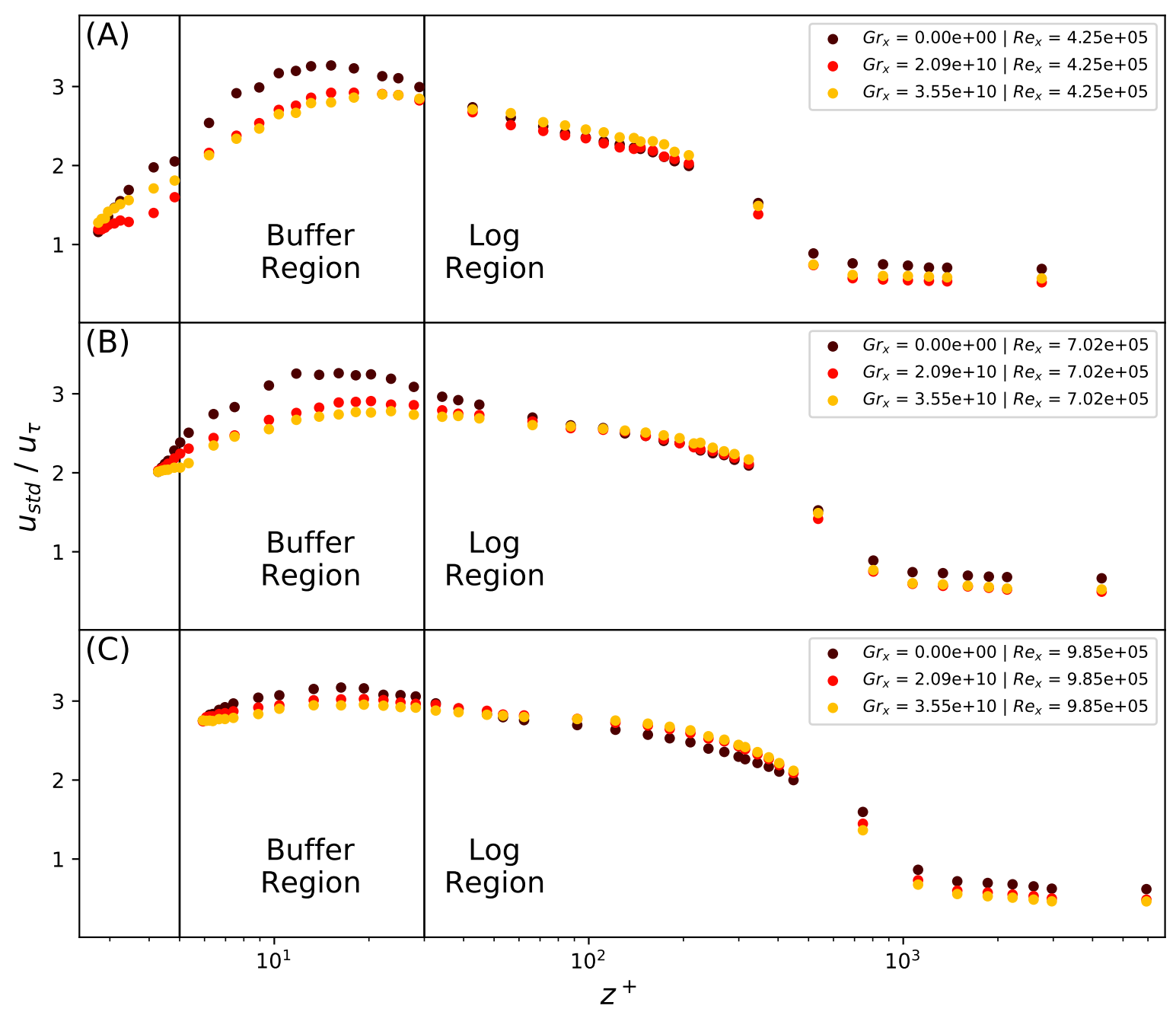

Figure 4.17: Standard deviation in fully turbulent boundary layer at different surface temperatures. 
$z \approx 0.4 \mathrm{~mm}$ in real units.

As already observed in fig. 4.15 and fig 4.17, the main effects of the increased surface heat can be observed in the buffer region for $z^{+}<30$. When the $\operatorname{Re}_{x}$ is smaller, the changes in $u_{s t d} / u_{\tau}$ due to increasing $\mathrm{Gr}_{x}$ are stronger. As a result, the maximum in the buffer region significantly decreases, smoothing the transition between the buffer and the $\log$ region. While this effect is stronger for smaller $\mathrm{Re}_{x}$, it is also observed for the largest $\operatorname{Re}_{x}=9.85 \times 10^{5}$. Further minor effects of the surface heat can be observed in the wake region, where the temperature increase causes a weak increase of the $u_{s t d} / u_{\tau}$. We believe that this effect is related to the steeper velocity gradient, which results in stronger shear stress and hence slightly higher turbulent intensity.

\section{The distribution of velocity fluctuation}

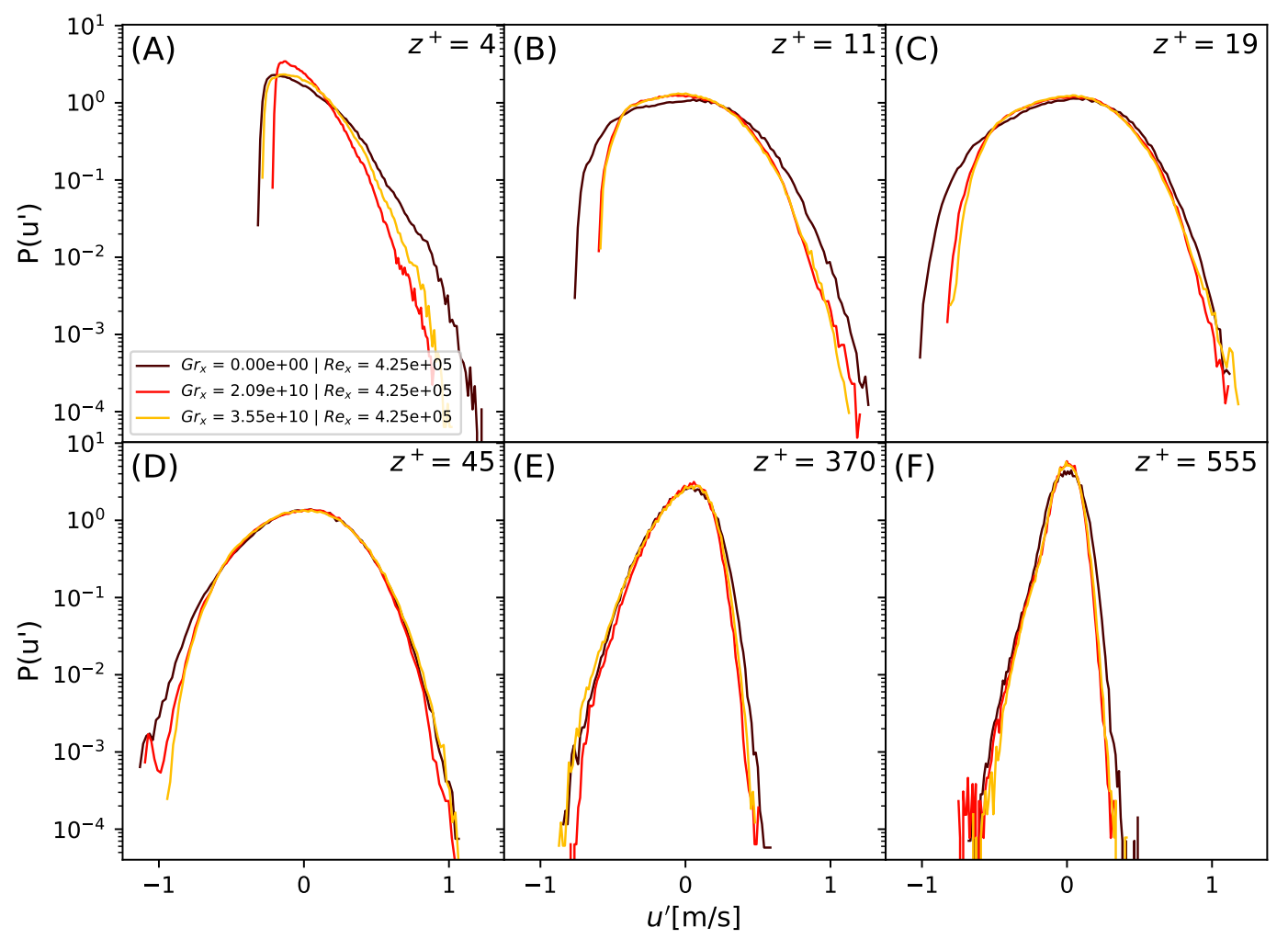

Figure 4.18: Probability density function of velocity fluctuations for different surface temperature and Reynolds number $R e_{x}=4.25 \times 10^{5}$.

Figure 4.18 shows the probability density function for $R e_{x}=4.25 \times 10^{5}$ and three different $\mathrm{Gr}_{x}$. For this case, the boundary layer was still fully turbulent but the effect of a heated wall was strongest among the $\mathrm{Re}_{x}$ measurements with fully turbulent boundary 
layer. Small changes with increasing $\mathrm{Gr}_{x}$ can only be seen for the three measurements closest to the wall. We see again that with increasing $\mathrm{Gr}_{x}$, the probability density functions become narrower and their maxima shifts to larger velocities, which is in accordance to observations made in fig. 4.15 and 4.17. From fig. 4.18 it is difficult to judge by eye, how other quantities of the velocity distribution changes. Therefore, we consider other statistical quantities, namely the skewness and kurtosis.

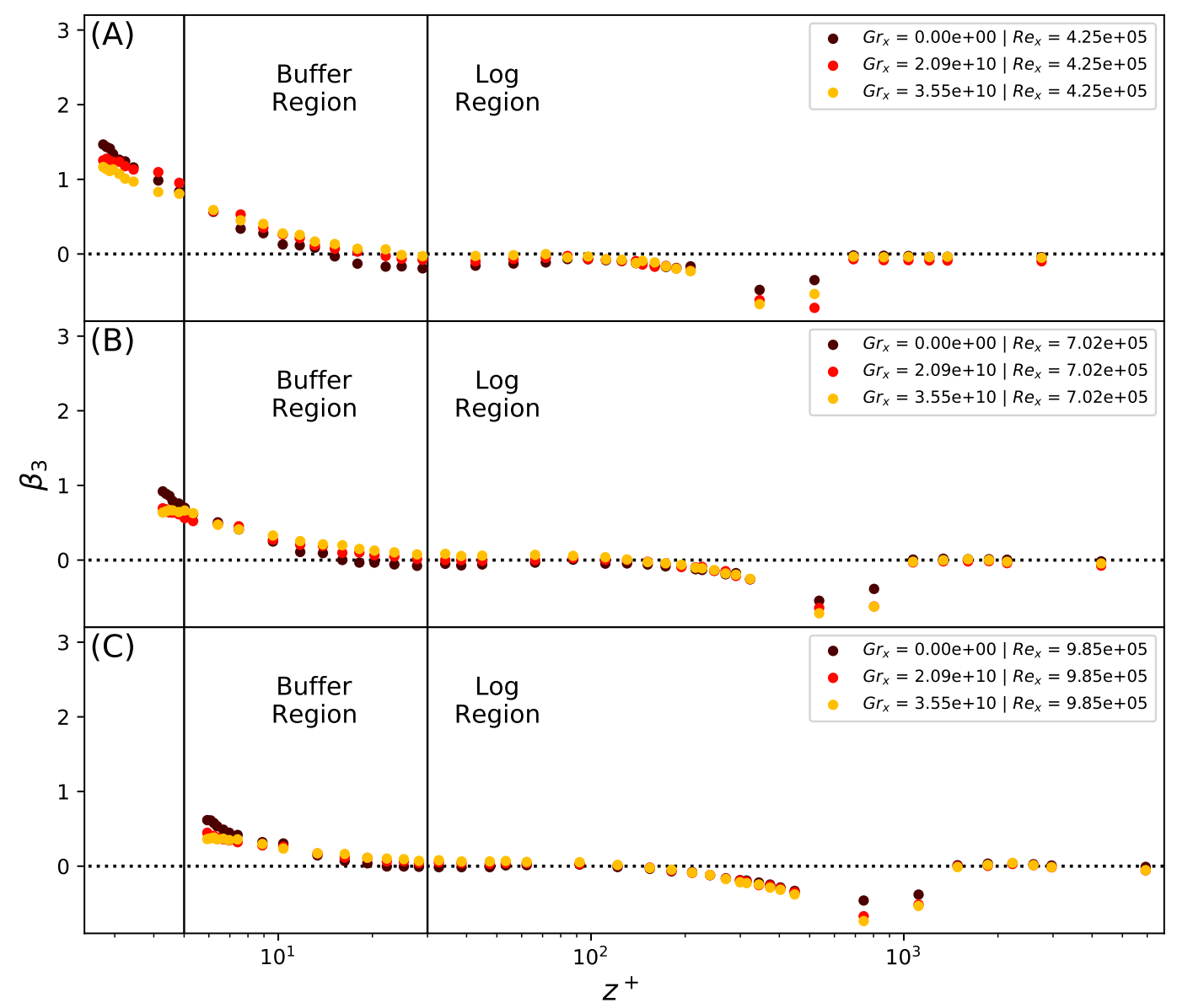

Figure 4.19: Skewness in fully turbulent boundary layer at different surface temperatures

The skewness $\beta_{3}$ of the same data-sets is shown in fig. 4.19. It does not highlight any strong difference between heat and unheated surface. We observe some minor changes induced by the heat, but altogether they seem to be mild in the total scheme of things. The region very close to the surface shows slightly lower values of $\beta_{3}$ with increasing $\mathrm{Gr}_{x}$, and slightly higher value of $\beta_{3}$ in the buffer region. Also, the wake is weakly affected by these variations resulting in a more skewed distribution. The general feeling is that the steeper wake velocity gradient does affect his skewness resulting in a slightly more 
asymmetrical transition.

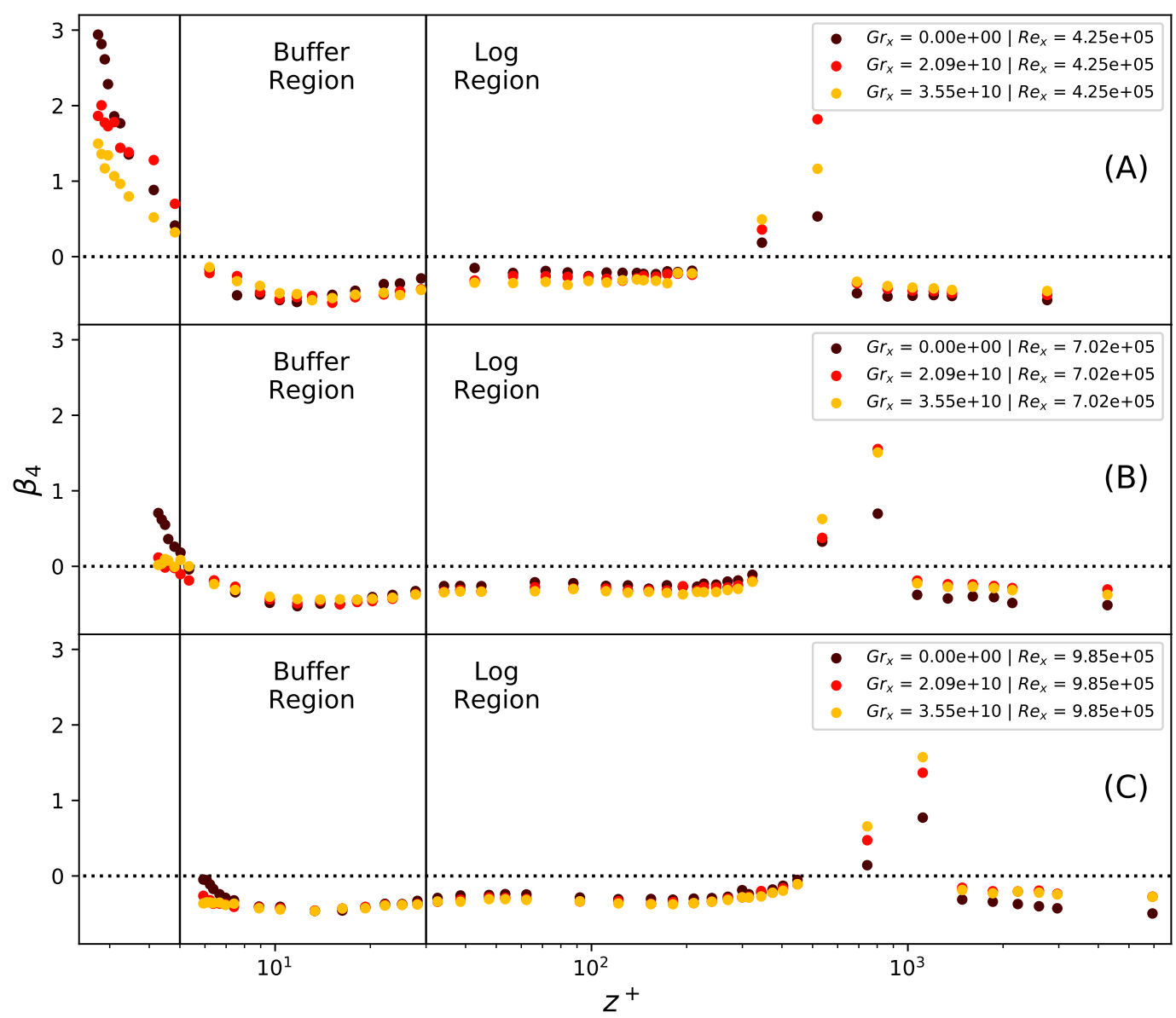

Figure 4.20: Kurtosis in fully turbulent boundary layer at different surface temperatures.

The kurtosis is shown in fig. 4.20, again for the same data-set as before. Very close to the wall, we can see decrease of $\beta_{4}$ when $\mathrm{Gr}_{x}$ increases. This effect is stronger for smaller $\mathrm{Re}_{x}$. This observation is in somehow in contrast to fig. 4.15, where the average velocity converged for different $\mathrm{Gr}_{x}$ towards a common value close to the surface. This hints that, despite not being visible in the average velocity, the heat does have some effect on the boundary layer flow at the wall proximity. This results with increasing temperature in a velocity data-set more prone to present extreme events that what is observed without heat applied. We also observe a second region of the $\beta_{4}$ that is strongly affected by the surface temperature increase, the wake. The wake is affected by the temperature in with an opposite effect respect to what has been observed at the surface proximity, the heat increase results in less extreme events. We are not fully capable of justify this effect, but the increase in temperature decreases the amount of high amplitude perturbations that 
can be observed in the wake.

\section{Power Spectrum}

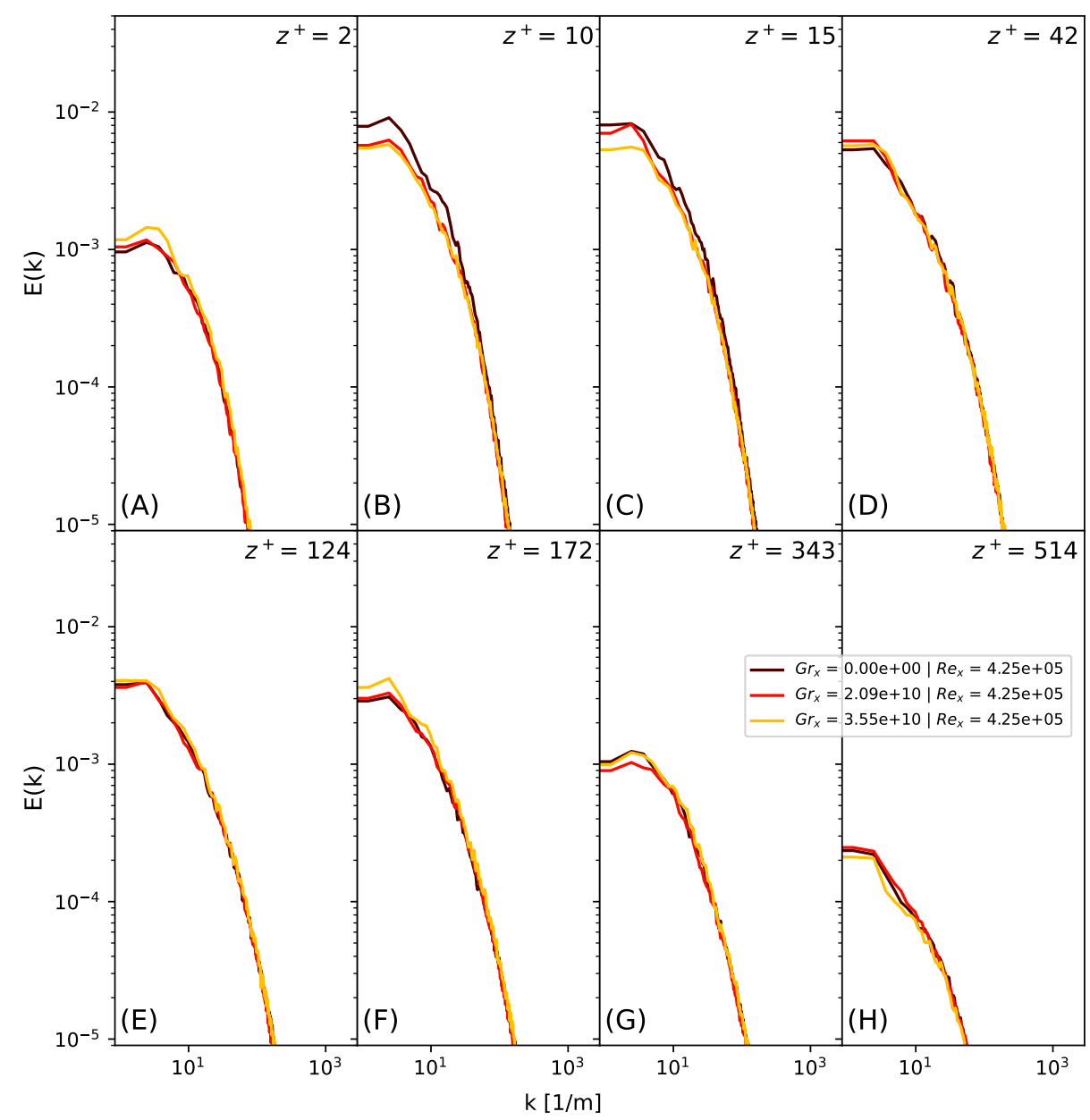

Figure 4.21: Power spectral density analysis for different surface temperatures and flow velocity $U_{0}=2.71 \mathrm{~m} / \mathrm{s}\left(\operatorname{Re}_{x}=4.25 \times 10^{5}\right)$.

Figure 4.21 shows the comparison of the power spectra for different vertical positions and different $\operatorname{Gr}_{x}$ at $U_{0}=2.71 \mathrm{~m} / \mathrm{s}\left(\operatorname{Re}_{x}=4.25 \times 10^{5}\right)$. In this and other power spectra in fully turbulent flows, there is no significant $\mathrm{Gr}_{x}$ dependency that qualitatively affects the data. The eddy energy cascade seems to be unchanged when heated from below.

However, by fitting eq. 4.5 to the data we can detect the $k^{-1}$ and $k^{-5 / 3}$ ranges as well as the wave number $k_{t}$ where one ends and the other start as discussed in sec. 4.1.

For the sake of brevity we present in fig. 4.22 only the fitted transitional wavenumbers $k_{t}$ together with lines that represent fits to the power law $k_{t} \propto z^{+\beta}$ for these points. The exponents for the three different $\mathrm{Gr}_{x}$ are listed in tab. 4.3: 


\begin{tabular}{||ccc||}
\hline$R e_{x}$ & $G r_{x}$ & $\beta$ \\
\hline $9.85 \times 10^{5}$ & 0 & -0.41 \\
\hline $9.85 \times 10^{5}$ & $2.09 \times 10^{10}$ & -0.46 \\
\hline $9.85 \times 10^{5}$ & $3.55 \times 10^{10}$ & -0.48 \\
\hline
\end{tabular}

Table 4.3: Fitting parameter used to obtain figure 4.22

From our observations, it seems that the surface temperature has a slight effect on $k_{t}$, causing it to increase slightly with increasing $\mathrm{Gr}_{x}$. As expected, this effect is mostly visible at lower $z^{+}$with coincident results in the high $z^{+}$region despite the temperature increase. If we recall the explanation for the $k^{-1}$ decrease (see sec. 2.3.2) we could speculate on the effect of the heat on the energy spectrum. The $k^{-1}$ regime is suggested to be originated by different horizontal and vertical velocity gradients in the proximity of a surface. It is unclear at this point how heat can change the wave number at which the flow becomes isotropic. One could speculate that the wave number at which energy is introduced from buoyancy is larger than the wave number at which energy is created from the shear. Therefore, the wave number at which isotropy is reinstalled due to the cascade is smaller. The transitional $k_{t}$ can be interpreted as exactly this wave number above which the turbulence is isotropic.

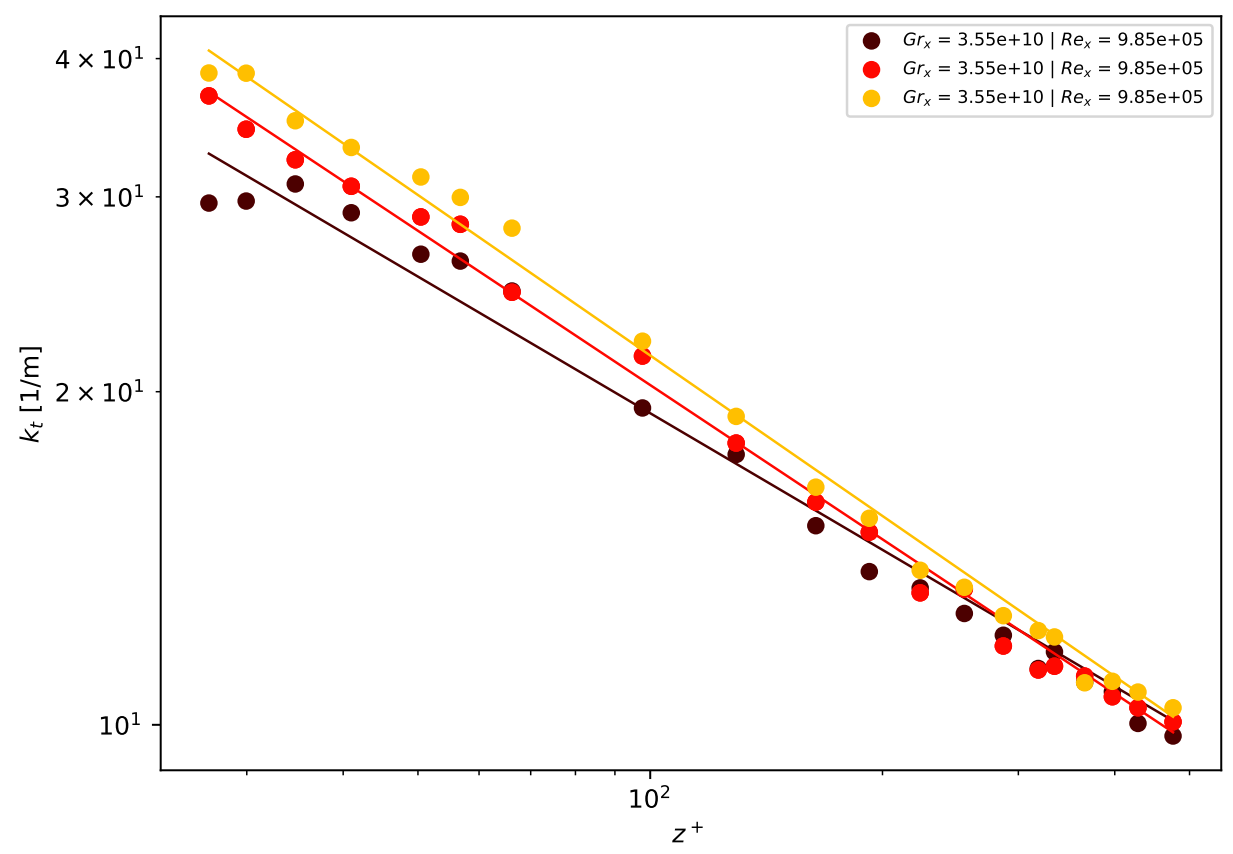

Figure 4.22: $k$-1 transition point for $R e_{x}=9.9 \times 10^{5}$. 
As the introduction of energy from buoyancy is largest close to the wall, also the change of $k_{t}$ with increasing $\mathrm{Gr}_{x}$ is largest there. We do expect this effect to only exist in a small portion of the boundary layer because in the outer regions of the boundary layer, the shear forces is dominant.

\section{Turbulence intermittency}

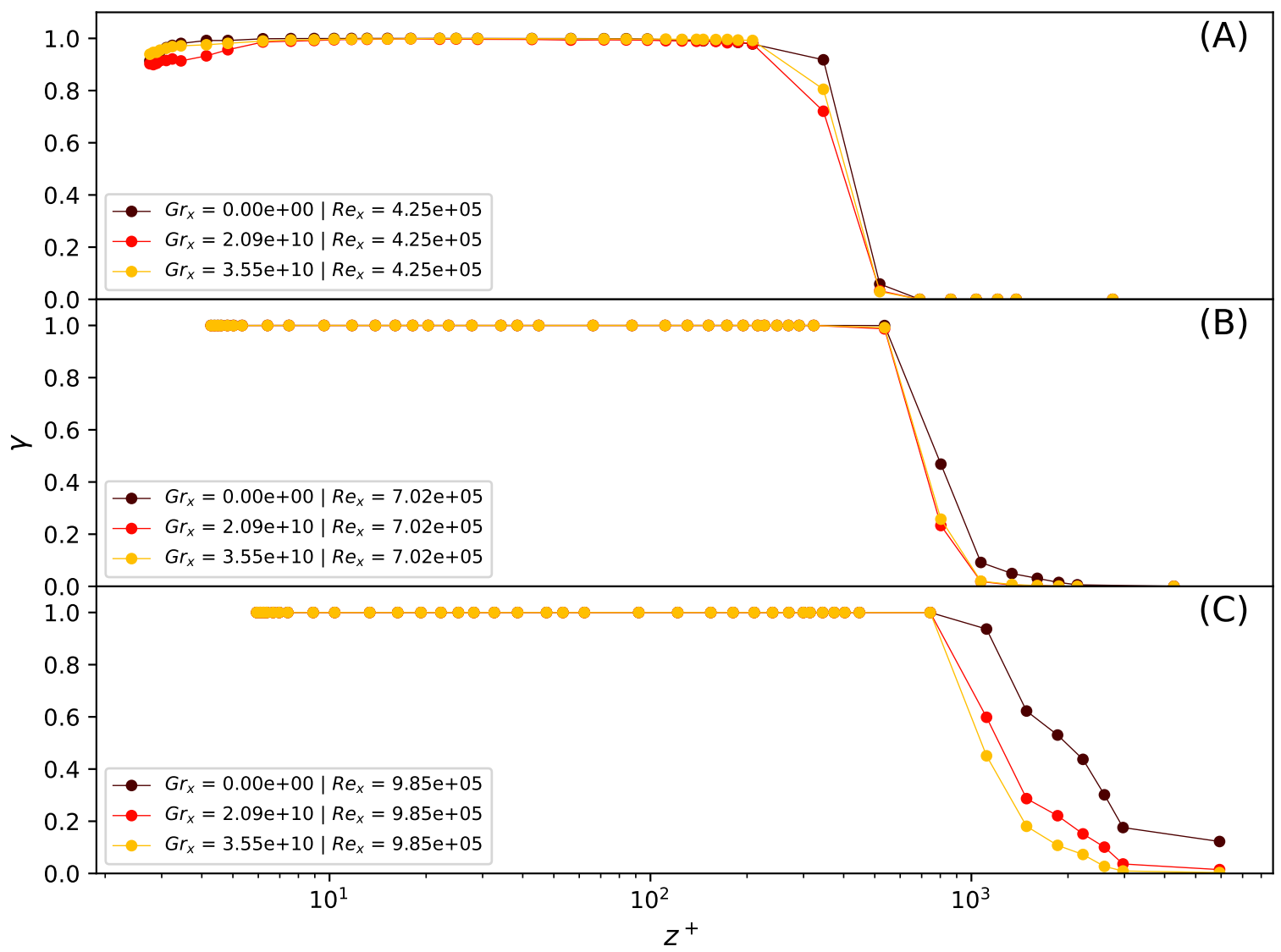

Figure 4.23: Gamma variation for different surface temperature and flow velocities in turbulent boundary layers.

In fig. 4.23 we examine the effect of the surface heating on boundary layer turbulence intermittency along $z^{+}$. The turbulence intermittency $\gamma$ does not change for most of the boundary layer at any given $R e_{x}$, with a decrease only close to the bulk. There, strangely, we do observe lower turbulence intermittency when heat is applied. This observation is surprisingly consistent and seen in fig. 4.23(A, B, and C). It also agrees with slightly reduced standard deviation in the bulk as seen in fig. 4.17. The reason for this is unclear. The flow so far away from the surface is not expected to be influenced directly by the 
boundary conditions. One might speculate about some electrical interferences between the sensitive CTA and the high current of the electrical bottom plate heater. This perturbation can be observed only in the nearly laminar bulk, and we believe it is negligible for measurements inside the boundary layer.

The measured average gamma inside the boundary layer result to be:

\begin{tabular}{||ccc||}
\hline$R e_{x}$ & $G r_{x}$ & $\langle\gamma\rangle$ \\
\hline $4.25 \times 10^{5}$ & 0 & 0.957 \\
\hline $4.25 \times 10^{5}$ & $2.09 \times 10^{10}$ & 0.938 \\
\hline $4.25 \times 10^{5}$ & $3.55 \times 10^{10}$ & 0.952 \\
\hline $7.02 \times 10^{5}$ & 0 & 0.985 \\
\hline $7.02 \times 10^{5}$ & $2.09 \times 10^{10}$ & 0.938 \\
\hline $7.02 \times 10^{5}$ & $3.55 \times 10^{10}$ & 0.978 \\
\hline $9.85 \times 10^{5}$ & 0 & 0.998 \\
\hline $9.85 \times 10^{5}$ & $2.09 \times 10^{10}$ & 0.986 \\
\hline $9.85 \times 10^{5}$ & $3.55 \times 10^{10}$ & 0.984 \\
\hline
\end{tabular}

Table 4.4: Experimentally determined turbulence intermittency at various free flow velocities and surface temperatures. $\langle\gamma\rangle$ represents the average of the gamma for $z<\delta_{99}$

To summarize, in this section we investigated the effect of a heated wall on the velocity field of a fully turbulent boundary layer. At the smallest distance from the surface we have observed no influence of $\mathrm{Gr}_{x}$ on the average velocity or $u_{s t d}$, but we have observed an influence on $\beta_{3}$ and $\beta_{4}$. Moving up in altitude we observed the buffer region to be more influenced by the temperature increase, with a consistent decrease of the average velocity, coupled with a decrease of relative fluctuation in the same region when heat is applied. Velocity variations induced by the heat can also be observed in the log and wake region, where the average velocity is mainly affected, with in particular the wake region acting as a balancing system where the different temperatures flow with different velocities does converges to the free flow velocity keeping the boundary layer size rather unchanged within the resolution of our measurements.

One could argue that the differences in the velocity measurements are due to experimental errors, induced by the wall proximity and the heated fluid around the hot-wire. The hot-wire measurements are by nature susceptible to strong temperature variations in the fluid medium. The extreme wall proximity, and strong changes in temperature in our experiment has already been discussed in sec. 3.3.1. We concluded that the error induced by the increase in temperature would be in the worst case scenario around $3 \%$ 
of the average velocity, far from the values observed in our analysis. Another important element to consider is that an measurement error induced by the heat should result in a lower velocity when the fluid medium is warmer. While this is somehow the case for the average velocity, such an effect should be strongest closer to the wall, which is not observed in fig. 4.15 .

\section{Final thoughts}

We will now try to interpret our observations.

We would like to begin by pointing at similarities between a heated plate and a plate with a rough surface. Jimenez in 2004 [78] reviewed experimental observations on turbulent flows over rough surfaces. In his study, he concluded that: "The classical result is that the buffer layer can be perturbed without transmitting to the outer flow anything beyond a change in skin friction, but there are indications of deeper interactions", and also suggested that an increasing roughness would usually result in an increase of velocity fluctuations in the log region.
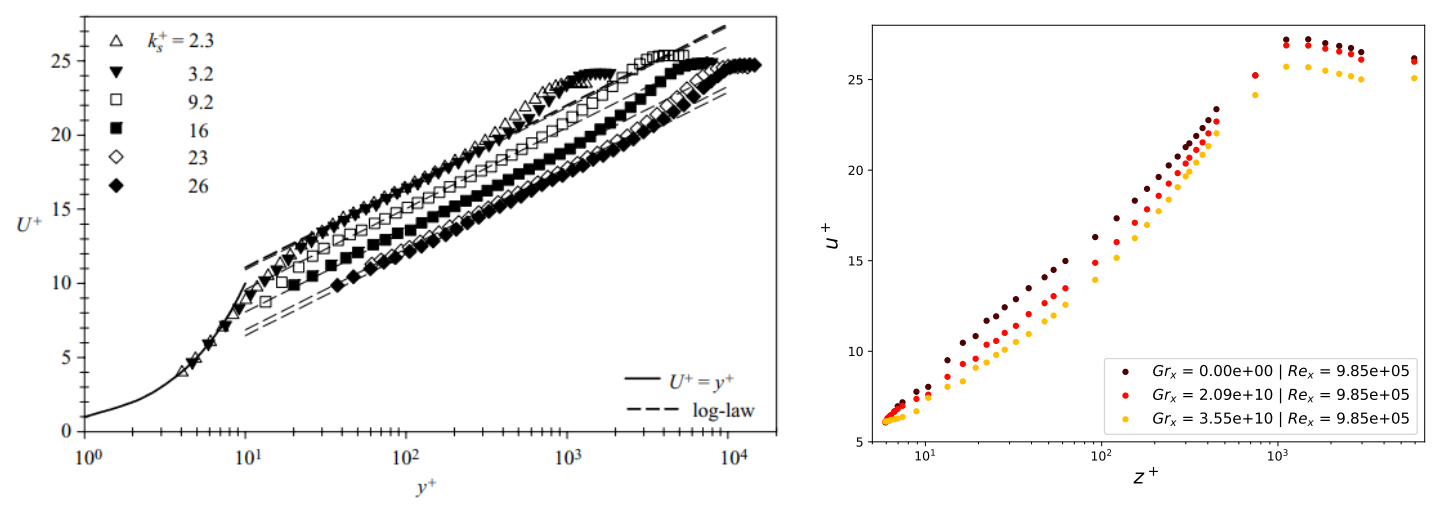

Figure 4.24: Left - Rough-wall mean velocity profiles. $k_{s}^{+}$represents relative roughness. Plot from Schultz 2007 [79]. Right - Data extracted from fig.4.15 for reference

Flack in 2005, Schultz in 2007 and Squire in 2016 ([79-81]) presented experimental investigations that confirm some of the findings from Jimenez [78]. Those studies provide us with some useful data that we can compare to our results. Figure 4.24 is extracted from Schultz [79] and represent the velocity profile for a turbulent boundary layer with increasing wall-roughness. The increase in roughness does not only results in a lower average velocity $u^{+}$, but also shows a gradual reduction of the buffer region, which is replaced by a log-like scaling. Figure 4.25 extracted from Schultz [79] presents the standard 

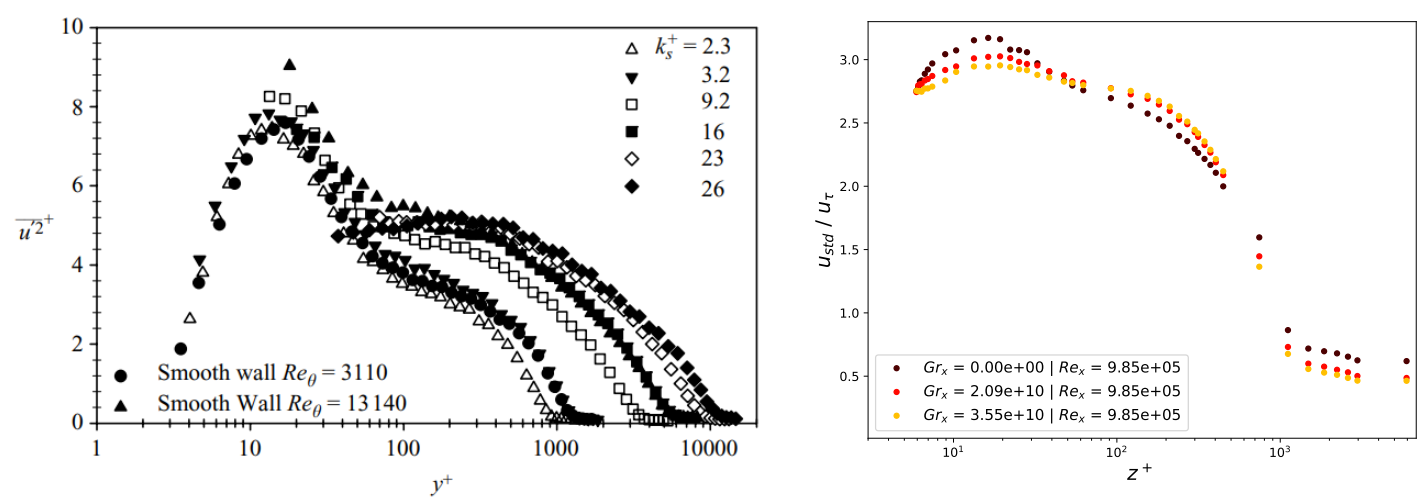

Figure 4.25: Left - Rough-wall standard deviation profiles. $k_{s}^{+}$represents relative roughness. Plot from Schultz 2007 [79]. Right - Data extracted from fig.4.17 for reference

deviation, where we can observe that the increase of roughness results usually in a lower peak in the buffer region. With also an extension of the log-law region (mainly due to the increase of the boundary layer size due to increasing roughness).

Figure 14 and 16 from Flack [80] presents both skewness and kurtosis for streamwise velocity component in turbulent boundary layer region. The roughness introduces a higher value of skewness in the wall bounding proximity and a lower value of skewness when at the boundary layer limit. The kurtosis shows a higher value of kurtosis at the boundary layer limit when roughness is applied to the surface.

All the before-mentioned studies present features introduced by roughness that are extremely close to what we have experience with increasing surface temperature. With roughness, we do observe the buffer region being less affected by the viscosity contribution in favor of more prominent advection [79, 80]. Figure 4.15 shows the same effect for increasing surface temperature, where the buffer region does result in a more log-like profile when the temperature increases. This idea is also supported by the expected instability induced by the heating from below induced by layer instabilities ([48] p.473). It is anyway important to point that roughness is an impenetrable obstacle with a defined pattern, the same cannot be said for any effect that heat could introduce. We may try to justify this similarity as small thermal plumes raising as soft roughness introducing vertical momentum and interacting with the viscous sub-layer in a similar manner to what can be observed in roughness. If this is true we may expect an even closer similarity to our effect when investigating soft roughness contribution in turbulent boundary layers and especially in the viscous sub-layer (i.e. a study of algae and fungus on a bed river). We 
have unfortunately not found an experimental work that focus on this specific argument and this is a testament to how important is to keep an high focuses on this field.

In the aforementioned roughness studies, the boundary layer size is affected by roughness and the wake transition seems not to be affected by roughness. Nevertheless, we do observe a peculiar increase in velocity in our wake region that leads to a boundary layer size similar in all our measurements, despite the transformations induced by the heat in the viscous sub-layer. One of the elements that can affect more the wake region in a similar way to what we observe is the pressure gradient. Spalart in 1992 [82] published an extremely helpful experimental/numerical work in which we can understand how a favorable pressure gradient (FPG) or adverse pressure gradient (APG) can affect the boundary layer wake.

In sec. 3.1 we present our analysis of the flow velocity inside our wind-tunnel along its entire test section. We do observe a weak increase in velocity along the streamwise direction, this increase of velocity is observed to be below the $2-3 \%$. This increment is somehow expected because our wind-tunnel has a simple design and cannot correct for the weak pressure gradient developed by the growth of the boundary layer along the windtunnel walls, as a plus the introduction of our experiment inside the wind-tunnel section reduces the flow section and therefore an increase in flow velocity is required to keep the mass flow constant. We do observe then that our experiment is affected by a weakly FPG. When our thermal surface exchanges heat with the flow the thermal expansion induced by that is expected to drive to an even stronger FPG effect. One could then expect that the introduction of heat in our boundary layer would result in a turbulent boundary layer closer to what observed in FPG, in particular in the wake region where the effects of the pressure gradients are usually more prominent.

Our wake instead shows an unexpected result. The wake region in our experiments shows an evolution of the wake for increasing $G r_{x}$ that recalls what is usually observed in an APG boundary layer.

For an FPG-boundary layer (see e.g., [82]), the viscous sub-layer becomes bigger (and therefore an increased viscosity effect area), while the wake region becomes smaller, with a smaller difference of the velocity and the log-profile (i.e., the Musker curve) there. In contrast, APG results in a smaller buffer region (and therefore a decreased viscosity effect area) and a larger wake region with a larger overshoot of the velocity compared to the 

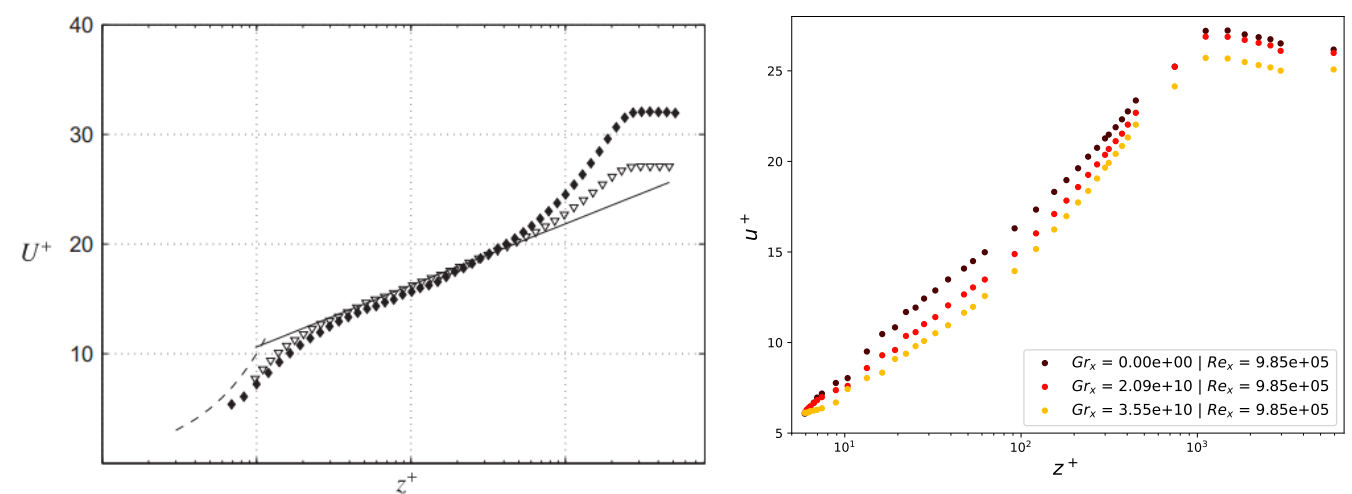

Figure 4.26: Left - Comparison of velocity boundary layer for a flow with $R e_{\tau} \approx 2800$. Empty symbols represent Zero pressure gradient, Full symbols represents Adverse pressure gradient. Plot from Monty 2011[83]. Data extracted from fig.4.15 for reference

log-law (see e.g., $[82,83]$ ). A comparison between an APG and ZPG boundary layer is shown in fig.4.26[83].

Given the complexity of the phenomenons observed in our study, it is not simple to point out a single mechanism that can justify all our observations. Considering that we expect a favourable pressure gradient, the result observed here seems to contradict at a first glance.

In this data-set the shear stress is dominating over the buoyant force and therefore we consider it in a forced convection regime. The dominance of the shear force does not exclude a contribution of the buoyant forces to the evolution of the boundary layer. The buffer region represents a region of transition between the laminar and turbulent flow, with a critical value of shear stress that when crossed drives to the transition from steady to unsteady flow. Viscosity and shear force are competitive effects, the buffer region represents the region of equilibrium between the instability promoted by the shear stress and the viscosity that opposes to the induced instability. The increase of $G r_{x}$ is expected to result in contribution toward flow instability. In steady flows (i.e. RayleighBénard convection) it has been observed that there is a minimum temperature to trigger the generation of thermal plumes for a given geometry and fluid properties and this is a similarly competitive effect to what is expected in the buffer region. It is fair to assume that both shear force and buoyant effects contribute to instability against viscosity. It is also to be noted that the increase of $G r_{x}$ in air (our fluid) increases both kinematic and dynamic viscosity.

If we consider buoyancy effects negligible it is possible to conclude that for increasing 
temperature we expect to observe in the buffer region a shift of the equilibrium toward a more viscous and therefore laminar buffer region. We do observe that the introduction of the heat, despite the increase in viscosity shift the buffer layer toward a less stable regime, where we observe a less pronounced hump and a shift of the log-law velocity to lower altitudes. The increase of the instability observed in the buffer region when $G r_{x}$ increases show a non-negligible buoyant effect.

The effect of heat in the wake region is a bit harder to explain. We have discussed the similarities between the APG boundary layer and the effect of the heat over the wake region in our experiment. This result does not fit with the expected FPG in our experimental environment. During the initial design, we did not expect an eventual pressure gradient to be relevant in our measurements and therefore no measurement system has been considered to monitor this value along $\mathrm{x}$. We feel that the data available to us does not allow us to offer a solid explanation for the effect of heat on the wake region in our experiment. More experiments with an eye on the pressure gradient developed when temperature increases are of utmost importance to propose a solid hypothesis on this phenomenon.

\subsubsection{Velocity boundary layer in the transitional regime with heated surface}

In the previous section, we have investigated how heating from below can alter the streamwise velocity field in a fully turbulent boundary layer where shear forces dominate over buoyancy. While a quantitative change was observed, we do expect to see a stronger effect when the shear flow is weaker and therefore buoyancy plays a more important role.

By using the camera setup described in sec. 3.6 we have been able to record videos of the flow under weak shear forces. These videos show nicely the interaction of the shear forces and the thermal plumes that are generated due to buoyancy. In fig. 4.27, 4.28, and 4.29 we present snapshots of the smoke-seeded flow at various times for the smallest inflow velocity, i.e., $U_{0}=0.3 \mathrm{~m} / \mathrm{s}$ and $R e_{x}=4.7 \times 10^{4}$ and different $\mathrm{Gr}_{x}$.

Videos available online [84-88]. Figure 4.27 is collected with $R e_{x}=4.7 \times 10^{4}$ and $G r_{x}=0$. We can understand a bit more about the flow and its properties by looking at the evolution of the smoke structure. In a laminar flow, we do expect any exchange of 


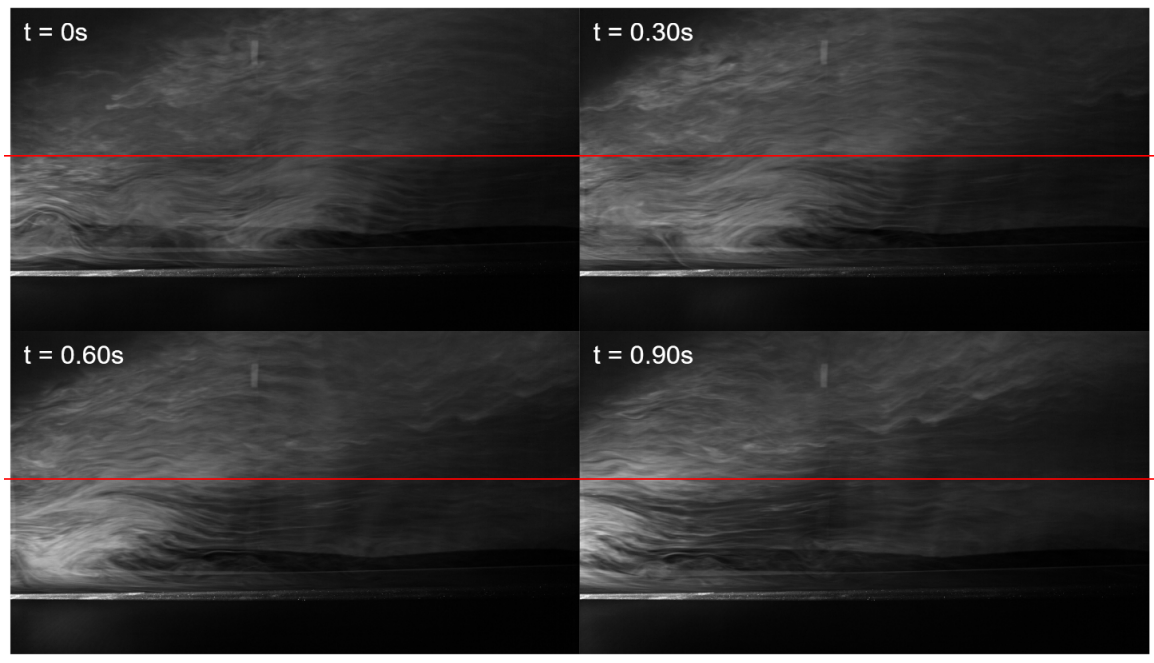

Figure 4.27: Snapshot of the smoke-seeded flow field with $R e_{x}=4.7 \times 10^{4}$ and $G r_{x}=0$. Red lines mark the approximate boundary layer height $(\delta=7.5 \mathrm{~cm})$, estimated from velocity measurements at $G r_{x}=0$, Movie available online [84].

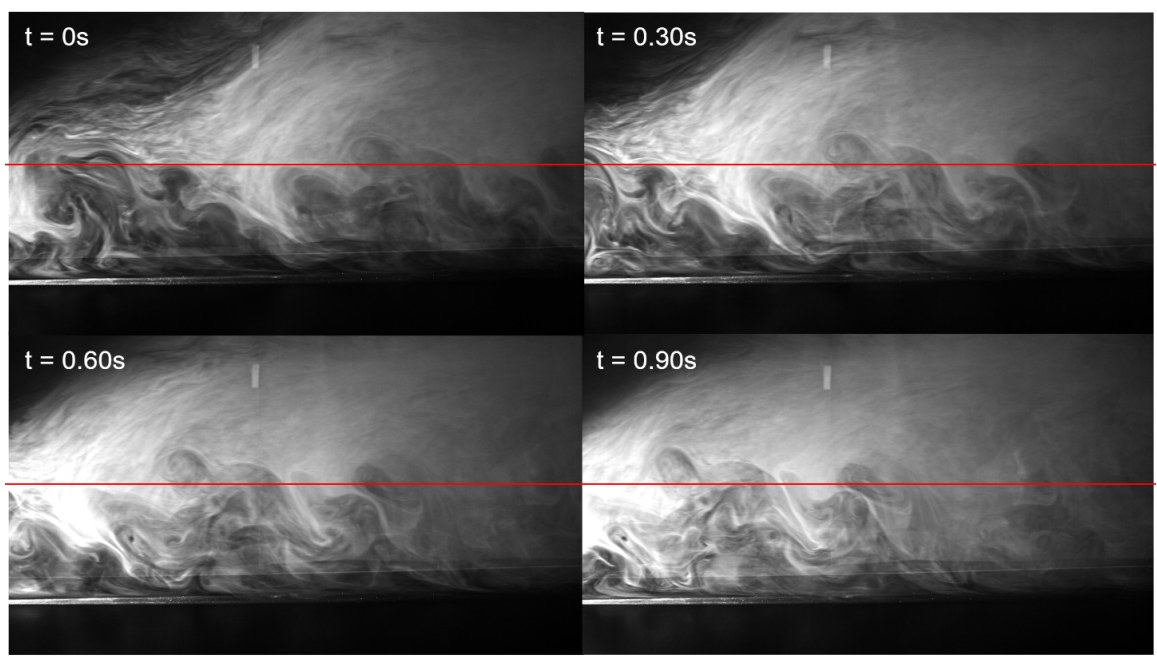

Figure 4.28: Similar to fig. 4.27 but for $G r_{x}=1.04 \times 10^{10}$, Movie available online [85].

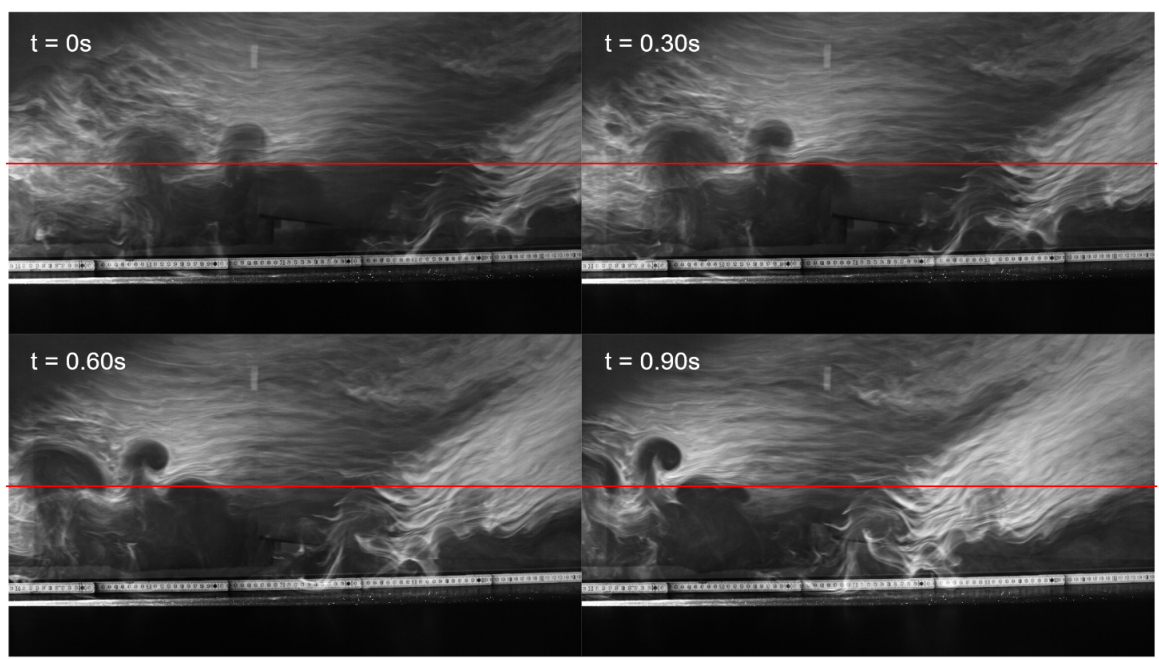

Figure 4.29: Similar to fig. 4.27 but for $G r_{x}=6.26 \times 10^{10}$, Movie available online [86]. 
mass between the different layers, and therefore every structure of the flow should stay constant in space and time. And indeed, this is what we see. There are no mixing effects, the general shape the same over the shown time duration, but the lower layers move slower than the top layer as is expected from a structure inside a boundary layer flow.

In fig. 4.28 we can observe a flow with moderate heating applied, with $G r_{x}=1.04 \times$ $10^{10}$. In this figure, we already observe significant differences in the structure compared to the flow at $G r_{x}=0$ fig. 4.27. Mushroom-shaped plumes can be seen rising from the warm surface and crossing the entire boundary layer up to the bulk. The particles used for flow visualisation are droplets produced by vaporisation of a glycerol solution and subsequent condensation of the gas into tiny droplets. As result, the droplets are warmer than the incoming flow $T_{0}$, heat up their close vicinity and rise due to buoyancy towards the upper part of the wind-tunnel. Therefore, seeding densities are usually higher in the upper part of the incoming (laminar) flow than in the lower part. Very close to the plate, the seeding density is smaller than in the bulk flow. This is mainly for two reasons. First, particles that are carried by the bulk do not easily enter the lowest parts of the boundary layer because the z-component of the velocity is small there. This affects particularly the small droplets that follow the flow and are not significantly influenced by gravity. Secondly, particles that get too close to the wall might get in contact with it and adhere to it. In this way, the plate acts as a sink for droplets. This is particularly the case for larger droplet that settle down due to gravity. This plays in our favor since it allows us to easily identify plumes that are ejected from layers closest to the wall.

In fig. 4.29 we apply a strong heating resulting in $G r_{x}=6.26 \times 10^{10}$. The buoyancy here is strong and hot plumes are generated along the entire plate surface and length. In this time sequence, the thermal structures are well defined and can easily be followed in time as they both rise through buoyancy and also get carried in the streamwise direction by the mean flow. By comparing the seeding density (or structures brightness) of fig. 4.28 and fig. 4.29 we can already tell that the mixing between the thermal plumes and the rest of the boundary layer is stronger in the weakly heated experiment. The darker structures indicate that the majority of the air contained in these structures originated from the regions very close to the surface, i.e., from the viscous sub-layer and buffer region. We can also observe that when the surface is warmer the plumes reach significantly larger distances $z$ which are clearly above the velocity boundary that develops without heating 
for $\mathrm{Gr}_{x}=0$ as indicated by red horizontal lines in fig. 4.27, 4.28, and 4.29.

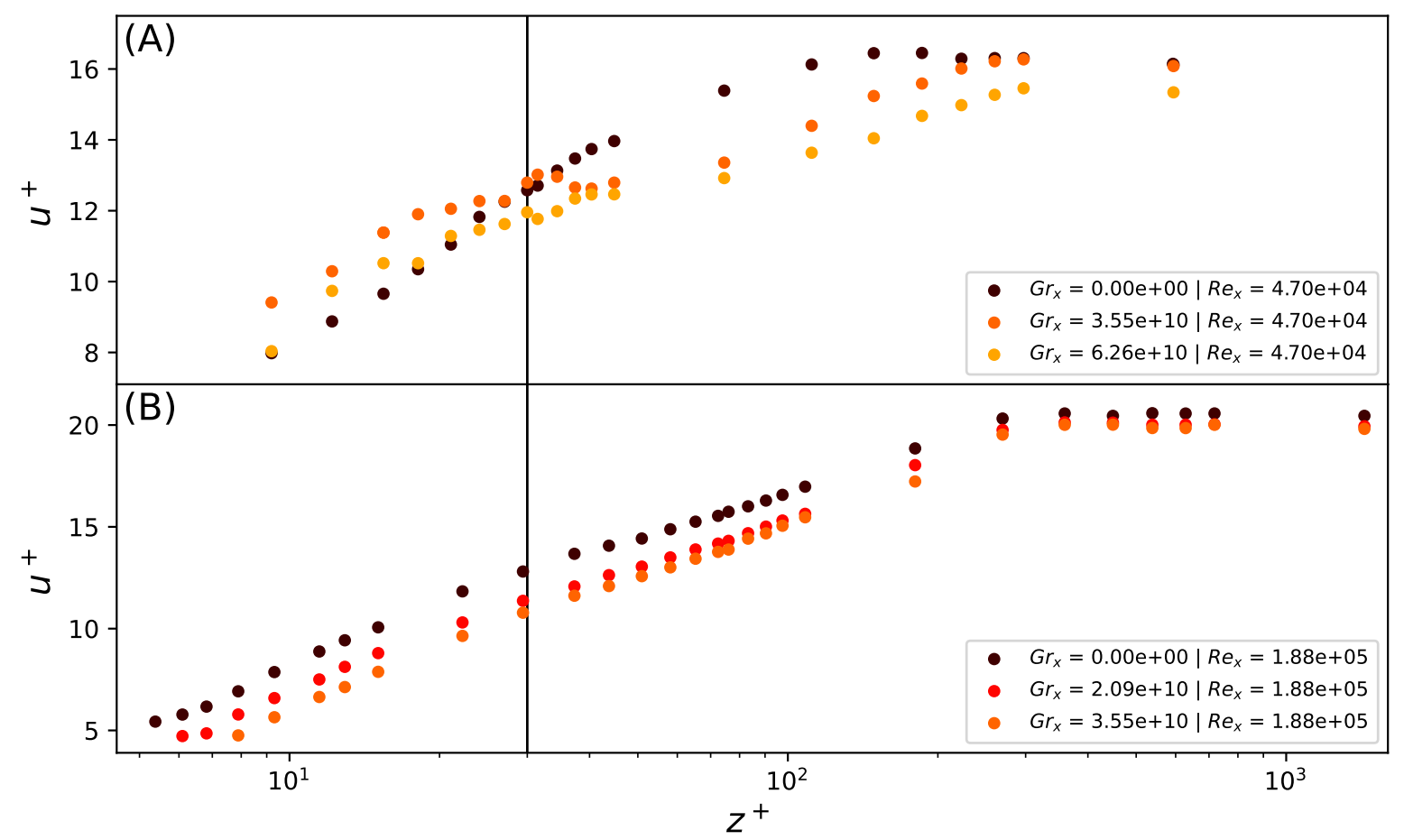

Figure 4.30: Quasi-turbulent velocity boundary layer comparison for different surfaces temperatures. Shown are time average streamwise velocities as function of the distance to the wall $z^{+}$.

After we have gained a qualitative understanding of the heated transitional boundary layer, we again want to look at quantitative changes of the velocity when heat is applied. The average streamwise velocity is shown in fig. 4.30 for different wall temperatures. When the shear stress is low $\left(R e_{x}=4.7 \times 10^{4}\right.$, fig. $4.30(\mathrm{~A})$ and the surface temperature is equal to the flow temperature $\left(G r_{x}=0\right)$, the boundary layer is in a transitional regime (from laminar to turbulent) as already discussed in sec. 4.1.2. Because the boundary layer is not fully turbulent, the $z^{+}$-range, where viscosity does not play an important role for the momentum transport, is very small and hence a clear log-region does not develop. When $G r_{x}$ increases we observe an increase in the average velocity for $z^{+}<30$ and a decreased average velocity above. The velocity profile for $G r_{x}>0$ may suggest the growth of a log-like profile due to the introduction of additional turbulent kinetic energy. Furthermore, also the boundary layer size $\delta$ increases for $\operatorname{Gr}_{x}>0$, which also agrees with the assumption that additional turbulent kinetic energy is introduced via rising thermal plumes which transform potential energy into kinetic energy and in this way also enhance 
the transport of momentum in wall-normal direction. The boundary layer for the two $\mathrm{Gr}_{x}>0$ is roughly twice as large as for $\mathrm{Gr}_{x}=0$. Note that the difference between $\delta$ for the two $\mathrm{Gr}_{x}>0$ is below our spatial resolution.

We see in fig. 4.30(B) that already for slightly larger $\operatorname{Re}_{x}=1.88 \times 10^{5}$ the influence of an increased $\mathrm{Gr}_{x}$ on the average velocity is qualitatively different. Now, as shown above, the boundary layer is much closer to a fully turbulent state. Also, similar to a turbulent boundary layer, a finite $\mathrm{Gr}_{x}$ reduces $u^{+}$throughout the entire boundary layer. This observation hints that the boundary layer height also increases with increasing $\mathrm{Gr}_{x}$. However, again also here, our spatial resolution is too small to resolve the boundary layer height sufficiently well. Also, the wake is clearly defined when $\mathrm{Gr}_{x}$ increases.

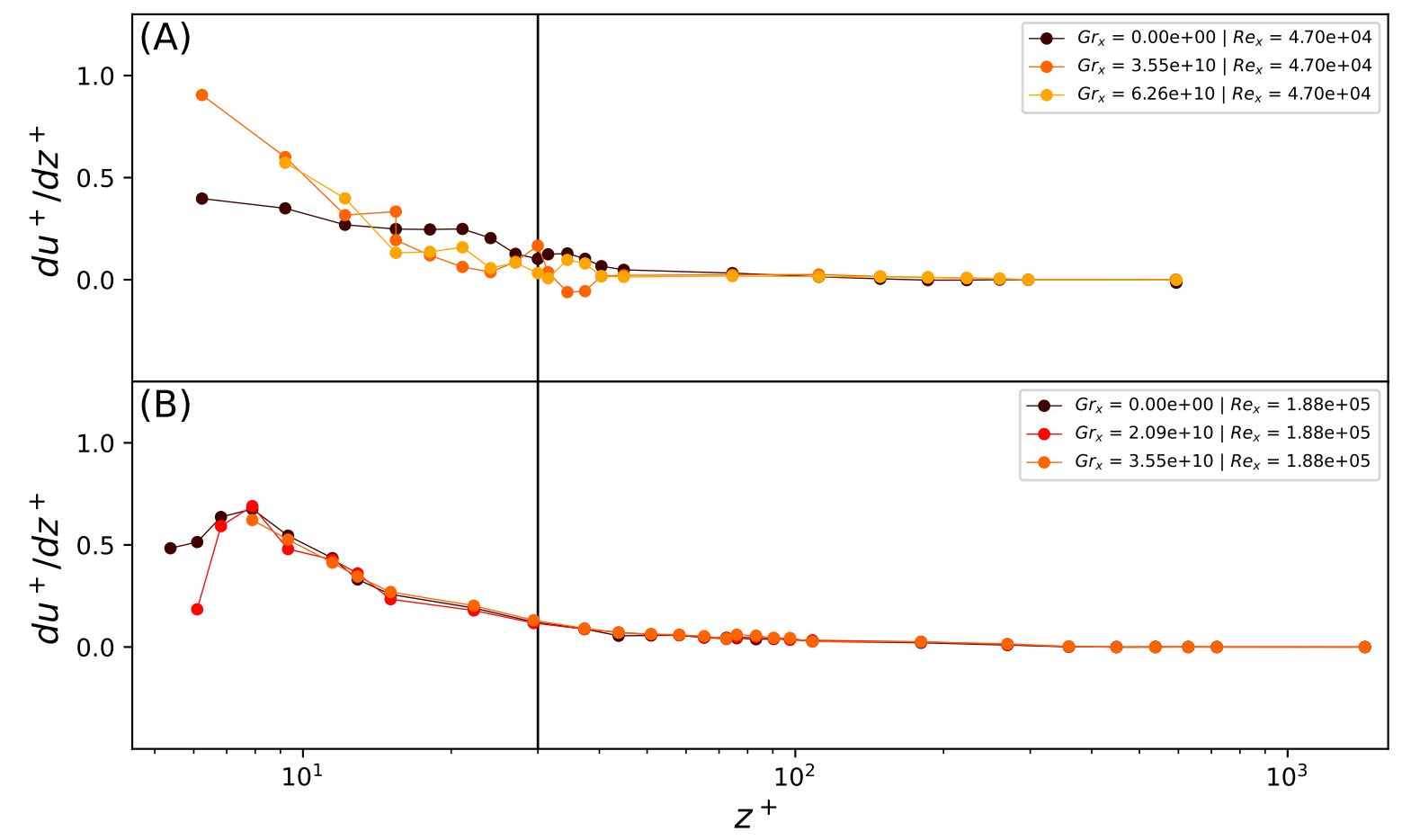

Figure 4.31: Velocity gradient in the boundary layer with different surface temperatures, for small $R e_{x}$.

Figure 4.31 presents the velocity gradient inside quasi-turbulent boundary layers. This analysis teaches us something about the local momentum transfer. However, one should not forget that momentum is also advected with the mean flow in a downstream direction. When shear stress is weak (at $R e_{x}=4.7 \times 10^{4}$ ) the velocity gradient in the surface proximity increases with increasing $\mathrm{Gr}_{x}$ for $z^{+}<15$ or so.

In fig. 4.32 we show the normalized standard deviation of our data-sets for the two 


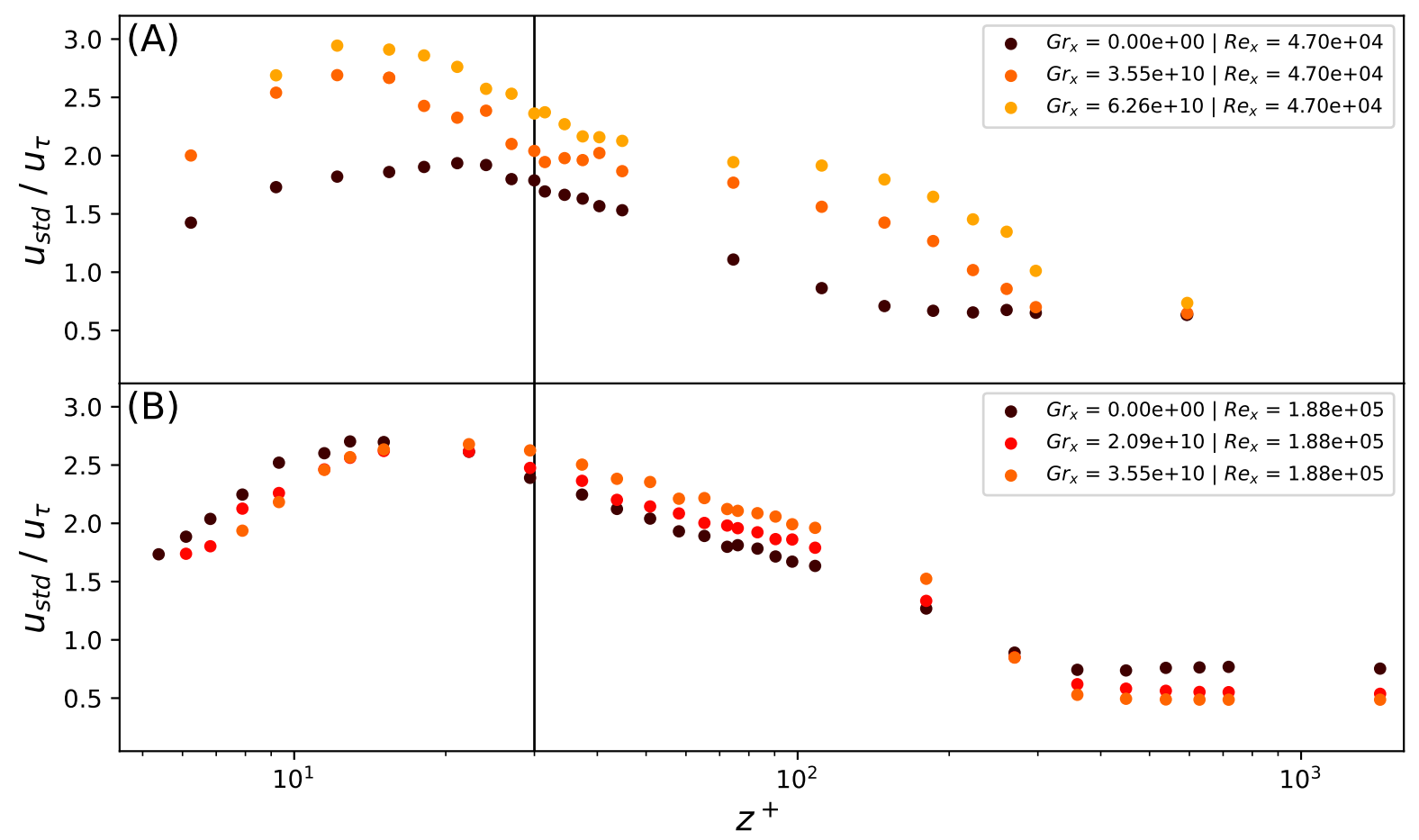

Figure 4.32: Quasi turbulent boundary layer standard deviation for different temperatures

smallest $\operatorname{Re}_{x}$. The influence of heating from below is particularly strong for $\operatorname{Re}_{x}=4.7 \times 10^{4}$ (fig. 4.32A). There, the maximum of $u_{s t d}$ increases and shifts to smaller $z^{+}$, closer to the wall. In fact, for finite $\mathrm{Gr}_{x}$, the maximum is at $z^{+} \approx 15$ or so, at the same $z^{+}$, where a maximum also occurs for the fully turbulent boundary layer. Also for $z^{+} \approx 100$ another hump appears, resulting in an $u_{\text {std }}\left(z^{+}\right)$profile that appear surprisingly similar to the fully turbulent boundary layer.

Also for faster bulk flow velocities $\left(\operatorname{Re}_{x}=1.88 \times 10^{5}\right)$, do we observe strong differences where the increase in the shear stress pushes the flow closer to the turbulent transition. There, only outer regions (the wake) are clearly affected by the increase of $\mathrm{Gr}_{x}$. There, $u_{s t d}$ growth with increasing $\mathrm{Gr}_{x}$, while the maximum close to the wall (at around $z^{+} \approx 15$ ), is barely affected. Even more, at $z^{+} \approx 10$ the fluctuations seem to decrease with increasing $\mathrm{Gr}_{x}$, similar to what was observed for the fully turbulent case above.

\section{Power Spectrum}

In fig. 4.33 we compare the probability density function $P\left(u^{\prime}\right)$ at different altitudes for different $\mathrm{Gr}_{x}$. This allows us to better understand how the introduction of heat does 


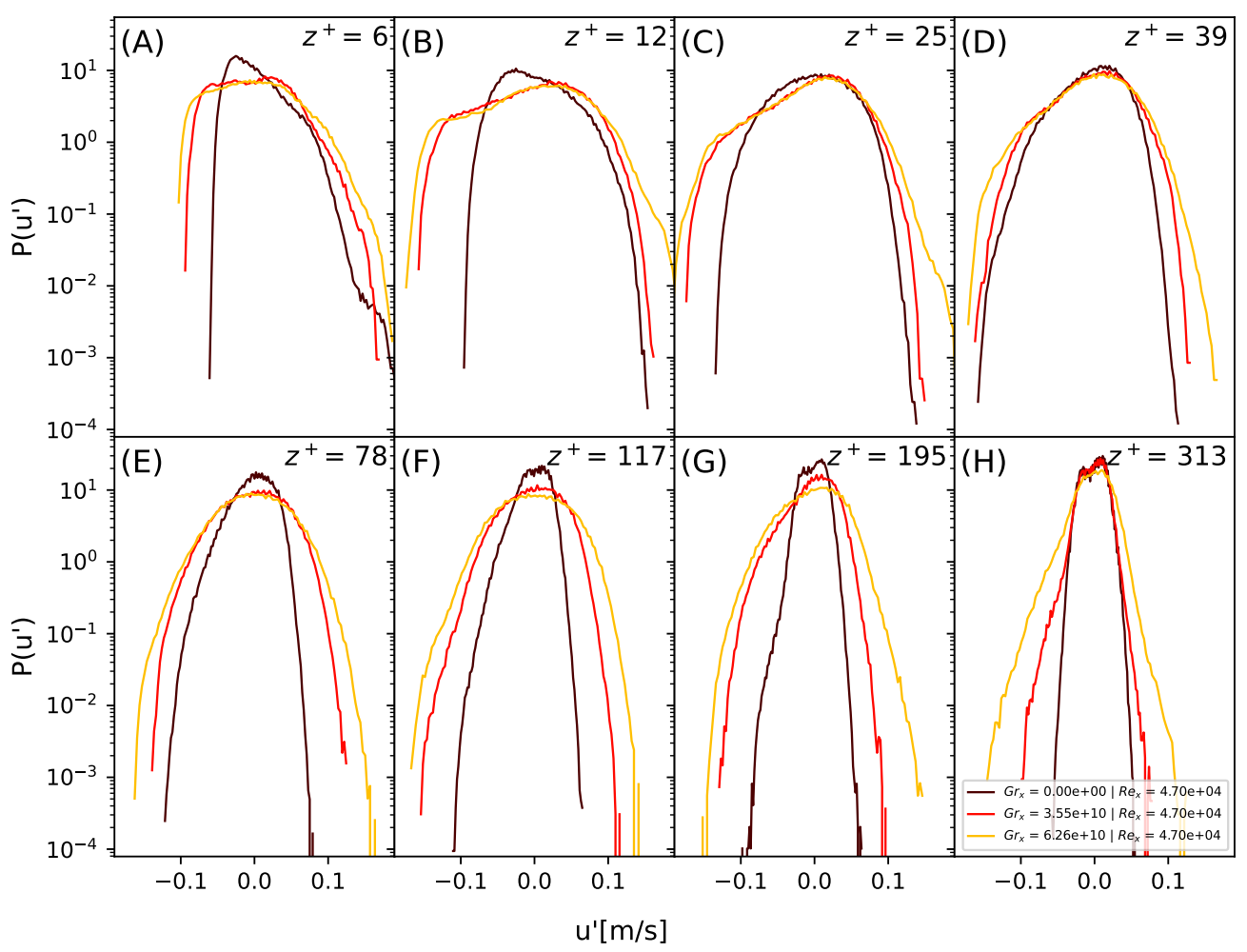

Figure 4.33: Probability density function of velocity fluctuations for different surface temperature and flow velocity $U_{0}=0.3 \mathrm{~m} / \mathrm{s}$ and $R e_{x}=4.7 \times 10^{4}$

affect the flow at different altitudes.

For $z^{+} \leq 25$ fig. $4.33(\mathrm{~A})$, (B), and (C) we do see a clear influence of the heat for the location of the maximum as well as the skewness. We observe the growth of a secondary peak that could suggest a second mode in the velocity fluctuations introduced by the heat, the second peak is centred at $u^{\prime} \approx 0.025 \mathrm{~m} / \mathrm{s}$.

At $z^{+}=39$ fig. 4.33 (D) the heat has the only effect of widening the distribution tail but does not affect consistently the shape close to the maximum, and $P\left(u^{\prime}\right)$ exhibits only a single mode centered around $u^{\prime} \approx 0.025 \mathrm{~m} / \mathrm{s}$.

For $z^{+} \geq 78$ fig. $4.33(\mathrm{E}),(\mathrm{F}),(\mathrm{G})$, and $(\mathrm{H})$ we observe the effect of the heat, far from the surface, are still consistent. One would expect, that with increasing distance from the surface the influence of the warm wall should decrease, but we still can see a wider distribution of velocities, also in the outer regions that are consistently wider for higher surface temperature. The increase of the surface temperature does also increase the boundary layer size for $U_{0}=0.3 \mathrm{~m} / \mathrm{s}$, when the boundary layer is in the transitional regime. Therefore, in the $P\left(u^{\prime}\right)$ analysis from $z^{+}>117$ the $\Delta T 0 K$ and $G r=0$ measurements are in the bulk laminar flow regime, whether the heated surface is still inside the 
boundary layer.

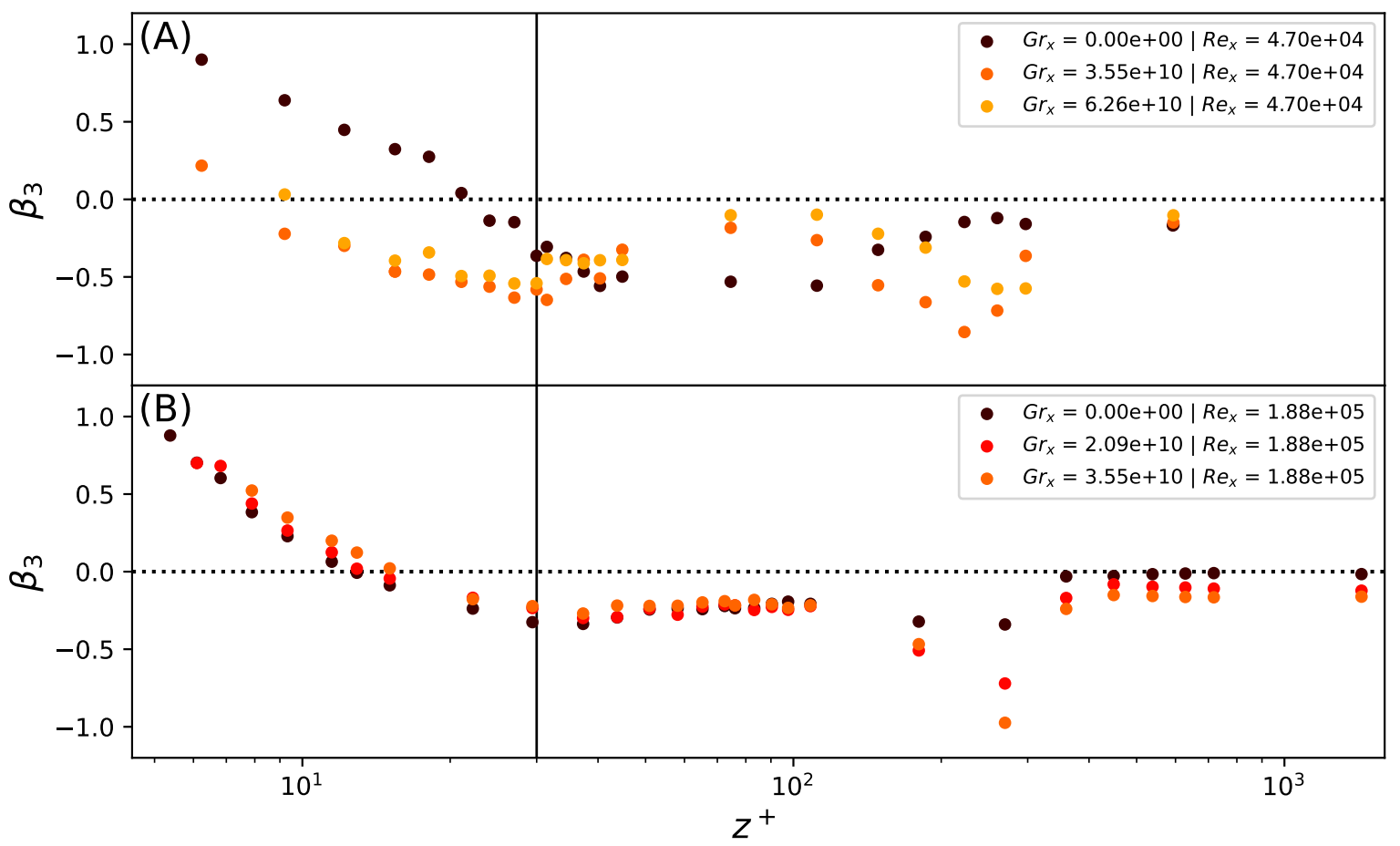

Figure 4.34: Skewness in quasi turbulent boundary layer at different surface temperatures

A similar observation made in fig. 4.32 can also be made when looking at the skewness $\beta_{3}$ (fig. 4.34) and kurtosis $\beta_{4}$ (fig. 4.35), namely that with increasing $\mathrm{Gr}_{x}$, the transitional boundary layer (at $\operatorname{Re}_{x}=4.7 \times 10^{4}$ ) gains characteristics that are more similar to a turbulent boundary layer. For this case $\left(\operatorname{Re}_{x}=4.7 \times 10^{4}\right)$, an increase of the surface temperature results in a reduced skewness in the proximity of the surface for $z^{+}<30$. In fact, $\beta_{3}$ is close to 0 at surface proximity but turns negative further away. The negative skewness so close to the surface is evidence that fluctuations are produced by buoyancy and by shear instabilities. In the region $z^{+} \approx 100$, we see an increased $\beta_{3}$ with increasing $\mathrm{Gr}_{x}$. In the wake region again, an increased $\mathrm{Gr}_{x}$ results in a decreased skewness, similar to what has been already observed in fully turbulent boundary layers. The kurtosis (fig. 4.35(A)) the kurtosis becomes clearly negative in proximity of the surface when $G r_{x}$ increases, induced by the buoyant events. $\beta_{4}$ approaches the 0 already at $z^{+}=15$ rather than $z^{+}=45$ when $G r_{x}>0$. When $z^{+}=190$ and $G r_{x}>0$ we observe the raise in $\beta_{4}$ similar to what has been observed in all our turbulent boundary layers, but not present when $G r_{x}=0$.

For $\operatorname{Re}_{x}=1.88 \times 10^{5}$ the skewness behaves similarly to what has been presented 


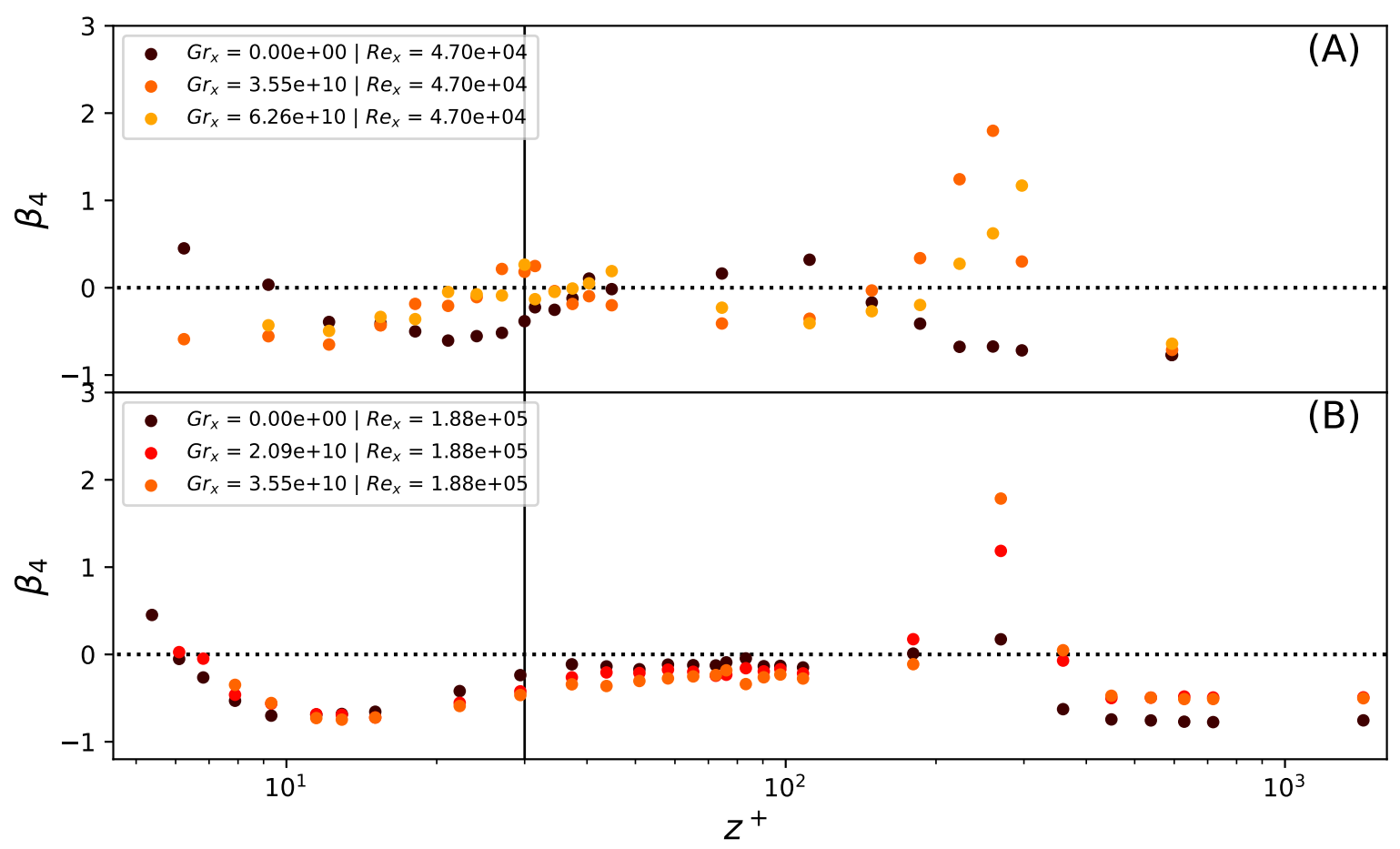

Figure 4.35: Kurtosis in quasi turbulent boundary layer at different surface temperatures

and discussed in fully turbulent boundary layers, with an enhanced asymmetry (negative skewness) in the wake region with increasing $\mathrm{Gr}_{x}$. This results in an enhanced minimum that, on the $z^{+}$scale, similar to the one seen for the turbulent boundary layer.

Similar considerations hold true for the kurtosis $\beta_{4}$ (fig. 4.35) that we have shown is mainly affected by heat only in the wake region for fully turbulent boundary layers. The value of $\beta_{4}$ is affected by the increased heat for $z^{+} \approx 30$ resulting in more "extreme" events in that specific region.

The power spectrum for $R e_{x}=4.7 \times 10^{4}$ fig. 4.36 shows an increase in the turbulent kinetic energy all along the entire boundary layer resulting in a shift of the dissipation range to larger wavenumbers $k$ or smaller length scales. Energy is hence dissipated in smaller eddies. We recall from chapter 2 the size of these eddies scales with the Kolmogorov length $\eta=\left(\nu^{3} / \epsilon\right)^{1 / 4}$. Because the kinematic viscosity $\nu$ increases with temperature, the increase of the dissipation length must be due to an increased energy dissipation rate $\epsilon$, which itself results from increased production of turbulent kinetic energy. The source for this additional energy can only be potential energy due to heating and the resulting buoyancy. This effect was not seen for larger $\mathrm{Re}_{x}$, because the production of turbulent kinetic energy due to buoyancy was negligible compared to the production due to shear stress. 


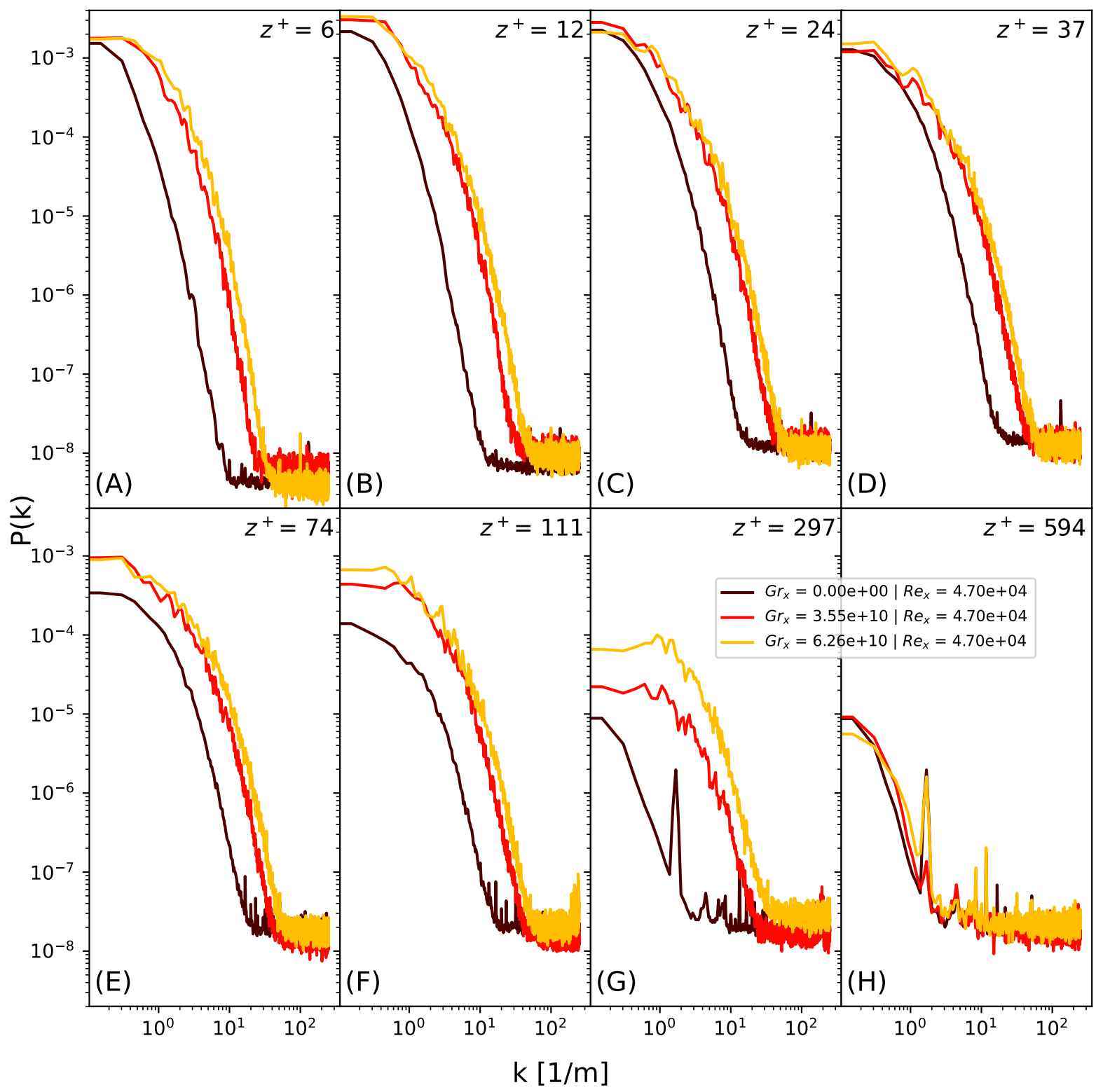

Figure 4.36: Power spectral density for different surface temperature and flow velocity $R e_{x}=$ $4.7 \times 10^{4}$ 
We note that the power spectrum for $R e_{x}=1.88 \times 10^{5}$ shows a qualitatively similar but weaker effect, therefore it is not presented here for simplicity.

The destabilising effect of heat and the additional production of turbulence due to buoyancy is also neatly visible in fig. 4.37, where the turbulent intermittence $\gamma$ is shown as function of $z^{+}$.

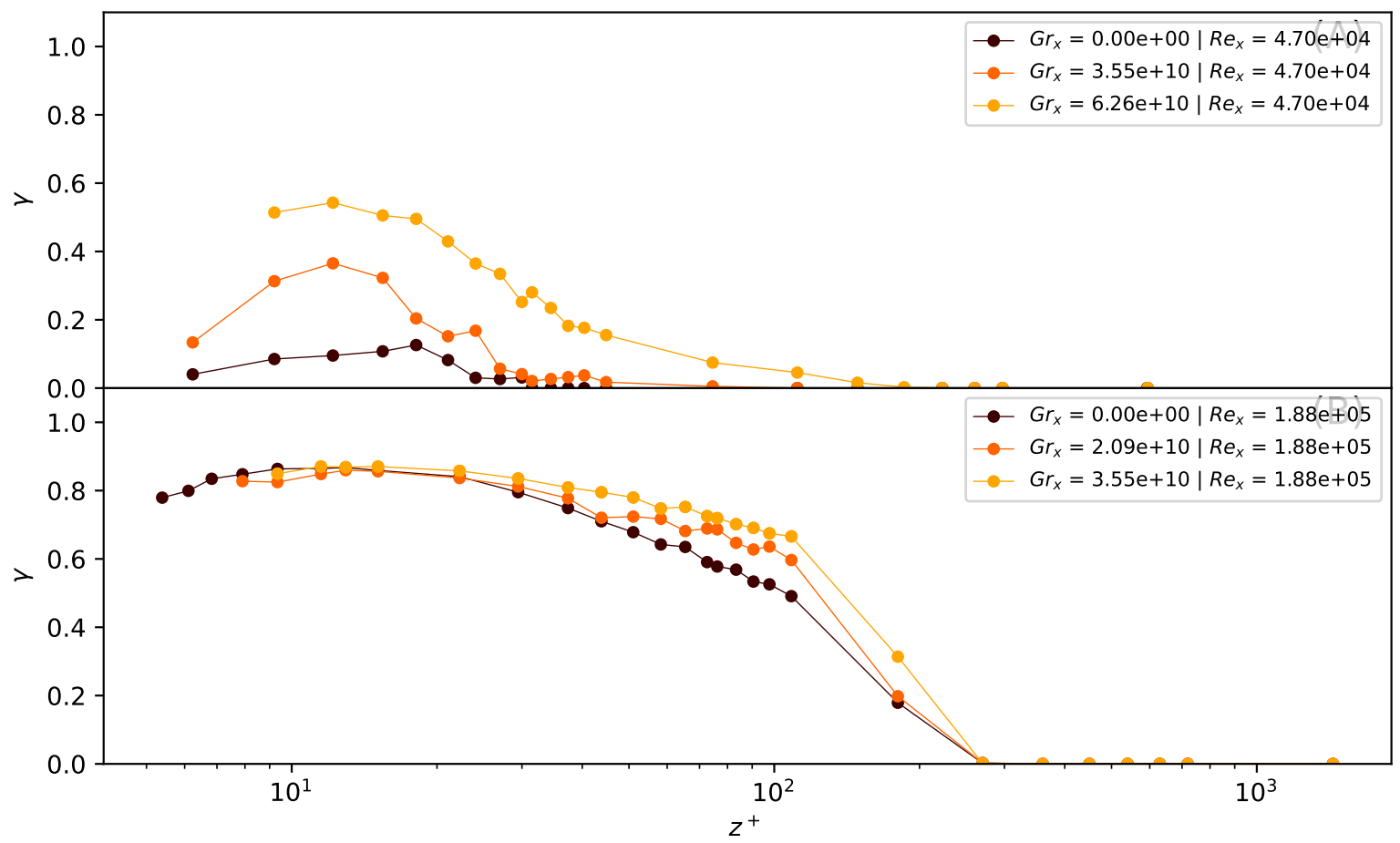

Figure 4.37: Turbulence intermittency for different surface temperature, with flow velocities $U_{0}=0.3 \mathrm{~m} / \mathrm{s}$ and $U_{0}=1.2 \mathrm{~m} / \mathrm{s}$.

The introduction of heat does in the weakly sheared flow $R e_{x}=4.7 \times 10^{4}$ increase the turbulence intermittency inside the boundary layer, clearly moving the boundary layer toward a more turbulent regime. This allows us to confirm that the introduction of heating from the bottom in a weakly sheared flow triggers an earlier transition toward more turbulent regimes. Despite the before mentioned argument, it is also necessary to note that this effect becomes less relevant with increasing $\operatorname{Re}_{x}$. Already at the slightly larger $R e_{x}=1.88 \times 10^{5}$ is the turbulent intermittency significantly less affected by the increase of $\mathrm{Gr}_{x}$, with a clear influence mostly in the $\log$ /wake.

The analysis of the $\gamma$ also confirms that a heated surface does induce the boundary layer to an earlier transition from laminar to turbulent, but also that this effect is clearly more prominent for weak shear stresses. When the shear flow increases, the contribution 


\begin{tabular}{||ccc||}
\hline$R e_{x}$ & $G r_{x}$ & $\langle\gamma\rangle$ \\
\hline \hline $4.70 \times 10^{4}$ & 0 & 0.037 \\
\hline $4.70 \times 10^{4}$ & $3.55 \times 10^{10}$ & 0.095 \\
\hline $4.70 \times 10^{4}$ & $6.26 \times 10^{10}$ & 0.242 \\
\hline $1.88 \times 10^{5}$ & 0 & 0.662 \\
\hline $1.88 \times 10^{5}$ & $2.09 \times 10^{10}$ & 0.679 \\
\hline $1.88 \times 10^{5}$ & $3.55 \times 10^{10}$ & 0.743 \\
\hline
\end{tabular}

Table 4.5: Experimentally determined turbulent intermittency obtained for different surfaces temperatures. $\langle\gamma\rangle$ represents the average of the gamma for $z<\delta_{99}$

of heat become less effective. This result suggests that the increased thermal energy does destabilize the transitional boundary layer region, forcing the flow to an earlier transition to fully turbulent, resulting then in a lower $\mathrm{Re}_{\text {crit }}$.

\subsubsection{Shear velocity correction and modeling}

In the previous section, we have investigated the changes introduced by the surface heat on velocity statistics inside the boundary layer. For this, we have expressed both the velocity, as well as the wall-normal coordinate in wall units. Since we wanted to directly compare the measurements with results obtained with $\mathrm{Gr}_{x}=0$, we have normalised all measurements by the same shear velocity $u_{\tau}$, which was determined for $\mathrm{Gr}_{x}=0$ also for larger $\mathrm{Gr}_{x}$. We know from our previous analysis that heating from below causes qualitative changes of the velocity field and hence the average streamwise velocity might not be well represented by the Musker curve and we cannot collapse data for different $\mathrm{Gr}_{x}$ by simply varying $u_{\tau}$. In this section, we want to attempt a different fitting method with a new set of fitting parameters and present our findings.

Rodriguez-Lopez in 2015 [89] introduced a method that allows a more complex fit of the turbulent boundary layer. These methods promise to be able to fit the fully turbulent boundary layer even for small $R e_{x}$ with shear velocity $u_{\tau}$, boundary layer thickness $\delta$, wake parameter $\Pi$, the von Karman Constant $\kappa$, and correct the potential error on the $z_{0}$ height with a shift that we named $\epsilon$. 
The entire method is listed in [89]:

$$
\begin{aligned}
z^{*} & =z-\epsilon \\
u_{\text {canonical }} & = \begin{cases}u_{\text {musker }}^{+}+\frac{2 \Pi}{\kappa} W\left(z^{*} / \delta\right), & 0 \leq z^{*} \leq \delta \\
U_{0}^{+}, & \delta \leq z^{*} \leq \infty\end{cases}
\end{aligned}
$$

with $u_{\text {musker }}^{+}$being the Musker curve, $U_{0}^{+}=U_{0} / u_{\tau}$ the normalised bulk velocity, $\kappa=0.41$ the von Karman constant, and $W$ being the wake function defined as:

$$
W(\eta)=\frac{1-\exp \left[-(1 / 4)\left(5 a_{2}+6 a_{3}+7 a_{4}\right) \eta^{4}+a_{2} \eta^{5}+a_{3} \eta^{6}+a_{4} \eta^{7}\right]}{1-\exp \left[-(1 / 4)\left(a_{2}+2 a_{3}+3 a_{4}\right)\right]} \times\left(1-\frac{1}{2 \Pi} \ln (\eta)\right)
$$

where $\eta=z^{*} / \delta, a_{2}=132.8410, a_{3}=-166.2041$, and $a_{4}=71.9114$. Note, we do not apply the correction $u_{\text {bump }}^{+}$as suggested in [89]. The final value of shear velocity $u_{\tau}$ is extremely close to what we observed with the classical Musker fit approach when no heating is applied to the surface.

We now fit eq. 4.8 to the average velocities measured at different $\mathrm{Gr}_{x}$, so to gain $u_{\tau}$ as one of the fit parameters. The other fit parameters are the boundary layer height $\delta$, the wake parameter $\Pi$ and a shift $\epsilon$. The re-calculated velocities using the new $u_{\tau}$ are shown in fig. 4.38. Plotted in this way, data for different $\mathrm{Gr}_{x}$ now neatly collapse for the inner part of the boundary layer and diverge in the wake. We also note that the data follow nicely eq. 4.8 and hence we also get information of the velocity in the upper end of the boundary layer, where the data are sparse. We obtained promising results with the fitting parameters obtained listed in table 4.6.

While we have applied this method to all data sets, it is expected to only work well for a sufficiently turbulent boundary layer, i.e., $R e_{x} \geq 1.88 \times 10^{5}$. If by applying this method it is possible to obtain a satisfying collapse, we can assume that the influence of a change in $\mathrm{Gr}_{x}$ on the boundary layer can be represented by only these parameters. From the table 4.6 we can observe that our parameters change consistently when the heat increases and that may lead to validate this approach.

In fig 4.39 we present the difference between the best fits of eq. 4.8 and the real velocities. We can observe that the increase of surface temperature results usually in a general increase of the difference along the entire boundary layer. 


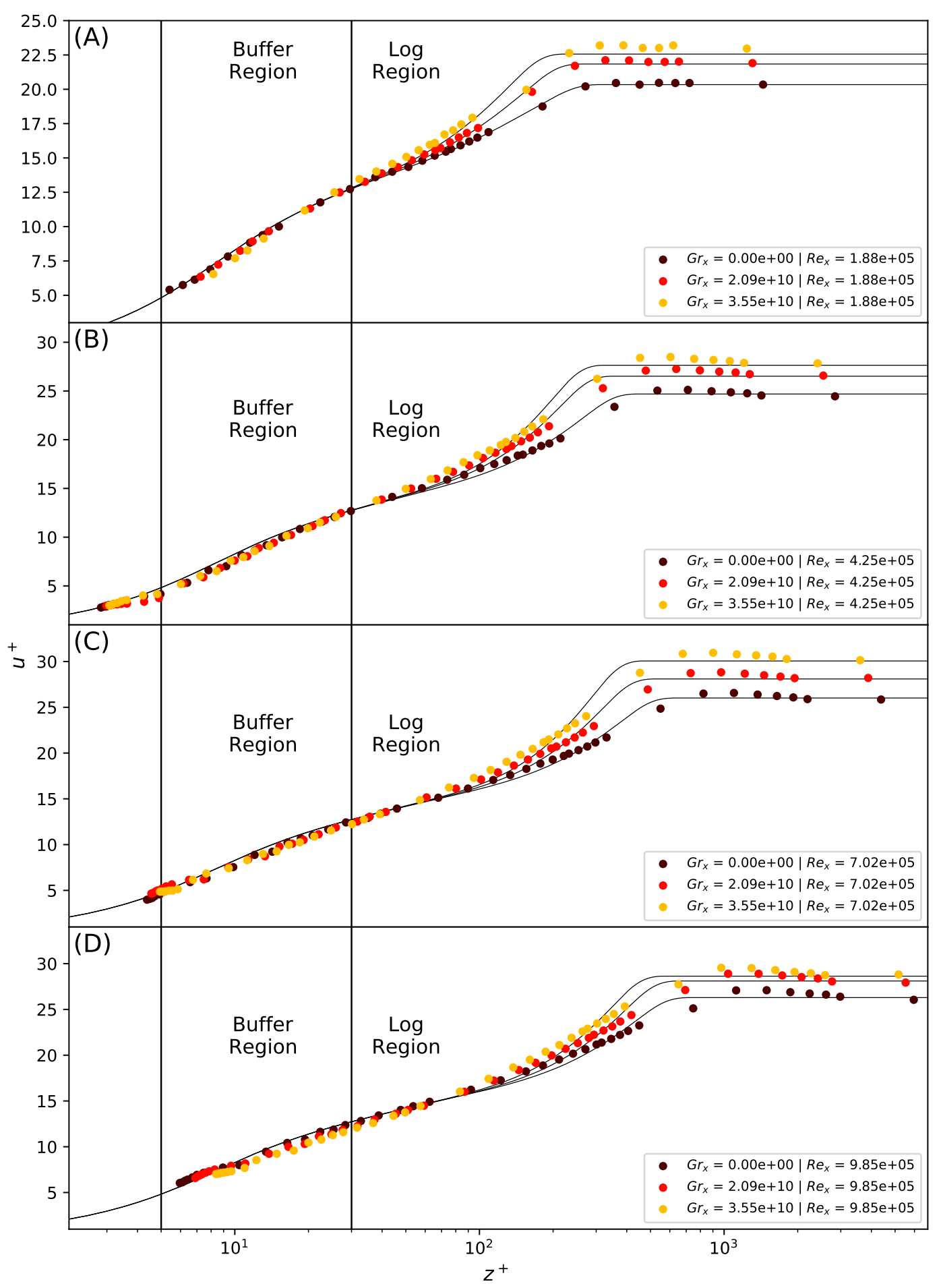

Figure 4.38: Velocity profile at different velocities and temperatures. Fitting parameters are listed in table 4.6. 


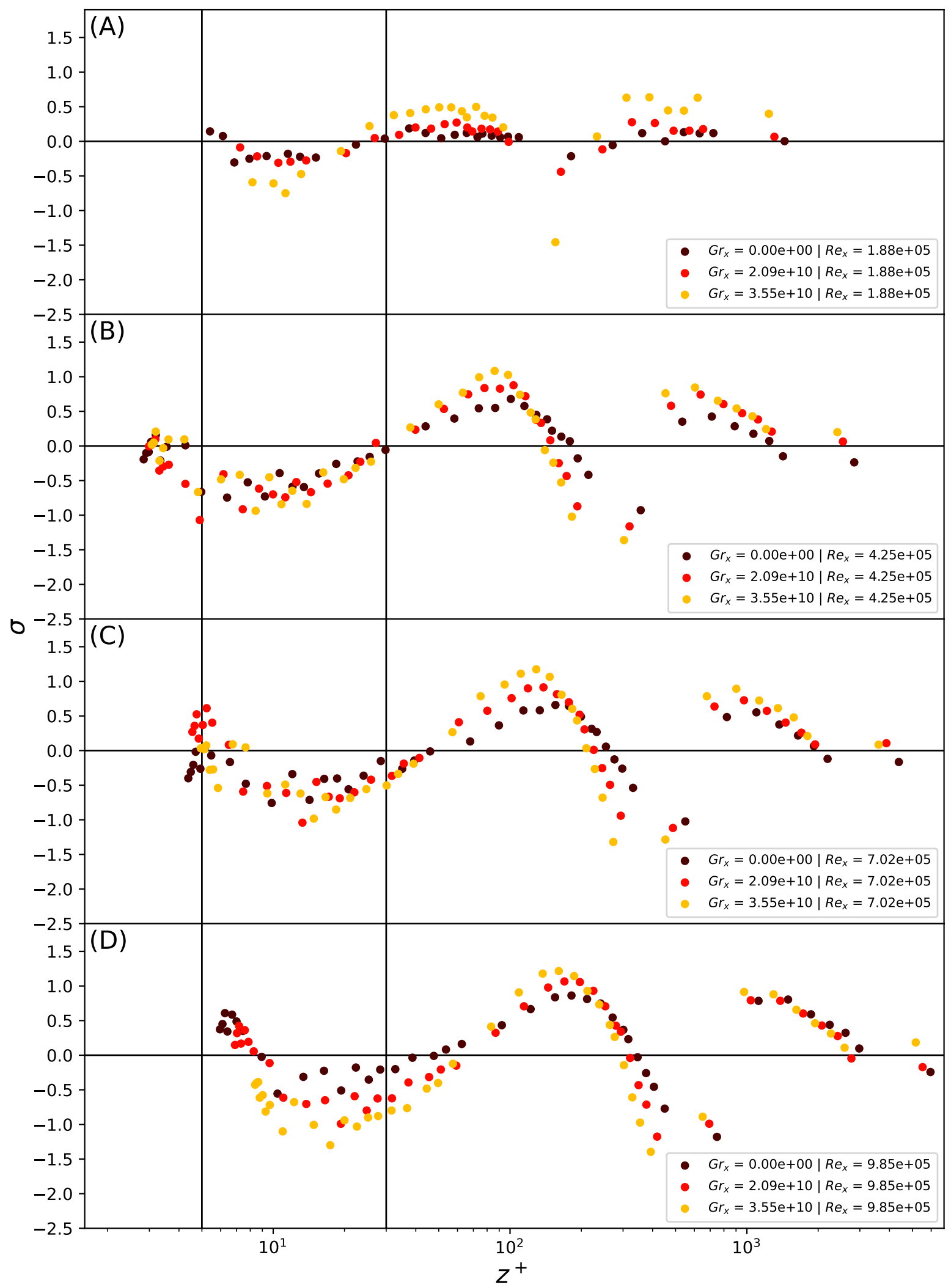

Figure 4.39: Velocity deviation from the fit line obtained with table 4.6 parameters. 


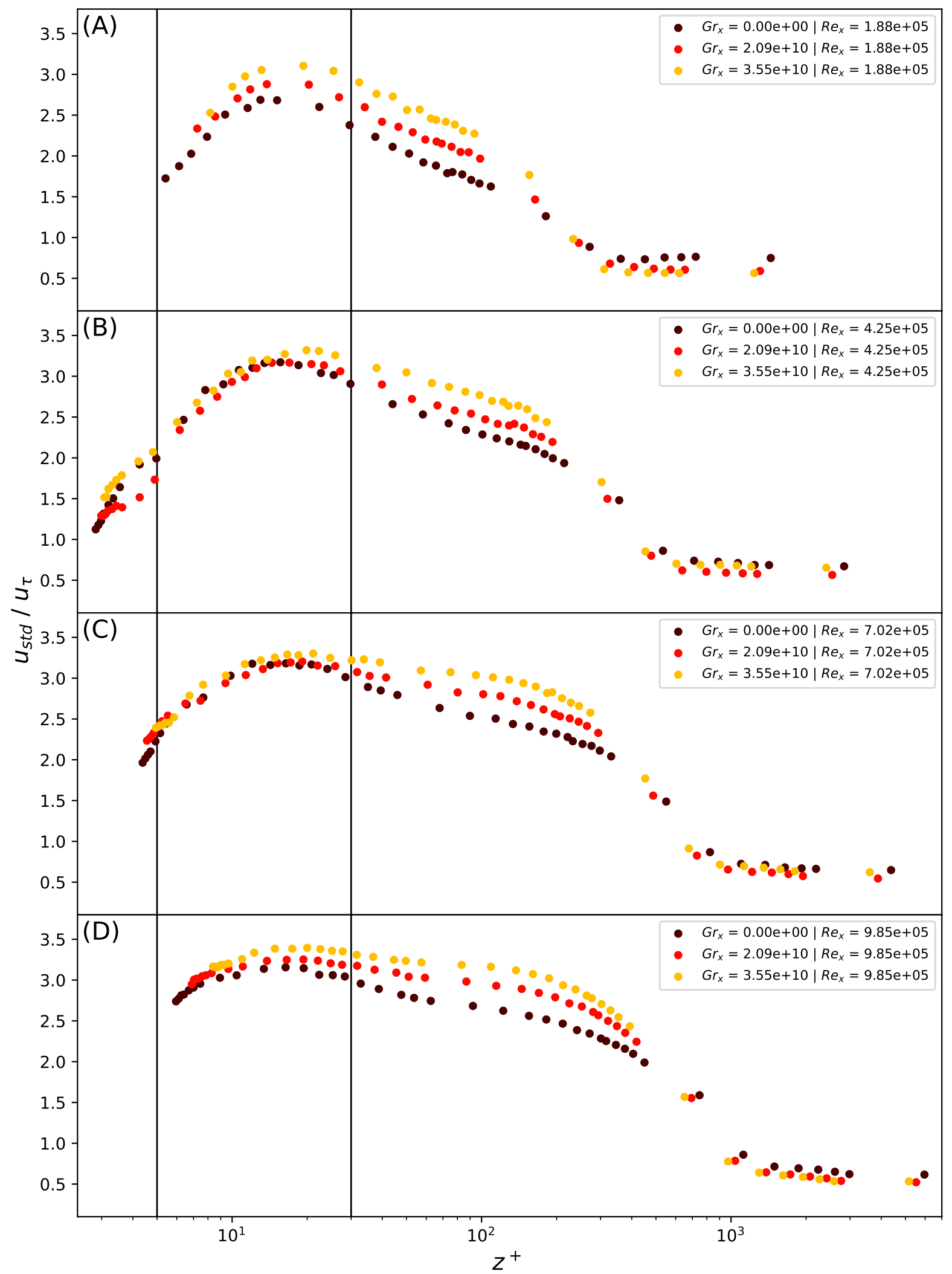

Figure 4.40: Standard deviation with shear velocity $u_{\tau}$ derived from Rodriguez 2015 [89] method 


\begin{tabular}{||c|c|c|c|c|c||}
\hline$R e_{x}$ & $G r_{x}$ & $u_{\tau}[\mathrm{m} / \mathrm{s}]$ & $\epsilon[\mathrm{m}]$ & $\delta[\mathrm{m}]$ & $\Pi$ \\
\hline \hline $1.88 \times 10^{5}$ & 0 & 0.055 & 0 & 0.091 & 0.290 \\
\hline $1.88 \times 10^{5}$ & $2.09 \times 10^{10}$ & 0.050 & $1 \times 10^{-5}$ & 0.081 & 0.676 \\
\hline $1.88 \times 10^{5}$ & $3.55 \times 10^{10}$ & 0.047 & $1 \times 10^{-4}$ & 0.072 & 0.890 \\
\hline $4.25 \times 10^{5}$ & 0 & 0.108 & 0 & 0.063 & 0.978 \\
\hline $4.25 \times 10^{5}$ & $2.09 \times 10^{10}$ & 0.096 & $1 \times 10^{-5}$ & 0.055 & 1.436 \\
\hline $4.25 \times 10^{5}$ & $3.55 \times 10^{10}$ & 0.091 & $1 \times 10^{-4}$ & 0.053 & 1.689 \\
\hline $7.02 \times 10^{5}$ & 0 & 0.166 & 0 & 0.058 & 1.068 \\
\hline $7.02 \times 10^{5}$ & $2.09 \times 10^{10}$ & 0.157 & $7 \times 10^{-5}$ & 0.053 & 1.556 \\
\hline $7.02 \times 10^{5}$ & $3.55 \times 10^{10}$ & 0.136 & $1.5 \times 10^{-4}$ & 0.052 & 1.979 \\
\hline $9.85 \times 10^{5}$ & 0 & 0.225 & 0 & 0.049 & 1.057 \\
\hline $9.85 \times 10^{5}$ & $2.09 \times 10^{10}$ & 0.209 & $1 \times 10^{-4}$ & 0.047 & 1.454 \\
\hline $9.85 \times 10^{5}$ & $3.55 \times 10^{10}$ & 0.195 & $2 \times 10^{-4}$ & 0.044 & 1.618 \\
\hline
\end{tabular}

Table 4.6: Table of fitting parameters for fig 4.38

Finally, with the newly calculated $u_{\tau}$ we also plot the velocity fluctuations $u_{s t d}$ as function of the wall distance $z^{+}$in fig. 4.40. Here, we observe that now we can align the maxima of the $u_{s t d}$ in the buffer region for different $\mathrm{Gr}_{x}$, with a very good agreement for some of the measurements done in a fully turbulent regime. The boundary layer size $\delta^{+}$is now clearly reduced and differences in the log region become now prominent for increasing Gr, with an overlap at the interface between wake and bulk.

This is a different approach to the analysis that we presented in sec. 4.2 . When compared to the observations introduced in previous sections this method does not produce a better collapse in the inner region, which means that either this method is not appropriate, or that heating changes the velocity field quantitatively compared to a purely shear-driven turbulent boundary. Note, that the buoyancy is very small compared to advective forces, e.g., $\mathrm{Ri}=0.04$ for $\mathrm{Gr}_{x}=3.55 \times 10^{10}$ and $\mathrm{Re}_{x}=9.85 \times 10^{5}$ and so is a priori not expected to affect much the streamwise velocity and the fluctuation intensity. The overlap in the buffer region appears to be solid, but a closer inspection confirms the observations in the previous sections. The increase of $\mathrm{Gr}_{x}$ in the fully turbulent boundary layer during our experiments always resulted in a more "advection" rich regime in the buffer region. The velocity profile inside the buffer region becomes log-like and cannot be fitted by the typically expected curve of the buffer region. The wake region cannot be successfully fitted by this method. A better fit in the wake region can only be obtained by changing the value of the von Karman constant, but this does not feel the right approach. 
All together the approach suggested by Rodriguez [89] is pointed at a fully turbulent boundary layer, with the goal to offer a better fitting method for low Reynolds numbers. This method has shown to be extremely effective in fitting our data when the boundary layer was not affected by the surface heat. The fit in the wake region is satisfying and the buffer region is well enough represented.

The fact that this method has not been effective in our application is not detrimental to the quality of the method. We have been able to obtain surprisingly good results in the fit of our data when $R e_{x} \geq 1.88 \times 10^{5}$ and $\mathrm{Gr}_{x}=0$. This result allows us to conclude that the increase of surface heat cannot be represented by the classical fitting quantities used in turbulent boundary layer models. Shear velocity $u_{\tau}$, boundary layer shift $\epsilon$, boundary layer size $\delta$, and wake parameter $\Pi$ are not enough to fully represent the transformations induced by the heat.

\subsection{Heat transport measurements}

\subsubsection{Local heat transfer under constant shear stress}

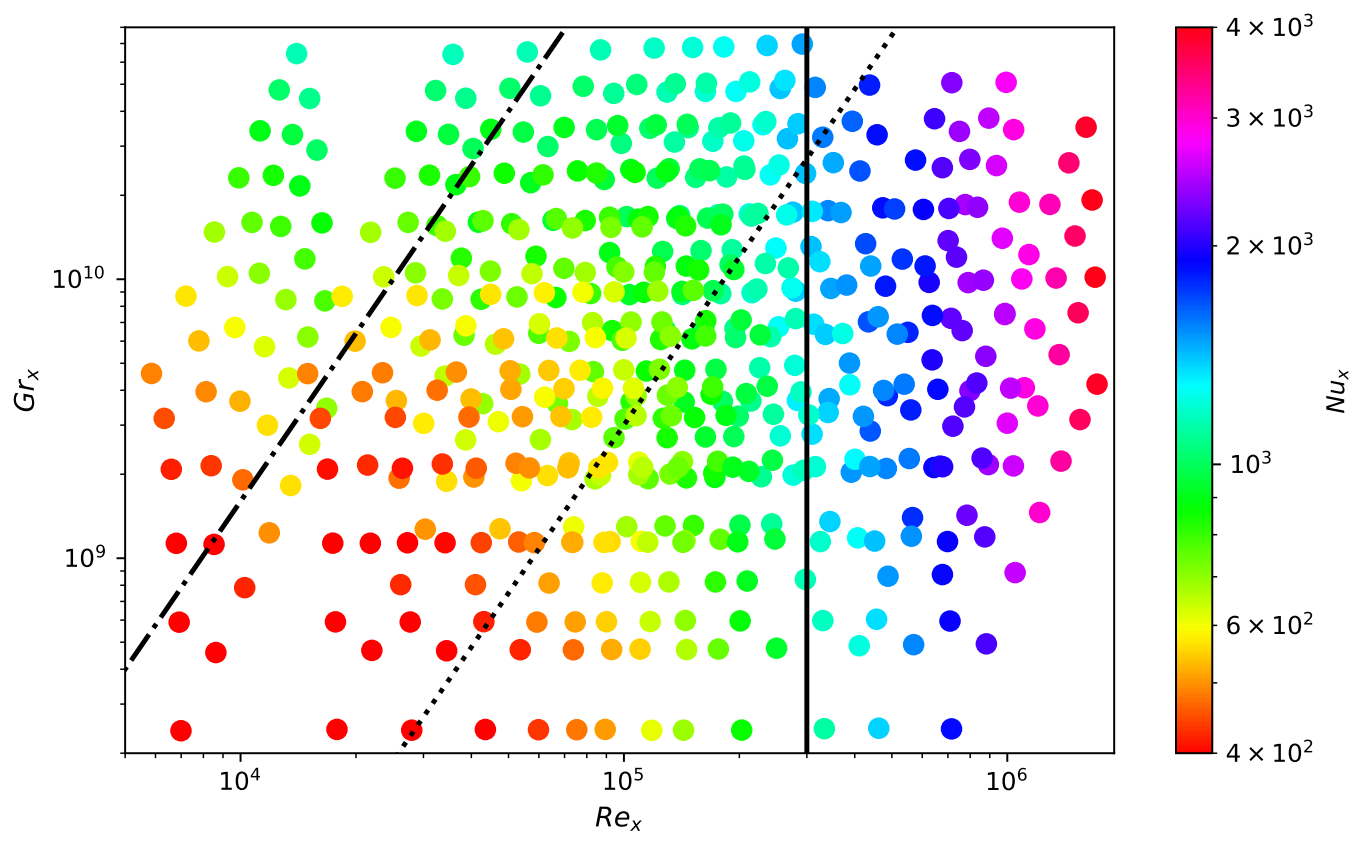

Figure 4.41: Parameter space covered by our measurements. Solid line represents expected laminar to turbulent transition of flat surface for $R e_{\text {crit }}=3 \times 10^{5}$, dash-dot line represent free to mixed convection transition with $R i=G r_{x} / R e_{x}^{2}=16$, dotted line represent mixed to forced convection transition with $R i=G r_{x} / R e_{x}^{2}=0.3$. 
In the previous chapter, we have focused our analysis on the structure and dynamics of the boundary layer itself. We have seen that heating from below had a clear effect on the velocity boundary layer and its characteristics. In this chapter, we will focus on the effect of those boundary-layer conditions on the heat transfer from the surface.

Our experiment has a 1-meter long adiabatic plate in front of the heated plate, and therefore the thermal boundary layer starts to develop also $1 \mathrm{~m}$ behind the velocity boundary layer, which needs to be taken into consideration for the development of scaling relationships between the control and response parameters.

In the presented work we use the distance from the leading edge $x$ as the typical length that is included in the control and response parameters, $G r_{x}, N u_{x}$ and $R e_{x}$. Our experimental setup, allows us to measure the heat transfer at 21 different locations distributed in 7 lines and 3 rows along the plate, where constant heat flux is applied from the bottom. ${ }^{2}$. Since we expect the time-averaged heat transfer to be constant along the spanwise direction, we average over the 3 rows in the following.

The freestream turbulence in our setup is smaller than $5 \%$. The two control parameters are the free flow velocity expressed as $R e_{x}$ and the difference between the free flow and the surface temperature expressed as $G r_{x}$, we define them as:

$$
R e_{x}=\frac{U_{0} x}{\nu}, \quad G r_{x}=\frac{g \beta\left(T_{w}-T_{0}\right) x^{3}}{\nu^{2}}
$$

Here, $g$ denotes the gravitational acceleration, $x$ the position along the plate with $x=0$ beginning of the adiabatic plate, $\beta$ the thermal expansion coefficient, $T_{w}$ the local surface temperature, $T_{0}$ the incoming flow temperature, and $\nu$ the kinematic viscosity.

The dimensionless parameter that we use to express the heat transfer is the well known Nusselt number that we define as:

$$
N u_{x}=\frac{q(x) x}{\lambda\left(T_{w}(x)-T_{0}\right)}
$$

with $\lambda$ being the thermal conductivity of air, and $q(x)$ the dimensional heat flux from the

\footnotetext{
${ }^{2}$ More details on the thermistor distribution can be found in chapter 3.2.1
} 
plate, calculated from temperature measurements inside the plate as:

$$
q(x)=-\frac{\lambda_{p}\left(T_{w}(x)-T_{b}(x)\right)}{H_{p}} .
$$

Here, $\lambda_{p}$ is the thermal conductivity of polycarbonate, $H_{p}$ the thickness of the polycarbonate layer, $T_{w}(x)$ the local temperature of the top aluminium plate, and $T_{b}(x)$ the local temperature of the bottom aluminium plate. A more detailed schematic of the plate was presented in chapter 3.2 .

In figure 4.41, we show an overview of the data that we have collected in a $\operatorname{Re}_{x^{-}}-\mathrm{Gr}_{x^{-}}$ parameter space with colors representing the heat flux $\left(N u_{x}\right)$. The color distribution already suggest that $N u_{x}$ increases both with increasing $R e_{x}$ and $G r_{x}$. In total, we have acquired 511 different data points. These data have been collected at 7 different locations above the heated plate for different combinations of velocity $U_{0}$ and temperature $\Delta T$.

Since the fluid properties vary with temperature, and since strong vertical temperature gradients occur in the flow, the question arises at which temperature we shall evaluate the fluid properties for the calculation of $\mathrm{Gr}_{x}$ and $\mathrm{Re}_{x}$. We decide to evaluate all fluid properties at the local surface temperature $T_{w}(x)$. One might argue that a temperature somewhere between $T_{0}$ and $T_{w}$ would be more suitable, but since the boundary layer is not symmetric a simple average of both values is probably not a good choice either and hence for simplicity we decided on $T_{w}(x)$. We note in this regard that the viscosity only plays a role in the close vicinity of the wall and hence at least for the calculation of $\nu, T_{w}$ is a good choice.

In fig. 4.42 we present the local heat flux $N u_{x}$ as function of the local Reynolds number $R e_{x}$. In general, $N u_{x}$ increases with increasing $R e_{x}$ and is presented to us in a funnel-like distribution with the $N u_{x}$ asymptotically approaching a power law $N u_{x} \propto R e_{x}^{\alpha}$ for the largest $\operatorname{Re}_{x}$. When the surface temperature $G r_{x}$ is small (blue), an increase of $\operatorname{Re}_{x}$ leads to an increase of $\mathrm{Nu}_{x}$ already at the smallest $\mathrm{Re}_{x}$.

The slope in this log-log representation, i.e., the effective exponent $\alpha_{e f f}=\partial\left(\log \mathrm{Nu}_{x}\right) / \partial\left(\log \operatorname{Re}_{x}\right)$, increases with increasing $\operatorname{Re}_{x}$ and reaches $\alpha_{e f f} \rightarrow \alpha$ for sufficiently large $\operatorname{Re}_{x}$. We have shown in sec. 2.4.2 that when buoyancy is neglected (forced convection) and the boundary layer is fully turbulent, we expect a scaling of $\alpha=0.8$. Indeed, a fit to the data with the smallest influence of buoyancy and the largest shear stress, Richardson numbers 


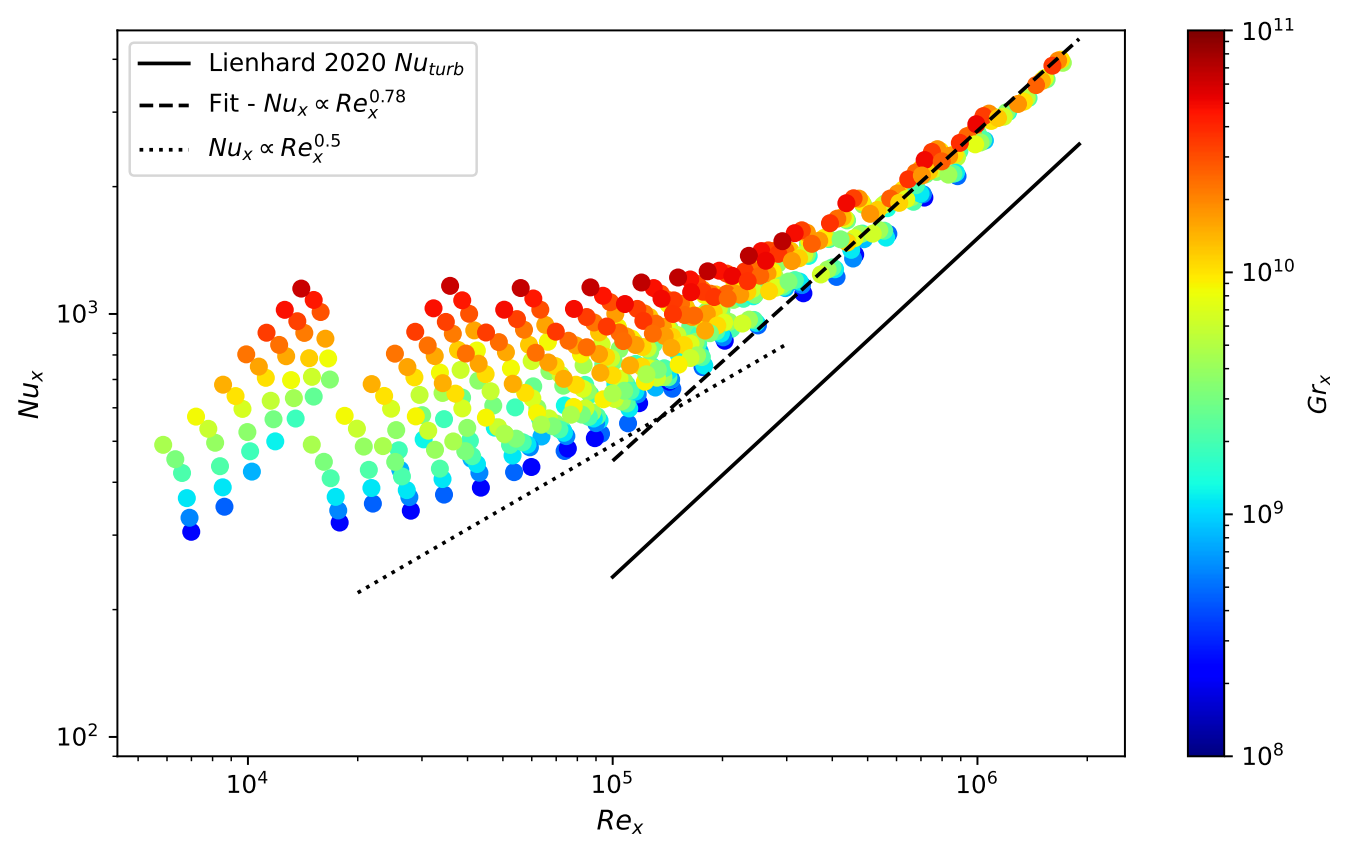

Figure 4.42: Heat transfer $\left(N u_{x}\right)$ as function of the Reynolds number Re $e_{x}$. Color code represents Grashof number $G r_{x}$. Dashed line marks a power-law fit to the data with $R i_{x} \leq 10^{-2}$ (forced convection), resulting in an exponent $\alpha=0.78$. The solid line marks predictions for a fully turbulent boundary layer from Lienhard eq. 6 [90]. The dotted line marks a power law with exponent 1/2, as expected for a laminar boundary layer without buoyancy.

$\mathrm{Ri} \leq 10^{-2}$, results in a close value $\alpha_{f i t}=0.78 \pm 0.01$. When $G r_{x}$ is high (red), and $\mathrm{Re}_{x}$ is small, $\mathrm{Nu}_{x}$ is independent of $\mathrm{Re}_{x}$ for up to a decade. This is the free convection regime where the buoyancy dominates the heat transfer. Only for sufficiently large $\operatorname{Re}_{x}$ do we observe an increase in the heat transfer due to increasing shear forces. Then, with increasing $\operatorname{Re}_{x}$, also the data for the largest surface temperature converge asymptotically to $N u \propto R e_{x}^{\alpha}$, the regime of forced convection.

We see already in fig. 4.42 a slightly disturbing feature, namely that different $\mathrm{Nu}_{x}$ are sometimes observed at the same control parameters $\operatorname{Re}_{x}$ and $\mathrm{Gr}_{x}$. Likewise, we see we observe at a given $\mathrm{Re}_{x}$ the same $\mathrm{Nu}_{x}$ at different $\mathrm{Gr}_{x}$. The reason for this is that we use the distance from the leading edge $x$ as a length scale for our dimensionless parameters. It is a priori not clear that the same relation $\mathrm{Nu}_{x}\left(\mathrm{Gr}_{x}\right)$ at a given $\mathrm{Re}_{x}$ hold if one changes the inflow velocity $U_{0}$, the heat supply to the bottom plate, or the measurement location $x$. In fact, $\Delta T(x)$ and hence $\mathrm{Gr}_{x}$ are technically responses parameters for any position $x \neq 1.18 \mathrm{~m}$, i.e., the location of the thermistor line at which we fix the temperature difference. We will discuss below whether one can find a better length scale that helps to 
better collapse the data.

In this regard, we also remind the reader that there is a $1 \mathrm{~m}$ long adiabatic plate in front of the heating section. While the velocity boundary layer starts to develop at $x=0$, a thermal boundary layer only starts to develop at $x=1.00 \mathrm{~m}$. While there are models in the literature to correct for such an adiabatic plate in front, they usually only consider laminar boundary layers and very short plates.

We compare our data to theoretical predictions (eq. 2.42, see [90]), for a fully turbulent boundary layer, shown as a solid line in fig. 4.42. We see that our data follow asymptotically the scaling, they have clearly larger $\mathrm{Nu}_{x}$. We believe that this discrepancy can be explained with the existence of the adiabatic plate. Clearly, the heat flux at the beginning of the heated plate must be enhanced in our case, because the inflow is not preheated as would be the case if also the first $1 \mathrm{~m}$ section is heated as in the model.

In fig. 4.42 we also show a dotted line $\mathrm{Nu}_{x} \propto \mathrm{Re}_{x}^{1 / 2}$, which represents the heat flux through a laminar boundary layer when buoyancy is neglected. We show this line for comparison but point out again that the boundary layer is never really laminar, even for the smallest $\operatorname{Re}_{x}$. When the effective exponent $\alpha_{\text {eff }}$ reaches values close to 0.5 at around $\operatorname{Re}_{x} \approx 10^{5}$ for the blue point (the smallest $\mathrm{Gr}_{x} \approx 2 \times 10^{8}$ ), this is caused by the decreasing influence of buoyancy when $\mathrm{Re}_{x}$ increases.

Different experimental studies characterize the scaling of the heat transfer $N u_{x}$ against the shear flow $R e_{x}$ i.e. Sugawara in 1951 [91], Reynolds in 1958 [92-95], Wang in 1982 [96]. Sugawara [91] has been one of the first that obtained a scaling for the laminar boundary layer $N u \propto R e_{x}^{0.5}$ and $N u \propto R e_{x}^{0.8}$ for turbulent ones. These scaling are estimated and measured in a forced convection regime, where buoyancy plays only an insignificant role. We do not observe in our data any $N u \propto R e_{x}^{0.5}$ scaling, in our experiment we have not observed laminar boundary layer in the range of our measurements, the $N u \propto R e_{x}^{0.5}$ scaling is expected to be observed in forced convection regime and without an adiabatic plate.

In fig. 4.43 we only consider measurements taken at $x=1.18 \mathrm{~m}$ away from the leading edge, to look at the scaling of the $N u_{x} \propto G r_{x}$. Qualitative similar results have also been observed for all the other thermistor lines with only a change in total magnitude.

We first consider $\mathrm{Nu}_{x}$-data acquired at the smallest $\mathrm{Re}_{x} \leq 2 \times 10^{4}$ (blue in fig. 4.43) and large $\operatorname{Gr}_{x} \geq 10^{9}$. In these cases, the buoyancy was largest compared to advective 


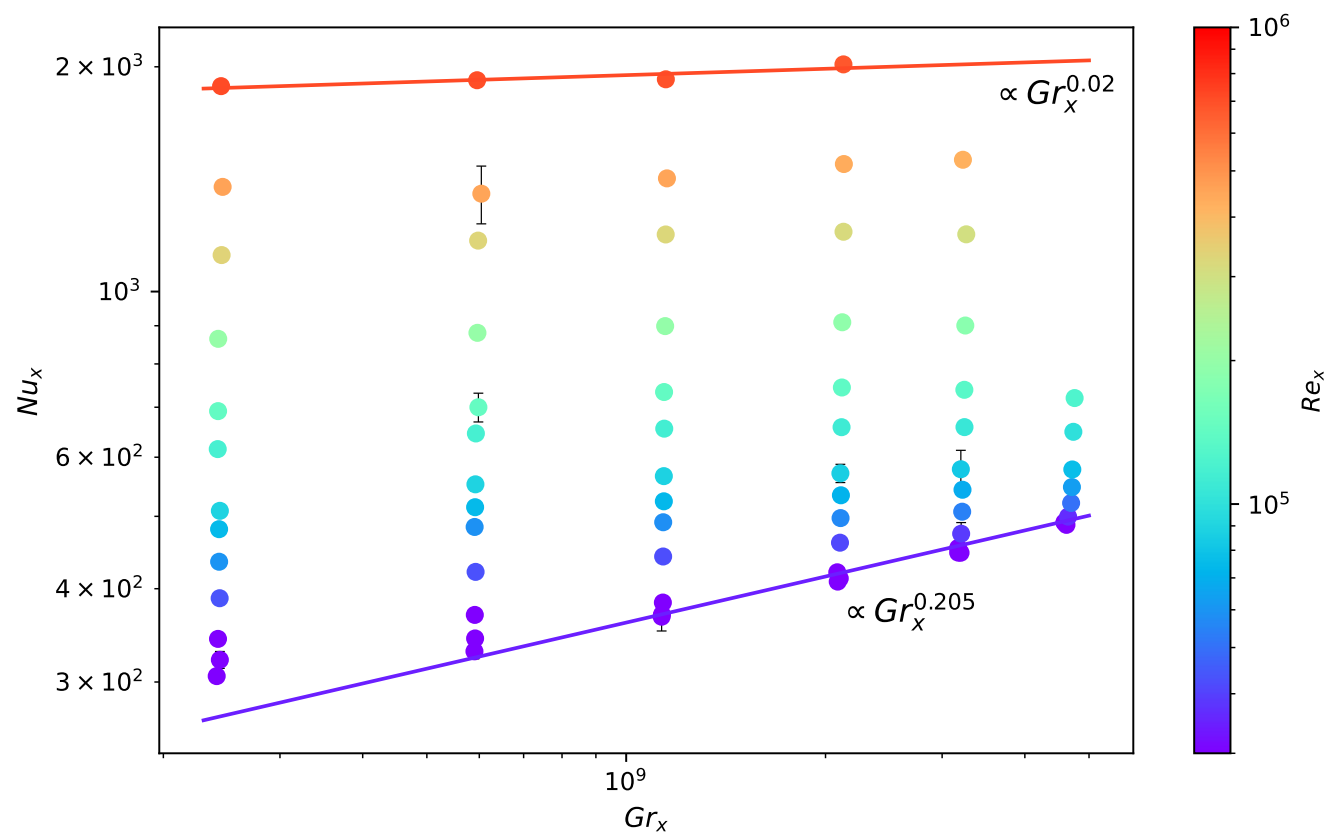

Figure 4.43: Heat transfer scaling as function of the Grashof number, the $N u_{x}$ and $G r_{x}$ are calculated at Line 1 thermistors. Colors code represent Reynolds number. Error-bars represent maximum estimated error over $N u_{x}$. Fit parameter has been obtained fitting $R e_{x} \leq 2 \times 10^{4}$ and $G r_{x} \geq 1 \times 10^{9}$.

forces so the flow can be considered as natural convection. A power law fit to these data results in a scaling $N u_{x} \propto G r_{x}^{0.205 \pm 0.008}$ (purple line in fig. 4.43). We observe a strong decrease of the effective exponent $\partial\left(\log N u_{x}\right) / \partial\left(\log G r_{x}\right)$ with increasing $R e_{x}$, down to a value of $\mathrm{Nu}_{x} \propto \mathrm{Gr}_{x}^{0.02}$ for the largest $\mathrm{Re}_{x}$ (red line in fig. 4.43). The latter result is expected because we have seen already in fig. 4.42 that at sufficiently large $\mathrm{Re}_{x}$, buoyancy is negligibly small and hence the heat transport becomes independent of $\mathrm{Gr}_{x}$.

Goldstein in 1982 [97] presented a collection of experimental results where he observed a set of powerlaws to correlate heat transfer $N u$ and Rayleigh number $R a$ in the free convection regime, with scaling between $N u \propto R a^{1 / 4}$ and $N u \propto R a^{1 / 5}$. His results were taken at a fixed $\operatorname{Pr}=0.7$ and since the Rayleigh number $\mathrm{Ra}$ and Grashof number are related $R a_{x}=G r_{x} \cdot P r$, his results can be compared directly compared to ours and are in very good agreement.

The ratio between buoyancy and advection forces is usually described by the Richardson number Ri. For small Ri buoyancy can be neglected and temperature is advected as a passive scalar. This regime is referred to as forced convection. For very large Ri, buoyancy dominates over advection forces due to the mean flow. This regime is called 
free or natural convection.

Sparrow in 1959 [98] characterized the convection regimes as function of the Richardson number:

$$
R i_{x}=\frac{G r_{x}}{R e_{x}^{2}}
$$

The study suggests some regions in which we do expect a transition from one regime to another.

- For $R i<0.3$ the convection is said to be Forced, with the shear forces dominant over the buoyant forces.

- For $0.3<R i<16$ the convection is said to be Mixed, with the buoyant forces and shear forces comparable.

- For $R i>16$ the convection is said to be Free, with the buoyant forces dominant over the shear forces.

It is worth noticing that in literature there are also some different $G r / R e^{\alpha}$ scaling depending on the application and specific needing of those works (see e.g., [96, 99-101]).

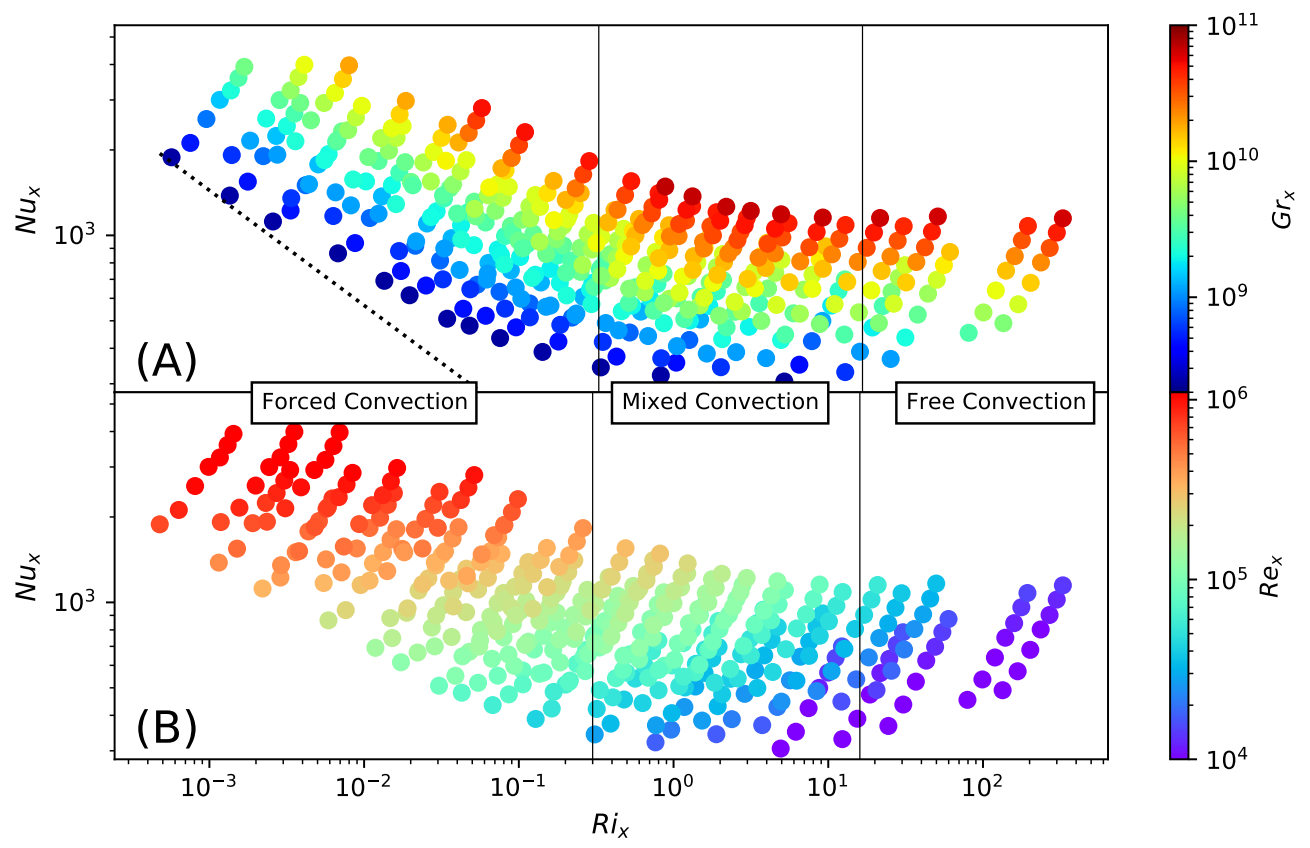

Figure 4.44: Nusselt number scaling as function of the Richardson number. Vertical solid lines at $R i_{x}=0.3$ and $R i_{x}=16$ mark the boundaries between the forced, mixed and free convection regimes as suggested by [98]. Dotted line represent $N u \propto R i^{-2 / 5}$ scaling. 
Figure 4.44 shows $\mathrm{Nu}_{x}$ plotted as a function of $\mathrm{Ri}_{x}$. In this figure, we mark and label the regimes of forced, mixed and free convection as suggested by [98]. Figure 4.44(A and B) present the same data, but while the color of the symbols in (A) represents the $\mathrm{Gr}_{x}$, the color in (B) represent $\operatorname{Re}_{x}$. We also show in fig. 4.44(A) as dotted line a power law $\propto \mathrm{Ri}_{x}^{-2 / 5}$, which equals for constant $\mathrm{Gr}_{x}$ to $\propto \mathrm{Re}_{x}^{4 / 5}$ as observed for the turbulent forced convection regime. The transition from forced to free convection is clearly visible for a given $\mathrm{Gr}_{x}$. In the forced convection regime $\left(\operatorname{small} \mathrm{Ri}_{x}\right) \mathrm{Nu}_{x}$ decrease sharply, in the free convection regime (large $\mathrm{Ri}_{x}$ ), $\mathrm{Nu}_{x}$ is rather independent on $\mathrm{Ri}_{x}$. However, we also see that the change of the monotonic behaviour, i.e., the $\mathrm{Ri}_{x}$ where forced convection ends and mixed convection starts is not the same for different $\mathrm{Gr}_{x}$, and the transitional $\mathrm{Ri}_{x}$ suggested by Sparrow [98] does not hold for all $\mathrm{Gr}_{x}$. Instead, the flat $\mathrm{Nu}_{x}$-plateau is reached for smaller $\mathrm{Gr}_{x}$ also at smaller $\mathrm{Ri}_{x}$.

Lower surface temperature results in a more sudden and later transition to forced convection. Figure (B) is presented for completeness, shear stress distribution do not present surprising findings.

\subsubsection{New length-scales and power-laws}

One of the main goals for using dimensionless quantities is to reduce the number of control parameters and then to find simple scaling relations between the remaining control parameters and the response parameters of interest. These parameters depend on a typical length scale, which is of utmost importance for the collapse of the data sets to facilitate comparison. So far, we have used $\mathrm{Gr}_{x}, \mathrm{Re}_{x}$, and $\mathrm{Nu}_{x}$, where the typical length involved in their calculation was the distance from the leading edge of the surface $x$.

We saw, that in the regime of forced convection, $x$ as a length scale works well so that the heat transfer $\mathrm{Nu}_{x}$ is only a function of $\mathrm{Re}_{x}$ (see fig. 4.42). Clearly, when buoyancy plays a role for mixed and free convection, $\mathrm{Nu}_{x}$ also depends on $\mathrm{Gr}_{x}$. Hence, a proper rescaling of $\mathrm{Nu}_{x}, \mathrm{Gr}_{x}, \mathrm{Re}_{x}$ is desired so that all data collapse on a single master curve.

A priori one would think that it is difficult to find rescaled parameters so that data collapse on a low-dimensional curve. This is because there are different mechanisms involved with different scalings. The boundary layer can not only be laminar or turbulent, with different scalings, with the turbulent boundary layer scaling that can be caused by 


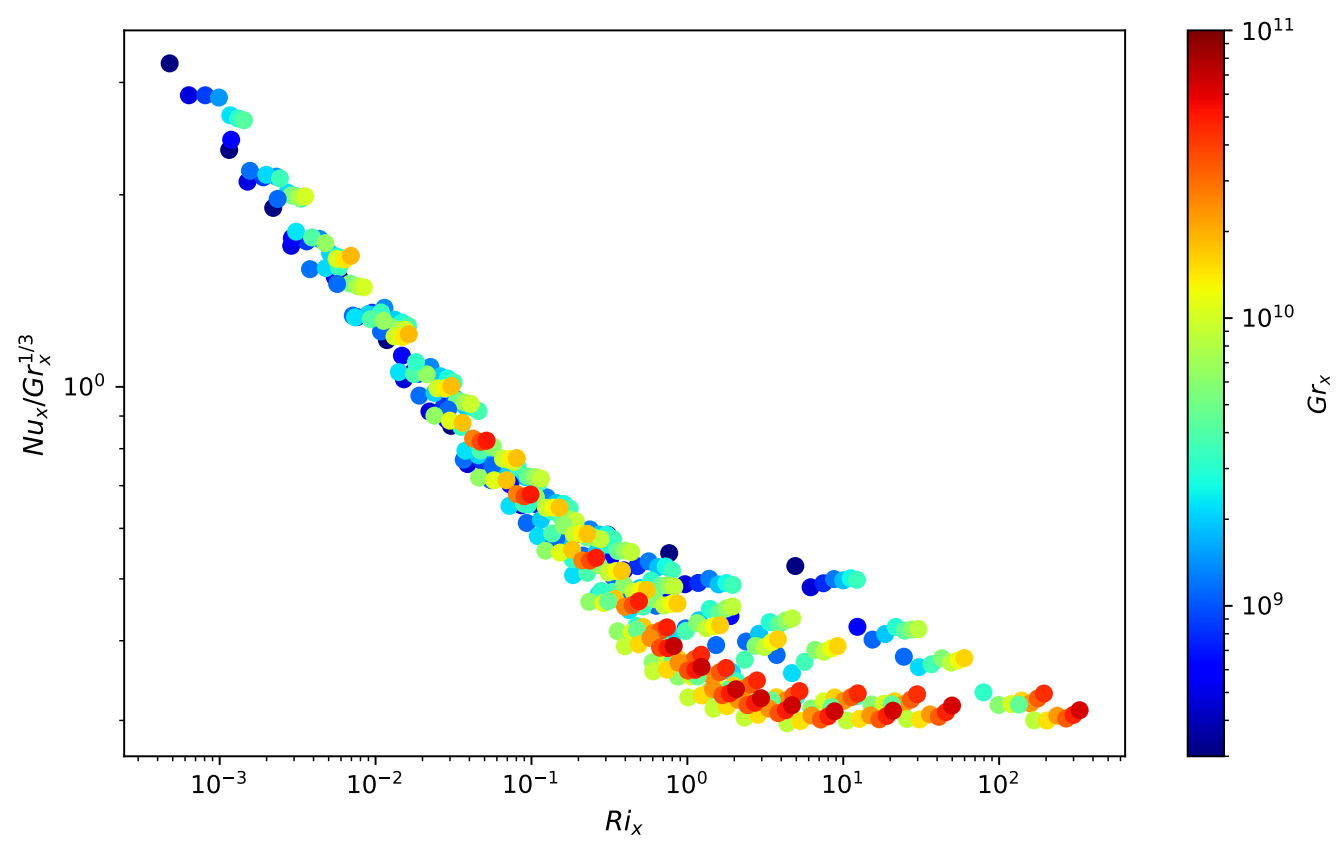

Figure 4.45: Rescaled Nusselt number $N u_{x} / G r_{x}^{1 / 3}$ as function of the Richardson number $R i_{x}$ for different $\mathrm{Gr}_{x}$ (color code).

buoyancy, by shear or by an interaction of both. However, now we want to try out different rescalings in the hope for a better collapse of the data at least in some regions, which would help to build simplified scaling models.

While the Richardson number $R i_{x}=\frac{G r_{x}}{R e_{x}^{2}}$ is a good candidate for such a rescaling, as shown in fig. 4.44, we do not obtain an unambiguous correlation between $R i_{x}$ and $N u_{x}$. Above, we have already discussed that $x$ might not be a proper length scale that is relevant in our system. Therefore, let's first try to remove the heat flux dependency on it by rescaling the Nusselt number as $\frac{N u_{x}}{G r_{x}^{13}}$.

This new approach results in a satisfying collapse for the fully forced region, and a partial collapse in the mixed and free convection regions.

We can consider the Grashof number as the ratio between acceleration time scales caused to buoyancy and the time scale related to viscous damping. Because buoyancy always acts normal to the gravity (wall-normal direction), one would assume a vertical length scale rather than horizontal to be more appropriate. We do believe that a good length scale could be related to the boundary layer height $\delta(x)$. Due to the lack of adequate models to calculate the turbulent boundary layer height when heat is applied, we will assume the scaling of the boundary layer to be similar to the one solely driven by 


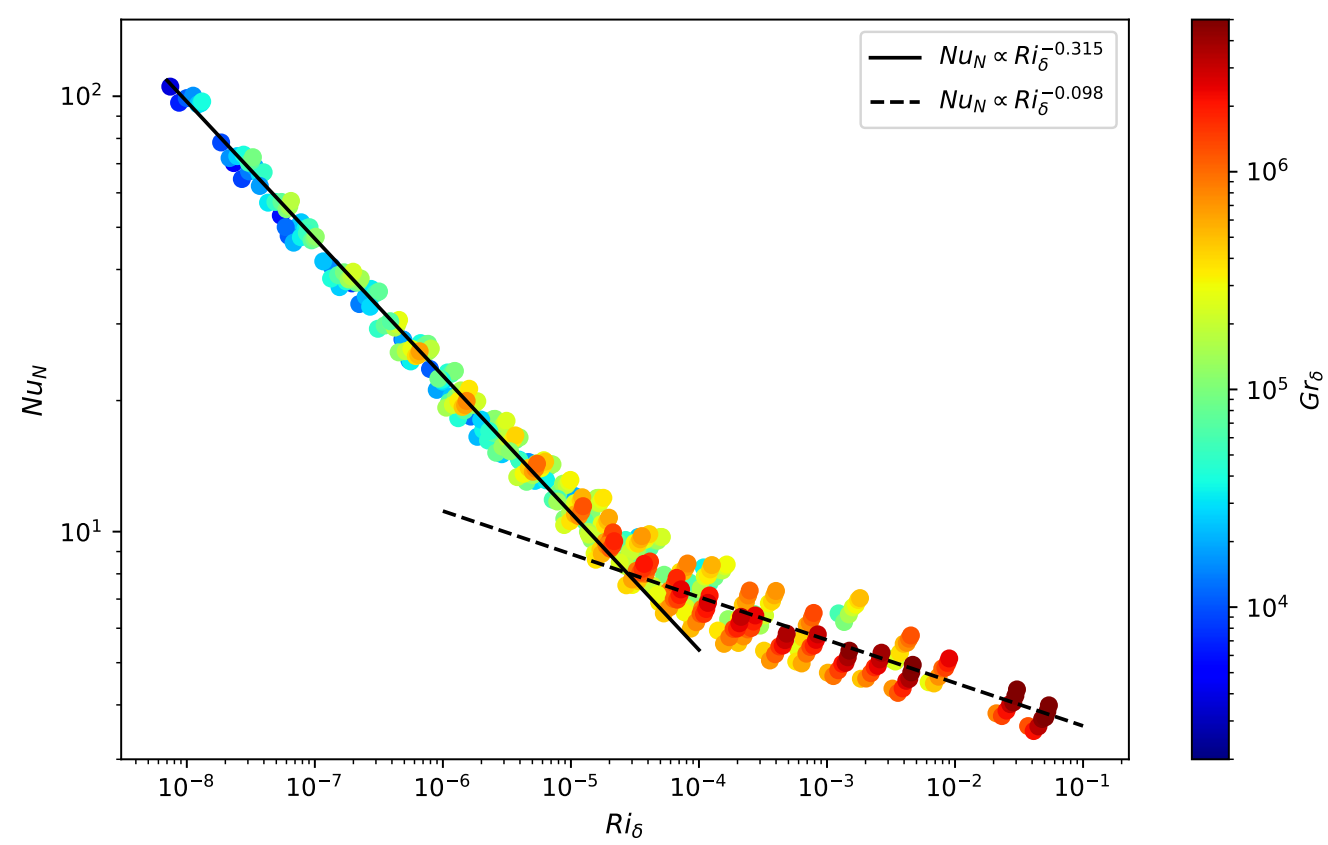

Figure 4.46: Newly defined Nusselt number $N u_{N}$ as function of Richardson number based on the boundary layer height Ri $i_{\text {. }}$ Data points are color coded using the new Grashof number Gr. The solid and dashed straight lines are power law fits to the regime of shear dominated convection $\left(R i_{\delta}<3 \times 10^{-5}\right)$ and the regime of buoyant dominated convection $\left(R i_{\delta}>8 \times 10^{-5}\right)$.

shear stress. To estimate the latter for the fully turbulent case, we use eq. 2.24 which was introduced already in sec. 2.3.2:

$$
\delta \approx \frac{0.37 x}{R e_{x}^{1 / 5}}
$$

With this, we define new dimensionless parameters:

$$
G r_{\delta}=\frac{g \beta \Delta T \delta^{3}}{\nu^{2}}, \quad R i_{\delta}=\frac{G r_{\delta}}{R e_{x}^{2}}, \quad N u_{N}=\frac{N u_{x}}{G r_{\delta}^{0.35}}
$$

and can reanalyse our data.

In fig. 4.46 we present the same dataset that we have already shown above in fig. 4.45 but now with the newly defined $\mathrm{Nu}_{N}$ as function of $\mathrm{Ri}_{\delta}$. Data plotted in this way now show both a good collapse in the forced convection regime, as expected, but also a significantly better collapse of the data for different $\mathrm{Gr}_{\delta}$ in the regimes of mixed and natural convection for large $\mathrm{Ri}_{\delta}$. The data plotted in this way show two regions that can be fit using different power-laws with a transition at $\mathrm{Ri}_{\delta} \approx 2 \times 10^{-5}$ between them. For the low $\mathrm{Ri}_{\delta}$ range, fitting $R i_{\delta}<3 \times 10^{-5}$ we obtain a scaling of $N u_{N} \propto R i_{\delta}^{-0.315 \pm 0.002}$. This scaling is equivalent to $\mathrm{Nu}_{x} \propto \mathrm{Gr}_{\delta}^{0.035} \operatorname{Re}_{x}^{0.63}$, which is as expected for the forced convection regime, 


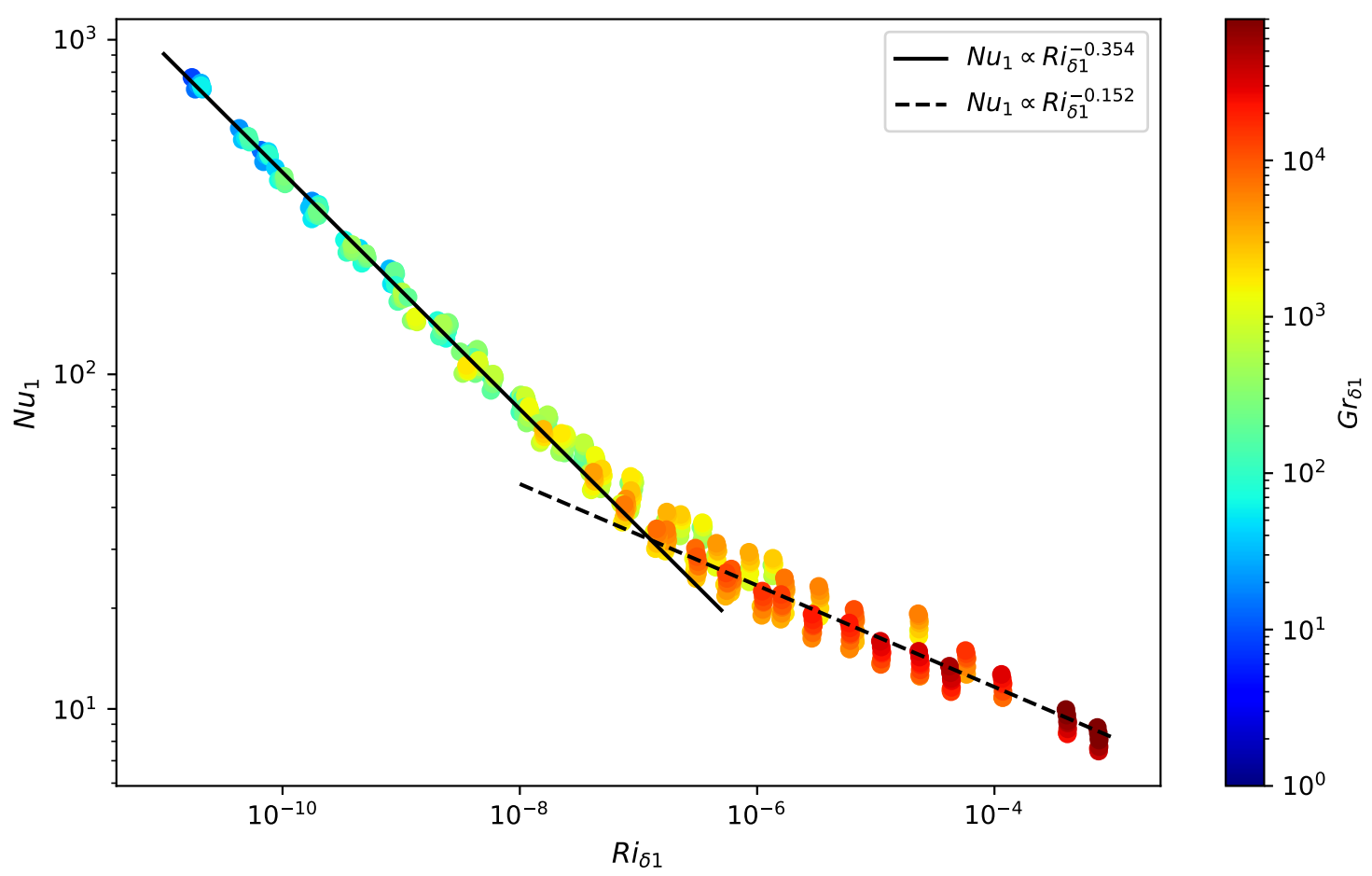

Figure 4.47: Newly defined Nusselt number $N u_{1}$ as function of Richardson number based on the boundary layer height $R i_{\delta 1}$. Data points are color coded using the new Grashof number Gr $r_{\delta 1}$. The solid and dashed straight lines are power law fits to the regime of shear dominated convection $\left(R i_{\delta 1}<1 \times 10^{-7}\right)$ and the regime of buoyant dominated convection $\left(R i_{\delta 1}>1 \times 10^{-6}\right)$

a very small $\mathrm{Gr}_{\delta}$-dependency. The $\mathrm{Re}_{x}$-exponent is somehow smaller than the original fitted $\alpha=0.78$, which we attribute to the fact that we have also used data with larger $\mathrm{Gr}_{\delta}$ for the fit here, compared to the fit above. For the larger $\mathrm{Ri}_{\delta}$, fitting $R i_{\delta}>8 \times 10^{-5}$ we obtain $N u_{N} \propto R i_{\delta}^{-0.098 \pm 0.007}$. This result can be expressed in the $x$-based parameters as

$$
\mathrm{Nu}_{x} \propto \mathrm{Gr}_{x}^{0.252} \operatorname{Re}_{x}^{0.196}\left(\frac{\delta}{x}\right)^{0.756} \propto \mathrm{Gr}_{x}^{0.252} \operatorname{Re}_{x}^{0.045}
$$

The $\mathrm{Gr}_{x}$-exponent is somehow similar to the previously found in fig. 4.43, but also not have a significant $\operatorname{Re}_{x}$ dependency. The new scaling method helps to better collapse the data.

We also note that when the data are plotted in this way, they show a seemingly sharper transition between the forced and free convection regime. This suggests that the new length scale better represents the growth of the buoyant force contribution to the heat transfer as a function of $\mathrm{x}$. In short, we believe that this rescaling is promising in order to characterise the transition between forced and free convection. This method has 
shown to work well in the shear dominated regime, but also result in an improved collapse when buoyant force is dominant.

For the definition of $\mathrm{Ri}_{\delta}$ and $\mathrm{Gr}_{\delta}$, we have assumed the eq. 2.24, which estimates the boundary layer height when it is fully turbulent. While this approach works decently well, we know from velocity measurements that the boundary layer was not fully turbulent for $\operatorname{Re}_{x} \leq 1.88 \times 10^{5}$ but rather in a transitional regime (see sec. 4.1.3). Measurements at such small $\mathrm{Re}_{x}$ correspond to larger $\mathrm{Ri}_{\delta}$ (free and mixed convection regime) and one might speculate whether a different estimate for $\delta$ would improve the collapse of the data for different $\mathrm{Gr}_{\delta}$ at larger $\mathrm{Ri}_{\delta}$.

Keeping in mind that this will be a more speculative approach, we can increase the scaling exponent for the relation between $\delta$ and $\operatorname{Re}_{x}$ to 0.35 . We choose this value because it resulted in the best collapse also for larger $\mathrm{Ri}_{\delta}$. However, we note that this value falls somewhere in between the scaling for a turbulent boundary layer height $\delta \propto R e_{x}^{0.2}$ and the scaling of a laminar boundary layer $\delta \propto R e_{x}^{0.5}$. Long story short, we define

$$
\delta 1 \approx \frac{0.37 x}{R e_{x}^{0.35}} \text { and } \quad N u_{1}=\frac{N u_{x}}{G r_{\delta 1}^{0.41}}
$$

and re-plot our data in fig. 4.47.

The data plotted in this way show again two regions that can be fitted using different power-laws with a transition at $\operatorname{Ri}_{\delta 1} \approx 1.2 \times 10^{-7}$ between them. For the range of $R i_{\delta 1}<1 \times 10^{-7}$ we obtain a scaling of $\mathrm{Nu}_{1} \propto R i_{\delta 1}^{-0.354 \pm 0.002}$. This scaling is equivalent to $\mathrm{Nu}_{x} \propto \mathrm{Gr}_{\delta 1}^{0.056} \operatorname{Re}_{x}^{0.708}$, which is again expected for the forced convection regime, with a very small $\mathrm{Gr}_{\delta 1}$-dependency. The $\mathrm{Re}_{x}$-exponent is here closer to the original fitted $\alpha=0.78$. This change can be attributed to the better collapse obtained in the shear driven region also for data obtained at high $G r_{x}$. For the larger $\mathrm{Ri}_{\delta 1}$, fitting a power law to the data with $R i_{\delta}>1 \times 10^{-6}$ results in $N u_{N} \propto R i_{\delta}^{-0.152 \pm 0.008}$. This result can be expressed in the $x$-based parameters as

$$
\mathrm{Nu}_{x} \propto \mathrm{Gr}_{x}^{0.258} \operatorname{Re}_{x}^{0.304}\left(\frac{\delta}{x}\right)^{0.774} \propto \mathrm{Gr}_{x}^{0.258} \operatorname{Re}_{x}^{0.033}
$$

The $\mathrm{Gr}_{x}$-exponent again is somehow similar to the previously found in fig. 4.43, and the $\operatorname{Re}_{x}$ dependency is small as expected for the free convection regime. This scaling helps us 
to improve even further the data collapse presented in fig.4.46. The improvement obtained with the introduction of the new definitions $\delta 1$ and $N u_{1}$ may suggest the opportunity to refine even further the approach, but at the moment we are not able to offer a valid explanation for the shown improvement.

All together it seems reasonable to consider the new proposed length scale $\delta$, and the new defined $N u_{N}$ as an improvement over the previous solution along the entire range of applications. We have been positively surprised by the results obtained with this collapse method and we feel that this new way to collapse the data allows for a better modeling and therefore predicting ability. We want to point that is of course necessary to test this result on dataset that span larger velocity, length and temperature ranges in order to test its validity as a general approach. This new proposed approach is an important step forward in the modelling of the heat transport in mixed convection regime, which then only depends on a single parameter for different convection regimes. We hope that our study can encourage other scientists to look at this regime that we feel is still not well understood, in an attempt of improving the general understanding and refine models and theory for the evolution of the buoyant force along the surface.

\subsection{Thermal boundary layer profile}

In this last section of this chapter, we present some preliminary results of temperature profile measurements. We define $T_{w}(x)$ as the surface temperature and $T_{0}$ as the outer flow temperature. All temperature data are collected at $x=2.35$ and different $z$. As usual, we keep $U_{0}$ constant during an entire measurement, as well as $\Delta T$ that we define as difference in temperature of the first row of thermistors $\left(T_{L 1}\right)$ and the inflow temperature $\left(T_{0}\right)$ :

$$
\Delta T=T_{L 1}-T_{0}
$$

We use the vertical thermistor rod described in sec. 3.5 in order to measure the temperature at 12 different vertical positions for about 14 hours or so with a sampling frequency of $0.2 \mathrm{~Hz}$ frequency. With $T(z)$ being the time average temperature at height $z$, we define:

$$
\Theta(z)=\frac{T(z)-T_{w}}{T_{0}-T_{w}}
$$


Figure 4.48(A) shows measurements of $\Theta$ for the fastest flow $\left(\operatorname{Re}_{x}=1.44 \times 10^{6}\right)$ and three different $\mathrm{Gr}_{x}$. The data look peculiar. Most importantly, the temperature profile is highly non-monotonic, in particular for the blue data points that represent the smallest $\mathrm{Gr}_{x}$. For these data, $\Theta$ increases steeply for small $z$, but reaches a maximum at maybe $z \approx 7 \mathrm{~cm}$ or so. From there on $\Theta$ decreases with increasing $z$ up to $\mathrm{z}=50 \mathrm{~cm}$, where it reaches a minimum. After that $\Theta$ increases again and reaches values $\Theta>1$ for the largest $\mathrm{z}$ at $1.1 \mathrm{~m}$ from the surface. Interestingly, the maxima at $\approx 7 \mathrm{~cm}$ occurs where we expect the end of the velocity boundary layer. The minima is roughly in the bulk of the wind tunnel and the last point is at the upper end of the wind tunnel.

This non-monotonic behaviour is puzzling as we would have expected a sharp increase of $\Theta$ close to the surface but then slope decreases so that $\Theta$ asymptotically reaches $\Theta=1$ towards the end of the boundary layer. First of all, one needs to consider that for the blue points the temperature difference was only $\Delta T=1 \mathrm{~K}$ and hence variation clearly visible are very small. The temperature difference between the $\Theta$-minimum in the vertical center of the wind tunnel $(\mathrm{z}=0.5 \mathrm{~m})$ and close to the top $(\mathrm{z}=1.1 \mathrm{~m})$ is only $0.15 \mathrm{~K}$. In other words, the bulk flow is $150 \mathrm{mK}$ warmer than the top boundary. We believe that this can easily be explained by heating of the flow solely due to viscose dissipation. The top of the tunnel has nearly the same temperature as the laboratory environment (wind tunnel walls are very thin metal sheets), but the air that is sucked in on one side of the wind tunnel that gets accelerated by the propeller, and squeezed through the laminarizing grid, certainly has to heat up due to energy dissipation. Next to this viscose dissipation, there is also friction of the bearings of the propeller. For a mass flux through the tunnel of about $10 \mathrm{~kg} / \mathrm{s}$, roughly $1.5 \mathrm{~kW}$ of energy must be dissipated to heat up the air by $150 \mathrm{mK}$. The engine that drives the propeller has a maximal power of $30 \mathrm{~kW}$.

To account for this effect, we want to redefine the reference temperature $T_{0}$. Instead of using the temperature at the adiabatic plate, from now on $T_{0}$ should be the temperature at the vertical center of the wind tunnel $(\mathrm{z}=0.455 \mathrm{~m}$ above the plate) at the same streamwise position, where we conduct these temperature measurements. With this, we show corrected results in fig. 4.48(B). These data look more than what was expected. In particular, data with different $\mathrm{Gr}_{x}$ are now rather close to each other, which is expected in this large $\mathrm{Re}_{x}$-regime where buoyancy does not play a big role and hence a dependency of the temperature profile on $\mathrm{Gr}_{x}$ is marginal. 


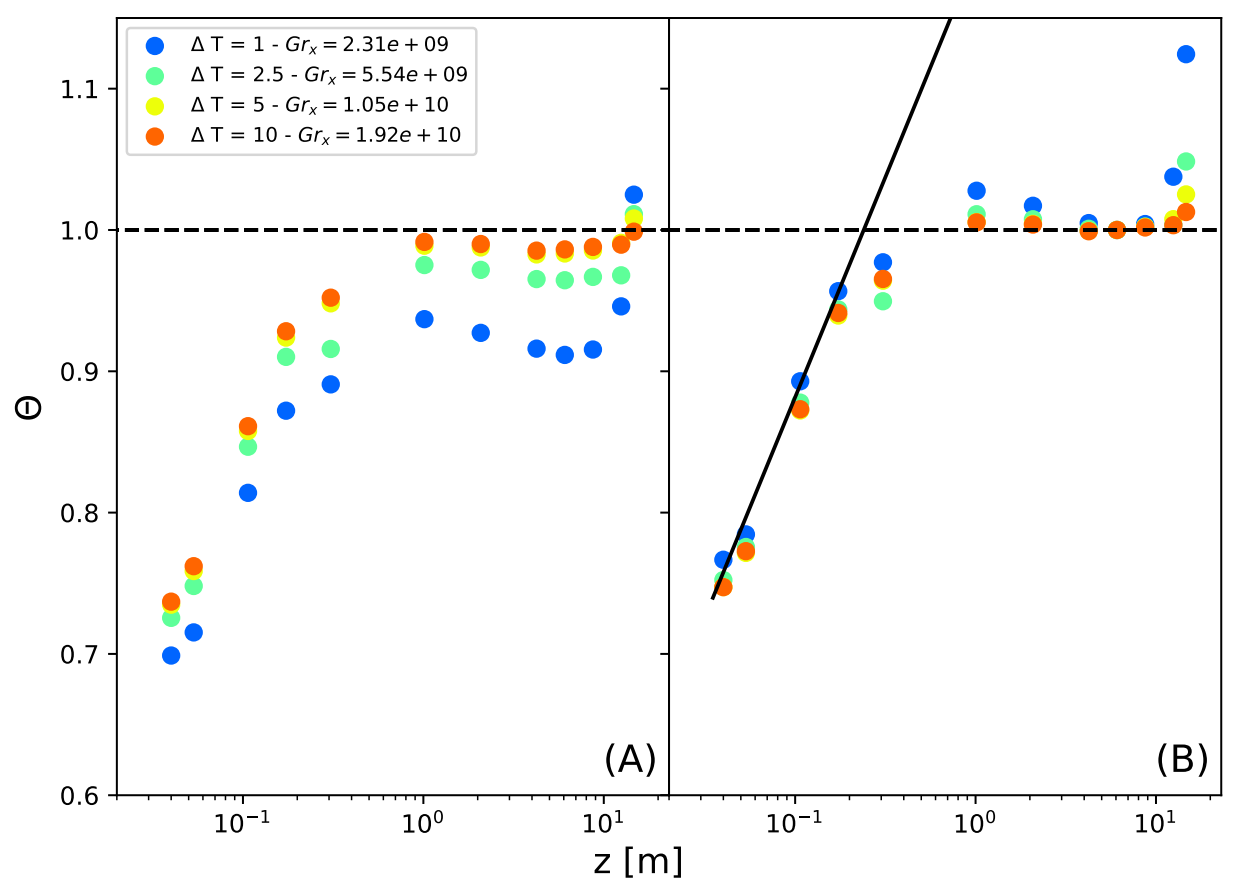

Figure 4.48: Thermal boundary layer with $R e_{x}=1.44 \times 10^{6}-(A)$ Represent $\Theta$ calculated with $T_{0}$ to be the inflow temperature and $(B)$ with $T_{0}$ to be the bulk temperature. The dashed line marks $\Theta=1$. The solid line represent a log-law line for reference. Different symbols mark different $G r_{x}$ (see legend).

We still see a maximum of $\Theta$ at around $\mathrm{z}=7.5 \mathrm{~cm}$ which we cannot really explain. It could be caused by some unwanted large scale circulations inside the wind-tunnel, but we are not sure. The maximum is small and less than $0.1 \mathrm{~K}$ above the base line. It also decreases significantly with increasing $\mathrm{Gr}_{x}$.

We acknowledge that the temperature data acquired here do not have a sufficiently good spatial resolution in order to resolve the distances close to the viscose sub-layer. We in total have also only four points inside the boundary layer and hence we cannot make any statements about the typical temperature profile there. Nevertheless, these points seemingly follow a rather straight line in this semi-logarithmic representation, whose slope with determine to be close to $0.135 \pm 0.009$. This slope represents the average slope over the log-layer and the wake regime in this fully turbulent boundary layer.

In fig. 4.49 we show temperature and velocity profiles for a boundary layer that is in the transitional regime and where the influence of the buoyancy is strong. Velocity data for this case have already been presented in sec. 4.2.2. Here we want to compare the thermal boundary layer with the velocity at the same altitudes.

First we consider fig. $4.49(\mathrm{~A})$, where data for $\mathrm{Re}_{x}=4.7 \times 10^{4}$ are plotted. The 

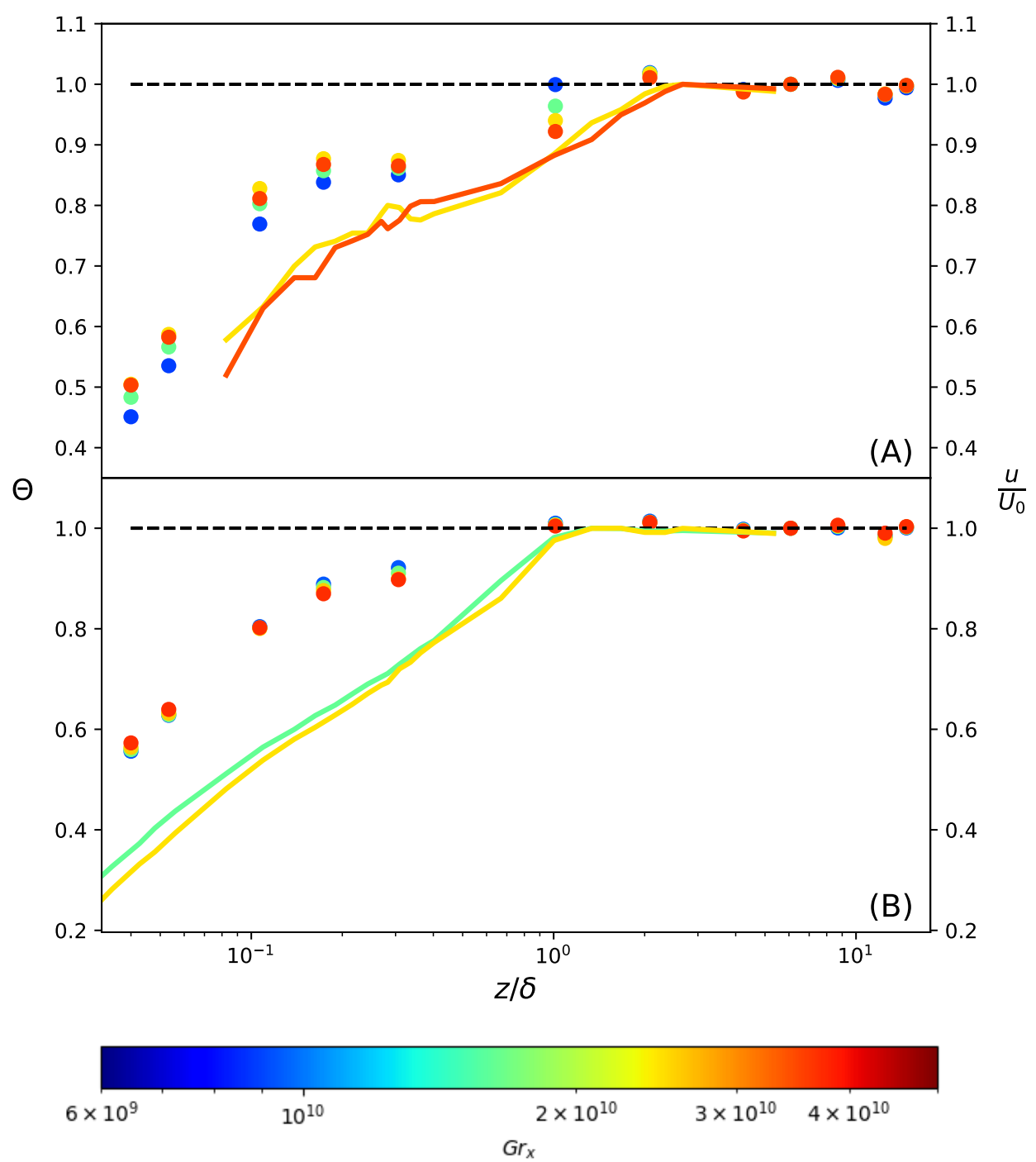

Figure 4.49: Points represent thermal boundary layer $\Theta$ (left y-axis). Lines represents the normalised averaged streamwise velocity $u / U_{0}$ as function of the normalised vertical distance (right $y$-axis). The horizontal dashed line represents $\Theta=1$. (A) Data with $R e_{x}=4.7 \times 10^{4}$. (B) Data with $R e_{x}=1.88 \times 10^{5}$. The different colors of the points and the lines represent $G r_{x}$ according to the color-bar. 
temperature profile does not look strictly monotonic. Again, $\Theta$ increases steeply close to the surface but then seems to reach a plateau at $z / \delta \approx 0.2$, where it settles at $\Theta \approx 0.85$ or so, before it increases towards $\Theta \rightarrow 1$. The plateau only consists of a single point and one is inclined to believe that this is merely an outlier due to a miss-calibrated thermistor for example. However, at a very similar $z$, also the velocity data (solid lines in fig. 4.49A) show a plateau, even though it is less pronounced. Close to the plateau another peculiarity happens. While for smaller $z / \delta<0.3$ points with larger $\mathrm{Gr}_{x}$ (red) are on top of points with smaller $\mathrm{Gr}_{x}$ (blue), the order is reversed and the blue points are on top of the red for larger $z / \delta$. Also, it seems as that the boundary layer height increases with increasing $\mathrm{Gr}_{x}$. While this statement is based on a single point only, it is in good agreement with our findings from the velocity boundary layer analysis in sec. 4.2.2 and general expectations, namely that the boundary layer size increases when momentum transport is enhanced by buoyancy driven convection.

Having this said, we can look at fig. 4.49(B) where the boundary layer is still not fully turbulent, but where the Reynolds number was significantly larger $\left(\operatorname{Re}_{x}=1.88 \times 10^{5}\right)$. Both the velocity and the temperature data look much smoother. However, although weak, the typical feature from above are still clearly visible. These is a small plateau at $z / \delta \approx 0.3$, but also the inversion of the data points for small and larger $\mathrm{Gr}_{x}$. Also here, the blueish points ( $\operatorname{small} \mathrm{Gr}_{x}$ ) are below the red points (large $\mathrm{Gr}_{x}$ ) for small $z / \delta$ but after the plateau, the blueish are clearly above the reddish.

A plateau in temperature suggests a good heat transport in this region. Which might mean that plumes can rise without being broken and mixed with the surrounding fluid. While this is very speculative, it could be that in regions closer to the surface the plumes are ripped easily apart due to the shear stress. For larger $z$ the shear stress smaller and also the turbulence level is still small for the $\operatorname{Re}_{x}$ shown in fig. 4.49 and hence the remaining warm structures (what is left of the initial plumes) can rather freely rise. The plateau then ends at distances $z$, that plumes can reach at a given $x$ because they are also carried away by the mean flow. Warmer plumes at larger $\mathrm{Gr}_{x}$ can reach higher distances and therefore the plateau is larger for the red points then for the blue points in fig. 4.49(A).

In fig. 4.50 we show temperature profiles for three different $\operatorname{Re}_{x}$ for a boundary layer that is in the turbulent regime and where the influence of buoyancy is weak. We also show for $\operatorname{Re}_{x}=7.02 \times 10^{5}$ and $\operatorname{Re}_{x}=9.85 \times 10^{5}$ velocity data taken at similar $\operatorname{Re}_{x}$ that 


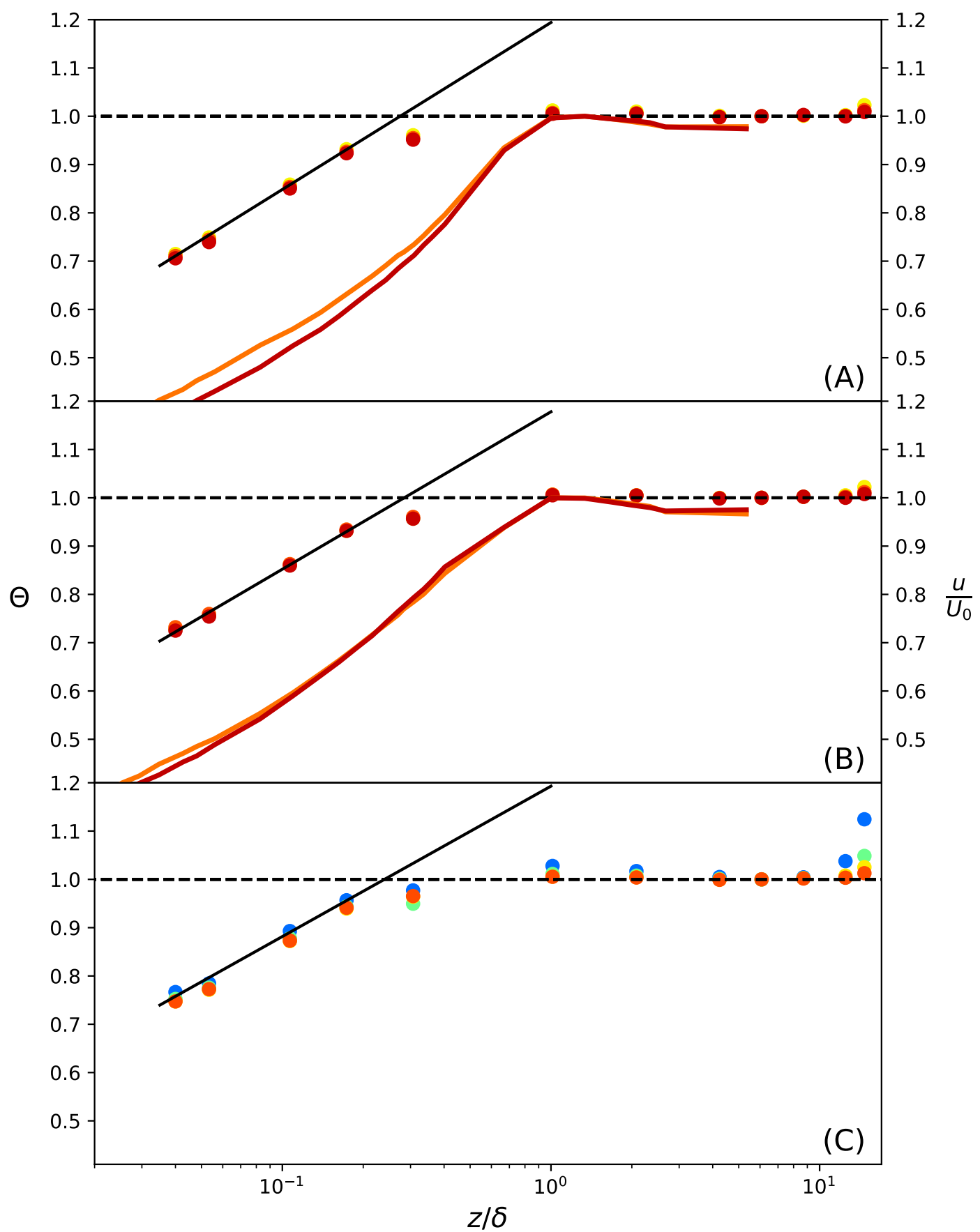

$10^{9}$

$10^{10}$

$G r_{x}$

Figure 4.50: Points represents thermal boundary layer $\Theta$ (left y-axis). Lines represents the normalised averaged streamwise velocity $u / U_{0}$ (right y-axis) as function of the normalised vertical distance. The horizontal dashed line represents $\Theta=1$. The black solid line represent the log scaling. (A) Data with $R e_{x}=7.02 \times 10^{5}$ (B) Data with $R e_{x}=9.85 \times 10^{5}$ (C) Data with $R e_{x}=1.44 \times 10^{6}$. The different colors of the points and the lines represent $G r_{x}$ according to the color-bar. 
have already been presented in sec. 4.2 .

Here we see that in this regime, temperature measurements are rather independent of $\mathrm{Gr}_{x}$. This is expected because $\mathrm{Re}_{x}$ is larger and buoyancy is small in comparison (forced convection). The first four points, $(z / \delta \lesssim 0.2)$ also follow a straight line indicating a logarithmic temperature relationship. Such a logarithmic relationship is predicted as a result of the von Karman defect law (eq. 2.40) and the log-law for the temperature (eq. 4.22) (see also [23]).

If buoyancy does not play a role, the temperature field is advected by the same eddies that also advect the streamwise velocity component. Because the thermal and viscous diffusivities are also very similar $(\operatorname{Pr}=0.7)$, we expect that the temperature and the velocity profile nearly coincide when plotted as in fig. 4.50. However, this is not quite the case. Indeed the logarithmic slopes are very similar for the temperature and velocity profiles, but the temperature $\Theta$ is significantly larger than the velocity $u / U_{0}$. This means that the temperature at a given $z$ is closer to the bulk temperature (colder) as one would have expected, heat has therefore not been transported as efficiently as expected.

We believe that the reason for this discrepancy is again caused by the adiabatic plate in front of the heated plate. At the front of the adiabatic plate $(x=0)$, a velocity boundary layer develops as a result of momentum transport from the plate and advection in streamwise direction. The thermal boundary only starts to develop at $x=1 \mathrm{~m}$ and thus temperature at our measurement point $x=2.35 \mathrm{~m}$ has not been transported so far into the flow and is lower as for the hypothetical where the heating starts at $x=0$.

For a more quantitative analysis, we fit the following function to the data

$$
\Theta(z / \delta)=\beta \ln \left(\frac{z}{\delta}\right)+B
$$

The results of the fits for different $\operatorname{Re}_{x}$ are shown in table 4.7. We see, that $\beta$ slightly increases with increasing $\operatorname{Re}_{x}$. Eq. 4.22 should be compared to an expression derived from the scaling considerations and the the von Karman defect law for the temperature. As presented in [23], one can write

$$
\frac{T(z)-T_{0}}{T_{\tau}}=-\alpha \ln \left(\frac{z}{\delta}\right)+A \quad \text { with } \quad T_{\tau}=\frac{q}{\rho c_{p} u_{\tau}} .
$$


From eq. 4.22 and eq. 4.23, one sees that the coefficients are related as:

$$
\alpha=\beta \frac{\left(T_{w}-T_{0}\right)}{q} \rho c_{p} u_{\tau}
$$

Because $\mathrm{Nu}$ is independent of $\mathrm{Gr}_{x}$ - we neglect buoyancy - the term $\left(T_{w}-T_{0}\right) / q$ is independent of $\mathrm{Gr}_{x}$ for a given $\operatorname{Re}_{x}$. We also know $u_{\tau}$ for two $\mathrm{Re}_{x}$ and can therefore calculate $\alpha$.

\begin{tabular}{|c|c|c|c|}
\hline $\mathrm{Re}_{x} \times 10^{-5}$ & $\beta$ & $B$ & $\alpha$ \\
\hline \hline 7.02 & $0.150 \pm 0.003$ & $1.19 \pm 0.01$ & 1.42 \\
\hline 9.85 & $0.142 \pm 0.005$ & $1.18 \pm 0.01$ & 1.52 \\
\hline 14.4 & $0.135 \pm 0.009$ & $1.19 \pm 0.02$ & $n / a$ \\
\hline
\end{tabular}

Table 4.7: Fit parameters for the fits of eq. 4.22 to the measured temperature.

The corresponding results for $\alpha$ are also listed in tab. 4.7. The value $\alpha$ is often used to define a turbulent Prandtl number $\operatorname{Pr}_{t}=\alpha \kappa$, with $\kappa$ being the von Karman constant. Values for $\alpha$ have been measured in the past for boundary layers on top of a flat plate and are found to be in the range $\alpha=1.80 \ldots 2.10$ [23]. Our values are clearly lower, which we also interpret as an effect of the $1 \mathrm{~m}$ long unheated section at the front of the heated plate. Note also that $\alpha$ are expected to be constant. This is not the case for the two $\operatorname{Re}_{x}$, at which we could determine $\alpha$. There $\alpha$ increases with $\operatorname{Re}_{x}$. Speculative, it is reasonable to assume that the boundary layer is not sufficiently turbulent. Potentially, $\alpha$ increases with increasing $\operatorname{Re}_{x}$ towards the values closer to $\approx 2$. Finding a suitable scaling correction for the adiabatic boundary layer is a task for future modelling. 


\section{Chapter 5}

\section{Conclusion and outlook}

The goal of the research project reported in this thesis was to investigate the dynamics and the properties of a shear boundary layer above a heated plate. We wanted to learn something about the mechanisms that determine the heat flux from the plate in the regimes of forced convection, where heat is advected as a passive scale as well as the regime of mixed convection, where the flow is strongly influenced by buoyancy. The project consisted of two main parts: (i) the design and construction of an experimental setup and (ii) measurements of the velocity, the heat transport, and the temperature profile in the boundary layer above the plate.

In order to test our experimental setup, we have first performed series of measurements with an unheated plate and characterised the velocity boundary layer. For this, we have measured different statistical quantities of the streamwise velocity component at a fixed position $x$ and different distances from the plate. For large $\operatorname{Re}_{x}$ we found typical features of a turbulent boundary layer, such as a velocity profile that is self-similar for different $\operatorname{Re}_{x}$ when plotted in wall units based on the skin friction velocity. For $z$-ranges, where a logarithmic profile of the average streamwise velocity is found, we also observe the $k^{-1}$-scaling for the spectrum of the kinetic energy when $k$ is small and the eddies of the corresponding size are influenced by the presence of the wall. The energy in smaller eddies, on the other hand, scales with $k^{-5 / 3}$ as for isotropic homogeneous turbulence. We quantify the transition between both scaling regimes and found that the transitional wave number scales with the height as $k_{t} \propto z^{-0.41}$ or so.

We also investigated the boundary layer for small Reynolds when the flow was just 
above $\mathrm{Re}_{\text {ind }}$ at which a laminar boundary layer becomes unstable, i.e., the transitional regime. We have used the M-TERA method to quantify the intermittency of the flow when it transits from almost laminar to fully turbulent with increasing $\operatorname{Re}_{x}$.

Heat provided from the bottom clearly changed the velocity profile even for the largest $\mathrm{Re}_{x}$, where buoyancy was expected not to play an important role. In particular, with increasing heat (increasing $\mathrm{Gr}_{x}$ ) both the average velocity and its variance decreased in the buffer layer when the skin friction velocity $u_{\tau}$ is taken as the same as for the unheated case. In fact, this observation shares some similarities to observations made over rough surfaces. We interpret these observations such that the introduction of heat destabilises the lower part of the boundary layer and hence increases the contribution of advection for momentum transport.

In the quasi-turbulent boundary layer regime, when the flow is slow (small $\operatorname{Re}_{x}$ ), we have observed that the introduction of heat affects the boundary layer much stronger because now the buoyancy is large compared to the advective forces. As a result, the quasi-turbulent boundary layer becomes more turbulent resulting in an increased turbulent intermittency. Since also the momentum transport in the wall-normal direction is increased, the boundary layer size increases as well with increasing $\mathrm{Gr}_{x}$.

In our measurements of the heat transfer from the plate, we found a clear distinction between the free and forced convection regime. With forced convection, we observed a power-law relation between the heat transfer and the shear stress as $N u_{x} \propto R e_{x}^{0.8}$, independent of $\mathrm{Gr}_{x}$. On the other side, in the regime of nearly free convection, we rather observed a stronger correlation between the heat transfer and the surface temperature as $N u_{x} \propto G r_{x}^{0.205}$. In this regard we have proposed a new length scale $\delta$ for the Grashof number so that when $N u_{N}=N u_{x} / G r_{\delta}$ is plotted against $R i_{\delta}=G r_{\delta} / R e_{x}^{2}$, data for different Re and Gr collapse. Data plotted in this way exhibit two clearly distinct regimes with two different power-laws, i.e., $N u_{N} \propto R i_{\delta}^{-0.315}$ in forced convection regime and $N u_{N} \propto R i_{\delta}^{-0.098}$ in the free convection one.

Although measurements of the vertical temperature profile have been conducted somehow in a hurry, the results are surprisingly satisfying. For fast flows (large $\operatorname{Re}_{x}$ ), we observed the expected logarithmic scaling of the temperature inside the boundary layer. However, the temperature was much lower than what has been found by others (see [23]). We explain this discrepancy with the adiabatic plate in front of the heating section and 
hence with a thermal boundary layer that starts to develop later and therefore is smaller than the velocity boundary layer. For slow flows $\left(\operatorname{small} \mathrm{Re}_{x}\right)$ the thermal boundary layer is somehow irregular and even suggests to be stratified at some altitudes. While the spatial resolution was too small to draw a clear picture, this observation is very much in accordance with the velocity profile under the same condition. We see in the temperature measurements also a growth of the boundary layer with increasing $\mathrm{Gr}_{x}$, as was already suggested by the velocity measurements.

In this work, we have achieved our primary goals of collecting data on the heat transfer, velocity and thermal boundary layer for the free, mixed and forced convection regime, at Reynolds numbers ranging from just above its critical value where the laminar boundary layer becomes unstable up to values for fully developed turbulence. These results helped us to achieve a general better understanding of the dynamics induced by the introduction of heat from below inside the boundary layer. However, we have also encountered various experimental problems and have gained ideas for improvements of the apparatus as well as for further measurements. Since our experiment has been designed to be modular and flexible, we believe such measurements can be done rather easily.

For example, the wind tunnel does not account for the growth of boundary layers at the inside of the wind tunnel. This growth should be detected by monitoring the pressure distribution along the flow. So far we have measured the velocity at only one streamwise position $x$, even though the boundary layer grows in $x$-direction. Conducting velocity measurements for different streamwise locations along the entire plate is certainly very important for understanding the evolution of the boundary layer height as well as to investigate thermal plumes that are carried along with the flow. This would benefit also the analysis of the streamwise evolution of the turbulence intermittency $\gamma$ in the quasi-laminar regime, where more experiments are required to validate our observations.

The scaling of the turbulent kinetic energy with wave number and the change from the $k^{-1}$ to the $k^{-5 / 3}$ regime are certainly worth being studied in more depth because from these scalings one learns something about the energy transfer from large to small scales and how this is affected by the presence of a solid wall.

When we constructed the experiment, we installed a $1 \mathrm{~m}$ long adiabatic plate in front of the heated section. Our initial goal was to have a fully developed boundary layer which is then perturbed by buoyancy. However, as it turns out, developing scaling arguments for 
this system is difficult. There are no good models that allow corrections for the adiabatic front plate. In a refined experiment, we probably would remove the adiabatic front plate, which would not only help to compare our measurements with existing models, but we could compare results with and without the front plate and possibly better understand its influence. The ability to easily and cheaply swap the front surface allows for any kind of design, that can also include favourable and adverse pressure gradient setups.

Another interesting question would be, how surface texture influences the state of the boundary layer and the heat transport from it. This holds not only for the adiabatic front but also for the currently heatable section.

The $2 \mathrm{~m}^{2}$ surface allows to host any kind of topological profile, including water surfaces. For example one could model a coastal area and, once heated from below, study how the velocity field evolves in these regions under different conditions. In such applications, one could use smoke to clearly visualize the flow.

Although hot-wire anemometry has the advantage that it allows for very fast measurements, its disadvantage is that one can only measure a single velocity component at a single point in space. For the future we suggest using optical measurement techniques such as particle image velocimetry - PIV, laser doppler velocimetry - LDA or lagrangian particle tracking - LPT. One of the main benefits of such techniques is the ability to measure a larger region of the fluid simultaneously rather than a single point at a time. Also, with suitable particles, the measurements are less invasive than the hot-wire measurements, which are currently installed on an extended arm. This together with the ability to measure velocity in the sub-mm distance from the surface (for LDA) and maintaining a comparable space resolution to the one of the hot-wire would result in a more comprehensive picture of the velocity field.

For these methods, the flow needs to be seeded with small neutrally buoyant particles. Unfortunately, this cannot be easily done in our open wind tunnel, as the air and thus the particles are blown directly into the experimental hall, which in the best case causes pollution of the experimental hall, but in the worst case poses a severe health risk. Therefore, in order to use optical measurement methods, one has to first install an appropriate filter at the outlet of the wind tunnel which filters the particle out but creates a sufficiently small resistance of the flow. In light of the ongoing Covid-19 pandemic, our department is currently conducting extensive testing of different materials that are suitable for efficient 
aerosol filters. We are convinced that the same material can be used in the wind tunnel as well.

Altogether, the modular nature of the setup leaves space for an extremely high number of different applications and we hope this experimental setup will be used to produce a lot of valuable research data in the future. In short, we believe our experimental apparatus has proven its potential so far and we believe its productive life has just begun. 


\section{Bibliography}

[1] J.M. Burgers. The motion of a fluid in the boundary layer along a plain smooth surface. Proceedings of the First International Congress for Applied Mechanics, Delft,. [33, 162, 420, 440], pages 113-128, 1924.

[2] B.G. Van der Hegge Zijnen. Measurements of the velocity distribution in the boundary layer along a plane surface. PhD thesis, TU Delft, 1924.

[3] M. Hansen. Die Geschwindigkeitsverteilung in der Grenzschicht an einer eingetauchten Platte. ZAMM. Z. angew. Math. Mech., 8:185-199, 1928. doi: 10.1002/ zamm.19280080303.

[4] H.L. Dryden. Turbulence and the boundary layer. J. of the Aeronautical Sciences, 6:85-100 and 101-105, 1939. doi: 10.2514/8.777.

[5] O. Reynolds. An experimental investigation of the circumstances which determine whether the motion of water shall be direct or sinuous, and of the law of resistance in parallel channels. Philosophical Transactions of the Royal Society of London, 174: 935-982, 1883. URL http://www.jstor.org/stable/109431.

[6] G.I. Taylor. The spectrum of turbulence. Proc. R. Soc., 164, 1938. doi: 10.1098/ rspa. 1938.0032.

[7] A. N. Kolmogorov. The local structure of turbulence in incompressible viscous fluid for very large reynolds numbers. Proc. R. Soc. Lond. A, 434:9-13, 1941. doi: 10.1098/rspa.1991.0075.

[8] G.I. Taylor. Statistical theory of turbulence. Proc. R. Soc. A., 151:421-444, 1935. doi: 10.1098/rspa.1935.0158. 
[9] G.I. Taylor. Statistical theory of turbulence-ii. Proc. R. Soc. A, 151:444-454, 1935. doi: 10.1098/rspa.1935.0159.

[10] G.I. Taylor. Statistical theory of turbulence iii-distribution of dissipation of energy in a pipe over its cross-section. Proc. R. Soc. A, 151:455-464, 1935. doi: 10.1098/ rspa.1935.0160.

[11] G.I. Taylor. Statistical theory of turbulence iv-diffusion in a turbulent air stream. Proc. R. Soc. A, 151:465-478, 1935. doi: 10.1098/rspa.1935.0161.

[12] G.I. Taylor. Statistical theory of turbulence v-effect of turbulence on boundary layer theoretical discussion of relationship between scale of turbulence and critical resistance of spheres. Proc. R. Soc. Lond. A, 156:307-317, 1935. doi: /10.1098/ rspa.1936.0149.

[13] L.F. Richardson. Weather Prediction by Numerical Process. Cambridge University Press, 1922.

[14] Pablo Ouro. Large-Eddy Simulation of Tidal Turbines. PhD thesis, Cardiff University, 2017. URL http://orca.cf.ac.uk/103301/.

[15] L. Onsager. The distribution of energy in turbulence. Phys. Rev., 68:281, 1945.

[16] L. Prandtl. Über Flüssigkeitsbewegung bei sehr kleiner Reibung (Motion of fluids with very little viscosity). Verhandlungen des 3 Internationalen MathematikerKongresses Heidelberg., page 484-491, 1904.

[17] H. Blasius. Grenzschichten in Flüssigkeiten mit kleiner Reibung. Z. Math. Physik, $56: 1-37,1908$.

[18] Th. von Karman. Mechanical similitude and turbulence. Tech. Mem. NACA, no. 611, 1931 (or Nachrichten von der Gesellschaft der Wissenschaften zu Göttingen, Fachgruppe 1 (Mathematik), 5: 58-76), 1930. URL https://ntrs.nasa.gov/citations/ 19930094805.

[19] J. Nikuradse. Strömungsgesetze in rauhen Rohren. VDI-Forschungsheft, 361:1, 1933. 
[20] A.A. Izakson. On the formula for the velocity distribution near walls. Techn. Phys. USSR, 4:155-162, 1937.

[21] C. B. Millikan. A critical discussion of turbulent flow in channels and circular tubes. In Proc. 5th Int. Congress on Applied Mechanics, pages 386-392, 1939.

[22] R. Mises. Some remarks on the laws of turbulent motion in tubes. Calif. Inst. Techn. Press. Pasadena., Th. V. Karman Anniversary Volume:317-327, 1941.

[23] B.A. Kader, A.M. Yaglom. Heat and mass transfer laws for fully turbulent wall flows. Intnernational Journal of Heat and Mass Transfer, 15:2329-2351, 1972. doi: 10.1016/0017-9310(72)90131-7.

[24] F.M. White. A new integral method for analyzing the turbulent boundary layer with arbitrary pressure gradient. ASME. J. Basic Eng., 91:371-376, 1969. doi: 10.1115/1.3571122.

[25] J.M. Österlund. Experimental studies of zero pressure-gradient turbulent boundarylayer flow. Ph.D. thesis, Department of Mechanics, Royal Institute of Technology, Stockholm, 1999, 1999.

[26] A. Kendall, M. Koochesfahani. A method for estimating wall friction in turbulent boundary layers. Collection of Technical Papers - 25th AIAA Aerodynamic Measurement Technology and Ground Testing Conference, 2, 06 2006. doi: $10.2514 / 6.2006-3834$.

[27] D.B. Spalding. A single formula for the "law of the wall". J. Appl. Mech., 28: 455-458, 1961. doi: 10.1115/1.3641728.

[28] A.J. Musker. Explicit expression for the smooth wall velocity distribution in a turbulent boundary layer. AIAA J., 17:655-657, 1979. doi: 10.2514/3.61193.

[29] H. Schlichting, K. Gersten. Boundary-Layer Theory - "7th Edition. Springer-Verlag Berlin Heidelberg, 1979.

[30] K. Gersten, H. Herwig. Strömungsmechanik. Grundlagen der Impuls-, Wärme- und Stoffübertragung aus asymptotische. Springer, 1992. 
[31] A.E. Perry, S. Henbest, M.S. Chong. A theoretical and experimental study of wall turbulence. J. Fluid Mech., 165:163-199, 1986. doi: 10.1017/S002211208600304X.

[32] P.A. Davidson. Turbulence an introduction for Scientists and Engineers. Oxford University Press, 2004.

[33] N. Hutchins, K. Chauhan, I. Marusic, J. Monty, J. Klewicki et al. Towards Reconciling the Large-Scale Structure of Turbulent Boundary Layers in the Atmosphere and Laboratory. Towards reconciling the large-scale structure of turbulent boundary layers in the atmosphere and laboratory. Boundary-Layer Meteorol., 145:273-306, 2012. doi: 10.1007/s10546-012-9735-4.

[34] N. Reina, L. Mahrt. A study of intermittent turbulence with cases-99 tower measurments. Boundary-Layer Meteorology, 114:367-387, 2005. doi: 10.1007/s10546004-0857-1.

[35] M. Guala, S. Hommema, R.J. Adrian. Large-scale and very-large-scale motions in turbulent pipe flow. Journal of Fluid Mechanics, 554:521-542, 2006. doi: 10.1017/ S0022112006008871.

[36] J. Zhou, R.J. Adrian, S. Balachandar, T.M. Kendall. Mechanisms for generating coherent packets of hairpin vortices in channel flow. Journal of Fluid Mechanics, 387:353-396, 1999. doi: 10.1017/S002211209900467X.

[37] B.J. Balakumar, R.J. Adrian. Large- and very-large-scale motions in channel and boundary-layer flows. Phil. Trans. R. Soc. A., 365:665-681, 2007. doi: 10.1098/ rsta.2006.1940.

[38] N. Hutchins, I. Marusic. Evidence of very long meandering features in the logarithmic region of turbulent boundary layers. Journal of Fluid Mechanics, 579:1-28, 2007. doi: 10.1017/S0022112006003946.

[39] J. Monty, R. Williams, M. Chong. Large-scale features in turbulent pipe and channel flows. Journal of Fluid Mechanics, 589:147-156, 2007. doi: 10.1017/ S002211200700777X. 
[40] X. Zheng G. Wang. Very large scale motions in the atmospheric surface layer: A field investigation. Journal of Fluid Mechanics, 802:464-489, 2016. doi: 10.1017/ jfm.2016.439.

[41] S. Baley, A. Smits. Experimental investigation of the structure of large- and verylarge-scale motions in turbulent pipe flow. Journal of Fluid Mechanics, 651:339-356, 2010. doi: 10.1017/S0022112009993983.

[42] R. Mathis, J.P. Monty, N. Hutchins, I. Marusic. Comparison of large-scale amplitude modulation in turbulent boundary layers, pipes, and channel flows. Physics of Fluids, 21:111703, 2009. doi: 10.1063/1.3267726.

[43] F. Eich, C.J. Kaehler. Scaling and dynamics of large-scale structures in turbulent boundary layers with pressure gradients. In 10th International Symposium on Turbulence and Shear Flow Phenomena (TSFP10), Chicago, USA, July, 2017.

[44] H.Z. Liu, F. Hu, Z.X. Hong Q. Li. Characteristic of the urban boundary layer under strong wind condition in beijing city. J. Grad School Chin Acad Sci., 21:40-44, 2004.

[45] M.K. Lauren, M. Menabde, G.L. Austin. Analysis and simulation of surface-layer winds using multiplicative cascade models with self-similar probability densities. Boundary-Layer Meteorology, 100, 2001. doi: 10.1023/A:1019210914169.

[46] X. Yumao, P. Yinong, S. Aidong. Spectral characteristics and multi-scale structure of the boundary-layer wind field during cold front passages over east china. Boundary-Layer Meteorology, 85:423-446, 1997. doi: 10.1023/A:1000599518302.

[47] Kerstin Avila, David Moxey, Alberto de Lozar, Marc Avila, Dwight Barkley, and Björn Hof. The onset of turbulence in pipe flow. Science, 333(6039):192-196, 2011. ISSN 0036-8075. doi: 10.1126/science.1203223. URL http://science.sciencemag. org/content/333/6039/192.

[48] H. Schlichting, K. Gersten. Boundary-Layer Theory - 9th Edition. Springer-Verlag Berlin Heidelberg, 2017. 
[49] G.B. Schubauer, P.S. Klebanoff. Contributions on the mechanics of boundarylayer transition. Technical report, 1955. URL https://ntrs.nasa.gov/citations/ 19930092285.

[50] H.W. Emmons. The laminar-turbulent transition in a boundary layer-part i. Journal of the Aeronautical Sciences, 18(7):490-498, 1951. doi: 10.2514/8.2010.

[51] G.B. Schubauer, H.K. Skramstad. Laminar boundary-layer oscillations and stability of laminar flow. Journal of the aereonautical sciences, 14:69, 1947. doi: 10.2514/8. 1267.

[52] A.M. Obukhov. Turbulence in a thermally inhomogeneous medium. Trudy Inst. Teor. GeojYz.. Akad. Nauk SSSR, 1:95-115, 1946.

[53] H.B. Squire. The friction temperature: A useful parameter in heat-transfer analysis. Proc. General Discus. Heat Transfer Inst. Mech. Engng. and ASME. London, pages 185-186, 1951.

[54] A.S. Monin, and A.M. Obukhov. Basic laws of turbulent mixing in the surface layer of the atmosphere. e. Trudy Geofiz Inst Acad Nauk SSSR, 24:163-187, 1954.

[55] A.M. Obukhov. Turbulence in an atmosphere with a non-uniform temperature. Boundary-Layer Meteorol., 2:7-29, 1971.

[56] E. Bodenschatz, M. Eckert. prandtl and the göttingen school.

[57] G. Ahlers, D. Funfschilling, and E. Bodenschatz. Transitions in heat transport by turbulent convection at Rayleigh numbers up to $10^{15}$. New J. Phys., 11:123001-1-18, 2009 .

[58] Dantec. Hot-wire spec description, 2020. https://www.dantecdynamics.com/ components/hot-wire-and-hot-film-probes/single-sensor-probes/miniature-wire/.

[59] Dantec. Hot-wire product page, 2020. https://www.dantecdynamics.com/product/ miniature-wire-probe-boundary-layer/.

[60] Dantec. Measurement Principles of CTA, 2020. https://www.dantecdynamics. com/solutions-applications/solutions/fluid-mechanics/constant-temperatureanemometry-cta/measurement-principles-of-cta/. 
[61] Testo. Testo $405 i$ anemometer technical data, 2020. https://www.testo.com/enUS/testo-405i/p/0560-1405.

[62] L.V. King. On the convection of heat from small cylinders in a stream of fluid: Determination of the convection constants of small platinum wires with applications to hot-wire anemometry. Philosophical Transactions of the Royal Society of London. Mathematical or Physical Character, 214:373-432, 1914. doi: 10.1098/rsta.1914. 0023.

[63] L.V. King. On the precision measurement of air velocity by means of the linear hot-wire anemometer. The London, Edinburgh, and Dublin Philosophical Magazine and Journal of Science, 29:556-577, 1915. doi: 10.1080/14786440408635334.

[64] L.V. King, D.Sc. McGill. The linear hot-wire anemometer and its applications in technical physics. Journal of the Franklin Institute, 181:1-25, 1916. doi: 10.1016/ S0016-0032(16)90107-8.

[65] D. Coles. The law of the wake in the turbulent boundary layer. Journal of Fluid Mechanics, 1:191-226, 1956. doi: 10.1017/S0022112056000135.

[66] S.Kokoska and D. Zwillinger. Standard probability and Statistics tables and formulae. Chapman \& Hall/CRC, 1999. doi: 10.1201/9781420050264.

[67] JAVIER JIMÉNEZ. Turbulent velocity fluctuations need not be gaussian. Journal of Fluid Mechanics, 376:139-147, 1998. doi: 10.1017/S0022112098002432.

[68] Richard J. A. M. Stevens, Michael Wilczek, and Charles Meneveau. Large-eddy simulation study of the logarithmic law for second- and higher-order moments in turbulent wall-bounded flow. Journal of Fluid Mechanics, 757:888-907, 2014. doi: 10.1017/jfm.2014.510.

[69] F. Durst, J. Jovanovic and L. Kanevce. Probability density distribution in turbulent wall boundary-layer flows. Turbulent Shear Flows, 5:197-220, 1987. doi: 10.1007/ 978-3-642-71435-1_18.

[70] C.M. Tchen. On the spectrum of energy in turbulent shear flow. Journal of Research 
of the National Bureau of Standards, 50:51-62, 1953. URL https://nvlpubs.nist.gov/ nistpubs/jres/50/jresv50n1p51_A1b.pdf.

[71] C.M. Tchen. Transport processes as foundations of the Heisenberg and Obukhoff theories of turbulence. Phys. Rev., 93:4, 1954. doi: 10.1103/PhysRev.93.4.

[72] I. Marusic, B.J. McKeon, P.A. Monkewitz, H.M. Nagib, A.J. Smits, and K.R. Sreenivasan. Wall-bounded turbulent flows at high Reynolds numbers: Recent advances and key issues. Physics of Fluids, 22:065103, 2010. doi: 10.1063/1.3453711.

[73] J.C.R. Hunt, and J.F. Morrison. Eddy structure in turbulent boundary layers. European Journal of Mechanics - B/Fluids, 19:673-694, 2000. doi: 10.1016/S09977546(00)00129-1.

[74] T.B. Nickels, I. Marusic, S. Hafez, M. S. Chong. Evidence of the $k^{-1}$ law in a highReynolds-number turbulent boundary layer. Phys. Rev. Lett., 95:074501, 2005. doi: 10.1103/PhysRevLett.95.074501.

[75] B. Eckhardt. Transition to turbulence in shear flows. Physica A: Statistical Mechanics and its Applications, 504:121-129, 2018. doi: 10.1016/j.physa.2018.01.032.

[76] D.H. Zhang, Y.T. Chew, S.H. Winoto. A proposed intermittency measurement method for transitional boundary layer flows. Experiments in Fluids, 19:426-428, 1995. doi: 10.1007/BF00190260.

[77] D.H. Zhang, Y.T. Chew, S.H. Winoto. Investigation of intermittency measurement methods for transitional boundary layer flows. Exp. Therm. and Flu. Sci., 12:433443, 1996. doi: 10.1016/0894-1777(95)00133-6.

[78] J. Jimenez. Turbulent flows over rough walls. Annual Review of Fluid Mechanics, 36:173-196, 2004. doi: 10.1146/annurev.fluid.36.050802.122103.

[79] M.P. Schultz, and K.A. Flack. The rough-wall turbulent boundary layer from the hydraulically smooth to the fully rough regime. Journal of Fluid Mechanics, 580: 381-405, 2007. doi: 10.1017/S0022112007005502. 
[80] K.A. Flack, M.P. Schultz, and T.A. Shapiro. Experimental support for Townsend's Reynolds number similarity hypothesis on rough walls. Physics of Fluids, 17:035102, 2005. doi: 10.1063/1.1843135.

[81] D.T. Squire, C. Morrill-Winter, N. Hutchins, M.P. Schultz, J.C. Klewicki, and I. Marusic. Comparison of turbulent boundary layers over smooth and rough surfaces up to high Reynolds numbers. Journal of Fluid Mechanics, 795:210-240, 2016. doi: $10.1017 / \mathrm{jfm} .2016 .196$.

[82] P.R. Spalart, J.H. Watmuff. Experimental and numerical study of a turbulent boundary layer with pressure gradients. Journal of Fluid Mechanics, 249:337-371, 1992. doi: 10.1017/S002211209300120X.

[83] J.P. Monty, Z. Harun, and I. Marusic. A parametric study of adverse pressure gradient turbulent boundary layers. International Journal of Heat and Fluid Flow, 32:575-585, 2011. doi: 10.1016/j.ijheatfluidflow.2011.03.004.

[84] Video smoke-seeded with $R e_{x}=4.7 \times 10^{4}$ and $G r_{x}=0$. http://www.lfpn.ds.mpg. de/convection/download/40cms_0Dt.mp4, .

[85] Video smoke-seeded with $R e_{x}=4.7 \times 10^{4}$ and $G r_{x}=1.04 \times 10^{10}$. http://www. lfpn.ds.mpg.de/convection/download/40cms_5Dt.mp4, .

[86] Video smoke-seeded with $R e_{x}=4.7 \times 10^{4}$ and $G r_{x}=6.26 \times 10^{10}$. http://www.lfpn. ds.mpg.de/convection/download/40cms_30Dt.mp4, .

[87] Video smoke-seeded with $R e_{x}=4.7 \times 10^{4}$ and $G r_{x}=2.09 \times 10^{9}$. http://www.lfpn. ds.mpg.de/convection/download/40cms_1Dt.mp4, .

[88] Video smoke-seeded with $R e_{x}=4.7 \times 10^{4}$ and $G r_{x}=2.08 \times 10^{10}$. http://www.lfpn. ds.mpg.de/convection/download/40cms_10Dt.mp4, .

[89] E. Rodríguez-López1, P.J.K. Bruce, and O.R.H. Buxton. A robust post-processing method to determine skin friction in turbulent boundary layers from the velocity profile. Exp. Fluids, 56:68, 2015. doi: 10.1007/s00348-015-1935-5. 
[90] John H. Lienhard V. Heat transfer in flat-plate boundary layers: A correlation for laminar, transitional, and turbulent flow. J. Heat Transfer., 142:061805, 2020. doi: 10.1115/1.4046795.

[91] S. Sugawara, T. Sato, and S. Yoshimura. Experimental researches on heat transfer on the surface of flat plate in forced flow (1st report). Trans. Jpn. Soc. Mech. Eng., 17:122-129, 1951. doi: 10.1299/kikai1938.17.62_122.

[92] W.M. Kays, S.J. Kline W.C. Reynolds. Heat transfer in the turbulent incompressible boundary layer 1 - constant wall temperature. NASA-MEMO-12-1-58W/PT1, 1958. URL https://ntrs.nasa.gov/citations/19980228020.

[93] W.C. Reynolds, W.M. Kays, S.J. Kline. Heat transfer in the turbulent incompressible boundary layer 2 - step wall temperature distribution. NASA-MEMO-12-258W/PT2, 1958. URL https://ntrs.nasa.gov/citations/19980228022.

[94] W.C. Reynolds, W.M. Kays, S.J. Kline. Heat transfer in the turbulent incompressible boundary layer 3 - arbitrary temperature and heat flux. NASA-MEMO-12-358W, 1958. URL https://ntrs.nasa.gov/citations/19980228051.

[95] W.C. Reynolds, W.M. Kays, S.J. Kline. Heat transfer in the turbulent incompressible boundary layer 4 - effect of location of transition and prediction of heat transfer in a known transition region. NASA-MEMO-12-4-58W, 1958. URL https://ntrs.nasa.gov/citations/20050158659.

[96] X.A. Wang. An experimental study of mixed, forced, and free convection heat transfer from a horizontal flat plate to air. J. Heat Transfer., 104:139-144, 1982. doi: $10.1115 / 1.3245040$.

[97] R.J.Goldstein, and Kei-Shun Lau. Laminar natural convection from a horizontal plate and the influence of plate-edge extensions. Journal of Fluid Mechanics, 129: 55-75, 1983. doi: 10.1017/S0022112083000646.

[98] E.M. Sparrow, R. Eichhorn, J.L. Gregg. Combined forced and free convection in a boundary layer flow. The Physics of Fluids 2, 319, 1959. doi: 10.1063/1.1705928. 
[99] T.S. Chen, E.M. Sparrow, A.Mucoglu. Mixed convection in boundary layer flow on a horizontal plate. J. Heat Transfer., 99:66-71, 1977. doi: 10.1115/1.3450657.

[100] G.E. Robertson, J.H. Seinfeld, L.G. Leal. Combined forced and free convection flow past a horizontal flat plate. AIChE Journal, 19:998-1008, 1973. doi: 10.1002/aic. 690190517.

[101] C.A. Hieber. Mixed convection above a heated horizontal surface. International Journal of Heat and Mass Transfer, 16:769-785, 1973. doi: /10.1016/0017-9310(73) 90090-2. 


\section{Acknowledgments}

First and foremost, I would like to thank my advisor, Dr. Stephan Weiss. His support has been priceless during these years. He has always found time to respond to my questions and listen to my ideas. His ability to face the new coming problems, steer the project in the right directions and his positive attitude created in me great respect in him as a scientist, as a leader, and above all has a Human. I thank also my thesis committee members - Prof. Andreas Tilgner and Dr. Olga Shishkina for their help and support. I thank Prof. Eberhard Bodenschatz, who gave me a home in his department and covered all the expenses for this experiment.

I thank my family that supported my studies and always believed in me, a piece of luck that is too often underestimated. Paolo Lia, Cristina Cosenza and of course Dr. Antonio Anastasi that lefted us too early, but will never be forgotten and will always be with me. I thank Dr. Prashant Prabhakaran, Augustinus Bertens, Marcel Wedi, Hiu Fai Yik, Marcel Schroeder, Dr. Lukas Zwirner, Dr. Xuan Zhang, Philipp Reiter for the countless our spent talking about science and/or for the support offered during the writing of the thesis proofreading and dedicating their precious time to help. There is also an endless amount of amazing peoples that I meet during these years that I would love to thanks for the time spent together: Dr. Laura Turco, Dr. Johannes Güttler and Jasmin Spieß, Antonio Landeta, Dr. Gerrit Green, Aina Gallemí-Pérez, Torben Neumann, Dr. Soda Liu, and everyone that I had the luck to converse within our institute. I would like to acknowledge and thanks to Dr. Artur Kubitzek for the help in the design of the experimental setup, Andreas Renner for the help in the build of the experimental setup, Andreas Kopp for his disponibility to help whenever needed. I would also like to thank all the people from our Mechanical and Electrical workshop for their help and support. I thank Dr. Freja Nordsiek, Dr. Gholamhossein Bagheri, and Dr. Christian Kuchler, for offering their knowledge and expertise, and be always there to respond to questions and ready to offer suggestions. I would also like to thanks all the personnel of our institute that has always been helpful and supportive, with a special mention to Angela Gremmel, Caroline Siebert and Frauke Bergmann (PBCS/GGNB) for her help over the years.

Last but not least, I acknowledge financial support by the Deutsche Forschungsgemeinschaft (DFG) via the Priority Programme SPP - Turbulent Superstructures for my salary and the useful annual meetings, without which this experiment would have not been possible. I also acknowledge the IMPRS travel grant over the years for attending various conferences. 